\title{
AUTOMATED CLASSIFICATION AND ENHANCED CHARACTERIZATION OF CIRCULATING TUMOR CELLS BY IMAGE CYTOMETRY
}

Tycho Scholtens 


\section{Automated Classification AND ENHANCED CHARACTERIZATION OF CIRCULATING TUMOR CELLS BY IMAGE CYTOMETRY}

Tycho Marinus Scholtens 
Samenstelling promotiecommissie:

Prof. dr. G. van der Steenhoven

Prof. dr. L.W.M.M. Terstappen MD

Prof. dr. J.L. Herek

Prof. dr. J.C.T. Eijkel

Prof. dr. H.J.M. Groen

Prof. dr. A.G.J.M. van Leeuwen

Dr. C. Rao
Universiteit Twente (voorzitter en secretaris)

Universiteit Twente (promotor)

Universiteit Twente

Universiteit Twente

UMC Groningen

AMC Amsterdam

Veridex LLC

This work was financially supported by Veridex LLC.

Copyright (c) 2012 by Tycho Scholtens, Enschede, The Netherlands.

All rights reserved. No part of this book may be reproduced or transmitted, in any form or by any means, electronic or mechanical, including photocopying, microfilming, and recording, or by any information storage or retreival system, without prior written permission of the author.

Typeset with $\mathrm{LT}_{\mathrm{E}} \mathrm{X}$.

ISBN 978-90-365-3421-5

DOI $\quad 10.3990 / 1.9789036534215$ 


\title{
AUTOMATED CLASSIFICATION AND ENHANCED CHARACTERIZATION OF CIRCULATING TUMOR CELLS BY IMAGE CYTOMETRY
}

\author{
PROEFSCHRIFT
}

ter verkrijging van

de graad van doctor aan de Universiteit Twente, op gezag van de rector magnificus, prof. dr. H. Brinksma,

volgens besluit van het College voor Promoties in het openbaar te verdedigen

op woensdag 3 oktober 2012 om 16.45 uur

door

Tycho Marinus Scholtens

geboren op 26 september 1978

te Oldenzaal 
Dit proefschrift is goedgekeurd door:

Prof. dr. L.W.M.M. Terstappen MD (promotor) 


\section{CONTENTS}

1 Introduction $\quad 1$

1.1 Introduction to cancer . . . . . . . . . . . . . . . 1

1.2 The use of circulating tumor cells to improve treatment decisions in the clinic . . . . . . . . . . . . 3

1.3 Molecular therapy targets related to CTC . . . . . . . . 5

1.4 CTC detection technologies . . . . . . . . . . . 6

1.5 The CellSearch system . . . . . . . . . . . . . 8

1.5.1 CellTracks AutoPrep . . . . . . . . . . . . 8

1.5.2 CellTracks Magnest . . . . . . . . . . . . . . . 9

1.5.3 CellTracks Analyzer II . . . . . . . . . . . . . . . . . 9

1.6 Challenges addressed in this thesis . . . . . . . . . . . . 12

1.7 Thesis outline . . . . . . . . . . . . . . . . . . 14

1.8 References . . . . . . . . . . . . . . . . . . . . . 14

2 CellTracks TDI - an Image Cytometer for Cell Characterization 19

2.1 Introduction . . . . . . . . . . . . . . . . . . 20

2.2 Materials and Methods . . . . . . . . . . . . . . . 20

2.2 .1 Beads . . . . . . . . . . . . . . . 20

2.2.2 CTC positive samples . . . . . . . . . . . . 21

2.3 Instrumentation . . . . . . . . . . . . . . . . . . . . . . . . . . . . . . . . . . . .

2.3.1 Optical System . . . . . . . . . . . . . . 21

2.3.2 Beam homogenizer .............. 24

2.3.3 Feed Forward Focusing . . . . . . . . . . . . . . . 24

2.3.4 Sample scanning ............... 26

2.3 .5 TDI camera . . . . . . . . . . . . . . . . 26

2.3.6 Discrete Final Magnification . . . . . . . . . . 27

2.3.7 Instrument control . . . . . . . . . . . . . . . . . 28

2.3 .8 Image Analysis . . . . . . . . . . . . . . . . . . 28

2.4 Results . . . . . . . . . . . . . . . . . . . . 30

2.4 Illumination . . . . . . . . . . . . . . . . 30

2.4 .2 Resolution ................. . . . . 30

2.4.3 Acquisition and analysis time . . . . . . . . . 33

2.4.4 Performance ............... . . 34 
2.4 .5 CTC Imaging . . . . . . . . . . . . . . . . . . . . . . 35

2.5 Discussion . . . . . . . . . . . . . . . . . . . . 38

2.6 References ..................... 40

3 Automated Identification of Circulating Tumor Cells by Image Cytometry $\quad 43$

3.1 Introduction . . . . . . . . . . . . . . . . . . . . . 44

3.2 Materials and Methods . . . . . . . . . . . . . . . . . . . . . . 44

3.2.1 Patients and controls . . . . . . . . . . . . . . . . 44

3.2.2 Sample preparation . . . . . . . . . . . . . 44

3.2.3 CellTracks Analyzer II . . . . . . . . . . . . . . . . . . . . . . . . 45

3.2.4 CellTracks TDI analyzer . . . . . . . . . . . . . . 45

3.2.5 Automated Event Classification . . . . . . . . . . . 46

3.3 Results . . . . . . . . . . . . . . . . . . 47

3.3.1 CellTracks TDI data analysis . . . . . . . . . . . 47

3.3.2 Inter and intra operator variability . . . . . . . . . 53

3.3.3 Comparison of CTC analysis by CellTracks Analyzer II and CellTracks TDI . . . . . . . . . . . . . . . 55

3.4 Discussion . . . . . . . . . . . . . . . . . . . . . 57

3.5 Acknowledgements . . . . . . . . . . . . . . . . 60

3.6 References ...................... 61

4 Development and characterization of cell alignment struc$\begin{array}{ll}\text { tures } & 65\end{array}$

4.1 Introduction . . . . . . . . . . . . . . . . 66 66

4.2 Materials and Methods . . . . . . . . . . . . . . 67

4.2.1 Design of cell alignment microstructures . . . . . . . 67

4.2.2 Cleanroom technologies for wafer fabrication . . . . 70

4.2.3 Production of microstructures on silicon wafers . . . 73

4.2.4 PDMS imprinting . . . . . . . . . . . 74

4.2.5 Preparation of cell analysis cartridges using PDMS chips . . . . . . . . . . . . . . 77

4.2.6 Cancer cell line samples . . . . . . . . . . . . . . . 79

4.3 Results . . . . . . . . . . . . . . . . . . 79

4.3.1 PDMS imprinting of microstructures . . . . . . . 79

4.3.2 Image quality of cells imaged in the microstructures $\quad 80$

4.3.3 Capture efficiency of cells on the viewing surface of the microstructures . . . . . . . . . . . 82

4.3.4 Alignment efficiency of the microstructures . . . . 85

4.3.5 Fluorescence detection efficiency of beads in the mi-

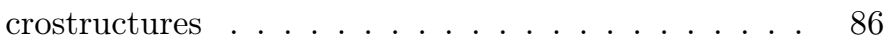

4.4 Discussion . . . . . . . . . . . . . . . . . . . . . . . . . . . . . . . . . . . 87

4.5 References ..................... 89 
5 Implementation of cell alignment structures in CellTracks TDI 91

5.1 Introduction . . . . . . . . . . . . . . . . . . . 92

5.2 Materials and Methods . . . . . . . . . . . . . . . 92

5.2.1 Sample preparation . . . . . . . . . . . . . . 92

5.2.2 Cell presentation cartridges . . . . . . . . . . . 93

5.2 .3 CellTracks TDI system . . . . . . . . . . 93

5.3 Results . . . . . . . . . . . . . . . . . . . . . . . 95

5.3.1 Scanning of microstructured cartridges . . . . . . . 95

5.3.2 Autofocus on different positions . . . . . . . . . . . . . 95

5.3.3 Interpolation of focus positions . . . . . . . . . . . 97

5.3.4 Control software . . . . . . . . . . . . . . 99

5.3.5 Improvements in scan and analysis times . . . . . 101

5.3.6 Image quality comparison with and without unbound ferrofluid . . . . . . . . . . . . . 103

5.3.7 Linearity of recovery using SKBR-3 cells . . . . . . . 104

5.4 Discussion . . . . . . . . . . . . . . . . . . . . 104

5.5 References ..................... 107

6 Quantitative and qualitative effect of free ferrofluid on cell analysis

6.1 Introduction . . . . . . . . . . . . . . . . . . 110

6.2 Materials and Methods . . . . . . . . . . . . . . . . . 110

6.2.1 Fluorescent magnetic beads . . . . . . . . . . . . 110

6.2 .2 Leukocytes . . . . . . . . . . . . . . . 111

6.2.3 Cancer cells . . . . . . . . . . . . . . 112

6.2.4 CellTracks TDI . . . . . . . . . . . . . . 113

6.2.5 Event detection in CellTracks TDI images . . . . . . 113

6.3 Results . . . . . . . . . . . . . . . . . . . . . 114

6.3.1 Effects of free ferrofluid on fluorescence measurements 114

6.3.2 Effects of free ferrofluid on imaging of leukocytes . . 115

6.3.3 Effects of free ferrofluid on imaging of cells from the breast cancer cell line SKBR-3 . . . . . . . . . . 117

6.3.4 Comparison of effects across all object types . . . . . 119

6.3.5 Determination of the cell surface membrane with a bright-field image . . . . . . . . . . . . . . 121

6.4 Discussion . . . . . . . . . . . . . . . . . . . 123

6.5 References .................... 126

7 Removal of free ferrofluid after immunomagnetic enrichment of CTC 129

7.1 Introduction . . . . . . . . . . . . . . . . . 130

7.2 Materials and Methods . . . . . . . . . . . . . . . 130

7.2.1 SKBR-3 cells in buffer . . . . . . . . . . . . 130 
7.2.2 SKBR-3 and PC3-9 cells spiked in whole blood samples from healthy donors . . . . . . . . . . . . . 131

7.2.3 Blood samples from carcinoma patients . . . . . . . 131

7.2.4 Measurement of free ferrofluid concentration . . . . 131

7.2.5 CellTracks AutoPrep . . . . . . . . . . . . . . 132

7.2.6 Automated ferrofluid removal setup (AFRS) . . . . . 133

7.2.7 CellTracks Analyzer II and CellSpotter . . . . . . . 137

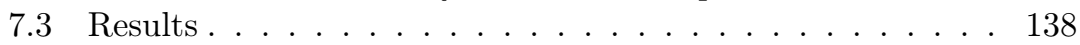

7.3.1 Simulation of particle movement in conical tube . . 138

7.3.2 Determination of free ferrofluid removal efficiency . . 145

7.3.3 SKBR-3 cells in buffer . . . . . . . . . . . . . . 145

7.3.4 SKBR-3 and PC3-9 cells spiked in whole blood . . . 147

7.3.5 Samples from carcinoma patients . . . . . . . . . . 148

7.4 Discussion . . . . . . . . . . . . . . . . . . . . 150

7.5 References ..................... 154

A Overview of clean-room processing steps 155

Summary $\quad 159$

Conclusions . . . . . . . . . . . . . . . . . . . . . 159

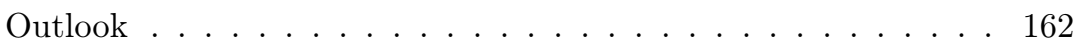

Samenvatting $\quad 165$

Conclusies . . . . . . . . . . . . . . . . . . . 165

Vooruitzichten . . . . . . . . . . . . . . . 168

$\begin{array}{ll}\text { List of publications } & 171\end{array}$

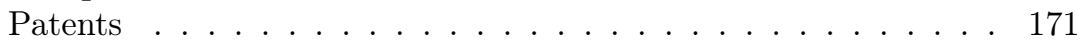

Journal articles . . . . . . . . . . . . . . . . . . . . 171

Conference contributions (oral) . . . . . . . . . . . . 171

Conference contributions (poster) . . . . . . . . . . . . 171

Article in proceedings . . . . . . . . . . . . . . 172

$\begin{array}{ll}\text { Dankwoord } & 175\end{array}$

$\begin{array}{ll}\text { About the author } & 177\end{array}$ 


\section{CHAPTER}

\section{INTRODUCTION}

\subsection{INTRODUCTION TO CANCER}

Since its first known documented description, around 1500 B.C., a lot has changed about the knowledge we have of cancer. The first documented case describes 8 patients with breast cancer that were treated by cauterization, not the most patient friendly method. There was assumed to be no treatment that could cure the patient at that time.

A lot has changed since then. More delicate procedures have replaced the early primitive methods of removing the cancer. Next to surgery, current options include chemotherapy, radio therapy, bone marrow transplant and targeted therapies that use specific treatment targets to attack cancer cells while minimizing their destructive effect on normal cells.

More knowledge has also been gained about the process of the disease and the best ways to prevent recurrence in patients that have had surgery to remove the primary tumor. A major current theory suggests that normal cells have to acquire six main capabilities in order to become cancerous cells [1]. One of these is the capability to invade tissue and form metastases. This capability allows the cancerous cells to leave the primary tumor and enter the blood stream to proliferate at a distant location where nutrients and space are more abundant. Growth of new tumors at distant locations, termed metastasis, is the cause of death for $\sim 90 \%$ of cancers [2]. The metastatic process was first demonstrated in 1869 [3]. In 1889, the "seed and soil" theory was proposed which states that certain tumor types tend to metastasize to specific organs [4]. Most of the cells that are shed into the bloodstream by the primary tumor are removed by the body's defense mechanisms. Remaining cells can either form distant metastases or remain dormant, sometimes for years, to form secondary tumors, up to decades, later. See Figure 1.1. 


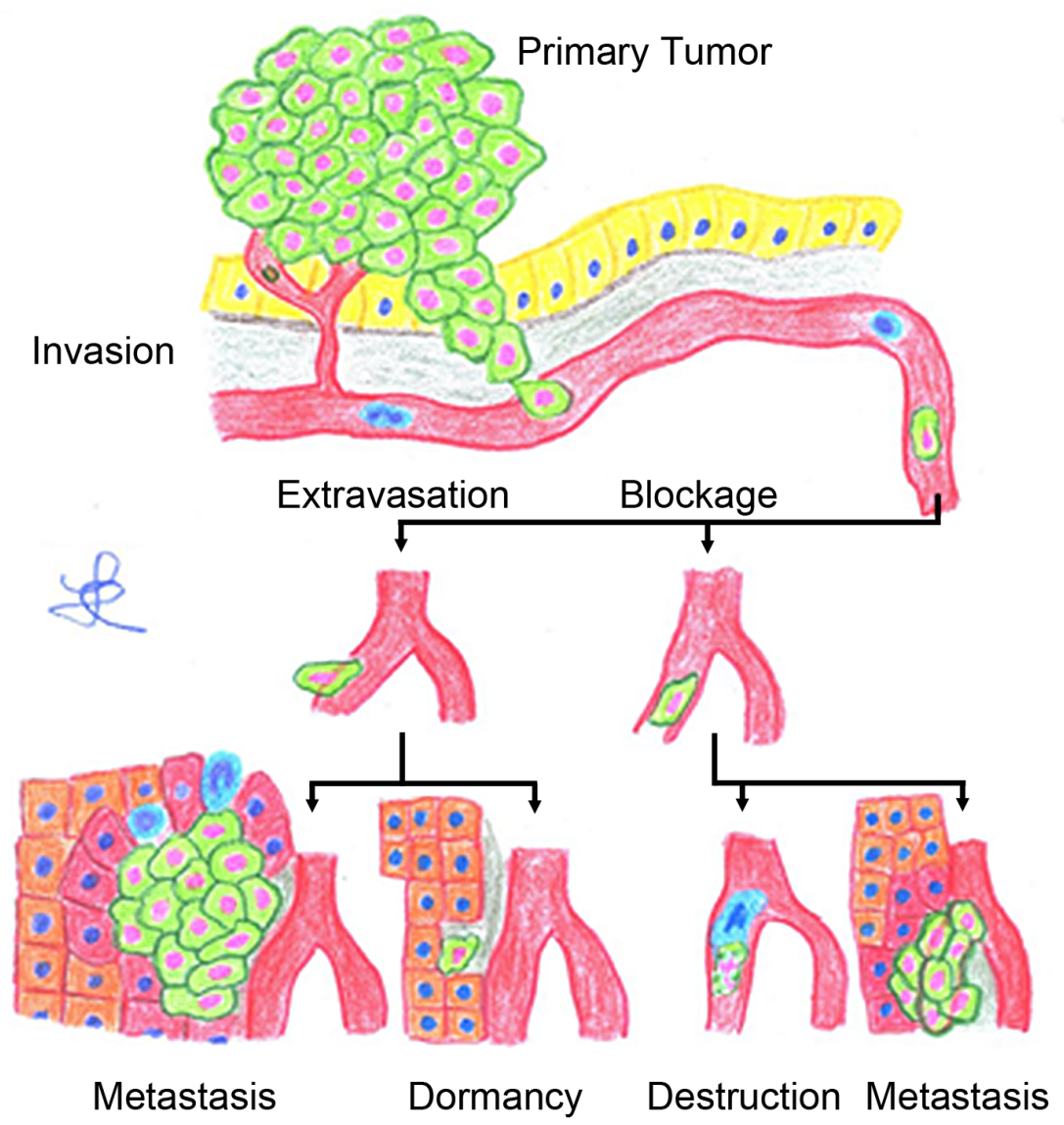

FIgURE 1.1 : Artistic impression of the metastatic pathway. Neovascularization causes local blood vessels to grow in the tumor, providing vital nutrients for the tumor to expand. Once cells in the tumor have acquired the capability to invade tissue, they can invade the blood stream. Cells that have entered the blood stream can either undergo extravasation, resulting in metastasis or dormancy, or be blocked by a blood vessel with a small diameter. Blocked cells can then also form metastases or they can be destroyed by the body's immune system. Illustration by Prof. Dr. Leon Terstappen. 
Although a lot is known about cancer and the processes involved in metastasis, the number of people that die from cancer is still very high. In 2012 alone, a total number of 1.64 million new cases of cancer are expected to occur in the United States alone [5]. The expected number of deaths in the USA in 2012 due to cancer is 577000 . The most frequent types of new cancers are prostate cancer in men $(29 \%)$ and breast cancer in women (29\%). The second and third most likely types of newly developed cancers are lung cancer (14\%) and colon cancer (9\%) in both sexes. While the greatest number of people are expected to die from lung cancer $(29 \%$ in men and $26 \%$ in women).

Averaged over the years 2006 - 2008, the total probability of developing any type of cancer in a lifetime is $45 \%$ in men and $38 \%$ in women. On a positive note, the combined male and female mortality rate of cancer has slowly but steadily decreased during the past 20 years. This is most likely due to better surgical procedures that are applied to remove the primary tumor and better hormone- and chemotherapy to minimize the effect of minimal residual disease (MRD). However, the mortality rate for heart disease in the USA has decreased much more rapidly. So much so that since 1999, the death rates for heart disease have been lower than those for cancer, in people younger than 85 years. In people that are older than 85 , heart disease is still approximately 3 times more deathly than cancer. The combination of cancer being the number 1 cause of death and the fact that most people die from metastases makes it an area of large and continued interest for researchers.

\subsection{The use of Circulating tumor Cells to improve TREATMENT DECISIONS IN THE CLINIC}

In all but one pathway of tumorigenesis that has been proposed [1] the final acquired capability is tissue invasion and metastasis. For this capability to occur, several classes of proteins have to be altered, a major class being cell-cell adhesion molecules. One of these is the Epithelial Cell Adhesion Molecule (EpCAM). This epithelial transmembrane glycoprotein is present on normal epithelial tissue and a large fraction of human tumor types [6]. It connects epithelial cells in organs and tissues and is normally not found on cells which circulate in the human bloodstream. When a cell is found in the bloodstream that exhibits this molecule, it is said to be a circulating epithelial cell, or circulating tumor cell (CTC).

Since CTC are the main cause of metastasis and metastases are the main cause of death in cancer patients, it is of clinical importance to be able to detect CTC with high specificity and selectivity. Not only due to the fact that CTC are in most cases extremely rare; just a few CTC are typically found in $1 \mathrm{ml}$ of blood of a cancer patient among $\sim 7$ million leukocytes and $\sim 5$ billion erythrocytes. But also due to the fact that even a small 
number of CTC present in the bloodstream significantly affects survivability. Several clinical studies have shown the relationship between the presence of CTC and progression-free and overall survival in different types of cancers: In metastatic colorectal cancer (MCRC, [7]), in metastatic breast cancer (MBC, $[8,9])$, in castration-resistant prostate cancer (CRPC, [10]) and in non-small-cell lung cancer (NSCLC, [11]). In all studies, the detection of CTC provides a powerful prognostic value and the detected concentration of CTC can be used as a surrogate marker to stratify patients in low and high risk categories.

In the CellSearch system (Veridex, Raritan, NJ, USA), currently the only FDA approved system for clinical CTC detection, patients with MBC and $\mathrm{CRPC}$ are stratified into favorable and unfavorable risk groups based on a cut-off concentration of 5 CTC per $7.5 \mathrm{ml}$ of blood [8, 12]. For patients with colorectal cancer the cut-off has been set at $3 \mathrm{CTC}$ per $7.5 \mathrm{ml}$ of blood [7]. Patients in the unfavorable group have significantly lower median overall- and progression-free survival. The CellSearch system was validated for routine assessment of blood samples from $\mathrm{MBC}$ patients in the clinical laboratory in a multicenter trial [13].

In a recent study [14], the use of CTC in determining overall survival in $\mathrm{MBC}$ patients was compared to radiology, which is currently the gold standard. The presence of CTC correlated better with overall survival when compared to current radiological imaging methods in patients with MBC. CTC enumeration proved to be a more reproducible method and a more robust predictor of survival that can be used at an earlier time point as compared to radiographic response. Reproducibility was determined by way of inter-reader variability, which was $15 \%$ in radiological imaging and $1 \%$ in CTC enumeration. This indicates that incorrect disease status determinations with the use of CTC as a measure of treatment efficacy would be less likely, thereby reducing the frequency of incorrect treatment decisions. Furthermore, changes in CTC concentrations in MBC patients during chemotherapy have been shown to correlate with overall survival [15]. Patients that had decreasing levels of CTC survived significantly longer than patients that had increasing levels of CTC $(17.7 \pm 5.9$ against $4.5 \pm$ 0.5 months). The use of CTC as a determination of treatment efficacy is currently exploited in several clinical studies. One example is the SWOG S0500 phase III trial [16], in which an elevated level of CTC in the blood of MBC patients at first follow-up is used to randomly maintain or change the therapy that is given. This interesting study therefore directly investigates the ability of CTC enumeration to successfully guide therapy in cancer patients. A significant correlation between a change in therapy, when CTC levels do not decrease using the first therapy, and an increase in progression free- or overall survival will most likely accelerate the further implementation of CTC in the clinic as the new gold standard. 


\subsection{Molecular therapy targets Related to CTC}

Specific treatment targets exist on various types of cancers, and CTC from those cancers, which are of interest in the clinic. They provide a possibility to tailor treatment of a patient to the specific properties of the cancer that a patient has. Personalized treatment has already improved overall survival (OS) and progression-free survival (PFS) and will reduce the occurrence of patients receiving generic treatment.

One well documented and researched treatment target is the Human Epidermal growth factor Receptor 2: HER2/neu. Which is a proto-oncogene and is one of the most frequently altered in human cancers [17]. The HER2 gene, which encodes the growth factor receptor HER2, is amplified and HER2 is thereby over-expressed in 25 to 30 percent of breast cancers, increasing the aggressiveness of the tumor [18]. HER2 over-expression can make cancer cells resistant to apoptosis and thereby increase the propensity of the tumor to grow. Also, amplification of the HER2 gene has a significant prognostic value for OS and PFS in breast cancer. Its prognostic value is on par with the number of positive lymph nodes detected [19]. Moreover, HER2 gene amplification in localized (lymphnode-negative) cancers proved to be an independent predictor of poor clinical outcome and was an even stronger discriminant than tumor size [20].

After HER2 seemed to be involved in pathogenesis of cancer, monoclonal antibodies have been developed directed against its extracellular domain. One prime example is trastuzumab (Herceptin ${ }^{\circledR}$ ), which was humanized from a murine antibody [21]. Trastuzumab has been the subject of many clinical studies and was shown to have a significant positive impact on PFS and OS in patients with MBC by inhibiting tumor growth. It also had synergistic effects when combined with chemo-therapy [18, 22], further improving PFS and OS. In 1998, Herceptin was approved by the FDA for use in MBC patients, and in 2006 also for the use in patients with early stage breast cancer and has since saved or improved the lives of many women with breast cancer.

Another promising treatment target, for CRPC patients, is cytochrome$\mathrm{P}$ (CYP) 17, which is a key enzyme in androgen synthesis. Clinical trials of abiraterone acetate, which inhibits CYP 17, have shown that it is safe and has significant anti-tumor activity in CRPC [23]. Also, in a phase II clinical trial, significant decreases in CTC count in CRPC patients were observed [24]. Further characterization of genes, by means of FISH (Fluorescence in situ hybridization) of ERG, AR and PTEN, found in CTC from CRPC [25], resulted in improved understanding of CRPC.

Since cancers in patients change, not only in size, but also in the expression of treatment targets even during treatment, an accurate and real-time method is needed. Taking a real biopsy of the tumor is not feasible on a repeated basis. The ease of use of CTC and all the information about the tumor they contain can solve this issue. The ability to enumerate CTC 
and accurately characterize specific treatment targets on CTC is therefore very useful in the clinic as it gives a real-time biopsy.

Presence of specific treatment targets on CTC, as described above, can be visualized by various fluorescence technologies. A CTC detection technology with the possibility of combined detection of multiple treatment targets in a single CTC or the specific detection of treatment targets on single CTC will greatly improve the value of that technology in the clinic.

\subsection{CTC Detection TeChNologies}

CTC present an interesting new target for the staging of various types of cancers. The accurate detection of CTC will most likely assist treatment decisions in the clinic in coming years. However, the accurate detection of CTC in human peripheral blood is not easy. CTC are extremely rare and most detection technologies use a combination of enrichment and identification steps to enumerate and characterize them.

Enrichment of CTC is most often based on antibody binding and size. Antibodies against the epithelial cell adhesion molecule (EpCAM) are used to positively enrich CTC from other blood cells. Detection of CTC is often based on antibodies against cytokeratins (CKs), which are proteins that make up the intermediate filaments in the cytoskeleton of epithelial tissue. There are a dozen known and well documented CK types [26]. CK's 8, 18 and 19 are often used in CTC research to distinguish epithelial cells from hematopoietic cells. Depletion of white blood cells, with the use of antibodies against CD45, is also used to achieve enrichment of CTC. An advantage of this method is that CTC that have no, or no detectable, EpCAM antigen expression are also detected by this method, as opposed to a method that uses EpCAM antibodies to enrich CTC. This is especially useful in the detection of cells that have undergone an epithelial to mesenchymal transition (EMT). Cells that have undergone such a transition have significantly altered expression of antigens, causing a possible reduction in the expression of EpCAM on their cell surface. CTC detection technologies that use EpCAM for enrichment of detection of CTC might therefore underestimate the real number of CTC in a patient. EMT is the subject of current investigation and it appears that cells that have undergone this transition have properties similar to stem cells [27].

A second property of CTC that is used during enrichment is their size. On average, CTC are larger than red- and white blood cells. Although one has to note that cultured cells, which are often used during initial testing of a new method, are often significantly larger than CTC found in the human bloodstream. This might therefore produce skewed results for methods which only use cultured cells for testing and device optimization. One method that uses size to enrich CTC is the ISET method (isolation by size of epithelial tumor cells) [28, 29]. This method uses polycarbonate Track- 
etch membrane filters with $8 \mu \mathrm{m}$ diameter cylindrical pores. Peripheral human blood is diluted 10 times and run through the filter using vacuum aspiration. Cells that remain on the filter are stained with a pan-CK antibody (KL1), which specifically binds to epithelial CKs. In addition, certain cells of interest can be extracted by laser microdissection and analyzed further for specific DNA abnormalities. This method has been used to show presence of CTC in 12 of $44(27 \%)$ of MBC patients. Another method that uses size to enrich CTC from whole blood is developed in our group. Track-etched polycarbonate filters with diameters ranging from 5 to $10 \mu \mathrm{m}$, and microfabricated silicon nitride filters with the same diameter range were tested using several cultured cell types [30]. Ideal filter properties for CTC enrichment were determined to be, among others, hole size of $5 \mu \mathrm{m}$, thickness of at least $10 \mu \mathrm{m}$ and a stiff and flat material that does not interact with cells. In the near future, this method will be tested on blood samples from cancer patients to validate the performance of the ideal filter. An advantage of these filtration methods is that they can also detect EpCAM negative cells, while a disadvantage is that CTC smaller than a certain size will pass through the filter, lowering the sensitivity.

Several technologies have been developed and tested which use a combination of antigens to enrich and detect CTC. One of these is the "CTCchip" [31]. This technology uses anti-EpCAM coated microposts to capture CTC flowing through a microfluidic chip. Anti-CK is used to identify CTC and anti-CD45 is used to identify white blood cells. This technology showed rather high presence of CTC in a particular study with 115 of 116 (99\%) patient samples containing at least $5 \mathrm{CTC} / \mathrm{ml}$. The median concentration of $\mathrm{CTC} / \mathrm{ml}$ detected in this study is significantly higher than reported in other methods, although no CTC were reported in 20 samples from healthy controls. A recent improvement to this system [32] was introduced which increases the interaction between cells and anti-EpCAM labeled microposts. However, results indicate that up to $10 \mathrm{CTC} / \mathrm{ml}$ were detected in samples from healthy controls. This might warrant further investigation as to whether the detected cells are in fact CTC. A second technology that uses antibody binding to detect CTC is the "FAST" system [33, 34]. Here, a fiber-optic scanning method with a wide field of view is used to determine the fluorescence signal intensity of a large sample area containing mononuclear cells from lysed whole blood. Cells of interest are re-analyzed, using a $40 \times$ microscope objective, for pan-CK staining and DAPI. In a study of MBC patients, CTC were found in 12/14 (86\%) of patients. A similar technology to the FAST system is the MAINTRAC system. In this technology, whole blood is lysed and stained using anti-EpCAM coupled to fluorescein isothiocyanate (FITC) and CD45 coupled to phycoerythrin (PE) [35-37]. The sample is then applied to an adhesion slide and scanned using a laser scanning cytometer. Events of interest can be relocated for further examination. This technology has been used to detect CTC in patients with varying stages of breast and lung cancer. In total, 92 of 100 
patients $(92 \%)$ had CTC.

A variety of new $\mathrm{CTC}$ detection technologies have been introduced recently, each using different definitions for what constitutes a CTC. This has lead to a large range of reported CTC concentrations in patients. Detection technologies that use less strict definitions usually detect higher CTC concentrations in patients. However, care has to be taken to also carefully determine the CTC concentration that is detected by such a technology in healthy controls and patients with benign disease to assure a high specificity of CTC detection. Ideally, a technology is required that standardizes the process of CTC enrichment, detection and enumeration and which is also extensively tested in healthy controls and patients with benign disease. The CellSearch system (Veridex, Raritan, NJ, USA) is such a CTC detection technology and it is strongly related to this thesis. Its specifics and associated systems will be described in the next section.

\subsection{The CellSearch System}

The CellSearch system uses a combination of antibodies to enrich and detect CTC and is FDA approved for the detection of CTC in metastatic breast-, metastatic prostate- and metastatic colorectal cancer patients. It consists of 3 main components: the CellSave preservative tube, CellTracks AutoPrep and CellTracks Analyzer II. The CellSave preservative tube is used to collect whole blood samples from patients by venipuncture. A preservative in the tube stabilizes the blood up to 96 hours and allows for storage or shipment of samples to remote locations. Blood samples are subsequently processed by the CellTracks AutoPrep system for the immunomagnetic enrichment and immunofluorescence labeling of CTC. Processed samples are imaged by the CellTracks Analyzer II and selected images of possible CTC are scored by a trained reviewer.

\subsubsection{CELLTRACKS AUTOPREP}

The CellTracks AutoPrep is an automated system consisting of nine (pipetting) stations that each perform specific tasks to enrich and label CTC for a maximum of 8 samples. First, $7.5 \mathrm{ml}$ of blood from the CellSave preservative tube is transferred to a $15 \mathrm{ml}$ conical tube for each sample and $6.5 \mathrm{ml}$ dilution buffer is added. The conical tubes are then centrifuged at $800 \mathrm{~g}$ for 10 minutes and placed inside the AutoPrep system. Each sample is then processed in series by the 9 stations. At the first station, the plasma is aspirated and EpCAM-Ferrofluid and buffer are added and the sample is mixed. In the next 2 stations the sample is incubated with the ferrofluids. Moving external magnets are used to force the ferrofluid through the sample to improve cell loading. Then, the sample is washed twice while all magnetically labeled objects are retained with the use of a high gradient magnetic field. After these wash steps, 4',6-diamidino-2-phenylindole (DAPI, to stain 
the DNA), Cytokeratin-PE (against cytokeratins 8, 18 and 19, to stain the cytoskeleton in epithelial cells (CK-PE)), CD45-APC (to counterstain white blood cells) and a permeabilization reagent are added and the sample is incubated in the next station. The sample is then washed again with buffer while the magnetically labeled objects are retained. The next station allows the cells in the sample to settle and the last station then removes the top part of the sample which only contains free ferrofluid. The sample is then washed for the last time and the cells that remain in the sample are fixed and transferred to a CellSearch cartridge, which is already inside a CellTracks Magnest. A schematic overview of the AutoPrep stations is shown in Figure 1.2.

\subsubsection{CELLTRACKS MAGNEST}

The CellTracks Magnest is an assembly of an iron yoke containing two wedge-shaped magnets. It is designed to present all magnetically labeled objects from a sample at an analysis surface in a homogeneous layer. [38]. A cartridge containing the sample in a rectangular cuvet is inserted in the assembly, under the magnets, and held in place by a support structure. See Figure 1.3.

The wedged shape of the magnets generates a large magnetic gradient in the vertical direction. This forces magnetically labeled objects in the cuvet to the top, where they accumulate at the upper surface after an incubation time of 20 minutes. The horizontal component of the magnetic gradient in the space occupied by the cuvet is minimized to prevent horizontal movement of magnetically labeled objects. This results in a homogeneous distribution of cells at the upper surface of the cartridge which is now imaged by the CellTracks Analyzer II.

\subsubsection{CELLTRACKS ANALYZER II}

The CellTracks Analyzer II is a computer controlled epi-fluorescence microscope that is specifically build to image the upper surface of an analysis cartridge in a CellSearch Magnest. It uses a $100 \mathrm{~W}$ mercury arc lamp, 4 filter cubes, a $10 \times / 0.45 \mathrm{NA}$ microscope objective and a 12 -bit CCD camera to image the cells. Fluorescence channels are optimized for imaging of DAPI, PE, FITC and APC. Automated imaging of the cartridge starts with detection of the edges of the cartridge and automatic focusing on objects in the DAPI channel. The entire upper surface of the cartridge is then imaged for all 4 channels using a pixel size in the object space of $0.65 \mu \mathrm{m}^{2}$, resulting in typically 175 images for each fluorescence channel. Next, an automated image analysis algorithm then selects objects which are positive for both DAPI and CK-PE. An expert reviewer then scores a gallery of images of the selected objects using the CellSearch CTC definition to identify CTC. The CellSearch CTC definition states that an object is to be counted as 

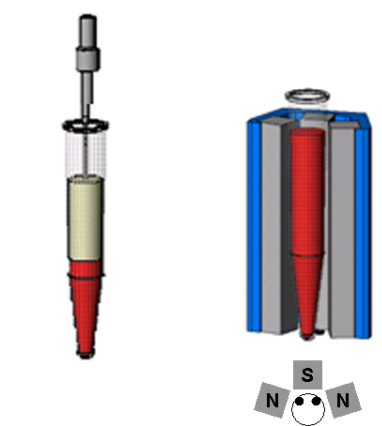

Station 1 Station 2
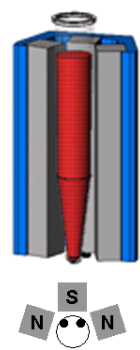

Station 3

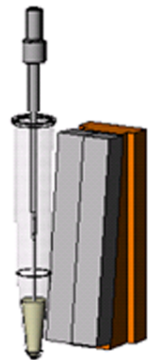

S N

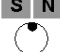

\section{Station 4}

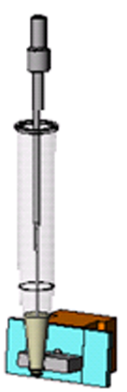

S N

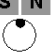

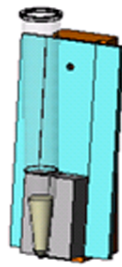

S $\mathbf{N}$

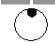

Station 5
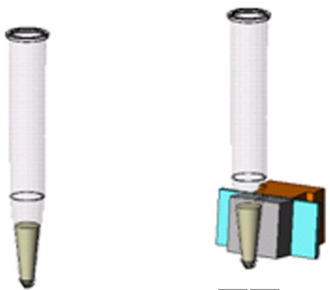

S $\mathbf{N}$

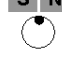

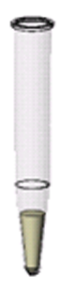

Station 8

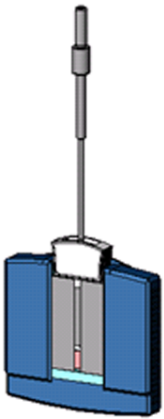

Station 9

FIGURE 1.2 : Schematic overview of the 9 stations in the CellTracks AutoPrep system. The position of the external magnets used in stations 2, 3, 4, 5, 7 and 9 is indicated by the small top-down view drawings. $\mathrm{N}$ and $\mathrm{S}$ indicate magnetic poles and the small black circles indicate the position of the magnetically labeled objects in the tube. Station 1 aspirates the plasma and adds EpCAM-Ferrofluid. Stations 2 and 3 both perform magnetic incubation and both use moving external magnets to improve cell loading. Station 4 performs magnetic separation and re-suspension. Station 5 adds the fluorescent dyes DAPI, CK-PE, CD45-APC and optional markers coupled to FITC. Stations 6-8 perform incubation, magnetic wash and ferrofluid reduction. Station 9 performs re-suspension and transfers the samples to a cartridge that is inside a Magnest. 

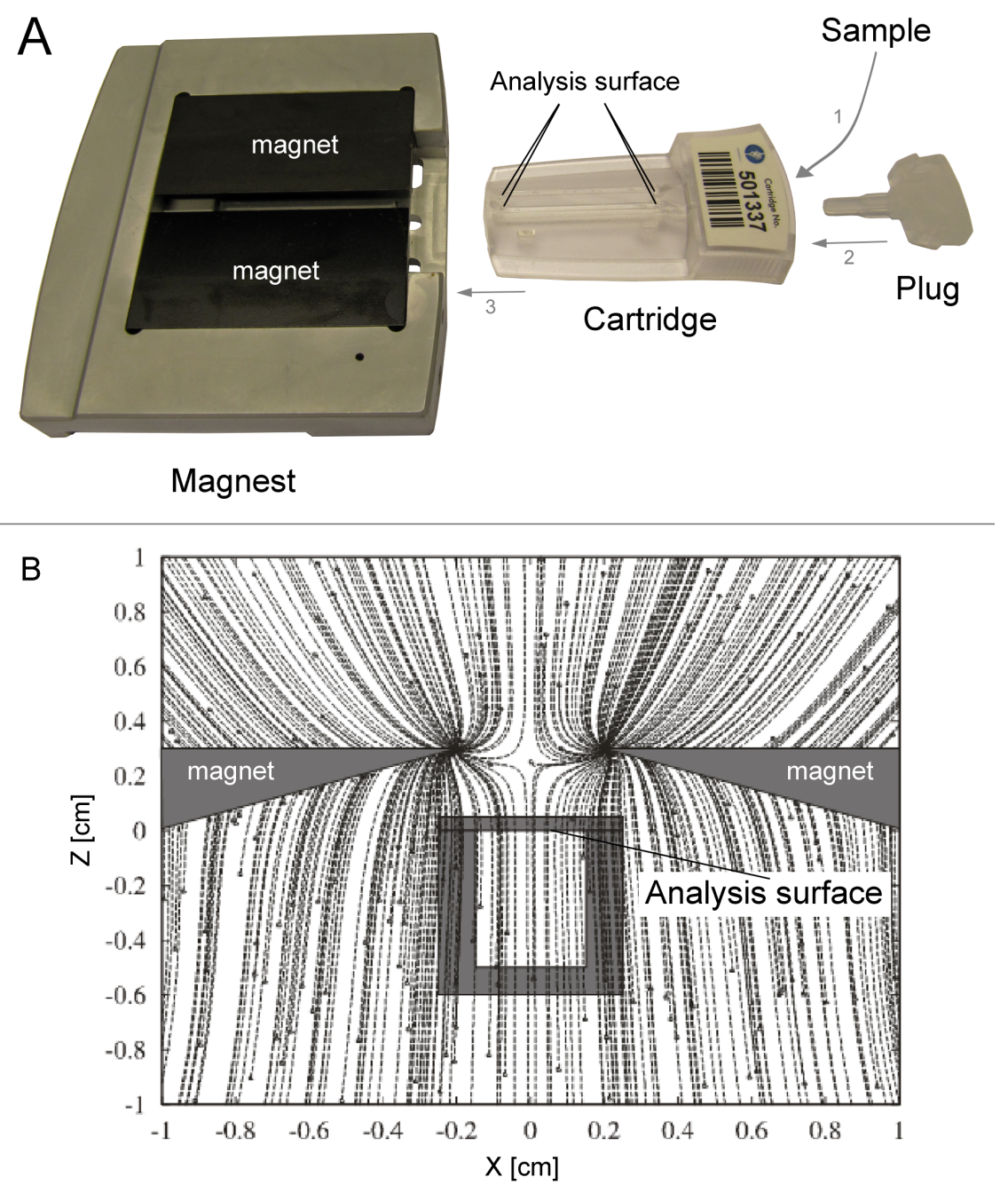

Figure 1.3 : Overview of CellSearch Magnest system that is used to collect magnetically labeled cells at an analysis surface. (A) The sample $(360 \mu \mathrm{l})$ is introduced into the cartridge (arrow 1) generally with the use of a pipette. A plug is used to seal the sample inside the cartridge (arrow 2) and the cartridge is inserted into the Magnest (arrow 3). (B) Dotted lines show simulated movement of cells under the influence of the magnetic gradient caused by the 2 magnets. Cells in the cartridge are homogeneously distributed at the analysis surface after an incubation time of 20 minutes. 
a CTC when it has the morphology of a cell, is both DAPI and CK-PE positive, is negative for CD45-APC, is larger than $4 \times 4 \mu^{2}$ and for which the nucleus is inside the cytoplasm for more than $50 \%$. A gallery containing various cell types found in samples from cancer patients and imaged by the CellTracks Analyzer II is shown in Figure 1.4.

The objects in Figure 1.4 illustrate that there is a large heterogeneity in CTC morphology and objects resembling CTC. Because these images are scored by a human reviewer and due to the elaborate CellSearch definition, there is significant intra- and inter user variability. In a recent multilaboratory study of the CellSearch system [39], objects that were detected by the CellTracks Analyzer II in 6 samples were scored by 14 independent reviewers. Discordant classification of objects occurred in $4-31 \%$ (median $14 \%$ ) of cases. Image interpretation contributed significantly to the interlaboratory variation, especially in samples with high numbers of apoptotic cells. Furthermore, simulations have shown that if the classification error can be reduced to zero, the threshold of 5 CTC / $7.5 \mathrm{ml}$ blood will go to 1 CTC / $7.5 \mathrm{ml}$ of blood [40].

\subsection{Challenges AdDressed in this Thesis}

- The sensitivity of detection and ability to quantify antigen expression of the CellTracks Analyzer II is limited due to trade-offs between spatial resolution, speed of data collection, storage requirements, image analysis, and the wish to keep the total analysis time acceptable for routine applications. Also, the emission spectrum of the mercury arc lamp used in the CellTracks Analyzer II is not ideal for the excitation of APC. Granulocytes, which are also found in samples that are processed by the CellTracks AutoPrep, have lower CD45 expression than lymphocytes. Inefficient excitation of CD45-APC therefore results in these cells appearing to be CD45 negative, resulting in errors in CTC enumeration.

- Manual review of cells, for presence of CTC, using the CellSearch definition is the main cause of inter-laboratory variation. Also, studies have shown that all $\mathrm{EpCAM}^{+} \mathrm{CK}^{+} \mathrm{CD} 45^{-}$objects, not just the CTC from the CellSearch definition, predict overall survival and have clinical importance [41]. An automated method that quantifies not only CTC, but also apoptotic CTC and CTC fragments will remove reviewer error and expand the scope of detected objects.

- Alignment and concentration of cells at the analysis surface reduces imaging times. In the early CellTracks system this was accomplished by the use of thin ferromagnetic nickel lines at the analysis surface [42]. However, this system was not specifically designed for the detection of rare cells, such as CTC. Parts of the aligned cells can be blocked by the nickel lines and this is not acceptable for detection of CTC. A different strategy is required to align and concentrate cells in samples that contain CTC enabling 


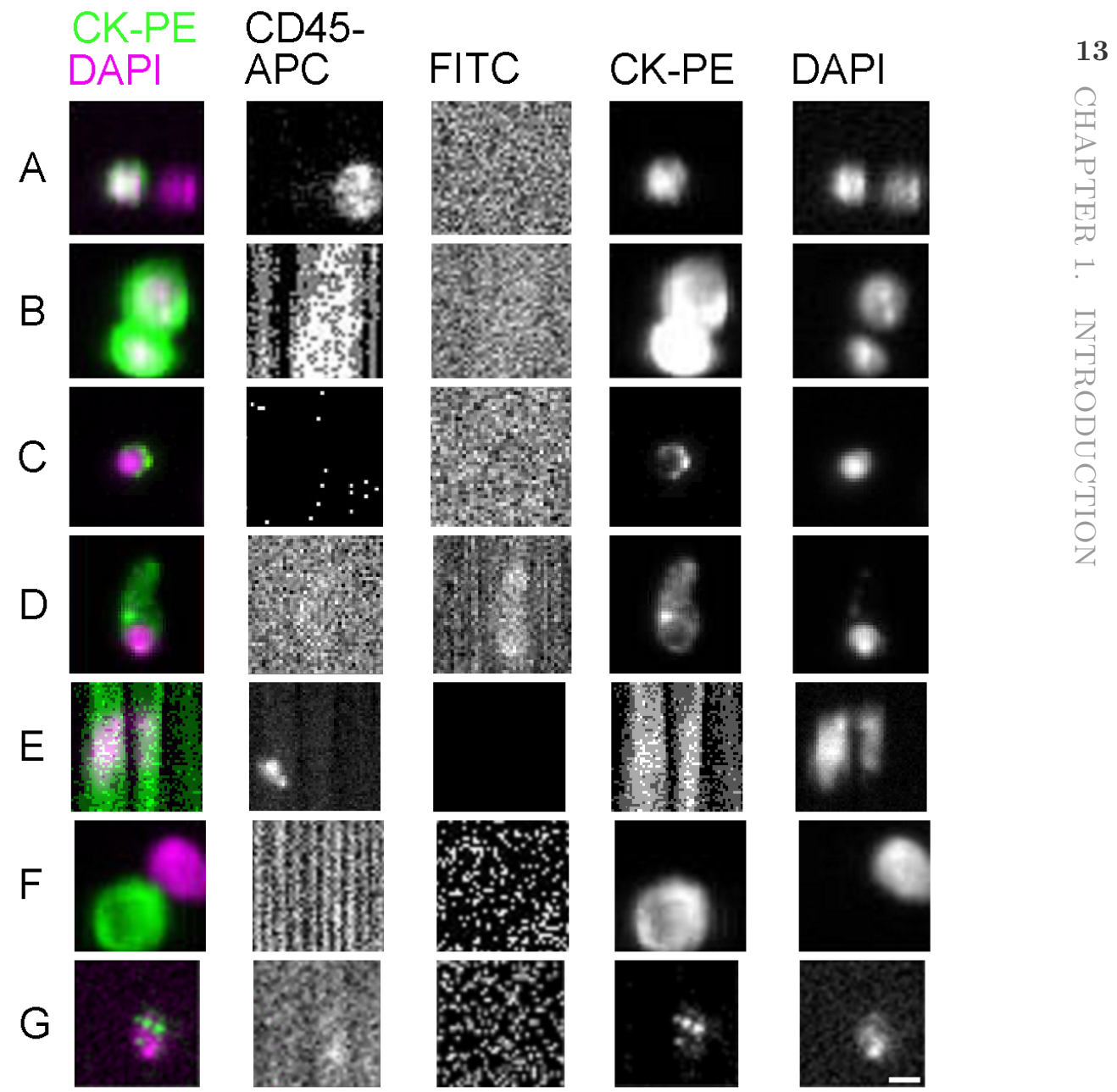

FigURE 1.4 : Gallery of various cell types found in samples from cancer patients imaged by CellTracks Analyzer II. First column shows overlay of DAPI (Nucleus, purple) and CK-PE (Cytoplasm, green) and remaining columns show images of separate fluorescence channels. (A) CTC neighbored by a WBC, (B) two CTC side-by-side, (C) small CTC with barely enough cytoplasm to make the CellSearch CTC definition, (D) CTC with elongated cytoplasm, (E) Aggregates of ferrofluid obstruct DAPI and PE fluorescence images, (F) cytoplasm without or with displaced nucleus and (G) apoptotic CTC. Only the cells in panels A-D were classified as being CTC by an expert reviewer. Scale bar indicates $10 \mu \mathrm{m}$. 
unobstructed imaging of all cells.

- Free ferrofluid is carried over from the sample preparation in the CellTracks AutoPrep into the final processed sample. When inserted in the magnest, free ferrofluid is also forced to the analysis surface where it arrives before the majority of the cells. At the analysis surface, free ferrofluid particles form aggregates which result in distinctive distortion and reduction of fluorescence signals as is shown in Figure 1.4E. Removal of free ferrofluid in the final sample will increase fluorescence yield and enable accurate morphological characterization of all cells.

\subsection{Thesis OUtLINe}

To improve upon the sensitivity and the ability to quantify antigen expression of the CellTracks Analyzer II, we designed, build and tested an image cytometer, termed CellTracks TDI. Chapter 2 describes the CellTracks TDI system, which uses lasers, a higher magnification objective and a TDI camera to achieve high resolution and high sensitivity images of detected events with minimal overhead time. In chapter 3 the CellTracks TDI was used for the automated identification and quantification of leukocytes and CTC, which could be subdivided into intact, apoptotic and fragmented CTC. Chapter 4 describes the design, development and testing of structures that align and concentrate all cells at the analysis surface of a CellSearch cartridge. The best performing structure was implemented for use with the CellTracks TDI system and this is described in chapter 5. The time required to image all cells on the analysis surface was thereby significantly reduced. Also, the recovery of cells using both a flat analysis surface and using the new microstructures was determined to be linear with an excellent correlation. In chapter 6 the quantitative and qualitative effects of free ferrofluid on imaging of beads, leukocytes and cultured tumor cells in a CellSearch cartridge were evaluated. We determined the maximum concentration of free ferrofluid that is allowed to be in a sample in order to optimize the fluorescence yield and allow for the use of bright-field images to accurately detect the outlines of a cell. Finally, chapter 7 describes an automated system developed and build for the removal of free ferrofluid from blood samples immunomagnetically enriched for CTC to below the maximum allowable ferrofluid concentration for optimal characterization of CTC.

\subsection{REFERENCES}

[1] D. Hanahan and R. Weinberg, "The hallmarks of cancer," Cell, vol. 100, no. 1, pp. 57 $-70,2000$.

[2] M. Sporn, "The war on cancer," The Lancet, vol. 347, no. 9012, pp. 1377 - 1381, 1996. 
[3] T. Asworth, "A case of cancer in which cells similar to those in tumors were seen in the blood after death," Aust Med J, vol. 14, pp. 114-146, 1869.

[4] S. Paget, "The distribution of secondary growths in cancer of the breast," The Lancet, vol. 133, no. 3421, pp. 571-573, 1889.

[5] R. Siegel, D. Naishadham, and A. Jemal, "Cancer statistics, 2012," CA Cancer Journal for Clinicians, vol. 62, no. 1, pp. 10-29, 2012.

[6] P. Went, A. Lugli, S. Meier, M. Bundi, M. Mirlacher, G. Sauter, and S. Dirnhofer, "Frequent epcam protein expression in human carcinomas," Human Pathology, vol. 35, no. 1, pp. 122-128, 2004.

[7] S. Cohen, C. Punt, N. Iannotti, B. Saidman, K. Sabbath, N. Gabrail, J. Picus, M. Morse, E. Mitchell, C. Desch, M. Miller, G. Doyle, H. Tissing, L. Terstappen, and N. Meropol, "The relationship of circulating tumor cells to tumor response, progression-free survival, and overall survival in patients with metastatic colorectal cancer," Journal of Clinical Oncology, vol. 26(19), pp. 3213-3221, 2008.

[8] M. Cristofanilli, T. Budd, M. Ellis, A. Stopeck, J. Matera, M. Miller, J. Reuben, G. Doyle, W. Allard, L. Terstappen, and D. Hayes, "Circulating tumor cells, disease progression, and survival in metastatic breast cancer," $N$ Engl J Med, vol. 351(8), pp. 781-791, 2004 .

[9] D. Hayes, M. Cristofanilli, G. Budd, M. Ellis, A. Stopeck, M. Miller, J. Matera, W. Allard, G. Doyle, and L. Terstappen, "Circulating tumor cells at each follow-up time point during therapy of metastatic breast cancer patients predict progressionfree and overall survival.," Clinical cancer research : an official journal of the American Association for Cancer Research, vol. 12, no. 14 Pt 1, pp. 4218-4224, 2006.

[10] J. De Bono, H. Scher, R. Montgomery, C. Parker, M. Miller, H. Tissing, G. Doyle, L. Terstappen, K. Pienta, and D. Raghavan, "Circulating tumor cells predict survival benefit from treatment in metastatic castration resistant prostate cancer," Clin Can Res, vol. 14(19), pp. 6302-6309, 2008.

[11] V. Hofman, M. Ilie, E. Long, E. Selva, C. Bonnetaud, T. Molina, N. Venissac, J. Mouroux, P. Vielh, and P. Hofman, "Detection of circulating tumor cells as a prognostic factor in patients undergoing radical surgery for non-small-cell lung carcinoma: Comparison of the efficacy of the cellsearch assay and the isolation by size of epithelial tumor cell method," International Journal of Cancer, vol. 129, no. 7, pp. 1651-1660, 2011.

[12] W. Allard, J. Matera, M. Miller, M. Repollet, M. Connelly, C. Rao, A. Tibbe, J. Uhr, and L. Terstappen, "Tumor cells circulate in the peripheral blood of all major carcinomas but not in healthy subjects or patients with nonmalignant diseases," Clinical Cancer Research, vol. 10(20), pp. 6897-6904, 2004.

[13] S. Riethdorf, H. Fritsche, V. Maller, T. Rau, C. Schindlbeck, B. Rack, W. Janni, C. Coith, K. Beck, F. Janicke, S. Jackson, T. Gornet, M. Cristofanilli, and K. Pantel, "Detection of circulating tumor cells in peripheral blood of patients with metastatic breast cancer: A validation study of the cell search system," Clinical Cancer Research, vol. 13, no. 3, pp. 920-928, 2007.

[14] G. Budd, M. Cristofanilli, M. Ellis, A. Stopeck, E. Borden, M. Miller, J. Matera, M. Repollet, G. Doyle, L. Terstappen, and D. Hayes, "Circulating tumor cells versus imaging - predicting overall survival in metastatic breast cancer," Clinical Cancer Research, vol. 12, no. 21, pp. 6403-6409, 2006. 
[15] A. Hartkopf, P. Wagner, D. Wallwiener, T. Fehm, and R. Rothmund, "Changing levels of circulating tumor cells in monitoring chemotherapy response in patients with metastatic breast cancer," Anticancer Research, vol. 31, no. 3, pp. 979-984, 2011.

[16] J. Smerage, D. Hayes, and E. Winer, "Phase III randomized study of treatment decision making based on levels of circulating tumor cells in women with metastatic breast cancer undergoing chemotherapy," -, vol. -, pp. -, 2006-2012.

[17] N. Hynes and D. Stern, "The biology of erbb-2/neu/her-2 and its role in cancer," Biochimica et Biophysica Acta - Reviews on Cancer, vol. 1198, no. 2-3, pp. 165-184, 1994.

[18] D. Slamon, B. Leyland-Jones, S. Shak, H. Fuchs, V. Paton, A. Bajamonde, T. Fleming, W. Eiermann, J. Wolter, M. Pegram, J. Baselga, and L. Norton, "Use of chemotherapy plus a monoclonal antibody against her2 for metastatic breast cancer that overexpresses her2," New England Journal of Medicine, vol. 344, no. 11, pp. 783-792, 2001.

[19] D. Slamon, G. Clark, and S. Wong, "Human breast cancer: Correlation of relapse and survival with amplification of the her-2/neu oncogene," Science, vol. 235, no. 4785, pp. 177-182, 1987.

[20] M. Press, L. Bernstein, P. Thomas, L. Meisner, J. Zhou, Y. Ma, G. Hung, R. Robinson, C. Harris, A. El-Naggar, D. Slamon, R. Phillips, J. Ross, S. Wolman, and K. Flom, "Her-2/neu gene amplification characterized by fluorescence in situ hybridization: Poor prognosis in node-negative breast carcinomas," Journal of Clinical Oncology, vol. 15, no. 8, pp. 2894-2904, 1997.

[21] P. Carter, L. Presta, C. Gorman, J. Ridgway, D. Henner, W. Wong, A. Rowland, C. Kotts, M. Carver, and H. Shepard, "Humanization of an anti-p185(her2) antibody for human cancer therapy," Proceedings of the National Academy of Sciences of the United States of America, vol. 89, no. 10, pp. 4285-4289, 1992.

[22] J. Baselga, L. Norton, J. Albanell, Y. Kim, and J. Mendelsohn, "Recombinant humanized anti-her2 antibody (herceptin $(\mathrm{tm})$ ) enhances the antitumor activity of paclitaxel and doxorubicin against her2/neu overexpressing human breast cancer xenografts," Cancer Research, vol. 58, no. 13, pp. 2825-2831, 1998.

[23] G. Attard, A. Reid, T. Yap, F. Raynaud, M. Dowsett, S. Settatree, M. Barrett, C. Parker, V. Martins, E. Folkerd, J. Clark, C. Cooper, S. Kaye, D. Dearnaley, G. Lee, and J. De Bono, "Phase I clinical trial of a selective inhibitor of cyp17, abiraterone acetate, confirms that castration-resistant prostate cancer commonly remains hormone driven," Journal of Clinical Oncology, vol. 26(28), no. 28, pp. 4563$4571,2008$.

[24] A. Reid, G. Attard, D. Danila, N. Oommen, D. Olmos, P. Fong, L. Molife, J. Hunt, C. Messiou, C. Parker, D. Dearnaley, J. Swennenhuis, L. Terstappen, G. Lee, T. Kheoh, A. Molina, C. Ryan, E. Small, H. Scher, and J. De Bono, "Significant and sustained antitumor activity in post-docetaxel, castration-resistant prostate cancer with the cyp17 inhibitor abiraterone acetate," Journal of Clinical Oncology, vol. 28, no. 9, pp. 1489-1495, 2010.

[25] G. Attard, J. Swennenhuis, D. Olmos, A. Reid, E. Vickers, R. Hern, R. Levink, F. Coumans, J. Moreira, R. Riisnaes, N. Oomen, G. Hawche, C. Jameson, E. Thompson, R. Sipkema, C. Carden, C. Parker, D. Dearnaley, S. Kaye, C. Cooper, A. Molina, M. Cox, L. Terstappen, and J. de Bono, "Characterization of erg, ar and pten status in circulating tumor cells from patients with castration-resistant prostate cancer," Cancer Research, vol. 69, pp. 2912-2918, 2009. 
[26] R. Moll, W. Franke, and D. Schiller, "The catalog of human cytokeratins: Patterns of expression in normal epithelia, tumors and cultured cells," Cell, vol. 31, no. 1, pp. 11-24, 1982.

[27] S. Mani, W. Guo, M. Liao, E. Eaton, A. Ayyanan, A. Zhou, M. Brooks, F. Reinhard, C. Zhang, M. Shipitsin, L. Campbell, K. Polyak, C. Brisken, J. Yang, and R. Weinberg, "The epithelial-mesenchymal transition generates cells with properties of stem cells," Cell, vol. 133, no. 4, pp. 704-715, 2008.

[28] G. Vona, A. Sabile, M. Louha, V. Sitruk, S. Romana, K. Schutze, F. Capron, D. Franco, M. Pazzagli, M. Vekemans, B. Lacour, C. Brechot, and P. PaterliniBrechot, "Isolation by size of epithelial tumor cells: A new method for the immunomorphological and molecular characterization of circulating tumor cells," $\mathrm{Am}$ J Pathol, vol. 156(1), pp. 57-63, 2000.

[29] P. Pinzani, B. Salvadori, L. Simi, S. Bianchi, V. Distante, L. Cataliotti, M. Pazzagli, and C. Orlando, "Isolation by size of epithelial tumor cells in peripheral blood of patients with breast cancer: correlation with real-time reverse transcriptasepolymerase chain reaction results and feasibility of molecular analysis by laser microdissection," Human Pathology, vol. 37, no. 6, pp. 711-718, 2006.

[30] F. Coumans, G. van Dalum, M. Beck, and L. Terstappen, "Filter requirements for circulating tumor cell enrichment and detection," Submitted for publication, -.

[31] S. Nagrath, L. Sequist, S. Maheswaran, D. Bell, D. Irimia, L. Ulkus, M. Smith, E. Kwak, S. Digumarthy, A. Muzikansky, P. Ryan, U. Balis, R. Tompkins, D. Haber, and M. Toner, "Isolation of rare circulating tumour cells in cancer patients by microchip technology," Nature, vol. 450, pp. 1235-1241, 2007.

[32] S. Stott, C. Hsu, D. Tsukrov, M. Yu, D. Miyamoto, B. Waltman, M. Rothenberg, A. Shah, M. Smas, G. Korir, F. Floyd Jr, A. Gilman, J. Lord, D. Winokur, S. Springer, D. Irimia, S. Nagrath, L. Sequist, R. Lee, K. Isselbacher, S. Maheswaran, D. Haber, and M. Toner, "Isolation of circulating tumor cells using a microvortex-generating herringbone-chip," Proceedings of the National Academy of Sciences of the United States of America, vol. 107, no. 43, pp. 18392-18397, 2010.

[33] R. Krivacic, A. Ladanyi, D. Curry, H. Hsieh, P. Kuhn, D. Bergsrud, J. Kepros, T. Barbera, M. Ho, L. Chen, R. Lerner, and R. Bruce, "A rare-cell detector for cancer," PNAS, vol. 101, pp. 10501-10504, 2004.

[34] H. Hsieh, D. Marrinucci, K. Bethel, D. Curry, M. Humphrey, R. Krivacic, J. Kroener, L. Kroener, A. Ladanyi, N. Lazarus, P. Kuhn, R. Bruce, and N. J, "High speed detection of circulating tumor cells," Biosens Bioelectron, vol. 21, pp. 1893-1899, 2006 .

[35] K. Pachmann, P. Heiss, U. Demel, and G. Tilz, "Detection and quantification of small numbers of circulating tumour cells in peripheral blood using laser scanning cytometer," Clinical Chemistry and Laboratory Medicine, vol. 39, no. 9, pp. 811-817, 2001.

[36] K. Pachmann, J. Clement, C. Schneider, B. Willen, O. Camara, U. Pachmann, and K. Hoffken, "Standardized quantification of circulating peripheral tumor cells from lung and breast cancer," Clinical Chemistry and Laboratory Medicine, vol. 43, no. 6, pp. 617-627, 2005.

[37] K. Pachmann, O. Camara, A. Kavallaris, S. Krauspe, N. Malarski, M. Gajda, T. Kroll, C. Jörke, U. Hammer, A. Altendorf-Hofmann, C. Rabenstein, U. Pachmann, I. Runnebaum, and K. Höffken, "Monitoring the response of circulating epithelial tumor cells to adjuvant chemotherapy in breast cancer allows detection of patients at risk of early relapse," JCO, vol. 26, pp. 1208-1215, 2008. 
[38] A. Tibbe, B. de Grooth, J. Greve, G. Dolan, C. Rao, and L. Terstappen, "Magnetic field design for selecting and aligning immunomagnetic labeled cells," Cytometry, vol. 47(3), pp. 163-172, 2002.

[39] J. Kraan, S. Sleijfer, M. Strijbos, M. Ignatiadis, D. Peeters, J. Pierga, F. Farace, S. Riethdorf, T. Fehm, L. Zorzino, A. Tibbe, M. Maestro, R. Gisbert-Criado, G. Denton, J. De Bono, C. Dive, J. Foekens, and J. Gratama, "External quality assurance of circulating tumor cell enumeration using the CellSearch system: A feasibility study," Cytometry Part B - Clinical Cytometry, vol. 80B, no. 2, pp. 112118, 2011.

[40] A. Tibbe, C. Miller, and L. Terstappen, "Statistical considerations for enumeration of circulating tumor cells," Cytometry Part A, vol. 71A, pp. 154-162, 2007.

[41] F. Coumans, C. Doggen, G. Attard, J. de Bono, and L. Terstappen, "All circulating $\mathrm{EpCAM}+\mathrm{CD} 45-\mathrm{CK}+$ but not EpCAM $+\mathrm{CD} 45+\mathrm{CK}+$ objects predict overall survival in castration-resistant prostate cancer," Annals of Oncology, vol. 21(9), pp. 18511857, 2010.

[42] A. Tibbe, B. de Grooth, J. Greve, C. Rao, G. Dolan, and L. Terstappen, "Cell analysis system based on compact disk technology," Cytometry, vol. 47(3), pp. 173182,2002 . 


\section{CellTRACKS TDI - AN IMAGE Cytometer fOR CEll CHARACTERIZATION}

Characterization of rare cells usually requires high sensitivity quantification of multiple parameters. Detection of morphological features of these cells is highly desired when routinely identifying circulating tumor cells (CTC) in blood of patients. We have designed an image cytometer intended for fast and sensitive routine analysis of CTC.

The image cytometer features: 375, 491 and $639 \mathrm{~nm}$ laser lines shaped into a square homogeneous illumination area, a $40 \times / 0.6 \mathrm{NA}$ objective and a piëzo microscope objective positioner to move the objective. Continuous signal acquisition is made possible by using a CCD camera operating in TDI mode synchronized to the movement of two servo scan stages that move the sample. Image J is used for dedicated image analysis.

The limit of fluorescence sensitivity is $120 \mathrm{PE}$ molecules on a bead with a diameter of $6.8 \mu \mathrm{m}$, at a scanning speed of $1.0 \mathrm{~mm} / \mathrm{s}$. The resolution of the imaging system is $0.76 \mu \mathrm{m}$ in the TDI scan direction at a wavelength of $580 \mathrm{~nm}$. Identification of cells is facilitated by scatter plots of the fluorescent parameters in which each individual event can be viewed for its morphological features by fluorescence as well as bright-field.

The image cytometer measures quantitative fluorescence and morphological features at a high sensitivity, high resolution and with minimal overhead time. It has the ability to relocate events of interest for further detailed analysis. The system can be used for routine identification and characterization of rare cells.

This chapter has been published in Cytometry A, 2011 doi: 10.1002/cyto.a.21024 


\subsection{INTRODUCTION}

Flow cytometry (FCM) is the standard technology for characterization and enumeration of cells in a heterogeneous cell mixture. It is routinely used for diagnosis and monitoring of diseases that result in specific changes of a particular cell population. Yet, for characterization of rare populations FCM has its limitations. This explains the emergence of alternative cell analysis technologies for dedicated applications [1-4].

We aim to routinely analyze and enumerate tumor cells that circulate at extremely low concentrations in the blood of cancer patients. We started by using FCM as the analysis platform [5-7]. The variable background of the events that classified as tumor cells in a multidimensional gate in FCM [5-7], observed in blood of normal donors urged us to use morphological confirmation. Fluorescence microscopy in combination with immunomagnetic enrichment of circulating tumor cells (CTC) proved to be the right combination. It removes the background and identifies the CTC on basis of their fluorescence signature and morphological features such as round to oval shape, a diameter greater than $4 \mu \mathrm{m}$ and an intact nucleus that is surrounded by the cytoplasm. This combination formed the basis for Veridex's CellTracks ${ }^{\circledR}$ System. Using this system, the clinical relevance of CTC was demonstrated in prospective multicenter clinical trials. Metastatic breast and colorectal patients with 5 or more CTC and mestastatic prostrate patients with 3 or more CTC in $7.5 \mathrm{ml}$ of blood have a significant worse prognosis as compared to patients that have less than 3 or 5 CTC [8-10]. Currently the CellTracks ${ }^{\circledR}$ Analyzer II ${ }^{\circledR}$ System (Analyzer II) is the only IVD 510(k) system used for serial monitoring of CTC's in metastatic breast (MBC), colorectal (MCRC) and prostate (MPC) patients.

The sensitivity of detection and ability to quantify antigen expression of the Analyzer II System is, however, not as good as in FCM. This is caused by trade-offs between spatial resolution, speed of data collection, storage requirements, image analysis, and the wish to keep the total analysis time acceptable for routine applications. Here we describe the development and characterization of CellTracks TDI, an improved image cytometer for potential future use in CTC analysis.

\subsection{Materials And Methods}

\subsubsection{BEADS}

The sensitivity of the CellTracks TDI was tested with $6.8 \mu \mathrm{m}$ Quantibrite beads (Becton Dickinson, Franklin Lakes, NJ, USA). The 4 bead populations contained respectively 515, 5956, 26653 and 69045 Phycoerythrin (PE) molecules, with CVs of $14.3 \%, 12.1 \%, 14.3 \%$ and $13.3 \%$ respectively according to the manufacturer. 
The linearity and the variation of the CellTracks TDI were determined using Rainbow Linear Particles, RLP-30-5 (Spherotech Inc., Libertyville, IL, USA). The sample contains a mixture of rainbow particles with five different fluorescent intensities. The intensity drops by factors of 2 from the brightest particle. The particles have a CV of $2.0 \%$. This small CV makes them useful to determine the $\mathrm{CV}$ increase introduced by the measurement system. The size of the particles ranges from 3.0 to $3.4 \mu \mathrm{m}$.

One tube of Quantibrite beads was reconstituted using $0.5 \mathrm{ml} 1 \times$ PBS. After reconstitution, $360 \mu$ of the Quantibrite PE sample was transferred to a standard CellTracks cartridge and placed inside a CellTracks Magnest. The assembly was left inverted for 3 hours, allowing the beads to sediment to the analysis surface by gravity. This procedure is necessary because these beads are not magnetic and the Magnest only presents magnetic objects at the analysis surface of the cartridge. This resulted in around 50 beads per $\mathrm{mm}^{2}$ that were adhered to the upper surface of the cartridge. Observation under a microscope showed that the majority of the beads remained at the imaging surface, after turning the assembly right side up, even under the influence of gravity. The Quantibrite PE beads could now be measured under conditions similar to a CTC sample.

Rainbow linear particles were diluted in $1 \times$ PBS and deposited on a glass slide and sealed with a cover slip. The cover slip was fixed to the glass slide using nail polish to prevent dehydration of the sample.

\subsubsection{CTC POSITIVE SAMPLES}

Blood samples analyzed using the CellTracks System (Veridex LLC, Raritan, NJ, USA) that contained CTC were reanalyzed using CellTracks TDI. In brief, $7.5 \mathrm{ml}$ of blood was magnetically enriched using ferrofluids targeting the epithelial cell adhesion molecule (EpCAM) and labeled with DAPI, Cytokeratins 8, 18 and $19 \mathrm{PE}$ and CD45-APC using the CellTracks AutoPrep ${ }^{\circledR}$ System (Veridex LLC). The $360 \mu$ lenriched sample was then transferred into the cartridge and placed in the Magnest and analyzed on the Analyzer II. The final sample, as prepared by the AutoPrep System, contains 85\% of the CTC in the original sample together with a small percentage of leukocytes and some debris. These cartridges were then reanalyzed in the CellTracks TDI system.

\subsection{InStrumentation}

\subsubsection{OPTICAL SYSTEM}

A schematic representation of the optical layout of the CellTracks TDI image cytometer is given in Figure 2.1.

Three lasers are available for excitation, which may be used separately or in any combination. Emission band pass filters (520/35, 585/40 and 670/30; 


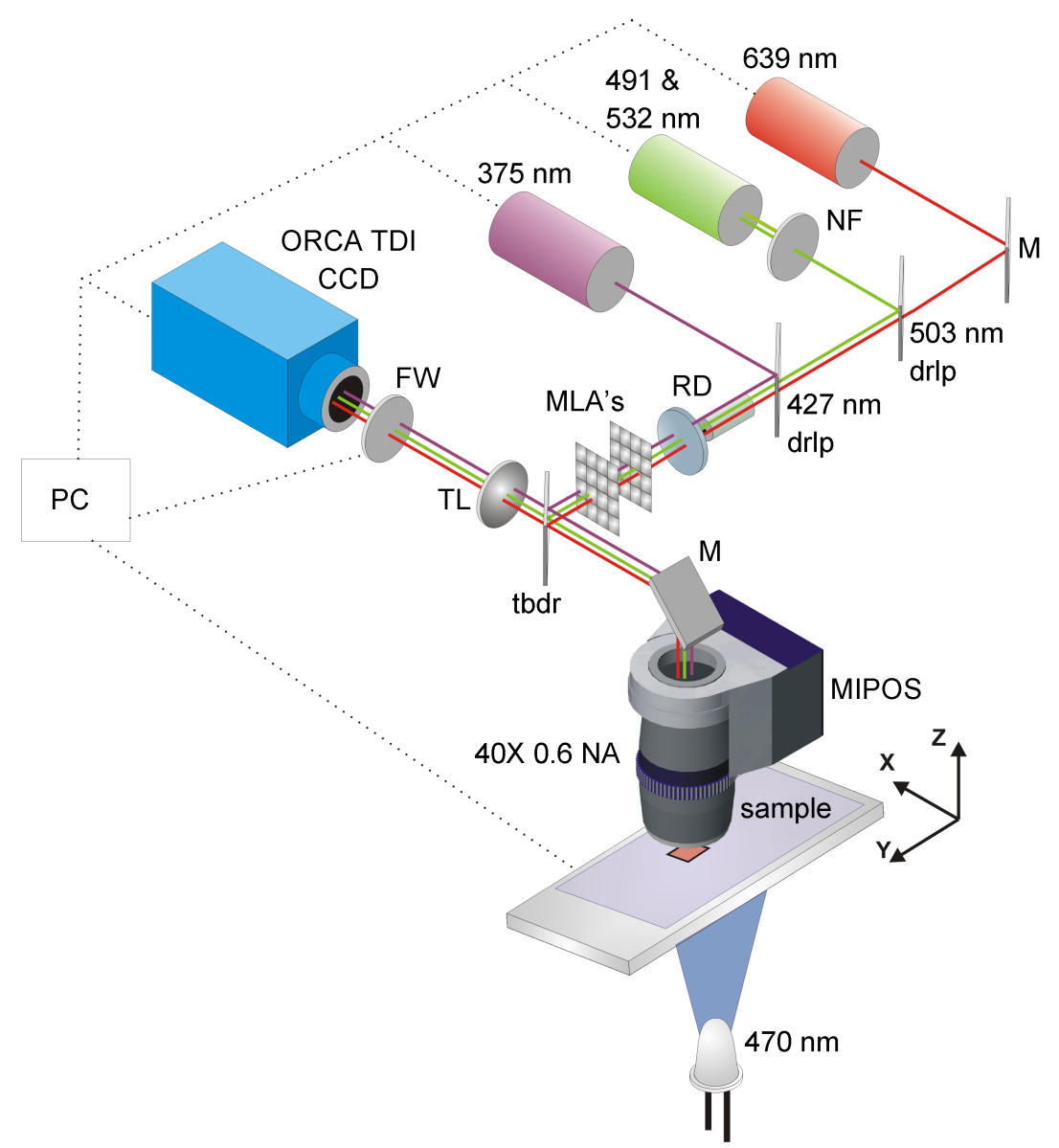

Figure 2.1 : Optical layout of the CellTracks TDI system. A 375 nm, 491 \& 532 $\mathrm{nm}$ and $639 \mathrm{~nm}$ laser, $\mathrm{M}=$ mirror, drlp = dichroic longpass filter, $\mathrm{RD}=$ rotating diffuser, MLA = micro-lens array, tbdr = triple-band dichroic, MIPOS $=$ piëzo actuated microscope objective positioning system, TL $=$ tube lens, $\mathrm{FW}=$ filter wheel, $470 \mathrm{~nm}=$ wavelength of bright-field LED underneath sample. 
Semrock, Rochester, NY, USA) are used to eliminate unwanted emission. They are contained in the filter wheel (FW). To merge the beams into 1 parallel overlapping beam, we use multiple dichroic filters (Semrock). The output of the $16 \mathrm{~mW} 375 \mathrm{~nm}$ solid-state laser (Power Technology, Alexander, AR, USA) is coupled in using a $427 \mathrm{~nm}$ dichroic long pass filter (drlp). From the dual line laser (491 nm and $532 \mathrm{~nm}$, Cobolt AB, Solna, Sweden) only the $20 \mathrm{~mW} 491 \mathrm{~nm}$ output is coupled in using a $503 \mathrm{~nm}$ drlp. The $532 \mathrm{~nm}$ laserline is filtered out using a $532 \mathrm{~nm}$ notch filter (NF / Semrock) because it is not reflected by the triple-band dichroic that is described later and used to direct the laser beams into the objective. The third beam comes from a $30 \mathrm{~mW}, 639 \mathrm{~nm}$ laser (Power Technology, USA). It is reflected by a mirror and passes through the $427 \mathrm{~nm}$ and the $503 \mathrm{~nm}$ drlp.

The coherence length of the laser lines is much larger than the dimensions of the set-up. For example, the $491 \mathrm{~nm}$ line has a bandwidth of $30 \mathrm{MHz}$, hence a coherence length of $3.2 \mathrm{~m}$. To reduce the coherence length, and prevent unwanted interference effects, we use a small motor to rotate the transparent diffuser [11] at $6000 \mathrm{rpm}$. (RD / Suss-MicroOptics, Neuchâtel, Switzerland). It has a pebble-like relief with varying dimensions on its surface, with average pebble dimensions of $40 \times 40 \mu^{2}$. All beams are overlaid at the point where they enter the beam homogenizing optics (Suss-MicroOptics), that create a square homogeneous illumination profile [12-16]. The operation of the homogenizer is explained below. After passing the beam homogenizer, the laser light is redirected by a triple-band dichroic filter (tbdr) that reflects the 375, 491 and $639 \mathrm{~nm}$ laser lines onto the entrance aperture of the $40 \times / 0.6$ NA CFI Plan Fluor ELWD infinity corrected microscope objective (Nikon, Melville, NY, USA). These 3 wavelengths were chosen to be able to excite the DAPI, PE and APC fluorophores that are used in Veridex's CellSearch ${ }^{\circledR}$ technology. The objective can be moved vertically over a range of $400 \mu \mathrm{m}$ using a piëzo positioner (MIPOS 500, Piezosystem Jena, Jena, Germany). The objective then focuses the 3 laser beams on the sample in approximately square illumination spots of $217 \times 217 \mu \mathrm{m}^{2}$ full width half maximum (FWHM), of which the center $180 \times 180 \mu \mathrm{m}^{2}$ are used during imaging of the sample. The resulting (maximum) irradiance at the object plane is $10.8 \mathrm{~W} / \mathrm{cm}^{2}$ at $375 \mathrm{~nm} ; 24.0 \mathrm{~W} / \mathrm{cm}^{2}$ at $491 \mathrm{~nm}$ and $33.6 \mathrm{~W} / \mathrm{cm}^{2}$ at $639 \mathrm{~nm}$.

Part of the emitted fluorescence is collected by the objective. It passes through the triple band dichroic filter. A motorized filter wheel (Thorlabs, Newton, NJ, USA) selects the correct emission filter for each particular fluorescent probe. The emission light is focused by a $160 \mathrm{~mm}$ achromatic tube-lens (TL / Linos Photonics, Goëttingen, Germany) onto the high sensitivity 12-bit Peltier cooled ORCA C4742-95-12ERT TDI camera (Hamamatsu, Hamamatsu City, Japan) with $1344 \times 1024$ pixels of $6.45 \times 6.45 \mu^{2}$. This camera can both operate in TDI and frame transfer mode. A blue 470 nm LED (Philips-Lumileds, San Jose, CA, USA) underneath the sample is used for bright-field illumination. 


\subsubsection{BEAM HOMOGENIZER}

When scanning the sample at a typical speed of $1 \mathrm{~mm} / \mathrm{s}$, each pixel in the object space $(0.2 \mu \mathrm{m} \times 0.2 \mu \mathrm{m})$ is illuminated during $200 \mu \mathrm{s}$. The laser beam has a diameter of $700 \mu \mathrm{m}$ and hits the diffuser at $10 \mathrm{~mm}$ from its center. As the diffuser rotates at $6000 \mathrm{rpm}$, the beam travels in $200 \mu$ s over $1256 \mu \mathrm{m}$ of the diffuser surface, which is 1.8 times the beam diameter and 31 times the average pebble size on the diffuser. This creates sufficient randomization of the phase of the beam during the time a pixel is illuminated and thereby greatly reduces interference effects due to coherence of the laser beam.

The beam homogenizer uses two square micro-lens arrays that consist of a periodic structure of micro-lenses, with pitch $\mathrm{P}_{\text {MLA }}$ (Figure 2.2, Panel A). The first array $\left(\mathrm{MLA}_{1}\right)$ focuses the beam on the second array $\left(\mathrm{MLA}_{2}\right)$, resulting in multiple point sources. The light from all these separate sources is collected by the microscope objective and overlaid in the focal plane where they form a quadratic illumination profile with a homogeneous intensity (flat top profile) with a size given by Equation (2.1) [17]:

$$
D_{F T}=\frac{P_{M L A} \cdot f_{O B J}}{f_{1} \cdot f_{2}}\left(\left(f_{1}+f_{2}\right)-a_{12}\right)
$$

Where, $\mathrm{P}_{\mathrm{MLA}}=0.50 \mathrm{~mm}, \mathrm{f}_{\mathrm{obj}}=5 \mathrm{~mm}$, the focal length of the microscope objective, $\mathrm{f}_{1}=\mathrm{f}_{2}=15.54 \mathrm{~mm}$, the focal lengths of the micro-lens arrays and $\mathrm{a}_{12}=15.54 \mathrm{~mm}$, the distance between the two micro-lens arrays.

When all components are positioned according to the above described distances, the resulting illumination profile is approximately square with a FWHM of $161 \mu \mathrm{m}$. However, the actual width of the illumination profile, with a CV of $<5 \%$, is then about $130 \mu \mathrm{m}$. This results in the need to scan 21 'strips' to cover an entire sample cartridge. To reduce this number and still be able to see the edges of the illumination profile for focus determination purposes, $a_{12}$ may be slightly increased. To avoid large aspect ratios of the images and reduce the number of events that are located at the border of an image, the images are stitched together in groups of four. The accuracy of overlay of fluorescent channels is limited by the stage accuracy, which is $0.2 \mu \mathrm{m}$.

\subsubsection{FEED FORWARD FOCUSING}

The analysis surface is never perfectly flat, resulting in a variation in focus position in the $\mathrm{Z}$ direction when scanning the surface. Prior to a scan, the focus positions are determined automatically at a grid of 6 by 5 positions on the surface. An automatic focusing algorithm uses the reflection of the red laser profile from the glass-sample interface. It integrates the reflection over the $400 \mu \mathrm{m}$ range of the piëzo positioner using the TDI camera. This generates an image that contains the profile of the illumination spot for every part of the $400 \mu \mathrm{m}$ range. A custom made algorithm is then used 
A

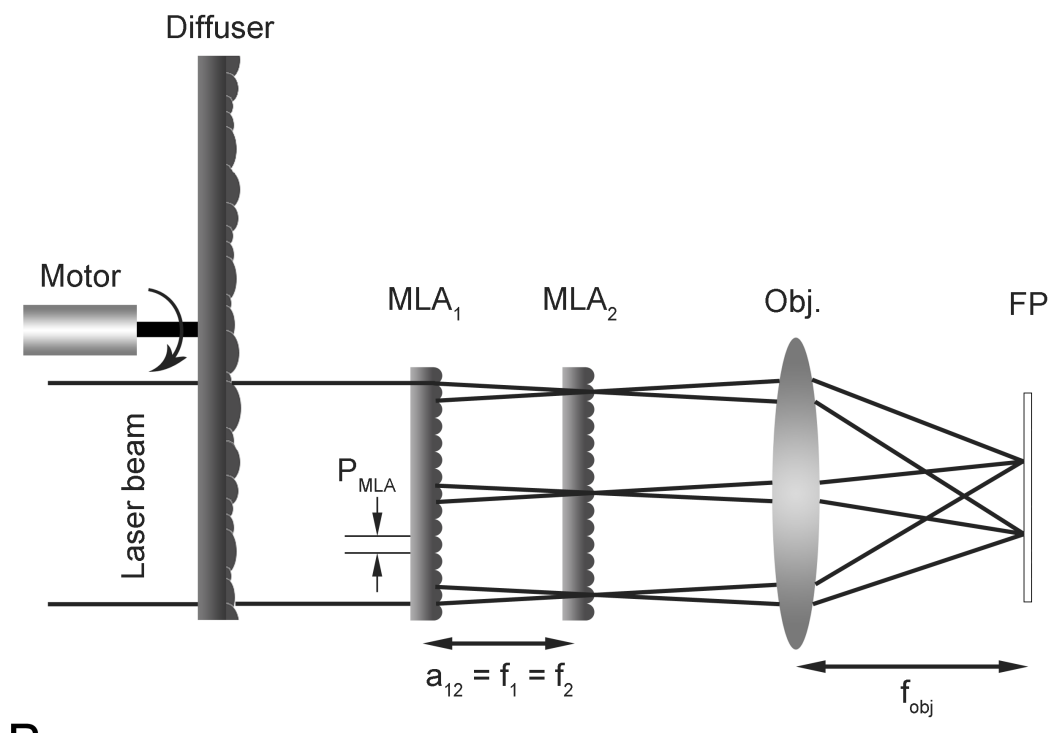

B

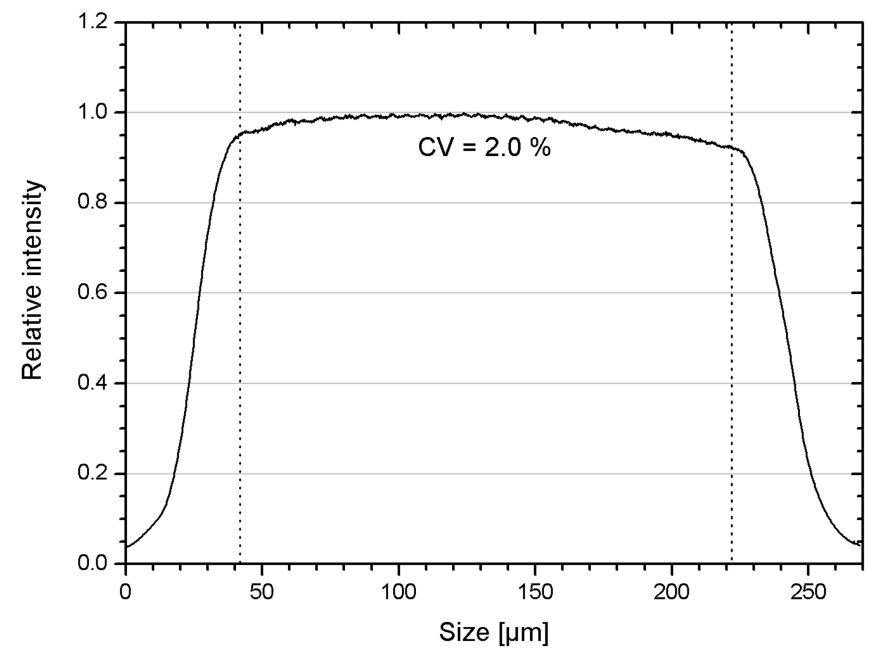

FIgURE 2.2: (A) Schematic representation (not to scale) of the beam homogenizing optics. $\mathrm{P}_{\mathrm{MLA}}=$ pitch of the micro-lens arrays, MLA 1 and $\mathrm{MLA}_{2}=$ micro-lens arrays, $\mathrm{FP}=$ focal plane, $\mathrm{Obj}=$ objective, $\mathrm{a}_{12}=$ distance between $\mathrm{MLA}_{1}$ and $\mathrm{MLA}_{2}$ and $\mathrm{f}_{1}, \mathrm{f}_{2}$ and $\mathrm{f}_{\mathrm{obj}}=$ focal lengths of $\mathrm{MLA}_{1}, \mathrm{MLA}_{2}$ and objective respectively. (B) Illumination profile determined by imaging the emission of a thin layer of PE upon $491 \mathrm{~nm}$ laser excitation. The dotted lines indicate the $180 \mu \mathrm{m}$ width of the illumination area that is used during scanning. 
to find the position were the illumination profile has the sharpest defined edges, indicating the focus position. These positions are then fitted by a $3 \mathrm{D}$ spline that estimates the correct focus position with an accuracy of $0.5 \mu \mathrm{m}$ (smaller than the depth of field of the microscope objective, which is $1.5 \mu \mathrm{m}$ for light with a wavelength of $550 \mathrm{~nm}$ ) at each point on the surface. During a scan, the 3D spline fit is used to focus the objective with the MIPOS operating in feed forward mode. The bandwidth of the piëzo system was determined by measuring the response at increasing frequencies. The $-3 \mathrm{~dB}$ point was found to be at $4.5 \mathrm{~Hz}$, resulting in a 'bandwidth' of $1.8 \mathrm{~mm} / \mathrm{sec}$. At a default scan speed of $1 \mathrm{~mm} / \mathrm{sec}$, this translates to $1.8 \mathrm{~mm} / \mathrm{mm}$, which is more than enough to follow the variations in height of a typical cartridge, which are in the order of $5 \mu \mathrm{m} / \mathrm{mm}$.

\subsubsection{SAMPLE SCANNING}

The cartridge is scanned through the $180 \times 180 \mu \mathrm{m}^{2}$ square illumination profile, see Figure 2.3, Panel A. It is moved in the $\mathrm{X}$ and $\mathrm{Y}$ direction by two stacked servo stages, featuring brushless DC motors, (M-605.2DD from Physik Instrumente, Karlsruhe, Germany) with a maximum continuous scan speed of $50 \mathrm{~mm} / \mathrm{s}$ and a travel range of $50 \mathrm{~mm}$, resulting in a total area of $2500 \mathrm{~mm}^{2}$ that can be covered. The complete analysis surface is scanned in the $\mathrm{Y}$ direction in multiple adjacent strips with a width of $180 \mu \mathrm{m}$. At the end of each strip, the cartridge is moved $180 \mu \mathrm{m}$ in the $\mathrm{X}$ direction and to the start of a new line in the Y direction. Then, the next strip is scanned. Precautions are taken to prevent effects due to backlash of the scan system. Each strip scan starts by first moving the slide to a fixed position where the beam is outside the area to be scanned. Next, the scan is started and data are recorded not sooner than after passing a second fixed position on the surface. This procedure is followed to ensure that the stage has achieved its final speed when data are recorded. The overhead time introduced by the acceleration and deceleration of the stages is about $1 \mathrm{sec}$ per strip. Two built-in encoders with a resolution of $0.1 \mu \mathrm{m}$ continuously determine the actual position of the stages.

\subsubsection{TDI CAMERA}

The CCD camera operates in TDI mode, resulting in a continuous readout of collected charges. This mode is illustrated in Figure 2.3, Panel B. When an event is scanned through the illumination profile, the CCD pixels are continuously collecting charges. The collected charges of each pixel row are transferred in parallel one row down to the next pixel row when an external line trigger is received. This trigger is derived from the encoder that reports the actual stage position. The parallel charge shift rate in the CCD is synchronized with the continuous motion of the stages. When the transferred charges reach the last row of CCD pixels, the total collected charge for 


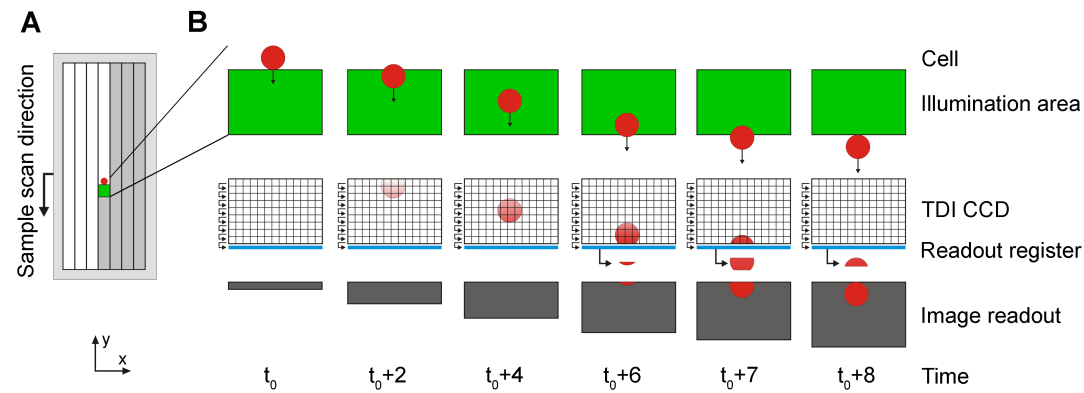

FIGURE 2.3 : Schematic representation of TDI scanning, green = illumination area, red $=$ cell, blue $=$ readout register. (A) The sample is scanned in continuous motion through the stationary illumination area. Light gray strips $=$ scanned area, white strips $=$ area yet to be scanned. (B) Time-steps showing the CCD operating in TDI mode; the charge is accumulated and transferred from row to row at the same speed as the image of the object moves across the CCD surface. The total accumulated charge is finally read out by the readout register.

each pixel is moved to the amplifier and A/D converter. The charge of each separate pixel stems from the integrated light intensity emitted by one corresponding pixel in the object space while the event moves through the illumination profile. As the object moves, the accumulated charges move in the corresponding direction on the CCD, with the same average relative velocity. This makes light integration and CCD readout a continuous process preventing dead time. The resulting image of a strip is 900 pixels wide. Its length is only limited by available memory on the frame grabber, which is $16 \mathrm{MB}$. In order to obtain workable images for analysis in ImageJ and to fit inside the frame grabber buffer, we limit the number of pixels that is read-out into 1 image in the scan direction to 10000, amounting to a total size of 12.9 MB. Each image is then transferred to a larger reserved buffer in RAM memory, from where it is written to disk.

\subsubsection{DISCRETE FINAL MAGNIFICATION}

To prevent blurring and obtain sharp images, precise alignment of the direction of stage movement and the direction of the columns on the CCD is essential. Also, the average speed of the moving object should be matched with the line transfer rate of the camera. Although it is possible to synchronize the camera with a function generator that matches the average scan speed, optimal resolution is only obtained when applying the encoder signal of the translation stages. Small variations in scan speed can then be synchronized with the camera line transfer rate for each position. As the encoder has a resolution of $0.1 \mu \mathrm{m}$, the choice for the magnification, $\mathrm{M}$, is then limited to discrete values given by Equation (2.2): 


$$
M=\frac{\delta p}{\delta e \cdot k}, k=1,2,3,4,5
$$

Where $\delta \mathrm{p}$ is the size of the pixels on the camera and $\delta$ e the encoder resolution. With the use of a counter the required object pixel resolution can be obtained as a multiple of the encoder resolution. In our setup a sampling of $\delta_{\mathrm{s}}=0.2 \mu \mathrm{m} /$ pixel is used requiring a magnification of $32.25 \times$.

\subsubsection{INSTRUMENT CONTROL}

To operate the CellTracks TDI, all different parts have to function in the proper manner at the correct time. To this end, a dedicated software package was written in LabView (National Instruments Corporation, Austin, TX, USA), a graphically oriented programming language. LabView is very appropriate for control of the cytometer as it has many mathematical subroutines and supports an extensive amount of instrument drivers for hardware control. The program can easily be adjusted to accommodate new hardware components. Flowcharts of the dedicated software program are given elsewhere [18].

\subsubsection{IMAGE ANALYSIS}

A scan of a cartridge sized imaging surface $\left(29.7 \times 2.7 \mathrm{~mm}^{2}\right)$ at $0.2 \mu \mathrm{m}$ lateral resolution in 3 fluorescence and 1 bright-field channel generates 16 GB of raw 12-bit image data. This data needs to be processed efficiently to remove the $>99 \%$ of imaged surface area that doesn't contain an event. To this end, we developed a dedicated image analysis routine using ImageJ, a public domain Java program, originally developed at NIH $[19,20]$.

The routine corrects for inhomogeneous illumination, removes the background and selects the events of interest from the raw data. The selected events are then combined and further analyzed to obtain quantitative parameters.

The image analysis procedure is divided in 3 distinct steps. First, the raw $900 \times 10000$ pixel images that have been recorded during a scan are corrected for the variations in the normalized (to the average intensity) illumination profile [21-23] in the direction perpendicular to the scan direction. No correction is needed in the scan direction as the TDI camera averages these intensity variations. Then the background is subtracted by applying the rolling ball method [24] using a diameter of $80 \mu \mathrm{m}$. This diameter is about 3 times larger than the maximum diameter of the future positive events (CTC). This was chosen to ensure that no signal is removed during this step, even when two large events $(30 \mu \mathrm{m})$ are lying side by side.

Second, either the fluorescence or the bright-field image is used to select events of interest, defined as those events that have a pixel intensity above an appropriately chosen fixed threshold, e.g. 50. The fluorescence- and 


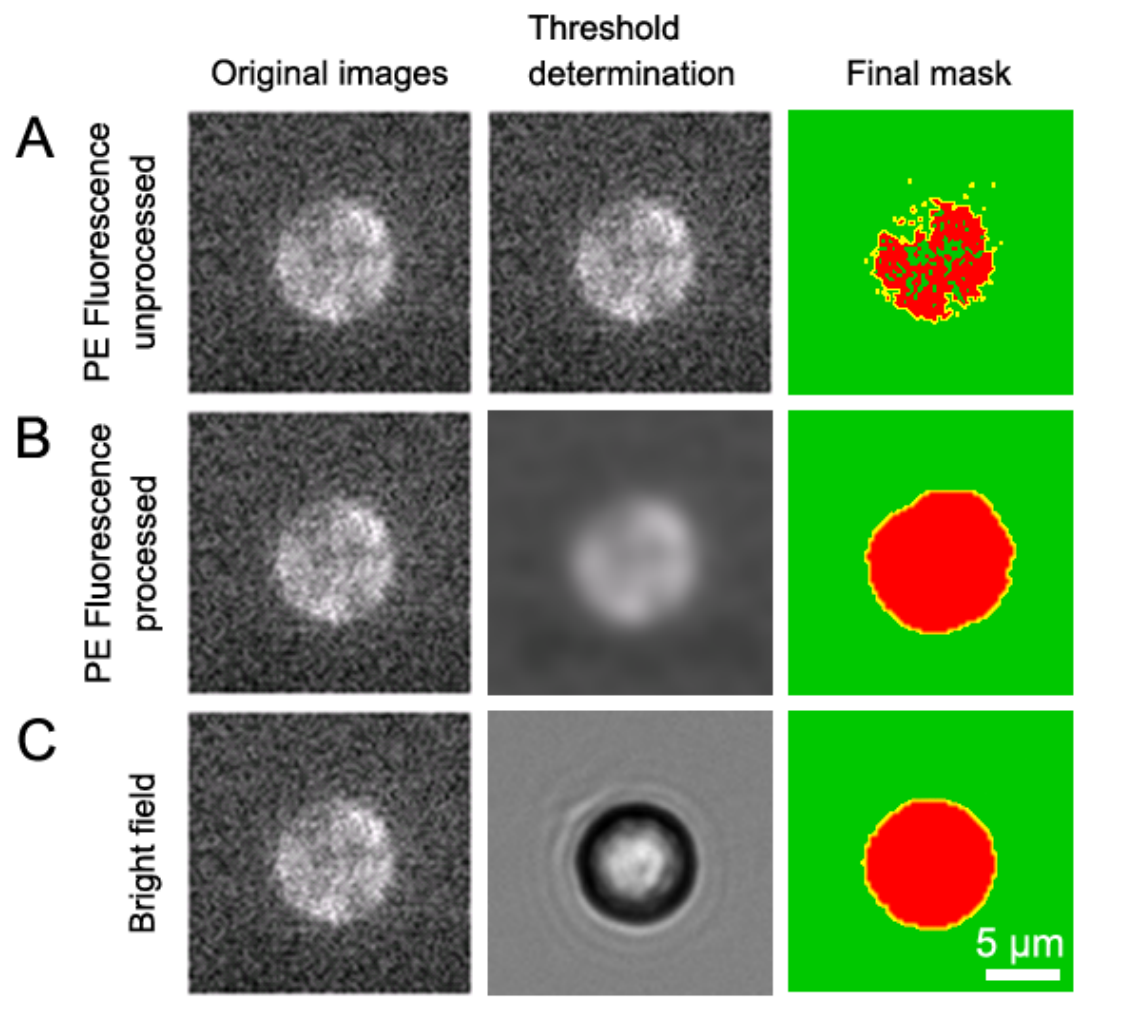

FIGURE 2.4 : Three different ways of thresholding a dim Quantibrite-PE bead with 515 PE molecules. The columns show the original image, method for threshold determination and resulting threshold respectively. Green area = pixel value is below threshold, red area $=$ pixel value above threshold and yellow line $=$ border of the detected object. (A) A simple threshold algorithm was applied and as a result, several pixels inside the bead are not included in the mask. (B) A correct definition of the bead is obtained when the fluorescence image is preprocessed using a Gaussian blur filter with a radius of 5 pixels. (C) Results of using the brightfield channel to determine the final mask. Scale bar represents $5 \mu \mathrm{m}$.

bright-field image data of all events of interest are then combined in one multipage tiff file. This second step drastically reduces the amount of data that needs to be saved, typically by a factor of 100 .

As shown in Figure 2.4, for a Quantibrite bead, some pixels in the background may have an intensity above a threshold level of 3 times $\sigma_{\mathrm{p}}$ (average pixel noise) and some pixels within the bead have an intensity below this threshold.

Using this criterion for threshold determination results in incomplete selection of the bead. This can be prevented by applying a Gaussian blur filter that averages the pixel intensity within a specified radius for each 
pixel. However, it reduces spatial resolution, but averages out the noise thus enabling to segment the particles at a lower threshold limit and obtain a proper mask for the event. When a bright-field image of an event is used to create a mask, the detection limit is independent of the fluorescence level of the event in a different channel. This enables analysis of events with a very dim fluorescence signal.

Third, the last image analysis step creates a mask that defines, for each separate event, its boundaries in all channels. To this end, the fluorescence images of all events are thresholded by the histogram based Otsu algorithm [25]. It determines a threshold by maximizing the between-class variance of pixel intensity in an assumed bimodal histogram and is relatively fast because only the histogram is needed. In the resulting binary image the holes are filled. Then it is used as a mask for the original image, to define the edges.

When a mask has been defined for each image of each event, quantitative and morphological parameters, such as size, total fluorescence intensity and circularity are calculated. These quantitative parameters are presented in dot plots where each event is linked to the actual image. This enables review of the sample by means of quantitative and morphological parameters. If desired, each particular set of parameter values recorded can directly be compared to the image of the event from which they were determined.

\subsection{Results}

\subsubsection{ILLUMINATION}

The size of the flat top intensity profile created by the beam homogenizer was adjusted by slightly varying the distance between the two microlens arrays ( $\mathrm{a}_{12}$ as indicated in Figure 2.2, Panel A). Care was taken not to overfill the aperture of the lenses in the second microlens array. Overfilling results in crosstalk between the lenslets and a less sharply defined border of the flat top profile. To determine the size and the intensity profile, we used a homogeneous layer of acridine orange. The resulting illumination profile, shown in Figure 2.2, Panel B, then has a flat top with edges that are not as steep as theoretically possible, but now with a width of $180 \mu \mathrm{m}$ that can be used for imaging. During scanning, only the indicated area with a size of $180 \times 180 \mu \mathrm{m}^{2}$ is captured from the CCD camera. The intensity outside this area drops rapidly, which is important to prevent bleaching of adjacent areas when scanning the sample. If needed, the size of the illumination area can be adjusted further by choosing a different objective.

\subsubsection{RESOLUTION}

A difference in motion between the TDI CCD and the stage will result in an increase of the effective sampling aperture L and leads to blurring of the 
image in the Y direction [26]. The angular misalignment between the CCD position and the stage scan direction affects the resolution perpendicular to the scan direction. A small angular misalignment $\theta$ results in a pixel overlap, shown in Equation (2.3).

$$
L_{\text {align }}=N_{T D I} \cdot \delta p \cdot \tan (\theta)
$$

Where $\mathrm{N}_{\text {TDI }}$ is the number of TDI stages (1024) and $\delta \mathrm{p}$ is the pixel size in the scanning direction. By adjusting the CCD and the stage scan direction with accuracy of $0.03^{\circ}$ or better this blurring effect could be limited to 0.5 to 1 pixels.

During stage motion, the stage position appears to be moving with a slightly oscillating velocity $\mathrm{V}$ that has a standard deviation of $0.8 \mu \mathrm{m}$ around the commanded value. This creates a blurring pixel overlap, given by Equation (2.4).

$$
L_{v e l o c i t y}=\frac{\Delta V}{V} \cdot N_{T D I} \cdot \delta p
$$

The pixel overlap is equal to 8 pixels in case of a fixed line rate. For this reason, an encoder is used to measure the stage position continuously. The encoder triggers the camera each time when the stage position has moved by $0.2 \mu \mathrm{m}$ and thus ensures the synchronization between stage motion and CCD charge transfer. To obtain a 1:1 relation between stage movement and CCD charge transfer, the magnification was adjusted to $32.25 \times$ as accurately as possible. The residual mismatch from magnification deviation from $32.25 \times$ in pixels is shown in Equation (2.5).

$$
L_{\text {magnification }}=\frac{\Delta V}{V} \cdot N_{T D I} \cdot \delta p
$$

This mismatch appeared to result in a blurring effect of 1 pixel.

Another cause of blurring that cannot be prevented is due to the discrete movement of the charges of the TDI-CCD while the object moves continuously. This also results in a blurring of 1 pixel in the TDI scan direction.

To determine the total degradation of the resolution due to all these factors, the modular transfer function [27-30] (MTF) or spatial frequency response (SFR) was measured for the entire optical system and the TDI scanning system. From the MTF curves we can derive the actual resolution of the complete optical system. According to the Rayleigh criterion, the resolution is the inverse of the spatial frequency for which the MTF drops below $9 \%$.

A cartridge with a slanted step edge was filled with an Acridine Orange solution. The upper surface was lined with $15 \mu \mathrm{m}$ wide nickel lines with a spacing of $30 \mu \mathrm{m}$ to partly mask the fluorescence of the Acridine Orange. The slanted step edge was imaged under an angle of $\sim 5^{\circ}$ parallel and 


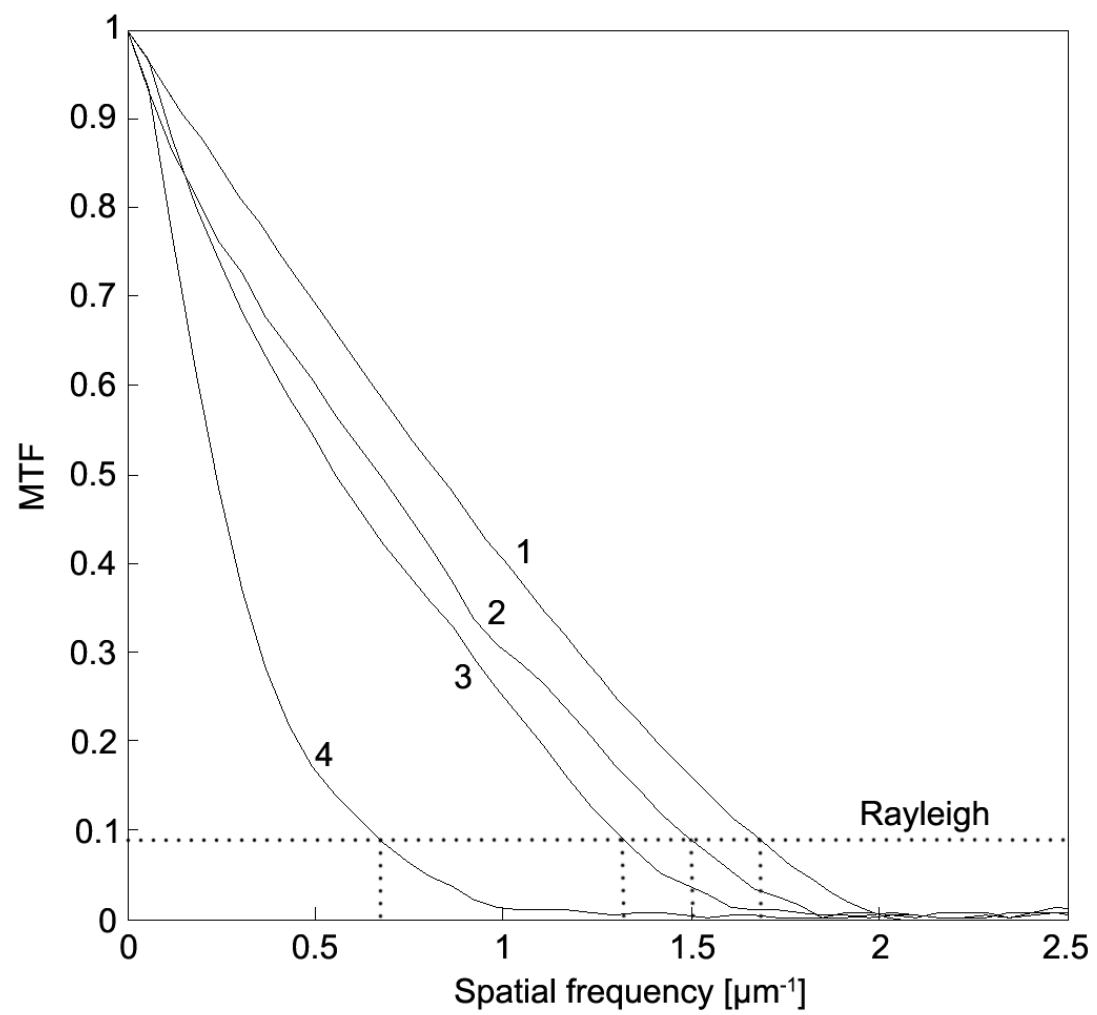

Figure 2.5: MTF of the optical system in the scan direction (Y). (1) Theoretical MTF of an $0.6 \mathrm{NA}$ objective at $580 \mathrm{~nm}$. (2) MTF with camera in frame transfer mode at $205 \mathrm{~ms}$ integration time, which is equal to the integration time for the 1024 TDI stages at $1 \mathrm{~mm} / \mathrm{sec}$ scan speed. (3) MTF with camera in TDI mode and encoder triggering at $1 \mathrm{~mm} / \mathrm{s}$. (4) MTF with camera in TDI mode and fixed line-rate triggering at $1 \mathrm{~mm} / \mathrm{s}$.

perpendicular to the scan direction to determine the MTF in both directions. To image the Acridine Orange layer, the green laser line was used for excitation in combination with a 580DF30 band pass filter. The images were analyzed with Matlab and a Slant Edge Analysis Tool, sfrmat 2.0 [31, 32]. The resulting MTF curves of the optical system in the scan (Y) direction, at a wavelength of $580 \mathrm{~nm}$, are shown in Figure 2.5.

They illustrate the difference in image degradation between the camera operating in frame transfer mode (2), TDI mode with encoder triggering (3), TDI mode with fixed line rate triggering (4), and the theoretical limit of the MTF (1). When scanning in TDI mode with encoder triggering the system has a slightly lower MTF in the scan direction as compared to acquiring images in frame transfer mode. This degradation is due to the pixel blurring and results in an approximate $10 \%$ decrease in resolution. 
Scanning at a fixed line rate of $5 \mathrm{kHz}$ at an average speed of $1 \mathrm{~mm} / \mathrm{s}$ shows severe degradation and results in about $60 \%$ decrease in resolution. This is primarily caused by a mismatch between stage speed and camera TDI row shifting. The MTF perpendicular to the scan direction is almost equal for all camera modes.

In our case, the optical resolution, when scanning using TDI mode combined with encoder triggering, at $1 \mathrm{~mm} / \mathrm{sec}$, is $0.72 \mu \mathrm{m}$ in the $\mathrm{X}$ direction and $0.76 \mu \mathrm{m}$ in the scan direction (Y) for light with a wavelength of $580 \mathrm{~nm}$. If a fixed line rate is used, the optical resolution increases to 1.5 $\mu \mathrm{m}$ in the scan direction, while the resolution in the $\mathrm{X}$ direction stays the same. When using the frame transfer mode of the camera, the resolution in the scan direction improved to $0.66 \mu \mathrm{m}$ while the resolution in the $\mathrm{X}$ direction increases slightly to $0.69 \mu \mathrm{m}$. Finally, the ideal optical system would have a resolution in both directions equal to the wavelength of the light that is used.

\subsubsection{ACQUISITION AND ANALYSIS TIME}

The total acquisition time in the CellTracks TDI is mainly determined by the scan speed. Overhead time due to stage repositioning is kept to a minimum by the use of the TDI camera. A default cartridge is scanned for 4 colors; DAPI, PE, APC and bright-field. For each color, 16 strips, $30 \mathrm{~mm}$ in length, are scanned to cover the entire analysis surface. At a default scan speed of $1 \mathrm{~mm} / \mathrm{sec}$, each color takes 8 minutes in pure scan time. Some overhead time is introduced by moving the sample to the start of a new strip and by the time it takes the stages to reach a constant velocity before scanning can begin. On average this overhead time is 1.25 minutes, leading to a total scan time including overhead for 1 color of 9.25 minutes. For 4 colors, the total scan time then amounts to 37 minutes. Without TDI camera, using a frame transfer camera, the total scan time for four colors would be 80 minutes.

Before a sample can be scanned, the borders of the cartridge and the feed forward autofocus settings have to be determined. This takes around 3 minutes, leading to a total acquisition time of around 40 minutes for 1 cartridge in 4 colors.

After the acquisition, the data is processed and analyzed as explained before. It takes 1 hour and 45 minutes to process the vast amount of data (16 GB) obtained by scanning a cartridge, with a total surface area of 81 $\mathrm{mm}^{2}$, at a sampling resolution of $0.2 \mu \mathrm{m} /$ pixel. The analysis computer is equipped with a Core2Duo processor at $2.1 \mathrm{GHz}, 4 \mathrm{~GB}$ RAM and Windows $\mathrm{XP}$. 

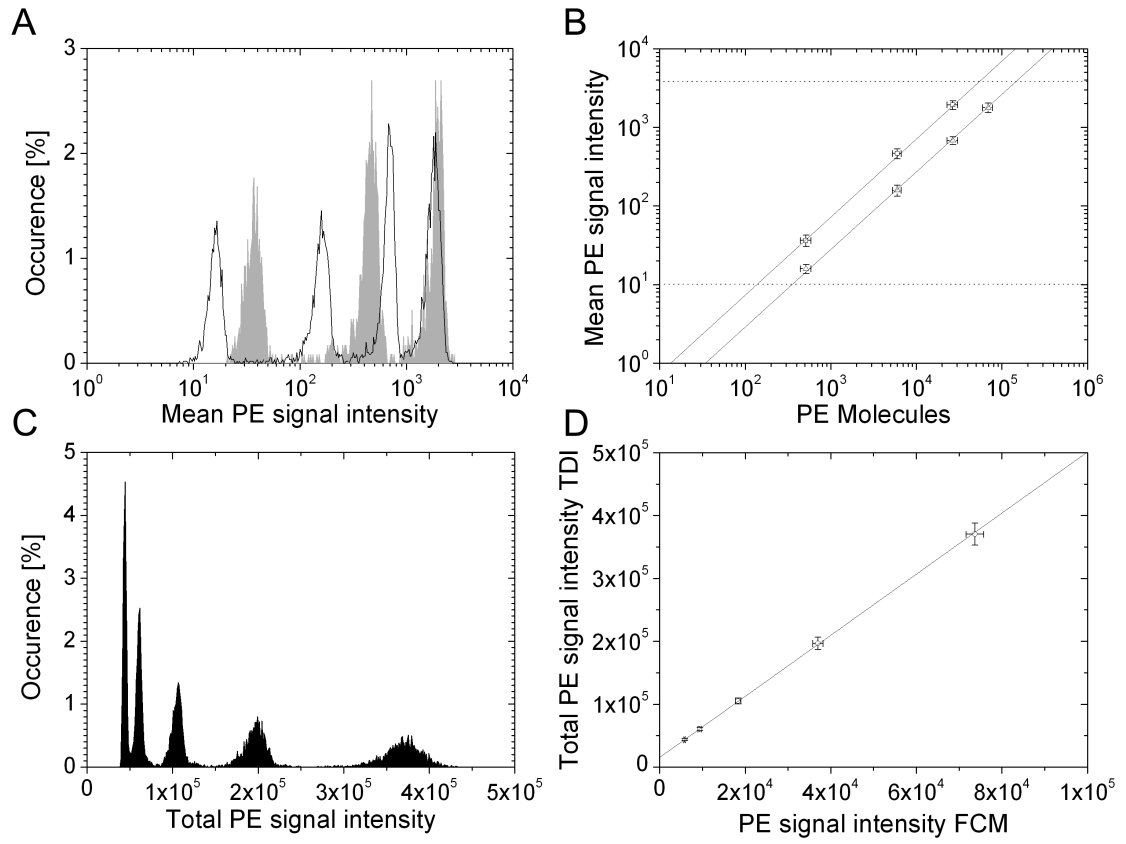

FIgURE 2.6 : (A) Histogram showing the mean PE signal intensity per bead (averaged over a circle with an area of 1020 pixels $(7.8 \mu \mathrm{m}$ diameter $)$ ), at a scan speed of $1 \mathrm{~mm} / \mathrm{sec}$. Filled grey $=$ no ND filter used, open $=$ ND 0.5 filter used to attenuate the 491 laser by a factor of $\sim 3.2$. The filled grey peak corresponding to the population with the highest number of PE molecules is not shown because a significant portion of the pixels was saturated. Coefficients of variation for the three filled grey peaks are $16.6 \%, 14.0 \%$ and $12.7 \%$ and for the open peaks $13.4 \%, 15.8 \%, 11.6 \%$ and $14.0 \%$ from left to right respectively. (B) Graph showing the mean PE signal intensities plotted against the average number of PE molecules per bead. Error bars $=1$ standard deviation, upper dotted horizontal line $=$ saturation limit (pixel value $=3895)$, lower dotted horizontal line $=3$ times the standard deviation of the background averaged over 1020 pixels (pixel value $=10.5)$. Solid lines indicate linear fits with values for $R^{2}>0.999$ in both cases. (C) Histogram of the total PE signal intensity for Rainbow RLP-30 beads. The CVs for the five peaks are, from left to right, $4.4 \%, 4.5 \%, 4.4 \%, 5.1 \%$ and $4.7 \%$ respectively. The ratio between the consecutive bead populations is $\sim 2$ as specified by the manufacturer. (D) Graph showing the total PE intensity of the beads as determined by FCM versus CellTracks TDI. The CVs as determined by FCM are, dimmest to brightest, $4.0 \%$, $3.8 \%, 3.4 \%, 3.2 \%$ and $2.8 \%$ respectively. The correlation between the two methods, as determined by a linear fit, is excellent with $\mathrm{R}^{2}=0.9998$.

\subsubsection{PERFORMANCE}

The fluorescence sensitivity of the CellTracks TDI for PE was measured using Quantibrite PE beads [33]. The beads were excited at $491 \mathrm{~nm}$ and a 580DF30 emission filter was used.

Figure 2.6, Panel A, shows histograms of the mean PE signal intensity as measured at a speed of $1 \mathrm{~mm} / \mathrm{s}$, without neutral density (ND) filter 
and with a ND 0.5 filter. The values were calculated by subtracting the background-, dark current- and bias contributions from the total signal of the object and dividing by the area of the bead (1020 pixels). All four bead populations were resolved from the background. The CV is $12 \%-16 \%$ for the four peaks and is primarily determined by a variation of the number of $\mathrm{PE}$ molecules/bead.

Measurements of the Quantibrite PE beads with a flow cytometer (FACSAria II, BD, San Jose, USA) show variations of about $13 \%$ and are thus of the same order as obtained with the CellTracks TDI. Panel B of Figure 2.6 shows the relation between the mean PE signal intensity and the known number of PE molecules. Clearly, a linear correlation exists confirming the linearity of our system. The lower limits of detection are given by the position where 3 times the standard deviation in the background coincides with the extrapolated lines. The detection limit for PE molecules on a bead with a diameter of $6.8 \mu \mathrm{m}$ at a scan speed of $1 \mathrm{~mm} / \mathrm{s}$ is $\sim 120$ without ND filter and $\sim 340$ when a ND 0.5 filter is used.

The linearity and the variation of the CellTracks TDI were measured with Rainbow linear particles scanned at a scan speed of $1 \mathrm{~mm} / \mathrm{s}$. The 491 $\mathrm{nm}$ green laser line was used for excitation after attenuating the beam by a ND 0.4 filter to prevent saturation of the brightest peak. Under these conditions the peak pixel intensity has a mean value of $\sim 1200$ which is $\sim 1 / 3$ of the full camera range. The recorded histogram is shown in Panel C.

The average CV of the peaks is $4.6 \%$ with a minimum of $4.4 \%$ for peaks 1 and 3 and a maximum for peak 4 of $5.1 \%$. The system has a linear response and a linear regression through the plot values results in $\mathrm{R}^{2}=$ 0.9998 as shown in Panel $\mathrm{D}$. The ratio between the bead populations is $\sim 2.0$ as specified by the manufacturer.

\subsubsection{CTC IMAGING}

The combination of immunofluorescence quantification and morphologic examination of CTC candidates is illustrated in Figure 2.7.

Panel A shows a dotplot of total CD45-APC intensity versus total Cytokeratin-PE intensity of events passing a threshold on the PE intensity and Panel B shows the same dotplot now with a threshold on the DAPI intensity (not shown). These quantitative values are determined by a region of interest around each event calculated by an Otsu threshold algorithm. The total intensity values are calculated by summing the intensities of all pixels inside the region of interest. The larger number of events in Panel B is mainly caused by the leukocytes staining with both DAPI and CD45-APC, but lacking Cytokeratin-PE. The larger number of events in the region staining with Cytokeratin-PE and relatively low CD45-APC staining in Panel A as compared to Panel B is caused by the omission of a DAPI threshold. Eight events are highlighted in Panels A and B and the DAPI, 

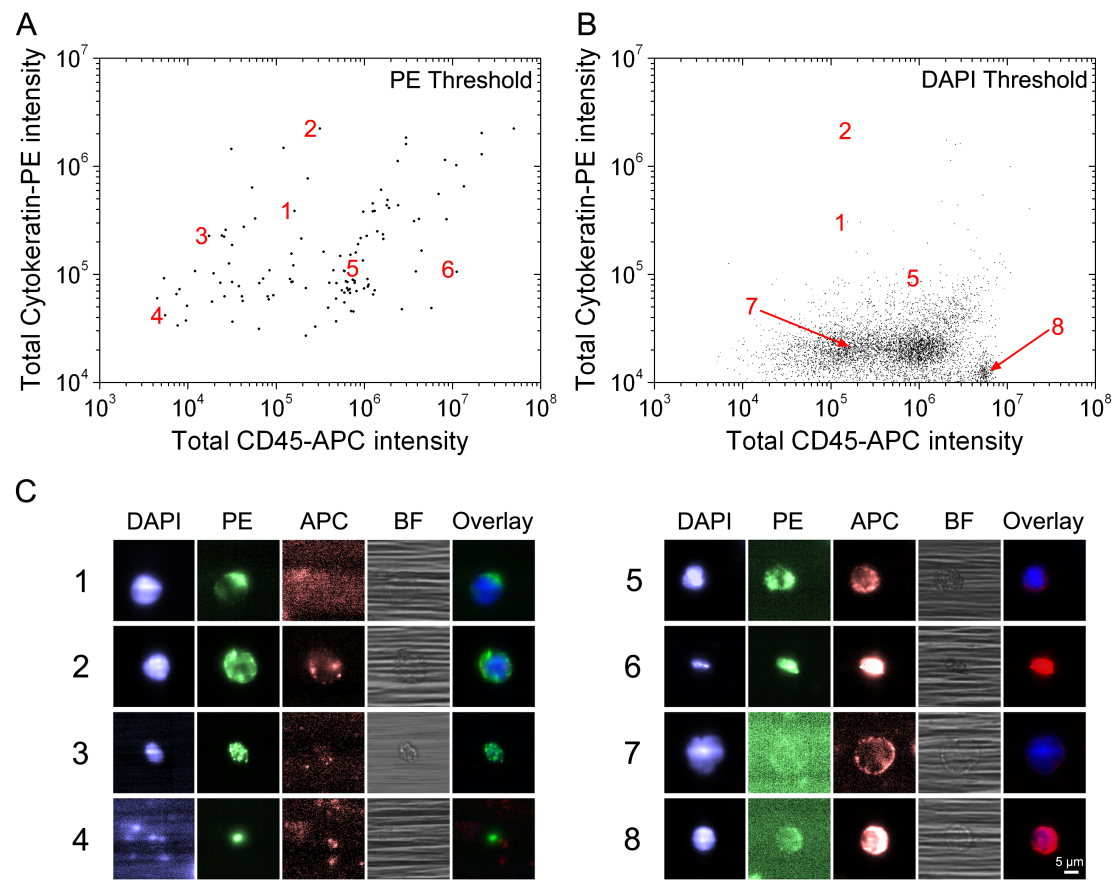

Figure 2.7 : (A) Dotplot showing total CD45-APC intensity versus total Cytokeratin$\mathrm{PE}$ intensity for all events that were detected in a single patient sample after a threshold on the PE pixel values of 50. (B) Same type of dotplot as in A, however, now the events were detected by a threshold of 50 on the DAPI pixel values. The red numbers indicate the locations of the 8 events that are shown in C. (C) Representative images from the 8 locations marked in the dotplots. Each separate image is $30 \times 30 \mu \mathrm{m}^{2}$. Scale bar represents $5 \mu \mathrm{m}$. The first column shows DAPI fluorescence in blue, second column shows Cytokeratin-PE fluorescence in green, the third column shows CD45-APC fluorescence in red, the fourth column shows the bright-field image and the last column shows an overlay of the first 3 columns. The images in the first three columns have been scaled so the color scale covers only the actual signal and not the entire camera range. This illustrates which events are close to the noise level in a certain channel, as illustrated by the PE images of events 7 and 8. The data points in $\mathbf{A}$ are three times as large as those in $\mathbf{B}$ for the sake of clarity. 


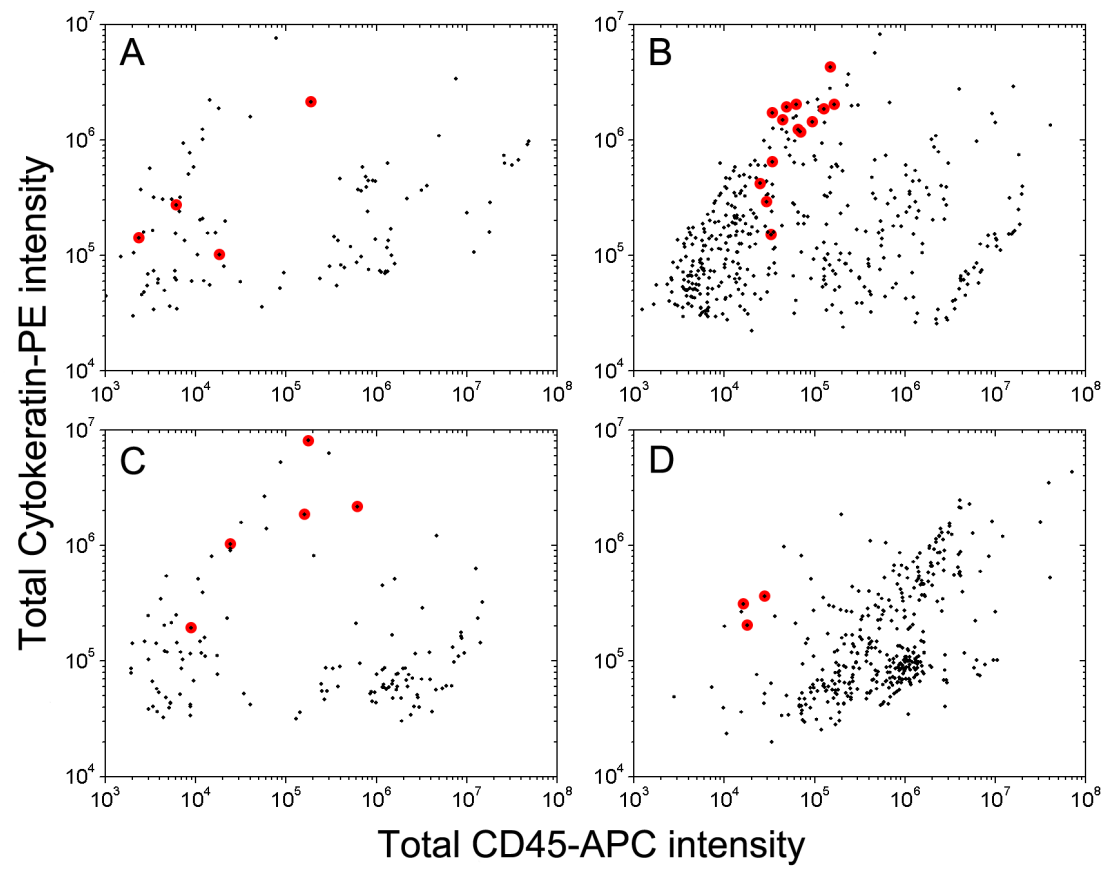

FIguRE 2.8 : (A - D) Four dotplots showing total CD45-APC signal intensity versus total Cytokeratin-PE signal intensity for events in samples from four separate metastatic cancer patients. Events were detected by a threshold on the PE pixel value of 50. All events were reviewed by an expert reviewer, following the CellSearch definition, and those that were classified as CTC are marked by red dots. The heterogeneity in these samples is clearly visible.

Cytokeratin-PE, CD45-APC, bright-field and overlay images associated with these events are shown in Panel C. Image 1 and 2 show morphological features that classify the events as CTC by the CellSearch definition [34], the cell in image 2 however shows some staining with CD45-APC, but the staining pattern is not that of a typical leukocyte. Image 3 shows an apoptotic CTC, as illustrated by the punctuated PE staining and dimmer DAPI staining than the cells in 1 and 2. Image 4 represents a tumor micro particle $[35,36]$, and image 5 resembles a leukocyte with non specific $\mathrm{PE}$ staining. Image 6 appears as debris, image 7 shows morphological features associated with a granulocyte and image 8 has morphological features associated with a lymphocyte.

Cartridges of four patients were analyzed on the CellTracks TDI and the results are shown in Figure 2.8. Events that were classified as CTC by an expert reviewer, according to the CellSearch definition, are indicated as red dots and are surrounded by events that were not classified as CTC. The CellSearch definition contains morphological and size criteria, e.g. 
round to oval morphology and minimum size of the cell. The four dotplots illustrate that CTC do not appear in a confined region in a CD45-APC versus Cytokeratin-PE dotplot. This illustrates the importance of having high resolution fluorescence- and bright-field images of events in a patient sample for visual confirmation and classification by an expert reviewer to obtain a reliable CTC enumeration.

\subsection{Discussion}

A fully automated image cytometer was developed for routine analysis of samples in which only very few positive events are expected amidst debris and leukocytes. In this study, the system was optimized for the detection of immunomagnetically enriched CTC from blood of cancer patients. For this application the definition of a CTC is of importance making it imperative to avoid false positive events or miss true positive events. This means that for identification we have to use both morphological- and immunofluorescence parameters, determined at relatively high resolution and sensitivity. The time needed for analysis should be within limits that are reasonable for routine application in a clinical chemistry setting. Therefore, in designing the cytometer, a compromise had to be made between resolution, analysis time, sensitivity, and number of measured parameters. Also the overhead time in scanning and analyzing had to be minimized. The image cytometer has a proven scan resolution of $0.76 \mu \mathrm{m}$, for light with a wavelength of 580 $\mathrm{nm}$, and images three different fluorescent- and one bright-field channel for a cartridge. It scans a surface of $2.7 \times 30 \mathrm{~mm}^{2}$ in 9.25 minutes for each parameter amounting to 37 minutes for DAPI, PE, APC and bright-field combined. The sensitivity of the optical system is $120 \mathrm{PE}$ molecules at a default scan speed of $1 \mathrm{~mm} / \mathrm{sec}$.

These results are obtained by using three laser lines, a $40 \times / 0.6 \mathrm{NA}$ objective and a beam homogenizer to create a high intensity square homogeneous illumination profile for fluorescence excitation of a wide range of fluorophores. Different laser lines can be added to the system, provided that a suitable multiple band dichroic filter can be produced. A CCD camera operating in TDI mode is used to lower the overhead time in scanning and to increase the sensitivity. TDI scanning is performed in a continuous motion, in synchronization with the scanning of the stages. Microscope based systems that use lasers, such as confocal microscopes and laser scanning cytometers, use a scanning mirror for illumination. The advantage of using a beam homogenizer, as in our system, is the simultaneous homogeneous illumination of the entire field of view. The combination of laser illumination and beam homogenization allows for control over the laser power in a well defined area, thereby avoiding bleaching of part of the sample that is not currently being imaged. Using a laser with e.g. double the output power would also double the sample illumination, something which is not 
possible in mercury arc lamp systems. This would reduce the integration time needed for the dyes that are used in the CellTracks TDI by a factor of 2 as these are well away from being limited by bleaching [18]. Although mercury arc lamps are frequently used as a light source in fluorescence microscopes, the advantage of having multiple excitation wavelengths is overshadowed by the limited lifetime of the bulb, the need for realignment and the inhomogeneous illumination. The latter could be overcome by the use of a beam homogenizer.

A high power LED provides the illumination for bright-field imaging in the image cytometer. However, image quality in the bright-field images is not optimal as can be seen in Figure 2.7, Panel C. A barcode like pattern is visible in these images. This is due to the unbound magnetic particles that are present in the sample, which are also moved to the analysis surface by the magnetic field. There they form these characteristic patterns that obstruct clear bright-field imaging. A quantitative and qualitative analysis of the effect of unbound magnetic particles and a method to remove them from a sample are discussed in Chapters 6 and 7 .

The image cytometer allows us to detect immunomagnetically enriched and fluorescently labeled CTC quickly and accurately from blood samples of patients. Quantitative measurement of fluorescence in combination with morphological examination permits for a better discrimination within the CTC candidates. Thus, it creates new opportunities for a more accurate determination of the clinical meaning of CTC in blood of cancer patients. For rare event detection, flow cytometry based systems do not analyze the complete volume of the sample. This shortcoming is addressed in the image cytometer by the use of an analysis cartridge in which all the magnetically labeled cells in the sample are moved to the analysis surface. Moreover, the analysis cartridge provides the opportunity to revisit cells after initial analysis. The system can automatically relocate events of interest and perform further detailed analysis, even under different sample conditions. It can create Z-stacks of images of selected events to perform 3D analysis and record time-lapse series. This can be used to extract more information from the analyzed cells such as the position of intracellular structures or FISH probes [37] which for example cannot be achieved by flow cytometry based systems.

Future improvements of the cytometer will include reduction of image acquisition time of multiple fluorophores by parallel imaging. This requires the addition of one or more TDI cameras and a beam splitting element to the setup. We are currently in the process of adding a second TDI camera to the system to reduce imaging times by a factor of 2 . Alternatively, a TDI camera may be used in which different areas on the CCD can be read out separately such as used in the ImageStream system [38]. A different option is to use a TDI camera operating at a higher line rate. The maximum line rate of the current TDI camera is $8.8 \mathrm{kHz}$, resulting in a theoretical maximum scan speed of $1.76 \mathrm{~mm} / \mathrm{sec}$. A newer TDI camera, now available 
from the same manufacturer, features a maximum line rate of $31 \mathrm{kHz}$, raising the theoretical maximum scan speed to $6.2 \mathrm{~mm} / \mathrm{sec}$. This is almost a factor of 4 higher than the current maximum scan speed. The latter option would also allow the use of higher laser power for illumination, which is not possible now because of the saturation of pixels at higher intensities. If desired, a fourth fluorescence parameter can be added. This requires the use of a quad-band dichroic filter. A drawback is that such filters often have lower efficiency for the separate dyes as compared to a triple band dichroic. Apart from the mentioned hardware changes, the image analysis can be made faster by using a separate dedicated analysis computer with multiple processors at high clock speed.

We estimate that the overall reduction in time needed for analyzing a cartridge of $2.7 \times 30 \mathrm{~mm}^{2}$ using three fluorescence- and one bright-field channel could be a factor of 8 , bringing the time needed to analyze one sample well below 5 minutes.

Even though we designed and built the image cytometer in the first place to improve the method to determine CTC in blood samples, it is obvious that many applications of the instrument exist. In fact, the cytometer can be adapted easily for the analysis of other cells where in a routine setting high resolution imaging and quantitative multispectral fluorescence characterization of cellular samples need to be combined.

\section{ACKNOWLEDGEMENTS}

This work was supported by Veridex, LLC, Raritan, NJ, USA.

\subsection{REFERENCES}

[1] E. Luther and L. Kamentsky, "Resolution of mitotic cells using laser scanning cytometry," Cytometry, vol. 25, pp. 272-278, 1996.

[2] L. Dietz, R. Dubrow, B. Manian, and N. Sizto, "Volumetric capillary cytometry: A new method for absolute cell enumeration," Cytometry, vol. 23, pp. 177-186, 1996.

[3] A. Tibbe, B. de Grooth, J. Greve, P. Liberti, G. Dolan, and L. Terstappen, "Optical tracking and detection of immunomagnetically selected and aligned cells," Nature Biotechnology, vol. 17, pp. 1210-1213, 1999.

[4] T. George, D. Basiji, B. Hall, D. Lynch, W. Ortyn, D. Perry, M. Seo, C. Zimmerman, and P. Morrissey, "Distinguishing modes of cell death using the imagestream multispectral imaging flow cytometer," Cytometry Part A, vol. 59(2), pp. 237-245, 2004.

[5] E. Racila, D. Euhus, A. Weiss, C. Rao, J. McConnell, L. Terstappen, and J. Uhr, "Detection and characterization of carcinoma cells in the blood," PNAS, vol. 95, pp. 4589-4594, 1998.

[6] L. Terstappen, C. Rao, S. Gross, and A. Weiss, "Peripheral blood tumor cell load reflects the clinical activity of the disease in patients with carcinoma of the breast," Int J Onc, vol. 17(3), pp. 573-578, 2000. 
[7] L. Terstappen, Hematopoietic Stem Cell Transplantation, ch. Detection of infrequent cells in blood and bone marrow by flowcytometry, pp. 137-152. Marcel Dekker Inc, 2000 .

[8] M. Cristofanilli, T. Budd, M. Ellis, A. Stopeck, J. Matera, M. Miller, J. Reuben, G. Doyle, W. Allard, L. Terstappen, and D. Hayes, "Circulating tumor cells, disease progression, and survival in metastatic breast cancer," N Engl J Med, vol. 351(8), pp. 781-791, 2004.

[9] S. Cohen, C. Punt, N. Iannotti, B. Saidman, K. Sabbath, N. Gabrail, J. Picus, M. Morse, E. Mitchell, C. Desch, M. Miller, G. Doyle, H. Tissing, L. Terstappen, and N. Meropol, "The relationship of circulating tumor cells to tumor response, progression-free survival, and overall survival in patients with metastatic colorectal cancer," Journal of Clinical Oncology, vol. 26(19), pp. 3213-3221, 2008.

[10] J. De Bono, H. Scher, R. Montgomery, C. Parker, M. Miller, H. Tissing, G. Doyle, L. Terstappen, K. Pienta, and D. Raghavan, "Circulating tumor cells predict survival benefit from treatment in metastatic castration resistant prostate cancer," Clin Can Res, vol. 14(19), pp. 6302-6309, 2008.

[11] B. Cairns and E. Wolf, "Changes in the spectrum of light scattered by a moving diffuser plate," J Opt Soc Am, vol. 8(12), pp. 1922-1928, 1991.

[12] T. Hirai, K. Fuse, K. Kurisu, and K. Ebata, "Development of diffractive beam homogenizer," SEI Technical review, vol. 60, pp. 17-23, 2005.

[13] C. Kopp, L. Ravel, and P. Meyrueis, "Efficient beamshaper homogenizer design combining diffractive optical elements, microlens array and random phase plate," $J$ OP A :Pure Appl Opt, vol. 1, pp. 398-403, 1999.

[14] A. Riser and W. Cassarly, "Analysis of single lens arrays using convolution," Optical Engineering, vol. 40(5), pp. 805-813, 2001.

[15] P. Schreiber, S. Kudaev, and P. Dannberg, "Homogeneous led-illumination using microlens arrays," www.microoptics.org, vol. -, pp. -, -.

[16] R. Volkel, M. Eisner, and W. KJ, "Miniaturized imaging systems," Microelectronic Engineering, vol. 67, pp. 461-472, 2003.

[17] S. MicroOptics, "Smo techinfo sheet 10 -beam homogenizing," http://www.sussmicrooptics.com/downloads/SMO echInfo $_{S}$ heet ${ }_{1} 0 . p d f$, vol. - , pp. - - - - .

[18] F. Schreuder, Laser image cytometer for analysis of circulating tumor cells. PhD thesis, PhD Thesis University of Twente, ISBN 9789036526104, 2008.

[19] W. Rasband, "Imagej," http://rsbweb.nih.gov/ij/index.html, vol. -, pp. -, 1997 2010.

[20] M. Abramoff, P. Magelhaes, and S. Ram, "Image processing with ImageJ," Biophotonics International, vol. 11(7), pp. 36-42, 2004.

[21] W. Bocker, H. Gantenberg, W. Muller, and C. Streffer, "Automated cell cycle analysis with fluorescence microscopy and image analysis," Phys Med Biol, vol. 41(3), pp. 523-537, 1996.

[22] M. Model and J. Burkhardt, "A standard for calibration and shading correction of a fluorescence microscope," Cytometry, vol. 44(4), pp. 309-316, 2001.

[23] V. Varga, J. Bocsi, J. Sipos, G. Csendes, Z. Tulassay, and B. Molnar, "Scanning fluorescent microscopy is an alternative for quantitative fluorescent cell analysis," Cytometry Part A, vol. 60(1), pp. 53-62, 2004.

[24] S. Sternberg, "Biomedical image processing," Computer, vol. 1, pp. 22-34, 1983. 
[25] P. Liao, T. Chen, and P. Chung, "A fast algorithm for multilevel thresholding," Journal of Information Science and Engineering, vol. 17(5), pp. 713-727, 2001.

[26] H. Wong, Y. Yao, and E. Sclilig, "TDI charge coupled devices: design and applications," IBM J Res Dev, vol. 36, pp. 83-106, 1992.

[27] P. Burns, "Slanted-edge mtf for digital camera and scanner analysis," Proc PICS Conf ISET T, vol. -, pp. 135-138, 2000.

[28] E. Stevens, "A unified model of carrier diffusion and sampling aperture effects on mtf in solid-state image sensors," IEEE transactions on electron devices, vol. 39(11), pp. 2621-2623, 1992.

[29] T. Fischer and J. Holm, "Electronic still picture camera spatial frequency response measurement," Proc PICS Conf ISEST, vol. 2, pp. 626-630, 1994.

[30] "Iso 12233 international standard, photography - electronic still picture cameras resolution measurements."

[31] P. Burns and D. Williams, "Using slanted edge analysis for color registration measurements," Proc PICS Conf ISEST, vol. -, pp. 51-53, 1999.

[32] P. Burns and D. Williams, "Refined slanted-edge measurements for practical camera and scanner testing," Proc PICS Conf ISEST, vol. -, pp. 191-195, 2002.

[33] E. Chase and R. Hoffman, "Resolution of dimly fluorescent particles: A practical measure of fluorescence sensitivity," Cytometry, vol. 33(2), pp. 267-279, 1998.

[34] W. Allard, J. Matera, M. Miller, M. Repollet, M. Connelly, C. Rao, A. Tibbe, J. Uhr, and L. Terstappen, "Tumor cells circulate in the peripheral blood of all major carcinomas but not in healthy subjects or patients with nonmalignant diseases," Clinical Cancer Research, vol. 10(20), pp. 6897-6904, 2004.

[35] C. Larson, J. Moreno, K. Pienta, S. Gross, M. Repollet, S. OHara, T. Russell, and L. Terstappen, "Apoptosis of circulating tumor cells in prostate cancer patients," Cytometry part A, vol. 62(1), pp. 46-53, 2004.

[36] F. Coumans, C. Doggen, G. Attard, J. de Bono, and L. Terstappen, "All circulating $\mathrm{EpCAM}+\mathrm{CD} 45-\mathrm{CK}+$ but not $\mathrm{EpCAM}+\mathrm{CD} 45+\mathrm{CK}+$ objects predict overall survival in castration-resistant prostate cancer," Annals of Oncology, vol. 21(9), pp. 18511857,2010

[37] J. Swennenhuis, A. Tibbe, R. Levink, R. Sipkema, and L. Terstappen, "Characterization of circulating tumor cells by fluorescence in-situ hybridization," Cytometry Part A, vol. 75(6), pp. 520-527, 2009.

[38] W. Ortyn, B. Hall, T. George, K. Frost, D. Basiji, D. Perry, C. Zimmerman, D. Coder, J. Philip, and P. Morrissey, "Sensitivity measurement and compensation in spectral imaging," Cytometry Part A, vol. 69(8), pp. 852-862, 2006. 


\section{Automated IDENTIFICATION OF Circulating Tumor Cells By IMAGE CYTOMETRY}

Presence of Circulating Tumor Cells (CTC), as detected by the CellSearch ${ }^{\circledR}$ System, in patients with metastatic carcinomas is associated with poor survival prospects. CellTracks TDI, a dedicated image cytometer, was developed to improve the enumeration of these rare CTC.

The CellSearch System was used to enumerate CTC in $7.5 \mathrm{ml}$ blood of 68 cancer patients and 9 healthy controls. Cartridges containing the fluorescently labeled CTC from this system were reanalyzed and automated classification of events was performed by the Random Forest method using Matlab.

An automated classifier was developed to classify events into CTC, apoptotic CTC, CTC debris, leukocytes and debris not related to CTC. A high agreement in classification was obtained between the automated classifier and 5 expert reviewers. Improved detection efficiency for CD45-APC avoids the classification of leukocytes non-specifically binding to cytokeratin as CTC. The correlation between the number of CTC detected in CellTracks TDI and CellTracks Analyzer II is good with a slope of 1.88 and a correlation coefficient of 0.87 .

Automated classification of events by CellTracks TDI eliminates the operator error in classification of events as CTC and permits quantitative assessment of parameters. The clinical relevance of various CTC definitions can now be investigated.

This chapter has been published in Cytometry A, 2012 doi: 10.1002/cyto.a.22002 


\subsection{INTRODUCTION}

CTC may be present in the peripheral blood of cancer patients and their presence is associated with a reduced probability of survival [1-6]. Reports on the number of tumor cells present in peripheral blood vary greatly between studies [7-14]. This variation can be mainly attributed to the differences in sample preparation and analysis techniques. To minimize variability in rare cell analysis, standardized and automated sample preparation is essential. In the analysis of the samples, the difficulty is to set criteria by which an event can be classified as a tumor cell and which can be applied across samples, instruments and operators. In this study, CTC were immunomagnetically enriched for expression of Epithelial Cell Adhesion Molecule (EpCAM) from $7.5 \mathrm{ml}$ blood samples and fluorescently labeled with CD45-APC, Cytokeratin-PE (CK-PE) and DAPI with an automated sample preparation system $[14,15]$. Analysis of the samples is performed by the CellTracks Analyzer II, a semi-automated fluorescence microscope that presents images of events staining with CK-PE and DAPI to the reviewer for CTC classification [14, 15]. The heterogeneity in the morphology of the CTC candidates, mainly caused by a variable degree of apoptosis, makes their classification difficult $[13,16,17]$. In this study we introduce an image cytometer that uses lasers and a Time Delay Integration (TDI) camera to achieve automated classification of CTC using the Random Forest method [18, 19].

\subsection{Materials And Methods}

\subsubsection{PATIENTS AND CONTROLS}

CTC were enumerated in thirty-one blood samples from patients with primary breast or colorectal cancer, thirty-seven samples from patients with metastatic carcinomas and nine samples from healthy donors. All patients provided informed consent and the use of samples was approved by the ethics board of Medisch Spectrum Twente, Enschede, the Netherlands.

\subsubsection{SAMPLE PREPARATION}

Blood was drawn in $10 \mathrm{ml}$ CellSave Preservative evacuated blood collection tubes (Veridex LLC, Raritan, NJ). Samples were kept at room temperature and processed within 72 hours. $7.5 \mathrm{ml}$ aliquots of blood were centrifuged and placed in a CellTracks ${ }^{\circledR}$ AutoPrep ${ }^{\circledR}$ System. This system uses EpCAM antibody labeled ferrofluids to immunomagnetically enrich cells of epithelial origin. The magnetically enriched cells are fluorescently labeled with the nucleic acid dye DAPI, Phycoerythrin labeled monoclonal antibodies directed against Cytokeratin 8, 18 and 19 (CK-PE) and Allophycocyanin labeled monoclonal antibodies against CD45 (CD45-APC). After incubation, the 
sample is transferred into a CellTracks analysis cartridge which is present inside the MagNest ${ }^{\circledR}$ cell presentation device. This device presents the magnetically labeled cells at the upper surface of the sample cartridge [20].

\subsubsection{CELLTRACKS ANALYZER II}

The CellTracks Analyzer II is a four color, semi-automated fluorescence microscope (Veridex LLC, Raritan, NJ). The analyzer uses a $10 \times / 0.45 \mathrm{NA}$ microscope objective in combination with a CCD camera to acquire fluorescence images covering the entire surface of the sample chamber. After image analysis, a gallery of objects stained positive for both CK-PE and DAPI is shown to the operator. A trained operator makes the final decision whether the presented image classifies as a CTC. The CTC criteria require the event to have a diameter of at least $4 \mu \mathrm{m}$, round to oval morphology, positive staining for CK-PE, a clearly defined nucleus and negative staining for CD45-APC [14]. CD45-APC is added to the assay to avoid classification of leukocytes non-specifically staining with CK-PE as CTC.

\subsubsection{CELLTRACKS TDI ANALYZER}

After the initial scan with the CellTracks Analyzer II, the analysis cartridge is kept inside the MagNest and the sample is rescanned the same day using the CellTracks TDI system $[18,19]$. To increase the resolution and sampling density, the CellTracks TDI uses a $40 \times / 0.6 \mathrm{NA}$ objective, instead of the $10 \times / 0.45$ NA objective that is used in the CellTracks Analyzer II. TDI image acquisition is used to obtain fluorescence images while the cartridge is scanned through the imaging plane, which minimizes image acquisition time. The use of TDI for image acquisition in cytometry is also incorporated in the ImageStream system [21]. Epi-fluorescence illumination is performed using three different laser lines with wavelengths of 375, 532 and $639 \mathrm{~nm}$. Beam homogenizing optics (Suss-MicroOptics, Neuchatel, Switzerland) are used to create a square $180 \times 180 \mu \mathrm{m}^{2}$ homogeneous illumination profile. Bright-field images were obtained by backlight illumination using a blue LED, which was placed below the analysis cartridge. For each sample, 12 bit raw images of the whole cartridge surface are acquired for DAPI, CK-PE, CD45-APC and bright-field.

After image acquisition, the initial 16.1 GB image data per sample is processed by an ImageJ script [22, 23]. This script performs a correction for inhomogeneous illumination and subtracts the background from all images. The signal level of the CK-PE images is used as a threshold. Image locations with a CK-PE pixel intensity of equal to or greater than 50 above the noise level and that are larger than $9 \mu^{2}$ are marked as possible locations for a CTC. Next, at the marked image locations an area of $30 \times 30 \mu \mathrm{m}^{2}$ is saved for DAPI, CK-PE, CD45-APC and bright-field. This smaller data set, with 
a size of $\sim 172 \mathrm{Mb}$ for 1000 events, is processed further and the remaining image data is discarded.

The script then determines a region of interest (ROI) for each CK-PE event by means of an Otsu threshold algorithm [24]. This ROI is used as a measure for the object size and serves as a mask to determine quantitative parameters for an event. The corresponding ROI for the nucleus stained with DAPI is obtained with the same Otsu threshold algorithm with the restriction that the center of this $\mathrm{ROI}$ is restricted to the area of the CK-PE ROI. The center of the nucleus has to be located within a radius of $10 \mu \mathrm{m}$ of the center of the CK-PE ROI and the size is limited to 1.5 times the size of the CK-PE ROI. If no object is found within these restrictions, the same ROI for DAPI is used as for the CK-PE image. The same restrictions hold for the CD45-APC images. These restrictions assure the presence of a ROI for each object and prevent the selection of ROI's for different colors that do not belong to the same event. The size restriction with respect to the CK-PE ROI is necessary to prevent selection of the whole $30 \times 30 \mu \mathrm{m}^{2}$ image if no objects are found. For each event, using the ROI's for DAPI, CK-PE and CD45-APC, several parameters are calculated in the following categories: quantitative, correlation, texture and morphological. These parameters are then used in a classification algorithm to obtain automated event classification.

\subsubsection{AUTOMATED EVENT CLASSIFICATION}

The basis for automated classification is the availability of a manually classified number of events that are labeled as training- and test set. We used an expert reviewer to classify events in the training and test set into 5 different classes. These are defined as: 1. CTC; DAPI positive, CK-PE positive, CD45-APC negative events with a round to oval nucleus, at least a large part of the cell lined with CK-PE and a nucleus inside the cytoskeleton; 2. Apoptotic CTC; events with an intensity of the DAPI fluorescence that is significantly less as compared to CTC and CK-PE staining with a dotted pattern; 3. CTC debris; consisting of small events, less than $4 \mu \mathrm{m}$ in diameter, CK-PE positive, but DAPI negative and CD45-APC negative; 4. Leukocytes; DAPI positive, CK-PE dim, CD45-APC positive events with round to oval morphology and a nucleus inside the CD45-APC pattern; 5 . Debris; representing anything other than cells of interest, usually aggregates of staining reagents or debris in the sample or destroyed cells.

The automated classifier was developed in Matlab 2009a (Mathworks, Natick, MA). For classification of events we made use of the Random Forest (RF) method [25-29]. This classification method has been used previously to classify hyper-spectral [30], bright-field [31], SPECT [32] and fluorescence images [33, 34]. The RF method is an ensemble classifier that uses multiple classification trees for decision making. Each tree uses a random subset of the parameters and events present in the entire training set. At each 
node in the decision tree, a best split is made based on a single parameter. The tree is fully grown until all the parameters are used to form nodes in the tree. Events that were not used to grow the tree are run down the tree to determine the "out-of-bag" (OOB) error rate. This serves as a method for internal cross validation. Since RF classification is an ensemble classifier, multiple trees are grown and each tree votes for a specific class for each event. The total RF classification method uses many trees to achieve balanced voting.

In this classifier we used 5000 trees. This number generated a vote distribution that did not significantly change with the addition of more trees to the forest. This results in a majority vote for each event that is classified by the RF method, which determines its final class. The RF method is versatile as it accepts both categorical and continuous parameters. Also, internal cross-validation is quite accurate in predicting the accuracy of the final classifier, which is defined as the number of correctly determined events, in a single class or in all classes, by the RF classifier divided by the total number of events in the same class. Furthermore, variables like the importance of separate parameters and a measure to determine outliers in the data can give more insight in the data set.

To determine the importance of a parameter, its values are randomized and the effect on classification is determined. Parameters that have a large effect on the classification accuracy are said to have high importance. On the contrary, parameters that have a low importance might even be removed from the parameter set without having a significant influence on the classification accuracy. The outlier measure is a value that determines how many standard deviations an event is from the center of that population. Events that are e.g. 6 or more standard deviations from the median of the population can be further examined to check the correctness of the manual classification. The final RF classifier consists of many trees that all vote for a specific class for each event. A parameter, termed margin, can be constructed that quantifies the "likelihood" of each class for a specific event. It is defined as the difference between the fractions of votes for the majority class minus the fraction of votes for the second largest class. Large values denote that the classifier is relatively sure about the classification, although it does not take into account whether the classification is correct, i.e. the same as the manual classification by the expert reviewer.

\subsection{Results}

\subsubsection{CELLTRACKS TDI DATA ANALYSIS}

CellTracks TDI identifies events at the surface of the analysis cartridge that have passed a threshold on CK-PE and measures several morphological parameters and quantitative fluorescence parameters from these events. The data set for the development of the automated event classification consisted 
of 11872 events classified by an expert reviewer from 68 patient samples and 153 parameters were determined for each event. Data from 7698 events was used as a training set and the remainder was used as the test set. Events in patient samples were randomly assigned to either the test or training set. Two variables related to the RF classification process were optimized during training of the classifier. Mtry, which is the number of parameters considered for each tree and sampsize, which is the number of events (same for all 5 classes) that were randomly selected from all the events in the training set. For mtry the value was varied over the interval of 1-24 and the optimal value was 12 as it generated the lowest average internal error rate. This is very close to the default value of mtry that is recommended, which is the square root of the number of parameters.

Default RF classifiers randomly select about one third of all the events during construction of a new tree. For imbalanced data sets, as is the case here, this is not optimal [35]. This is due to the fact that the RF classifier tries to minimize the total error rate during training. Because the most frequent classes are leukocytes, debris and CTC debris, this would favor the correct classification of these classes. This implies that correct classification of less frequent classes is less important. Because the least frequent classes (CTC and apoptotic CTC) are the most important to get correct, a different sampling method has to be used during creation of a new tree, other than random sampling. The best classification is obtained when no class is favored during creation of a tree, resulting in the need for equal numbers of events from all 5 classes. This is determined by the variable sampsize. To determine the optimal value, it was varied over the range of 10-230 with intervals of 10 . A smaller interval did not significantly improve the determination of the optimal value, but it did significantly increase computation time. The optimal value for sampsize was found to be 110 .

The importance of individual parameters for correct classification of a specific type of event is illustrated for CTC and leukocytes in Table 3.1. The higher the number, the more important the feature is for classification. The ratio of CK-PE total intensity divided by CD45-APC total intensity is, with a value of 12.6 , by far the most important in classification of CTC. For leukocytes, the slope of the correlation between DAPI and CK-PE intensity is, with a value of 10.0, the most important. Analyzing parameters by their relative importance allows for optimization of parameters by removing unimportant parameters and possibly adding parameters in more important classes.

The final RF classifier was trained using the optimized parameters. OOB error rates were: CTC: $17.4 \%$, apoptotic CTC: $33.8 \%$, CTC debris: $9.4 \%$, Leukocytes: $5.5 \%$, Debris: $10.9 \%$ and total: $9.9 \%$. To determine actual performance of the RF classifier, it was used on the test set which consists of 4174 events. The resulting classes were compared to the manual classes as determined by the expert reviewer. The error rates are: CTC: $10.2 \%$, apoptotic CTC: $34.1 \%$, CTC debris: $9.5 \%$, Leukocytes: $4.0 \%$, Debris: $10.8 \%$ 


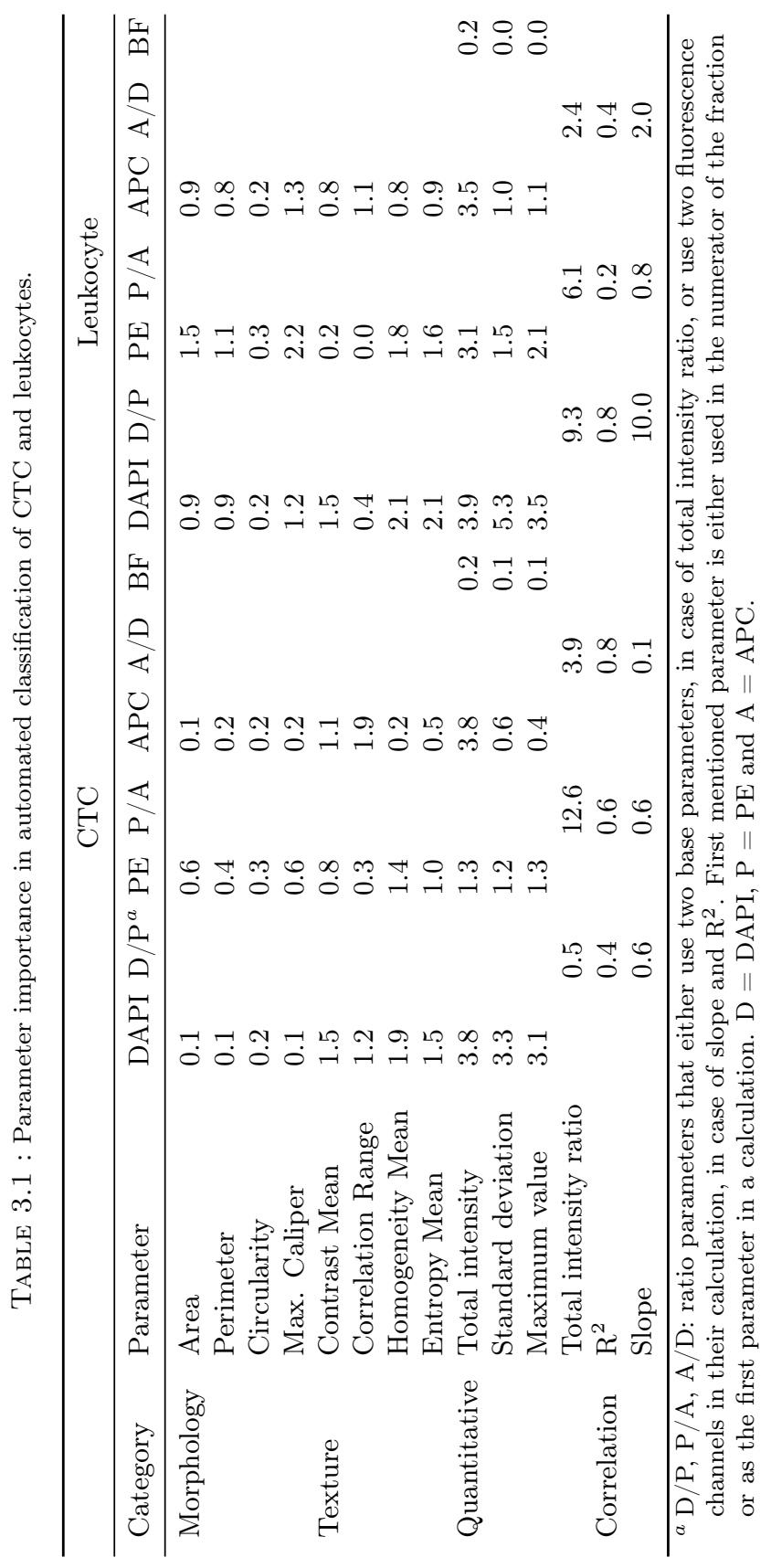


A

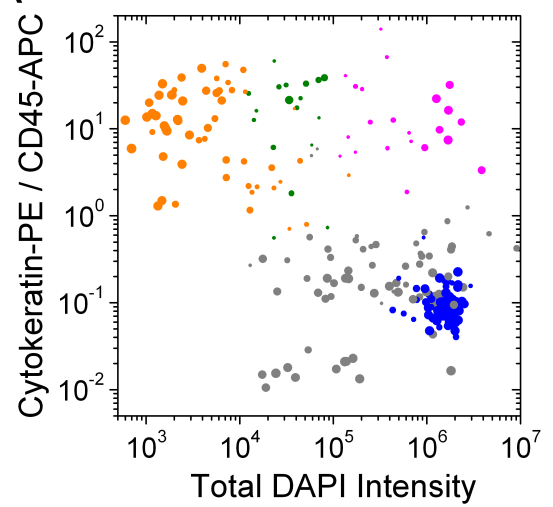

B

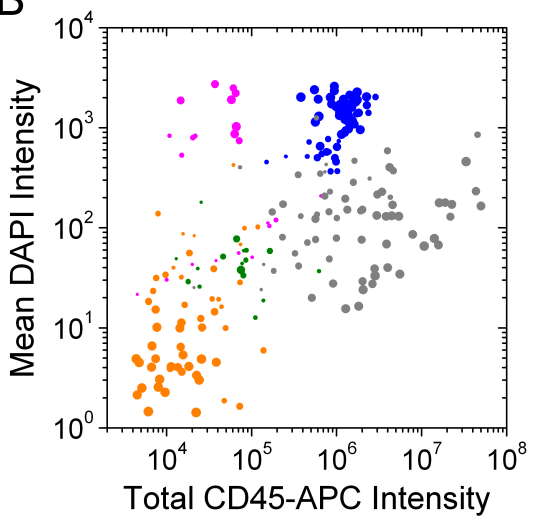

FIguRE 3.1 : Scatter plots of CellTracks TDI CTC analysis of $7.5 \mathrm{ml}$ of blood from a metastatic carcinoma patient showing the 4 parameters with the highest importance during training of the RF classifier. The size of each dot varies and depicts the margin of the automated classification of the event. A larger dot indicates a higher confidence. In total, 246 events are shown in the figure; 22 CTC colored magenta, 18 apoptotic CTC colored green, 62 CTC debris colored orange, 75 leukocytes colored blue and 69 events debris colored dark grey.

and total: $9.6 \%$. To compare the classification by the expert reviewer and the automated classifier, a confusion matrix was constructed to illustrate the number and percentage of events that were classified into each class by the expert reviewer and the automated classifier, which is shown in Table 3.2. Results from all classified events are given in the table as well as a separation into the training and test set. Values between brackets indicate the percentage of events in a specific field based on the total number of events as classified by the expert reviewer. Percentages on the diagonals of the three sub-tables indicate the recall rate from a specific class. Recall rate is defined as the number of events, for a specific class, correctly classified by the RF classifier divided by the total number of events determined to be in that class by the expert reviewer. Recall rate is a measure of sensitivity. Another value that can be calculated from Table 3.2 is precision. It is defined as the number of events, for a specific class, correctly classified by the RF classifier divided by the total number of events determined to be in that class by the RF classifier, giving a measure of specificity. Precision values calculated from the total data set are (range 0-1): 0.50 for CTC, 0.23 for apoptotic CTC, 0.87 for CTC debris, 0.86 for leukocytes and 0.98 for debris. This shows that few events are falsely classified as debris, however, some events that are debris are incorrectly classified as CTC related events.

An example of a measurement of a sample from a metastatic carcinoma patient is shown in Figure 3.1. Panel A shows the total DAPI intensity versus the ratio of the total CK-PE intensity divided by the total CD45-APC 


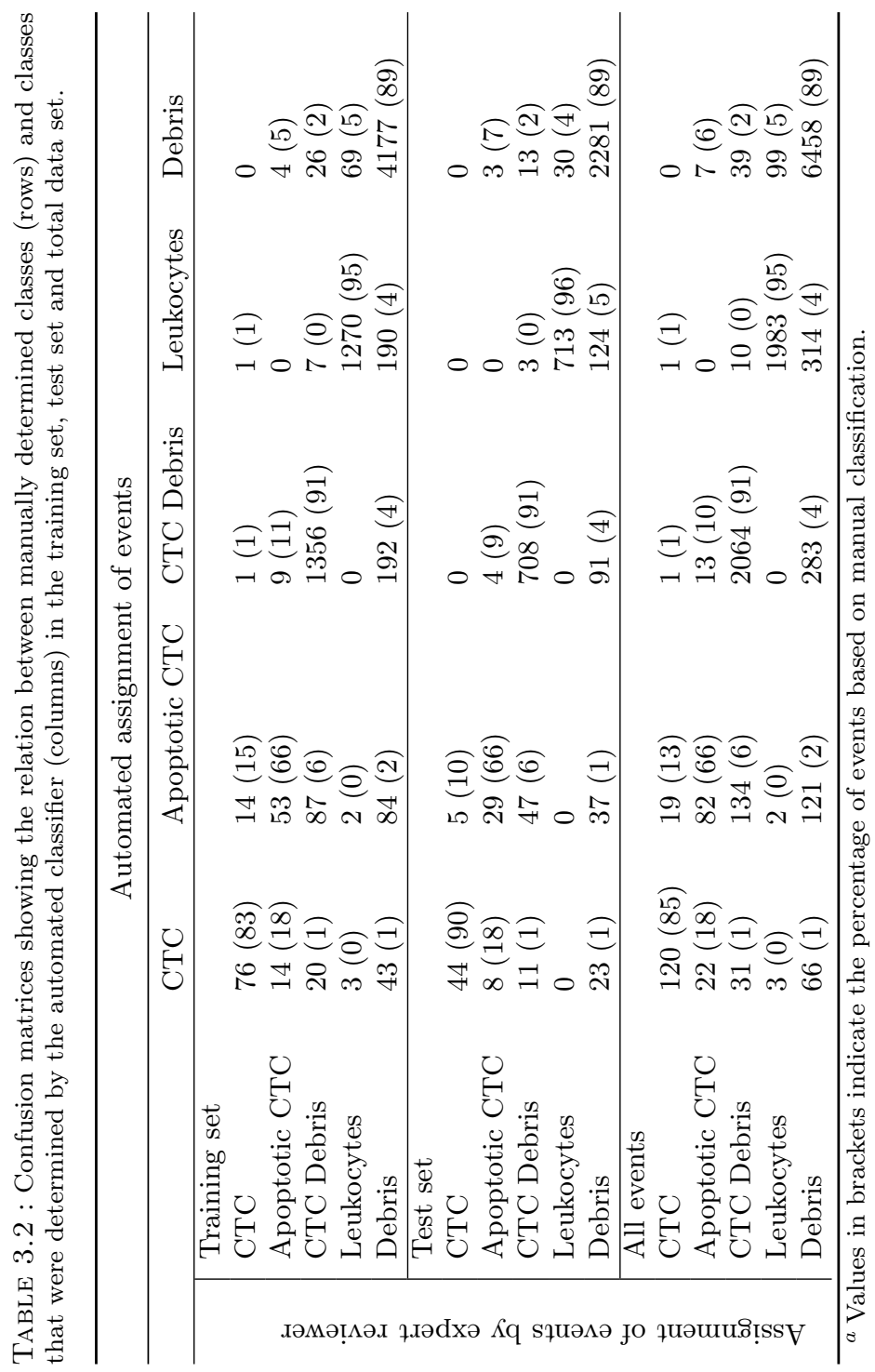



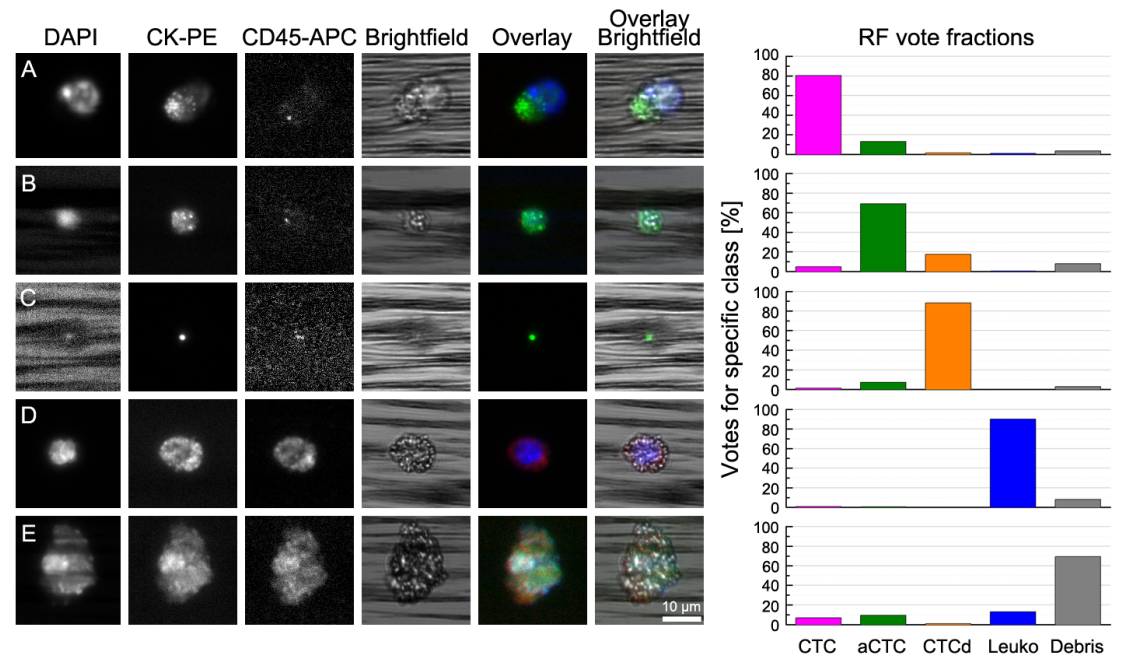

Figure 3.2 : CellTracks TDI images of the five event classes. Row A shows an intact CTC; Row B an apoptotic CTC; Row C CTC debris; Row D a leukocyte; Row E debris. The images show, from left to right, DAPI, CK-PE and CD45-APC fluorescence, bright-field, overlay of DAPI (blue), CK-PE (green) and CD45-APC (red) and the same overlay with the bright-field image added. The column chart to the right shows the distribution of votes for each class by the automated classification method for each of the 5 images. Colors in columns correspond to those in Figure 3.1. aCTC: apoptotic CTC and CTCd: CTC debris. Scale bar represents $10 \mu \mathrm{m}$.

intensity and Panel B shows the total CD45-APC intensity versus the mean DAPI intensity. These four parameters were the most important parameters during training of the automated classifier. The parameter on the y-axis of the graph in Panel A being the most important and the parameter on the $\mathrm{x}$-axis of Panel A being the second most important. Panel B shows the third and fourth most important parameters. Each dot represents an event that has passed the threshold. The analysis algorithm identified five classes of events that are represented by different colors. Magenta represents events classified as CTC, green represents events classified as apoptotic CTC, orange represents events classified as CTC debris, blue represents events classified as leukocytes and grey represents events classified as debris. The size of the dots varies between 3 and 9 pixels in diameter, depending on the margin with which that event was classified. The margin indicates the difference in the number of votes for the most likely and second most likely classes divided by the total number of votes. One can clearly see that events which are located away from the center of their respective clusters are smaller in size, denoting lower certainty in classification.

Figure 3.2 shows typical images from the five classes of events identified by the automated algorithm after they have passed the CK-PE threshold on 
the CellTracks TDI system. To the right of the images, a column chart is shown which indicates the percentage of votes that is assigned to each of the 5 classes for the corresponding event. Row A represents an event that has a high likelihood of being a CTC. The event has a clear nucleus (DAPI) and cytoskeleton (CK-PE), no signal for the leukocyte marker (CD45-APC) and a round to oval morphology (Bright-field). The composite of the nucleus (blue) and cytoskeleton (green) is shown in the overlay and the bright-field image is added to the composite in the adjacent image. The certainty of the event being classified as a CTC is $81 \%$ with a margin of $81 \%-13 \%$ $=68 \%$ compared to the next most likely apoptotic CTC class. Row B shows a typical apoptotic CTC with some nuclear material, punctuated cytokeratin staining, no staining with CD45-APC and a bright-field image showing a round-to-oval morphology. The typical cytokeratin staining can be attributed to the collapse of the cytoskeleton, which results in retraction of the cytokeratin filaments. The certainty of the event being classified as an apoptotic CTC is $69 \%$ with a margin of $52 \%$ compared to the next most likely CTC debris class. Row C shows CTC debris, with no staining of DAPI or CD45-APC, but clear staining with CK-PE. The diameter of this event is smaller than $4 \mu \mathrm{m}$ and it has a round appearance in the bright-field channel. The certainty of the event being classified as CTC debris is $88 \%$ with a margin of $81 \%$ compared to the next most likely apoptotic CTC class. Row D shows an event with a clear nucleus and staining with both CK-PE and CD45-APC. The bright-field image shows a round to oval morphology consistent with that of a leukocyte. The overlay images are consistent with that of a leukocyte non-specifically binding to CK-PE. The certainty of the event being classified as leukocyte is $90 \%$ with a margin of $82 \%$ compared to the next most likely debris class. Row E shows images associated with debris. These events frequently show similar staining in all 3 fluorescence channels. The certainty of the event being classified as debris is $69 \%$ with a margin of $56 \%$ compared to the next most likely leukocyte class.

\subsubsection{INTER AND INTRA OPERATOR VARIABILITY}

The automated classification of events by the Random Forest method is based on manual classification of those events by an expert reviewer. This expert reviewer uses several guidelines to determine the class of an event, like the presence of CD45-APC, size and intensity of the nucleus (DAPI), etc. Also, the current CellTracks Analyzer II uses operator based review of images of events to obtain a final CTC count. This method introduces uncertainty due to variations between multiple reviewers. A statistical study has shown that the operator variability is a major factor in consistent analysis of patient CTC samples [16].

To determine the inter reader variability and agreement on CellTracks TDI images, one thousand events were randomly selected from the entire database of 11872 events. These 1000 events were then presented to five 
TABLE 3.3 : Inter-reviewer agreement between five manual classifications and automated classification, using random forests, for a random selection of 1000 events

\begin{tabular}{llllll}
\hline & Rev. 2 & Rev. 3 & Rev. 4 & Rev. 5 & Auto \\
\hline Rev. 1 & $91.0 \%$ & $79.3 \%$ & $89.5 \%$ & $85.7 \%$ & $89.8 \%$ \\
Rev. 2 & & $79.6 \%$ & $90.4 \%$ & $87.4 \%$ & $87.9 \%$ \\
Rev. 3 & & & $80.4 \%$ & $77.4 \%$ & $77.6 \%$ \\
Rev. 4 & & & & $87.2 \%$ & $86.7 \%$ \\
Rev. 5 & & & & & $84.9 \%$ \\
\hline
\end{tabular}

${ }^{a}$ Rev. 1: Reviewer 1, Auto: Automated classifier

expert reviewers. They were asked to assign a class, 1 through 5 , to each event. Possible classes were the same as those used in the automated classification, namely: CTC, apoptotic CTC, CTC debris, leukocytes and debris. Every event had to be classified before the next event could be viewed. Table 3.3 shows the inter reviewer agreement between the five expert reviewers and their agreement with the automated classifier. The average agreement between the five expert reviewers is $84.8 \%$ with a standard deviation of $5.1 \%$. This means that, when presented with a random event, a second reviewer will classify the event the same as the first reviewer with an $\sim 85 \%$ chance. The intra reviewer agreement was determined in only one expert reviewer and was found to be $96.6 \%$. Second review of the same events was done on different days and after randomization of the data set to obtain a realistic value.

The agreement between the 5 reviewers and the automated classifier is on average $85.4 \%$ with a standard deviation of $4.7 \%$. Since there is no absolute definition of a correct class for each of the 1000 events, we used the class that occurred most frequently among the five reviewers as being the 'correct' class. Distribution of classification among reviewers was such that $67.9 \%$ of all events were agreed upon by all 5 reviewers (all 5 voted for the same class) and $21.5 \%$ of events were agreed upon by 4 out of 5 reviewers. Thus, $89.4 \%$ of all 1000 events were classified the same by at least 4 out of 5 reviewers. To determine if one class was more present in one category or the other, we compared the 894 events that were confidently agreed upon and the 106 events that were not. A ratio was determined that compares the fraction of a specific class in each of the two categories, effectively determining what class was most occurring in the events that were not readily agreed upon. These ratios are: CTC: 6.8 , apoptotic CTC: 12.7 , CTC debris: 1.3 , leukocytes: 1.0 and debris: 0.7 . This shows that reviewers disagreed the most on the classes CTC and apoptotic CTC. Further statistical analysis of the classes as assigned by the five reviewers was carried out [36-39]. Average agreement between reviewers is $84.8 \%$, however, some agreement between reviewers is to be expected based on chance. Kappa scores [39] were determined to illustrate the level of agreement between reviewers that 
is greater than chance and normalized to 1-chance. Overall kappa score is 0.75 , indicating a reasonable to good agreement between reviewers. The kappa scores were also calculated for each category separately and were: CTC: 0.55 , apoptotic CTC: 0.38, CTC debris: 0.86, leukocytes: 0.73 and debris: 0.74 . This confirms that the highest agreement between reviewers, above chance, is observed in CTC debris, leukocytes and debris.

\subsubsection{COMPARISON OF CTC ANALYSIS BY CELLTRACKS ANALYZER II AND CELLTRACKS TDI}

\section{Identification of leukocytes}

The efficiency of excitation of APC with a Mercury arc lamp, as used in the CellTracks Analyzer II, is less as compared to the $639 \mathrm{~nm}$ laser line used for APC excitation in the CellTracks TDI. This is illustrated by the signal intensity in the CD45-APC images in Figure 3.3. Blood samples from 9 healthy donors were prepared for CTC analysis. The sample cartridges were first analyzed by the CellTracks Analyzer II followed by analysis by the CellTracks TDI. Data analysis of the CellTracks Analyzer II was modified such that only CD45-APC positive and DAPI positive events were presented for review. An operator classified the events as leukocytes when the morphology of the events was consistent with that of a cell. Leukocytes in the CellTracks TDI were identified as CD45-APC positive and DAPI positive cells. The number of leukocytes identified by the CellTracks TDI ranged from 873-5245 (mean 1989 SD 1588) whereas the CellTracks Analyzer II identified only 286-3383 (mean 994 SD 1033) leukocytes in these samples. This corresponds to $30-80 \%$ (mean 47\%) of the leukocyte population identified by the CellTracks TDI.

\section{Identification of CTC}

To compare the two instruments, the same patient samples were analyzed on CellTracks Analyzer II and CellTracks TDI. Figure 3.4 shows images of the same 7 CTC acquired with the two different instruments. Images of the DAPI, CK-PE, CD45-APC and overlay of DAPI and CK-PE of each CTC are shown. In addition, a bright-field image is recorded and shown for CellTracks TDI. The detail in the images is clearly better in the CellTracks TDI images, as can be expected from the higher NA objective used in this instrument. Blood samples from 68 carcinoma patients and 9 healthy donors were prepared for CTC analysis by the CellTracks Autoprep and analyzed by the CellTracks Analyzer II. After analysis, the same sample cartridge was analyzed by the CellTracks TDI. Panel A of Figure 3.5 shows the comparison of the number of CTC detected by both instruments for the 68 patients. It shows a good correlation of $\mathrm{R}^{2}=0.87$ with a slope of 1.88 and an intercept of 0.2 . Bland-Altman data (not shown) reveals the interchangeability between both methods with a bias of 1.56 and $95 \%$ 


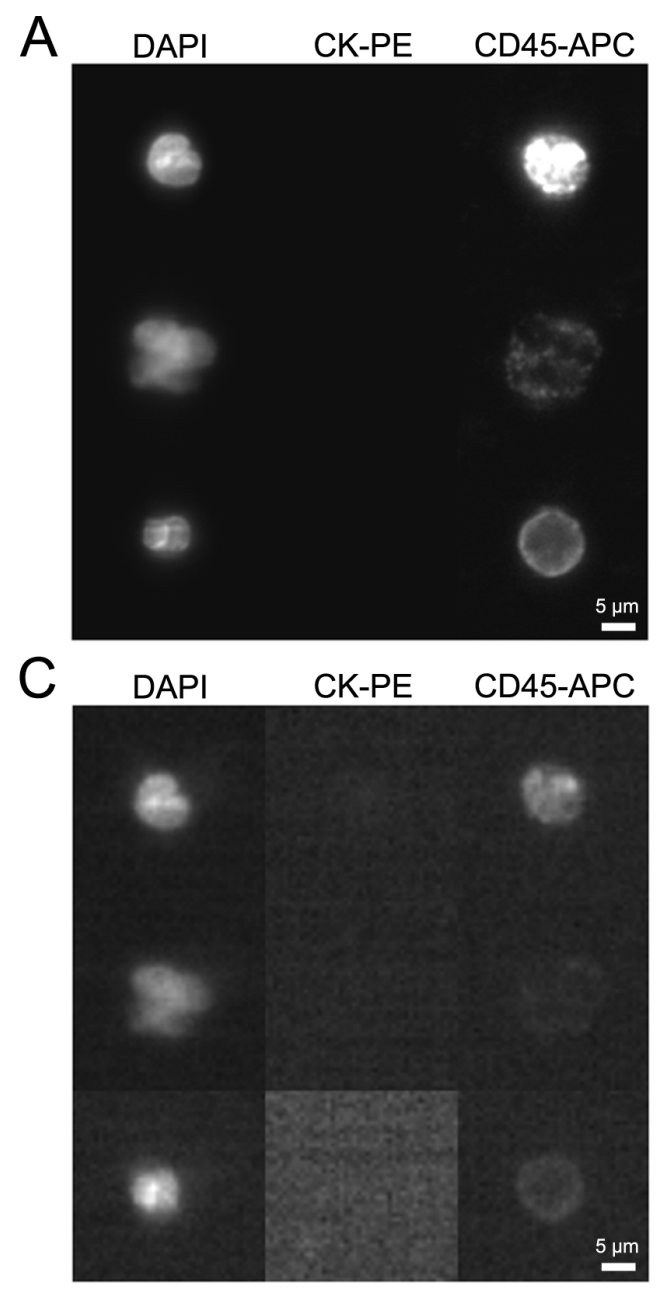

\section{B}
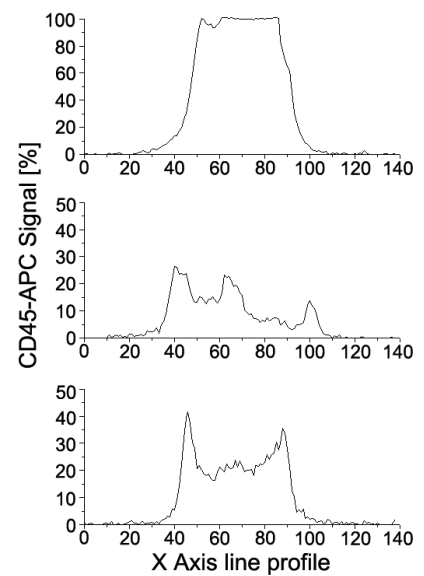

D

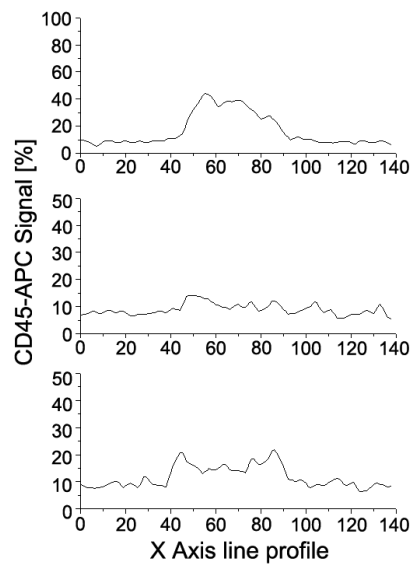

Figure 3.3: Comparison of images of leukocytes recorded with the CellTracks TDI and the CellTracks Analyzer II. (A) Three different cells analyzed by the CellTracks TDI. First column shows DAPI fluorescence, second column shows CK-PE fluorescence and the last column shows CD45-APC fluorescence. (B) Line profiles from the CD45-APC images in (A). (C) Same three cells now analyzed by the CellTracks Analyzer II. (D) Corresponding line profiles. Scale bar represents $5 \mu \mathrm{m}$. 


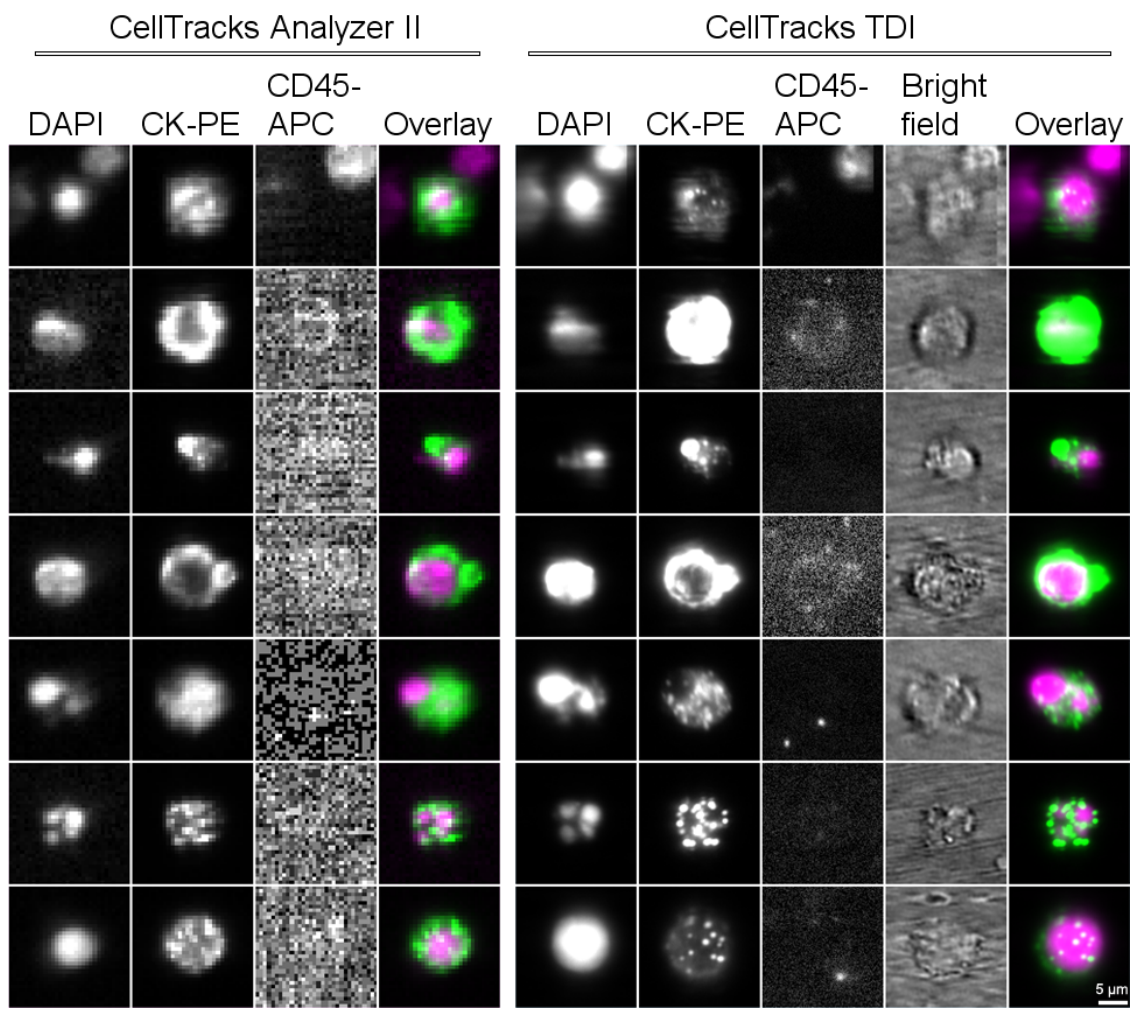

FIGURE 3.4 : Images of seven events classified as CTC or apoptotic CTC obtained with both CellTracks Analyzer II (left) and CellTracks TDI (right). Scale bar represents 5 $\mu \mathrm{m}$.

confidence levels in CTC counts of -9.5 and 6.4. Panel B of Figure 3.5 shows the relative occurrence of CTC, apoptotic CTC and CTC debris after automated classification of patient samples. The samples were sorted by decreasing number of CTC. In 17 (25\%) of the samples, CTC debris and or apoptotic CTC were detected when no intact CTC were detected.

\subsection{Discussion}

Presence of CTC in blood of patients with metastatic carcinomas is associated with poor survival prospects and their persistence after initiation of therapy strongly suggests an ineffective treatment [1-6]. A correct assessment of the presence of CTC is therefore of utmost importance. The prospective multicenter studies leading to these findings were made possible through the use of standardized CTC enumeration by the CellSearch system. In this system, CTC classification relies on the final selection by 
A

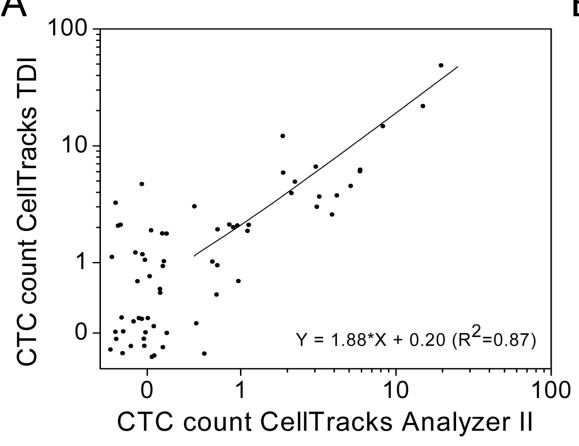

B

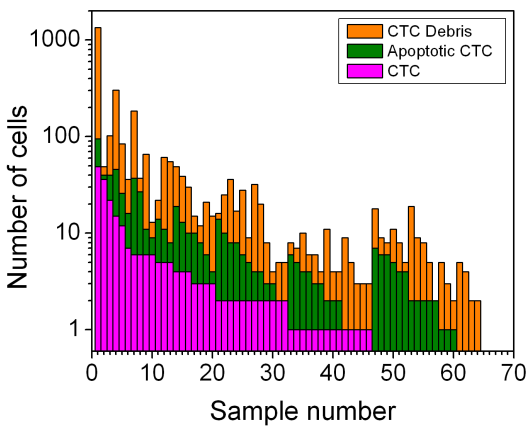

Figure 3.5 : (A) Comparison of CTC counts obtained with CellTracks Analyzer II and the automated classification of CTC with the CellTracks TDI and (B) the variation in CTC count, apoptotic CTC count and CTC debris count by the automated classification with the CellTracks TDI across 68 patient samples. Data points in (A) were jittered by up to half a count and both axis have combined linear and logarithmic scales, changing between 0 and 1 .

an operator on a set of computer pre-selected events based on cytokeratin and DNA content. Variability of CTC classification by different operators has been identified as the main source of error and a likely explanation that more than 1 CTC has to be detected before a sample is considered CTC positive. In the CellTracks Analyzer II, images presented to the operator are auto-scaled to increase visibility. However, the human eye can only discern approximately 100 gray levels, making the deduction of quantitative information by the operator from such images difficult.

In auto-scaled images the relation between "true" signal and background is less apparent. A dim nucleus may appear to be just as bright as a brighter nucleus and a slight difference in monitor settings may already result in a different interpretation by the operator. The large heterogeneity of the morphological appearance of CTC further contributes to the variation in classification of objects as CTC. In this study, CellTracks TDI was developed to automate the classification of events based on Random Forest classification using several types of parameters. The major differences between the CellTracks Analyzer II and CellTracks TDI are the objective, $10 \times / 0.45 \mathrm{NA}$ versus $40 \times / 0.6 \mathrm{NA}$, the use of a mercury arc lamp versus laser lines and a regular CCD camera versus a TDI camera. These differences result in a higher resolution and an increase in sensitivity. The higher sensitivity for APC resulted in a higher efficiency of the detection of leukocytes identified by CD45-APC by CellTracks TDI. The lower efficiency of leukocyte detection by the CellTracks Analyzer II can most likely be contributed to granulocytes that express the CD45 antigen at lesser density. The higher APC sensitivity of the CellTracks TDI results in the ability to identify DAPI positive, CKPE negative, CD45-APC dim intact cells. CTC that express EpCAM, but 
lack cytokeratin 8,18 and 19 will be among these cells. CellTracks TDI can be used to investigate the frequency of such cells in cancer patients and to verify whether or not they are indeed CTC by detection of chromosomal abnormalities [19, 40, 41].

CTC are extremely rare and accurate detection of a few CTC in $7.5 \mathrm{ml}$ of blood is strongly dependent on the Poisson distribution and the assay variability. Statistical calculations [16] have shown that 1 or more CTC, at $95 \%$ confidence, are detected in a $7.5 \mathrm{ml}$ blood sample, after processing using CellSearch, for in-vivo concentrations down to $2600 \mathrm{CTC}$ in $5 \mathrm{~L}$ of blood. This amounts to a concentration of $\sim 0.5 \mathrm{CTC} / \mathrm{ml}$. Classifying objects as CTC was found to be the largest contributor of errors in CTC determination and the automated CTC classification presented here can help to reduce this error. The CellSearch system recovers $85 \%$ of cells from the tumor cell line SKBR-3 spiked in $7.5 \mathrm{ml}$ of blood [14]. The specificity of the assay is $99.7 \%$ for healthy subjects and patients with nonmalignant disease. In healthy subjects, only 8 of 145 samples $(5.5 \%)$ were found to have 1 CTC per $7.5 \mathrm{ml}$ of blood and in all others no CTC were detected [14].

The random forest method that was used for automated classification of events proved to be versatile. It can for example be used to determine an outlier measure of events based on the original manual review of events. This could further improve classification in future measurements because the manual classification, which is the basis for automated classification, can be further optimized. Also, the parameters that are used in the classification can be optimized by determining their importance. Some parameters that were in the original set as used in this article proved to be of little to no importance in classification. Some further initial experiments indicate that these parameters can be left out of the data set without any significant detrimental effect on classification accuracy but reducing the time it took to train the classifier.

General classification accuracy of the automated classifier proved to be good with excellent recall rates for all classes except apoptotic CTC, which was only $66 \%$. Precision of classification was excellent for CTC debris, leukocytes and debris, mediocre (0.50) for CTC and low for apoptotic CTC. The lower precision of classification for CTC and apoptotic CTC is mainly due to the false positive events which are, according to manual review, debris but are not classified as such. Although the percentage of these events is low, their relative number as compared to the total number of e.g. CTC is high due to the large number of debris events present in the patient samples. Further improvements to the CellTracks TDI system might therefore come from reducing the number of debris events in the patient samples after preparation. Also, the automated classifier could possibly be trained to prevent false positive classification of events as CTC or apoptotic CTC. However, we do not know in what way this will affect the recall rate of the classifier, which is currently $90.4 \%$ over the entire dataset.

Inter reviewer variability and agreement results indicate that the au- 
tomated classifier has similar agreement to the expert reviewer that was used for classification of the entire dataset as compared to the five reviewers among each other. Also, the intra reviewer agreement was shown to be $96.6 \%$, whereas that of the automated classifier is of course $100 \%$. These results indicate that the automated classification of events in the CellTracks TDI by the random forest method is comparable to a human expert reviewer while being always consistent. This method could therefore be used to standardize classification of CTC in the CellTracks system. Provided however that separate instruments have similar output values with regards to e.g. fluorescence intensity or at least a way to measure and process the difference between them and standardize the output values.

Results from Figure $3.5 \mathrm{~A}$ indicate that in $25 \%$ of patient samples, CTC debris was present without the presence of CTC. Destruction of intact CTC by mechanical stress during the immunomagnetic enrichment could lead to CTC debris. This is however unlikely as such phenomenona are not observed when cells from tumor cell lines spiked in blood are processed. Moreover, CTC debris can be observed in patients with large numbers of CTC that have not been immunomagnetically enriched [42]. The presence of apoptotic bodies near the tumor site and in the blood stream are reported by others [17, 43-46] as well as an increase of cytokeratin 18, 19 and caspase cleaved cytokeratin in serum of patients with carcinomas [47-52] supporting the notion that the detected tumor debris is indeed directly related to the presence of a tumor. A recent retrospective analysis of $\mathrm{EpCAM}^{+} \mathrm{CD} 45^{-} \mathrm{CK}^{+}$ objects detected with the CellSearch system in the prospective multi center CTC study of castration-resistant prostate cancer showed that the smaller CTC related objects also correlated to poor survival [13]. Only CTC that express EpCAM as well as Cytokeratin 8, 18 or 19 will be detected by this system. The frequency and the relation to clinical outcome of CTC that do not express these antigens is being explored through a variety of alternative approaches for the detection of CTC [9, 10, 53-58].

Although only viable CTC will have the potential to form metastasis, the presence of apoptotic CTC or CTC debris might also be an indicator of a worse prognosis. However, this will have to be uncovered in controlled clinical studies. CTC debris events occur more frequently than intact CTC and can therefore be enumerated with a smaller statistical error. The discrimination between the different CTC populations may also be of value for determination of therapy effectiveness. Shortly after administration of a therapy, a decrease in intact cells and an increase in cell debris might suggest an effective therapy.

\subsection{ACKNOWLEDGEMENTS}

We thank Dr. M. de Groot from MST hospital in Enschede, The Netherlands, for kindly supplying blood specimens from the patients. 


\subsection{REFERENCES}

[1] M. Cristofanilli, T. Budd, M. Ellis, A. Stopeck, J. Matera, M. Miller, J. Reuben, G. Doyle, W. Allard, L. Terstappen, and D. Hayes, "Circulating tumor cells, disease progression, and survival in metastatic breast cancer," N Engl J Med, vol. 351(8), pp. 781-791, 2004.

[2] M. Liu, P. Shields, R. Warren, P. Cohen, M. Wilkinson, Y. Ottaviano, S. Rao, J. Eng-Wong, F. Seillier-Moiseiwitsch, A. Noone, and C. Isaacs, "Circulating tumor cells: A useful predictor of treatment efficacy in metastatic breast cancer," Journal of Clinical Oncology, vol. 27(31), pp. 5153-5159, 2009.

[3] S. Cohen, C. Punt, N. Iannotti, B. Saidman, K. Sabbath, N. Gabrail, J. Picus, M. Morse, E. Mitchell, C. Desch, M. Miller, G. Doyle, H. Tissing, L. Terstappen, and N. Meropol, "The relationship of circulating tumor cells to tumor response, progression-free survival, and overall survival in patients with metastatic colorectal cancer," Journal of Clinical Oncology, vol. 26(19), pp. 3213-3221, 2008.

[4] J. Tol, M. Koopman, M. Miller, A. Tibbe, A. Cats, G. Creemers, A. Vos, I. Nagtegaal, L. Terstappen, and C. Punt, "Circulating tumour cells early predict progression-free and overall survival in advanced colorectal cancer patients treated with chemotherapy and targeted agents," Annals of Oncology, vol. 21(5), pp. 1006-1012, 2010.

[5] J. De Bono, H. Scher, R. Montgomery, C. Parker, M. Miller, H. Tissing, G. Doyle, L. Terstappen, K. Pienta, and D. Raghavan, "Circulating tumor cells predict survival benefit from treatment in metastatic castration resistant prostate cancer," Clin Can Res, vol. 14(19), pp. 6302-6309, 2008.

[6] D. Olmos, H. Arkenau, J. Ang, I. Ledaki, G. Attard, C. Carden, A. Reid, R. Hem, P. Fong, N. Oomen, R. Molife, D. Dearnaley, C. Parker, L. Terstappen, and J. DeBono, "Circulating tumor cells (ctc) as intermediate end points in castrationresistant prostate cancer (crpc): a single-centre experience," Annals of Oncology, vol. 20, pp. 27-37, 2009.

[7] S. Nagrath, L. Sequist, S. Maheswaran, D. Bell, D. Irimia, L. Ulkus, M. Smith, E. Kwak, S. Digumarthy, A. Muzikansky, P. Ryan, U. Balis, R. Tompkins, D. Haber, and M. Toner, "Isolation of rare circulating tumour cells in cancer patients by microchip technology," Nature, vol. 450, pp. 1235-1241, 2007.

[8] K. Pachmann, O. Camara, A. Kavallaris, S. Krauspe, N. Malarski, M. Gajda, T. Kroll, C. Jörke, U. Hammer, A. Altendorf-Hofmann, C. Rabenstein, U. Pachmann, I. Runnebaum, and K. Höffken, "Monitoring the response of circulating epithelial tumor cells to adjuvant chemotherapy in breast cancer allows detection of patients at risk of early relapse," JCO, vol. 26, pp. 1208-1215, 2008.

[9] H. Kahn, A. Presta, L. Yang, J. Blondal, M. Trudeau, L. Lickley, C. Holloway, D. McCready, D. Maclean, and A. Marks, "Enumeration of circulating tumor cells in the blood of breast cancer patients after filtration enrichment: correlation with disease stage," Breast Cancer Res Treat, vol. 86, pp. 237-247, 2004.

[10] R. Krivacic, A. Ladanyi, D. Curry, H. Hsieh, P. Kuhn, D. Bergsrud, J. Kepros, T. Barbera, M. Ho, L. Chen, R. Lerner, and R. Bruce, "A rare-cell detector for cancer," PNAS, vol. 101, pp. 10501-10504, 2004.

[11] H. Hsieh, D. Marrinucci, K. Bethel, D. Curry, M. Humphrey, R. Krivacic, J. Kroener, L. Kroener, A. Ladanyi, N. Lazarus, P. Kuhn, R. Bruce, and N. J, "High speed detection of circulating tumor cells," Biosens Bioelectron, vol. 21, pp. 1893-1899, 2006.

[12] E. Racila, D. Euhus, A. Weiss, C. Rao, J. McConnell, L. Terstappen, and J. Uhr, "Detection and characterization of carcinoma cells in the blood," PNAS, vol. 95, pp. 4589-4594, 1998. 
[13] F. Coumans, C. Doggen, G. Attard, J. de Bono, and L. Terstappen, "All circulating $\mathrm{EpCAM}+\mathrm{CD} 45-\mathrm{CK}+$ but not EpCAM $+\mathrm{CD} 45+\mathrm{CK}+$ objects predict overall survival in castration-resistant prostate cancer," Annals of Oncology, vol. 21(9), pp. 1851$1857,2010$.

[14] W. Allard, J. Matera, M. Miller, M. Repollet, M. Connelly, C. Rao, A. Tibbe, J. Uhr, and L. Terstappen, "Tumor cells circulate in the peripheral blood of all major carcinomas but not in healthy subjects or patients with nonmalignant diseases," Clinical Cancer Research, vol. 10(20), pp. 6897-6904, 2004.

[15] M. Kagan, D. Howard, T. Bendele, J. Mayes, J. Silvia, M. Repollet, J. Doyle, J. Allard, N. Tu, T. Bui, T. Russell, C. Rao, M. Hermann, H. Rutner, and L. Terstappen, "A sample preparation and analysis system for identification of circulating tumor cells," J Clinical Ligand Assay, vol. 25(1), pp. 104-110, 2002.

[16] A. Tibbe, C. Miller, and L. Terstappen, "Statistical considerations for enumeration of circulating tumor cells," Cytometry Part A, vol. 71A, pp. 154-162, 2007.

[17] C. Larson, J. Moreno, K. Pienta, S. Gross, M. Repollet, S. OHara, T. Russell, and L. Terstappen, "Apoptosis of circulating tumor cells in prostate cancer patients," Cytometry part A, vol. 62(1), pp. 46-53, 2004.

[18] T. Scholtens, F. Schreuder, S. Ligthart, J. Swennenhuis, A. Tibbe, J. Greve, and L. Terstappen, "Celltracks TDI: An image cytometer for cell characterization," Cytometry Part A, vol. 79(3), pp. 203-213, 2011.

[19] F. Schreuder, Laser image cytometer for analysis of circulating tumor cells. PhD thesis, PhD Thesis University of Twente, ISBN 9789036526104, 2008.

[20] A. Tibbe, B. de Grooth, J. Greve, G. Dolan, C. Rao, and L. Terstappen, "Magnetic field design for selecting and aligning immunomagnetic labeled cells," Cytometry, vol. 47(3), pp. 163-172, 2002.

[21] W. Ortyn, B. Hall, T. George, K. Frost, D. Basiji, D. Perry, C. Zimmerman, D. Coder, J. Philip, and P. Morrissey, "Sensitivity measurement and compensation in spectral imaging," Cytometry Part A, vol. 69(8), pp. 852-862, 2006.

[22] W. Rasband, "Imagej," http://rsbweb.nih.gov/ij/index.html, vol. -, pp. -, 1997 2010.

[23] M. Abramoff, P. Magelhaes, and S. Ram, "Image processing with ImageJ," Biophotonics International, vol. 11(7), pp. 36-42, 2004.

[24] P. Liao, T. Chen, and P. Chung, "A fast algorithm for multilevel thresholding," Journal of Information Science and Engineering, vol. 17(5), pp. 713-727, 2001.

[25] L. Breiman, "Bagging predictors," Mach Learning, vol. 24(2), pp. 123-140, 1996.

[26] L. Breiman, "Random forests," Mach Learning, vol. 45(1), pp. 5-32, 2001.

[27] B. Wu, T. Abbott, D. Fishman, W. McMurray, G. Mor, K. Stone, D. Ward, K. Williams, and H. Zhao, "Comparison of statistical methods for classification of ovarian cancer using mass spectrometry data," Bioinformatics, vol. 19(13), pp. 1636$1643,2003$.

[28] P. Gislason, J. Benediktsson, and J. Sveinsson, "Random forests for land cover classification," Pattern Recog Lett, vol. 27(4), pp. 294-300, 2006.

[29] T. Ho, "The random subspace method for constructing decision forests," IEEE Trans Pattern Anal Mach Intell, vol. 20(8), pp. 832-844, 1998.

[30] J. Ham, Y. Chen, M. Crawford, and J. Ghosh, "Investigation of the random forest framework for classification of hyperspectral data," IEEE Trans Geosci Remote Sens, vol. 43(3), pp. 492-501, 2005. 
[31] W. Geng, P. Cosman, C. Berry, Z. Feng, and W. Schafer, "Automatic tracking, feature extraction and classification of c. elegans phenotypes," IEEE Transactions on Biomedical Engineering, vol. 51(10), pp. 1811-1820, 2004.

[32] J. Ramírez, J. Górriz, R. Chaves, M. López, D. Salas-Gonzalez, I. Álvarez, and F. Segovia, "Spect image classification using random forests," Electron Lett, vol. 45(12), pp. 604-605, 2009.

[33] V. Kovalev, N. Harder, B. Neumann, M. Held, U. Liebel, H. Erfle, J. Ellenberg, R. Eils, and K. Rohr, "Feature selection for evaluating fluorescence microscopy images in genome-wide cell screens," Proceedings of the IEEE computer society conference on computer vision and pattern recognition, vol. -, pp. 276-283, 2006.

[34] J. Newberg, J. Li, A. Rao, F. Ponten, M. Uhlen, E. Lundberg, and R. Murphy, "Automated analysis of human protein atlas immunofluorescence images," IEEE int symp on biomedical imaging, vol. -, pp. 1023-1026, 2009.

[35] T. Khoshgoftaar, M. Golawala, and J. Van Hulse, "An empirical study of learning from imbalanced data using random forest," 19th IEEE International conference on tools with artificial intelligence, vol. -, pp. 310-317, 2007.

[36] J. Landis and G. Koch, "The measurement of observer agreement for categorical data," Biometrics, vol. 33(1), pp. 159-174, 1977.

[37] J. Cohen, "Weighted kappa: Nominal scale agreement provision for scaled disagreement or partial credit," Psychological bulletin, vol. 70(4), pp. 213-220, 1968.

[38] J. Sim and C. Wright, "The kappa statistic in reliability studies: Use, interpretation, and sample size requirements," Physical Therapy, vol. 85(3), pp. 257-268, 2005.

[39] J. Fleiss, "Measuring nominal scale agreement among many raters," Psychological bulletin, vol. 76(5), pp. 378-382, 1971.

[40] G. Attard, J. Swennenhuis, D. Olmos, A. Reid, E. Vickers, R. Hern, R. Levink, F. Coumans, J. Moreira, R. Riisnaes, N. Oomen, G. Hawche, C. Jameson, E. Thompson, R. Sipkema, C. Carden, C. Parker, D. Dearnaley, S. Kaye, C. Cooper, A. Molina, M. Cox, L. Terstappen, and J. de Bono, "Characterization of erg, ar and pten status in circulating tumor cells from patients with castration-resistant prostate cancer," Cancer Research, vol. 69, pp. 2912-2918, 2009.

[41] J. Swennenhuis, A. Tibbe, R. Levink, R. Sipkema, and L. Terstappen, "Characterization of circulating tumor cells by fluorescence in-situ hybridization," Cytometry Part A, vol. 75(6), pp. 520-527, 2009.

[42] C. Rao, D. Chianese, G. Doyle, M. Miller, T. Russell, R. Sanders, and L. Terstappen, "Expression of epithelial cell adhesion molecule in carcinoma cells present in blood and primary and metastatic tumors," Int J Oncology, vol. 27(1), pp. 49-57, 2005.

[43] M. Leers, V. Björklund, B. Björklund, H. Jörnvall, and M. Nap, "An immunohistochemical study of the clearance of apoptotic cellular fragments," Cell Mol Life Sci, vol. 59, pp. 1358-1365, 2002.

[44] N. Ku, J. Liao, and M. Omary, "Apoptosis generates stable fragments of human type i keratins," J of Bio Chem, vol. 272, pp. 33197-33203, 1997.

[45] Z. Wang, M. Eisengberger, M. Carducci, A. Partin, H. Scher, and P. TsŠo, "Identification and characterization of circulating prostate carcinoma cells," Cancer, vol. 88, pp. 2787-2795, 2000.

[46] G. Mehes, A. Witt, E. Kubista, and P. Ambros, "Circulating breast cancer cells are frequently apoptotic," Am J Pathol, vol. 159, pp. 17-20, 2001. 
[47] M. Hägg, K. Biven, T. Ueno, L. Rydlander, P. Björklund, K. Wiman, M. Shoshan, and S. Linder, "A novel high throughput assay for screening of pro-apoptotic drugs," Investigational New Drugs, vol. 20, pp. 253-259, 2002.

[48] B. Schutte, M. Henfling, W. Kolgen, M. Bouman, S. Meex, M. Leers, M. Nap, V. Björklund, P. Björklund, B. Björklund, E. Lane, M. Omary, H. Jörnvall, and F. Ramaekers, "Keratin $8 / 18$ breakdown and reorganization during apoptosis," Experimental Cell Research, vol. 297, pp. 11-26, 2004.

[49] G. Kramer, H. Erdal, H. Mertens, M. Nap, J. Mauermann, G. Steiner, M. Marberger, K. Biven, M. Shoshan, and S. Linder, "Differentiation between cell death modes using measurements of different soluble forms of extracellular cytokeratin 18," Cancer Res, vol. 64, pp. 1751-1756, 2004.

[50] S. Jahr, H. Hentze, S. Englisch, D. Hardt, F. Fackelmayer, R. Hesch, and R. Knippers, "Dna fragments in the blood plasma of cancer patients: Quantitations and evidence for their origin from apoptotic and necrotic cells," Can Res, vol. 61, pp. 1659-1665, 2001.

[51] M. Olofsson, T. Ueno, Y. Pan, R. Xu, F. Cai, H. van der Kuip, T. Muerdter, M. Sonnenberg, W. Aulitzky, S. Schwarz, E. Andersson, M. Shoshan, A. Havelka, M. Toi, and S. Linder, "Cytokeratin-18 is a useful serum biomarker for early determination of response of breast carcinomas to chemotherapy," Clin Cancer Res, vol. 11, pp. 3198-3206, 2007.

[52] R. Lai, H. Hsu, J. Lu, L. Ger, and N. Lai, "Cyfra 21-1 enzyme-linked immunosorbent assay evaluation as a tumor marker in non-small cell lung cancer," Chest, vol. 109, pp. 995-1000, 1996.

[53] C. Alix-Panabieres, J. Brouillet, M. Fabbro, H. Yssel, T. Rousset, T. Maudelonde, G. Choquet-Kastylevsky, and J. Vendrell, "Characterization and enumeration of cells secreting tumor markers in the peripheral blood of breast cancer patients," $J$ Immunol Methods, vol. 299, pp. 177-188, 2005.

[54] G. Vona, A. Sabile, M. Louha, V. Sitruk, S. Romana, K. Schutze, F. Capron, D. Franco, M. Pazzagli, M. Vekemans, B. Lacour, C. Brechot, and P. PaterliniBrechot, "Isolation by size of epithelial tumor cells: A new method for the immunomorphological and molecular characterization of circulating tumor cells," $\mathrm{Am}$ J Pathol, vol. 156(1), pp. 57-63, 2000.

[55] J. Gleghorn, E. Pratt, D. Denning, H. Liu, N. Bander, S. Tagawa, D. Nanus, P. Giannakakou, and B. Kirby, "Capture of circulating tumor cells from whole blood of prostate cancer patients using geometrically enhanced differential immunocapture (gedi) and a prostate-specific antibody," Lab on a Chip, vol. 10(1), pp. 27-29, 2010.

[56] R. Königsberg, E. Obermayr, G. Bises, G. Pfeiler, M. Gneist, F. Wrba, M. De Santis, R. Zeillinger, M. Hudec, and C. Dittrich, "Detection of epcam positive and negative circulating tumor cells in metastatic breast cancer patients," Acta Oncol, vol. 50(5), pp. 700-710, 2011.

[57] P. Gascoyne, J. Noshari, T. Anderson, and F. Becker, "Isolation of rare cells from cell mixtures by dielectrophoresis," Electrophoresis, vol. 30(8), pp. 1388-1398, 2009.

[58] S. Tan, R. Lakshmi, P. Chen, W. Lim, L. Yobas, and C. Lim, "Versatile label free biochip for the detection of circulating tumor cells from peripheral blood in cancer patients," Biosensors and Bioelectronics, vol. 26(4), pp. 1701-1705, 2010. 


\section{DEVELOPMENT AND CHARACTERIZATION OF CELL ALIGNMENT STRUCTURES}

To improve the characterization and augment the analysis speed of Circulating Tumor Cells (CTC), we have developed cell alignment structures for use in an image cytometer. These structures mechanically concentrate magnetically labeled cells onto an analysis surface, reducing the area that needs to be imaged.

Four different types of cell alignment microstructures were designed, developed and fabricated using cleanroom technologies: anisotropic $(\mathrm{KOH})$ etching, reactive ion etching (RIE) and deep reactive ion etching (DRIE). PDMS micro molding was used to create imprints from the wafers that contain the negative structures. These imprints were cut to size and glued to the upper side of cartridges that contain the samples during the analysis. A Magnest ${ }^{\mathrm{TM}}$ Cell Presentation Device is then used to force the magnetically labeled cells up to this analysis surface, where they align in predetermined areas. The microstructures were evaluated based on the results obtained with cell samples. Points considered are: image quality, capture efficiency, alignment efficiency, fluorescence detection efficiency and suitability for implementation in the CellTracks TDI system.

The four types of cell alignment microstructures that were designed and tested are: 1. V-Groove structure that aligns the cells in long lines of single cells like in a 'frozen' flow cytometry stream. The sharp edges of the $\mathrm{V}$ grooves cause some image distortion. 2. Flat-top V-groove structure that aligns the cells in 8 to $17 \mu \mathrm{m}$ wide strips. The flat upper surface 
results in better image quality. 3. Chimney structure that collects unbound magnetic (ferrofluid) labels away from the labeled cells. This minimizes the distortive effect of unbound magnetic label on imaging of the cells. 4 . U-Groove structure that aligns the cells in six, $80 \mu \mathrm{m}$ wide, channels to reduce the effective imaging area. The U-Groove yields the best image quality, alignment efficiency and fluorescence detection efficiency of the tested structures. Images are comparable to those obtained using a flat glass surface.

The developed cell alignment microstructures in PDMS may be used to concentrate cells along lines and strips, thereby greatly reducing the time needed to analyze a sample. The U-Groove structure is the most suitable one for implementation into the CellTracks TDI system.

\subsection{INTRODUCTION}

In recent years, image cytometry has seen an increase in applications for rare cell analysis and characterization. An image cytometer usually consists of an automated fluorescence microscope equipped with a high resolution objective, a high power light source, motorized stages and computer automation. The cells reside at an analysis surface which is usually a microscope slide or a cartridge. Often one or more lasers are used to illuminate the cells for fluorescence excitation. The analysis surface is mechanically or optically scanned and signal detection is performed by a PMT or a CCD camera, using frame transfer mode. The images are captured and evaluated by image analysis software. Advantages of this analysis method as compared to flow cytometry, which is most widely used in clinical settings, are the extensive morphological information obtained and the ability to revisit cells of interest. The main disadvantage of image cytometry for rare cell analysis is the much lower throughput and therefore generally longer time required to obtain reliable statistics and needed specificity. This is due to the fact that at the analysis surface the (rare) cells are spread out randomly, and the greater part of the available area does not contain any cell. Yet the whole surface area has to be scanned. To decrease the imaging time and increase the throughput, the area where the cells are positioned should be reduced while maintaining the same sample volume and specificity.

Several methods have been developed in the past to either reduce the analysis surface that needs to be scanned or align the cells into predefined areas. One early simple system is the Cytodisk, developed by de Grooth et al [1]. It uses a flat disk or plate equipped with circular V-shaped grooves. Cell suspensions are spread out on the grooved surface and the cells sediment to the bottom of the groove. Then the suspension is dried out and the grooves are scanned using a fiber system attached to a record player. A more recent method is the CellTracks system [2-6]. It employs an analysis cartridge of which the upper surface is covered by thin, $15 \mu \mathrm{m}$ wide, 
parallel ferromagnetic nickel lines at a distance of $15 \mu \mathrm{m}$. The cartridge is placed inside a magnetic yoke. The field gradient forces ferromagnetic labeled cells to move to the upper surface. The nickel lines help to shape the field gradient in such a way that the cells end up between the lines. A scanning system based on CD player technology is then used to record fluorescence images of the cells. With the original system a 3-color leukocyte differentiation can be performed.

In this chapter we discuss novel methods to reduce the analysis surface area in an image cytometer. Basically we combined the two principles mentioned above. We developed microstructures to concentrate labeled cells by magnetic force into lines and strips at the surface. Several differently shaped microstructures were manufactured and tested. The microstructures need very smooth surfaces to ensure optimal mechanical guiding of the labeled cells into the channels or grooves. This is especially important when a sloped sidewall exists, where the cells are in direct contact with the wall and have to slide along it to reach the final destination at the surface. If the sidewalls are not smooth enough, cells may be trapped before they reach the imaging surface. This will lower the capture efficiency of that structure.

We have used anisotropic wet- and dry etching techniques to fabricate the designed microstructures using cleanroom technology. The negative of the desired structure was etched in a silicon wafer. Next, we used PDMS imprinting to generate positive structures. PDMS was chosen because of its unique material properties which make the material very well suited for making imprints. Four different structures were tested and evaluated.

\subsection{Materials And Methods}

\subsubsection{DESIGN OF CELL ALIGNMENT MiCROSTRUCTURES}

The first microstructure, also called V-Groove, was designed to align the cells in long lines, like in flow cytometry, and offer unobstructed imaging. By aligning the cells in long lines, they could be scanned using the laser spot scanning system that was in use at the time [2]. The layout of the structure as well as the main design parameters are shown in Figure 4.1A.

The angle, $70.52^{\circ}$, indicated in the figure results from the anisotropic wet etching technique that is used during fabrication and is the same for all structures. The distance marked by $\mathrm{D}$ indicates the spacing of the $\mathrm{V}$-shaped grooves. The spacing can be adapted to the number of cells that are expected to be in the sample. To accommodate a range of cell concentrations, a wafer was designed that contained 3 values for $\mathrm{D}$, as shown in Table 4.1. The total number of V-Grooves present on the analysis surface can be calculated by dividing the width of the analysis surface by the spacing of the V-Grooves. The width of the analysis surface is $2.7 \mathrm{~mm}$ and the spacing of the V-Grooves is 30,150 or $300 \mu \mathrm{m}$, giving a total number of grooves of 90,18 and 9 respectively. Since the length of each groove is 30 

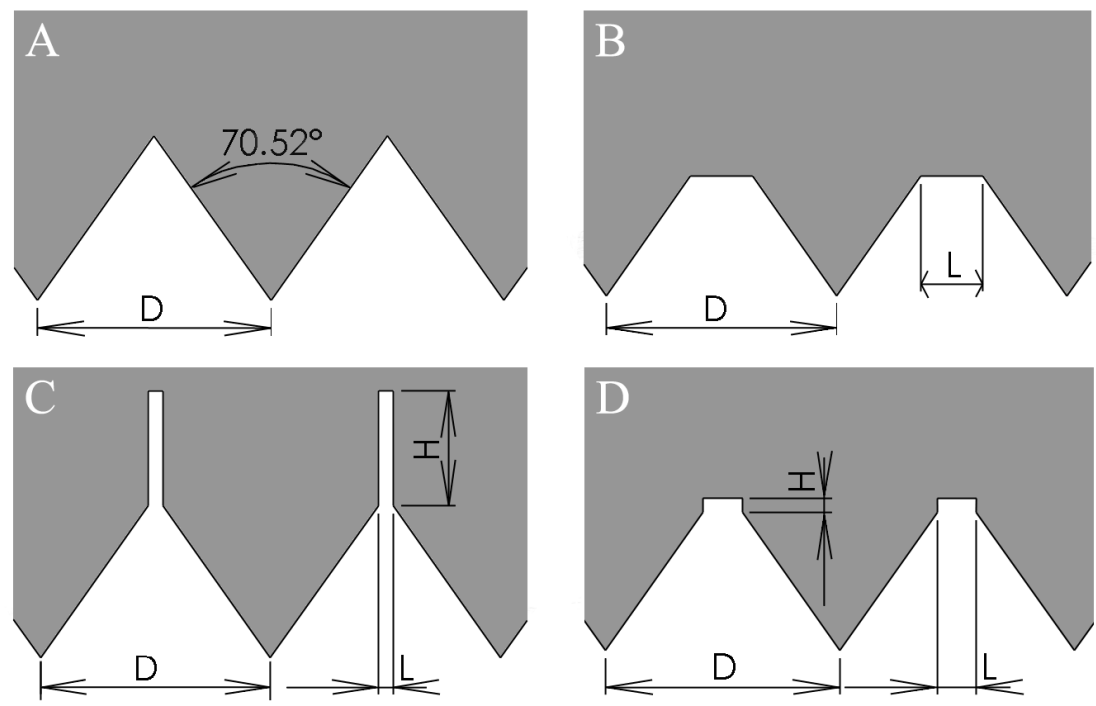

FIGURE 4.1 : Schematic overview image of the four microstructures that were developed and are discussed in this chapter. The gray area in all four images indicates PDMS. Cells are below the PDMS structure when they are first inserted into the analysis chamber. Due to the magnetic force exerted on the cells by the external magnetic field, the cells start to move upwards towards the structures where they are mechanically forced into the correct location, in preparation for scanning. (A) V-Grooved microstructure; this structure was developed to both align and concentrate the cells in long lines, as in Flow Cytometry (FCM). (B) Flat-top V-Groove microstructure; this structure was developed to also align the cells in long lines, as in FCM, but it features a flat area at the top to allow for better image quality. (C) Chimney microstructures were developed to guide the free EpCAM-Ferrofluid that is present in clinical samples away from the imaging plane of the cells to reduce the distortive effect it has. (D) U-Groove microstructure that was developed to align the cells in channels.

$\mathrm{mm}$, the total length of the lines for each design is 2700,540 and $270 \mathrm{~mm}$. If we assume the average CTC to be at most $30 \mu \mathrm{m}$ in diameter, these designs can hold a maximum of $9 \times 10^{4}, 1.8 \times 10^{4}$ and $9 \times 10^{3}$ cells. The number of CTC that can be expected in a typical sample from a cancer patient after immunomagnetic enrichment from $7.5 \mathrm{ml}$ of blood ranges from 0 to $1 \times 10^{4}$.

The second microstructure, termed flat-top V-Groove, was designed to also align the cells in long lines, like the first structure. The difference with the first microstructure is that it features a flattened top of the V-Groove to allow for better, less distorted, imaging of the cells. Figure 4.1B shows the design of the second microstructure and the parameters that are involved. Parameter D indicates the spacing of the channels and L indicates the width of the flat area at the top of each channel. Several widths of the flattened area were designed to accommodate cells of varying size. Table 4.1 shows the combination of parameters that were used in the production of this 
TABLE 4.1 : Overview of parameter values that were used during production of the wafers. The parameters refer to those shown in Figure 4.1. For structure $\mathrm{C}$, three values are shown for parameter $\mathrm{D}$ and two for parameter L. This is to indicate that all combinations of parameters have been used in producing the respective wafer. The separated values for parameters $\mathrm{D}, \mathrm{L}$ and $\mathrm{H}$ for structure $\mathrm{D}$ indicate that only two combinations have been used.

\begin{tabular}{llll}
\hline Structure & Parameter & Values $[\mu \mathrm{m}]$ & \\
\hline V-Groove & $\mathrm{D}$ & $30,150,300$ & \\
\hline Flat-top V-Groove & $\mathrm{D}$ & 30 \\
& $\mathrm{~L}$ & $3,8,11,14,17$ & \\
\hline Chimney Groove & $\mathrm{D}$ & $30,150,300$ & \\
& $\mathrm{~L}$ & 2,3 & \\
\hline U-Groove & $\mathrm{H}$ & 15 & 480 \\
\hline & $\mathrm{D}$ & 150 & 80 \\
& $\mathrm{~L}$ & 50 & 30 \\
\hline
\end{tabular}

microstructure. A fixed spacing of $30 \mu \mathrm{m}$ was used and the width of the flat area was varied between 3 and $17 \mu \mathrm{m}$.

The third microstructure, also called chimney structure, was designed to minimize the influence of aggregates of unbound magnetic ferrofluid labels at the imaging surface. They are formed when these unbound labels in a sample are subjected to a magnetic field. These aggregates absorb and scatter fluorescence light, and transmitted light that is used for bright-field imaging. This causes a typical barcode like pattern in fluorescence images, making accurate signal quantification impossible. One way to minimize this effect is to collect the unbound magnetic labels in a different, higher, image plane as compared to the cells. This will cause the aggregates of magnetic labels to be out of focus when imaging the cells, thereby minimizing the effect they have on fluorescence quantification. The design of this microstructure is shown in Figure 4.1C. One additional parameter is introduced in this design, $\mathrm{H}$, which is the length of the 'chimney' channel that is located at the top of each V-Groove. A longer chimney will place the aggregates of magnetic labels further away from the cells, minimizing their influence. Therefore, $\mathrm{H}$ should be as large as possible within the limits of the technique that will be used to fabricate the structure. Table 4.1 indicates that only one value for parameter $\mathrm{H}$ was used. This is the maximum value that could be used in combination with the values ( 2 and $3 \mu \mathrm{m})$ that are used for the width of the chimney.

The fourth microstructure, termed U-Groove, features shallow channels at the top of each groove, to optimize the imaging of cells. Also, cells are now aligned in channels instead of lines, like in the first structure. 
Figure 4.1D shows the design of this microstructure. Two combinations of parameters were used in the design of this structure, as is shown in Table 4.1. The latter combination uses a significantly larger spacing than other structures, to minimize the effective area that needs to be imaged. However, this also limits the number of cells that can be accommodated inside the channels. For the structure featuring a spacing of $480 \mu \mathrm{m}$ this results in only 6 channels, each $80 \mu \mathrm{m}$ wide and an approximate maximum of $1 \times 10^{4}$ cells. The effective imaging surface area in this structure is $14.4 \mathrm{~mm}^{2}$ compared to the $81 \mathrm{~mm}^{2}$ of a glass imaging surface. This is a 5.6 fold reduction in available space for the cells.

\subsubsection{CLEANROOM TECHNOLOGIES FOR WAFER FABRICATION}

We have used three different anisotropic etching technologies to fabricate the microstructures on 4 inch silicon wafers. These techniques are explained below.

\section{Potassium Hydroxide ( $\mathrm{KOH}$ ) etching}

$\mathrm{KOH}$ etching is an anisotropic wet etching method that is used in the etching of silicon wafers. Etch rates depend on crystallographic orientation, doping of the silicon, concentration of $\mathrm{KOH}$ in water and the temperature of the $\mathrm{KOH}$ - Water bath in which the wafer is etched. The strong dependence of the etch rates on the orientation of the silicon crystal make this method highly anisotropic. This anisotropic property of $\mathrm{KOH}$ etching can be used to create trenches and V-shaped grooves. We will use it to create the V-shaped grooves that are typical for the microstructures.

Both silicon dioxide and silicon nitride can be used as masking materials for $\mathrm{KOH}$ etching. Silicon nitride is preferred when long etch times are involved because it etches more slowly than silicon dioxide. Relative etch rates as compared to silicon are in the order of 150:1 and >1650:1 for silicon dioxide and silicon nitride respectively [7].

$\mathrm{KOH}$ etching involves the placement of the wafer in a bath of $\mathrm{KOH}$ (at $\sim 30 \%$ dissolved in water) at a temperature of around $70^{\circ} \mathrm{C}$. The $\mathrm{KOH}$ dissolves in water and forms $\mathrm{K}^{+}$and $\mathrm{OH}^{-}$(hydroxide ions), the latter react with the silicon on the wafer according to the overall reaction [8]:

$$
\mathrm{Si}+2 \mathrm{OH}^{-}+2 \mathrm{H}_{2} \mathrm{O} \Leftrightarrow \mathrm{SiO}_{2}(\mathrm{OH})_{2}^{--}+2 \mathrm{H}_{2}
$$

This causes a silicon atom to be released from the surface, which is then carried away from the surface by diffusion. The removal of the silicon atom from a plane with a miller index of $\{111\}$ requires the breaking of most of the silicon - silicon bonds and is therefore the most unlikely to happen. This results in a low etch rate for these planes and a large anisotropy in etching. The relative etch rate [9] of the (110) orientation compared to the (111) orientation is around 250. This anisotropy also results in the 
characteristic angle of grooves that are etched using $\mathrm{KOH}$ etching. The angle of the groove with the wafer surface is determined by the two crystal planes involved, and is $54.74^{\circ}$. This is indicated by the complementary angle as shown in Figure 4.1A.

During the fabrication of the different wafers, $\mathrm{KOH}$ etching was used to etch the V-shaped grooves. This etch step was the last step that changed the profile of the microstructures significantly for each wafer. The $\mathrm{KOH}$ etching steps for each type of microstructure are shown in Appendices A.1, A.2 and A.3 respectively.

\section{Reactive ion etching}

Reactive ion etching $[10,11]$ is an anisotropic etching technique that uses a chemically active plasma to etch parts of the wafer. The wafer is contained in a low pressure reaction chamber on an electrode to which a radio frequency $(\mathrm{RF})$ field of $13.56 \mathrm{MHz}$ is applied. Introduced into the chamber are fluorinerich gasses like sulfur hexafluoride $\left(\mathrm{SF}_{6}\right)$ or carbon tetrafluoride $\left(\mathrm{CF}_{4}\right)$ along with an inert gas, usually Argon. A plasma builds up between the powered electrode (holding the wafer) and a grounded electrode. This plasma creates fluorine radicals from the fluorine-rich gasses in the chamber. These radicals are uncharged and therefore reach the silicon substrate with little or no directionality. When absorbed to the silicon surface, the fluorine radicals react with silicon atoms to form silicon fluorides $\left(\mathrm{SF}_{\mathrm{x}}, \mathrm{x}=1,2,3,4\right)$, which are volatile and readily leave the wafer surface. Since the fluorine radicals reach the surface with almost no directionality, this part of the reactive ion etching (RIE) process is isotropic.

Another aspect of RIE causes anisotropic etching and is explained next. Energetic electrons inside the plasma also collide with neutral gas (Argon) molecules, removing an electron from the gas molecule and leaving the molecule positively charged. The electrons in the plasma can follow the fast field changes that are induced by the RF field. This creates a negative DC bias voltage on the wafer immediately after formation of the plasma. The much heavier gas ions in the plasma are then accelerated towards the negatively charged wafer and they hit the surface in a perpendicular orientation. Impacting ions can either etch the silicon directly or assist in isotropic etching, by removing reaction products with low volatility. The latter aspect of ion impact enables further isotropic etching at the impacted site.

Directional etching by positively charged ions is less frequent than isotropic etching by fluorine radicals. However, due to the energetic impact of the ions, they strongly affect the local etching process and it is possible to obtain anisotropic etch profiles with reasonable aspect ratios after fine tuning of the process parameters.

The reactive ion etching method is used in the fabrication of the wafers containing the chimney- and the U-Groove microstructures. In the chimney 
structure, it is used to etch a silicon nitride $\left(\mathrm{Si}_{3} \mathrm{~N}_{4}\right)$ film as shown in Appendix A.2. In the U-Groove structure, it is used twice. First, to create an etch mask in silicon dioxide $\left(\mathrm{SiO}_{2}\right)$ in preparation for etching of the trenches. Second, to etch the silicon dioxide at the bottom of the trenches in preparation for $\mathrm{KOH}$ etching.

\section{Deep reactive ion etching}

Deep reactive ion etching (DRIE) is an etching technique that is commonly used in most cleanrooms. DRIE is used to create trenches and channels with near vertical sidewalls and large aspect ratios [12]. The DRIE method is an extension to the RIE process and also uses a combination of isotropic etching using fluorine radicals and anisotropic etching using gas ions. Since the isotropic etching by the fluorine radicals is spontaneous and the reaction products are highly volatile, they don't need ion activation to initiate or enhance their reaction or to remove reaction products from the wafer. To achieve the high aspect ratio anisotropic etching profiles that are typical of the DRIE process, sidewall passivation needs to be used. There are three main types of DRIE in use today: the black silicon method, cryogenic dry etching and the Bosch process. The latter one involves alternating cycles of etching and passivation and is the method we will use to fabricate vertical profiles in the microstructures.

The Bosch process uses a polytetrafluorethylene (PTFE) film to passivate the sidewalls during etching. The advantage of using such a film for passivation is that it is easily removed using low energy ion impact from the inert gas molecules and thus results in high mask selectivity's. For photoresist it is around 150:1 while for silicon dioxide $\left(\mathrm{SiO}_{2}\right)$ it can be much higher, up to 1000:1. Mask selectivity indicates the ratio with which the mask is etched compared to the material it masks.

The DRIE method is used in the fabrication of the wafers containing the chimney and U-Groove structures. It is used twice in the fabrication of the chimney structured wafer. First, to create narrow trenches of $2-3 \mu \mathrm{m}$ wide, that will eventually form the chimneys. Second, to etch the silicon around the silicon nitride $\left(\mathrm{Si}_{3} \mathrm{~N}_{4}\right)$ chimneys to let them stand out $\sim 15 \mu \mathrm{m}$. For the U-Groove microstructure, the DRIE method is used to create the channels that will be used to hold the cells.

\section{Teflon coating of wafers}

Wafers that have been processed in the cleanroom, using various etching technologies, are not yet suitable to be used for PDMS imprinting. This is due to the fact that PDMS adheres to the silicon dioxide layer, that inherently forms on bare silicon surfaces, during the curing process. This causes the PDMS to break up in small pieces when it is peeled away from the wafer. To prevent the PDMS from adhering to the etched wafer surface, 
an anti-sticking coating is applied to all wafers that are processed in the cleanroom. The anti-sticking coating that is applied is a perfluoronated octyltrichloro-silane (FOTS) [13] self assembled monolayer (SAM). This coating is commonly applied in micro-electromechanical systems (MEMS).

\subsubsection{PRODUCTION OF MICROSTRUCTURES ON SILICON WAFERS}

The four microstructure designs that were discussed previously were fabricated using the etching techniques that were explained in the previous section. The process steps involved in the production of the microstructures are described in detail in the Appendices A.1 - A.3. The V-Groove, flat-top V-Groove and chimney microstructures were produced by $\mathrm{C} 2 \mathrm{~V}$ (Enschede, The Netherlands). The U-Groove microstructure was produced by Lionix (Enschede, The Netherlands).

Scanning electron microscope (SEM) pictures of typical sections of each of the four developed wafer types are shown in Figure 4.2.

In Panel A several V-Grooves with a spacing of $300 \mu \mathrm{m}$ are shown. These start near the top of the image and run for $30 \mathrm{~mm}$ until the other end of the chip. Panel B shows the beginning of several flat-top V-Grooves with a spacing of $30 \mu \mathrm{m}$. Panel $\mathrm{C}$ shows part of several chimney structures with a spacing of $150 \mu \mathrm{m}$. The grid-like pattern at the bottom of the figure was included to prevent complete etching of the wafer outside the channels during the $\mathrm{KOH}$ etching. Finally, the U-Groove microstructure is shown in Panel D. The start of 5 channels can be seen near the bottom of the image. The point like shapes that are present between the start of two channels are due to the convex corner compensation scheme that was used [14].

A detailed view of the V-Groove structure that is shown in Figure 4.2A is shown in Figure 4.3.

It shows a SEM picture of an etch defect that occurred during $\mathrm{KOH}$ etching. This etch defect widens the top of the V-Groove locally, resulting in reduced alignment of the cells. The formation of these defects, which are termed hillocks $[15,16]$, can be explained using the $\mathrm{KOH}$ reaction equation:

$$
\mathrm{Si}+2 \mathrm{OH}^{-}+2 \mathrm{H}_{2} \mathrm{O} \Leftrightarrow \mathrm{SiO}_{2}(\mathrm{OH})_{2}^{--}+2 \mathrm{H}_{2}
$$

This reaction equation is bidirectional. Normal etching of silicon in the $\mathrm{KOH}$ process follows it from left to right. Silicon is removed from the surface and brought into solution by the help of hydroxide ions. However, when the local concentration of hydroxide ions is low due to a low $\mathrm{KOH}$ concentration or high temperature, the reaction can occur from right to left. This causes re-deposition of silicon onto the wafer surface. This re-deposition gives rise to random growth of pyramid structures, resulting in the etch defects as shown in Figure 4.3 . 

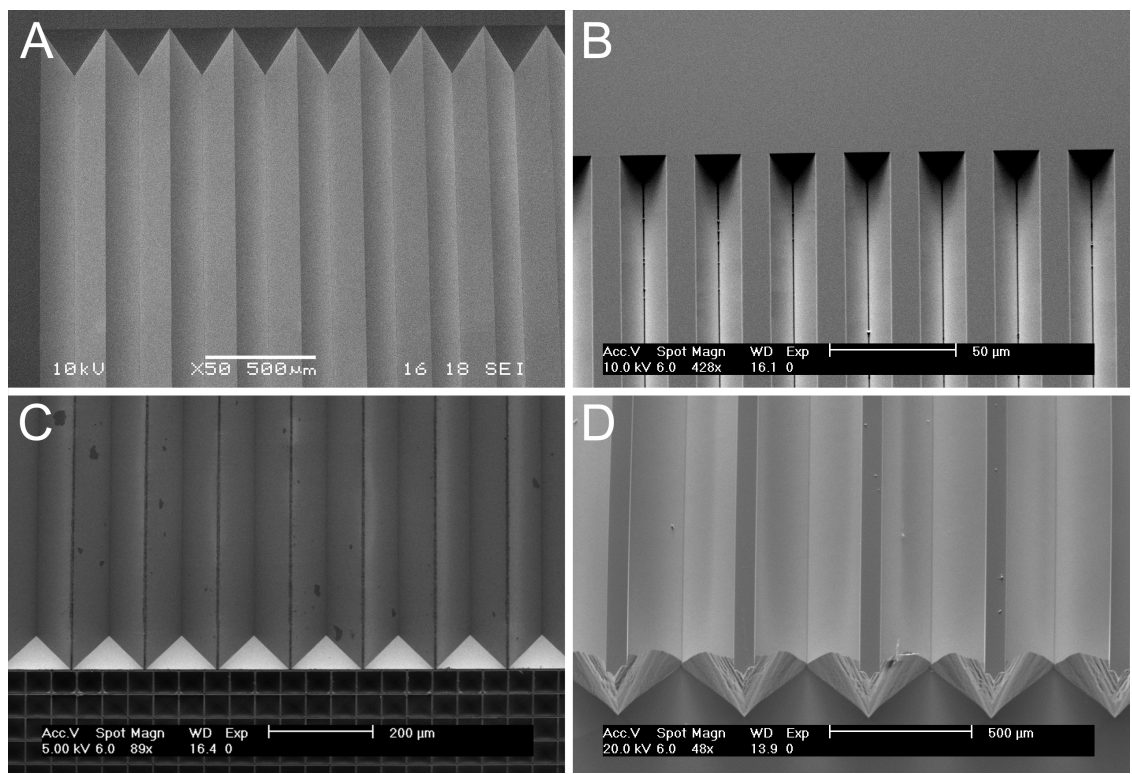

Figure 4.2 : Overview of SEM pictures of the completed wafers containing the same structures as described in Figure 4.1. All SEM pictures were taken normal to the wafer surface. (A) Top view of start of several V-Grooves with a spacing of $300 \mu \mathrm{m}$. This SEM picture shows clearly defined V-Grooves with sharp edges. However, there were some manufacturing defects in parts of the wafer that caused local flattening of the tip of the V-Groove. See Figure 4.3. (B) Top view of part of the etched silicon wafer containing the flat-top V-Groove microstructure. The SEM picture shows the start of several etched V-Grooves with a spacing of $30 \mu \mathrm{m}$ and a flat area with a width of $11 \mu \mathrm{m}$. (C) Top view of chimney microstructure with a spacing of $150 \mu \mathrm{m}$ and a chimney height of $15 \mu \mathrm{m}$. Again, the start of a few grooves is shown. The multiple square features at the bottom of the image serve to prevent complete etching of the wafer outside of the grooves during $\mathrm{KOH}$ anisotropic etching. (D) SEM picture showing the start of 5 U-Groove channels with a spacing of $480 \mu \mathrm{m}$; channels are $80 \mu \mathrm{m}$ wide and $30 \mu \mathrm{m}$ high. The pencil shaped ends of the channels were formed as a result of a technique to compensate for convex corners.

\subsubsection{PDMS IMPRINTING}

\section{Imprint material}

We chose PDMS as the imprinting material because it is easy to manufacture multiple replicates from 1 master wafer. It replicates even the finest details and the transmission spectrum is well suited for excitation of fluorophores over the wavelength range that is used for fluorescent imaging $(350-800 \mathrm{~nm})$. We compared the transmission spectra of three commonly used imaging surface materials using a spectrophotometer (Shimadzu, Japan), the results are shown in Figure 4.4. Also, the cured PDMS is flexible and can therefore easily be removed from the wafer. 


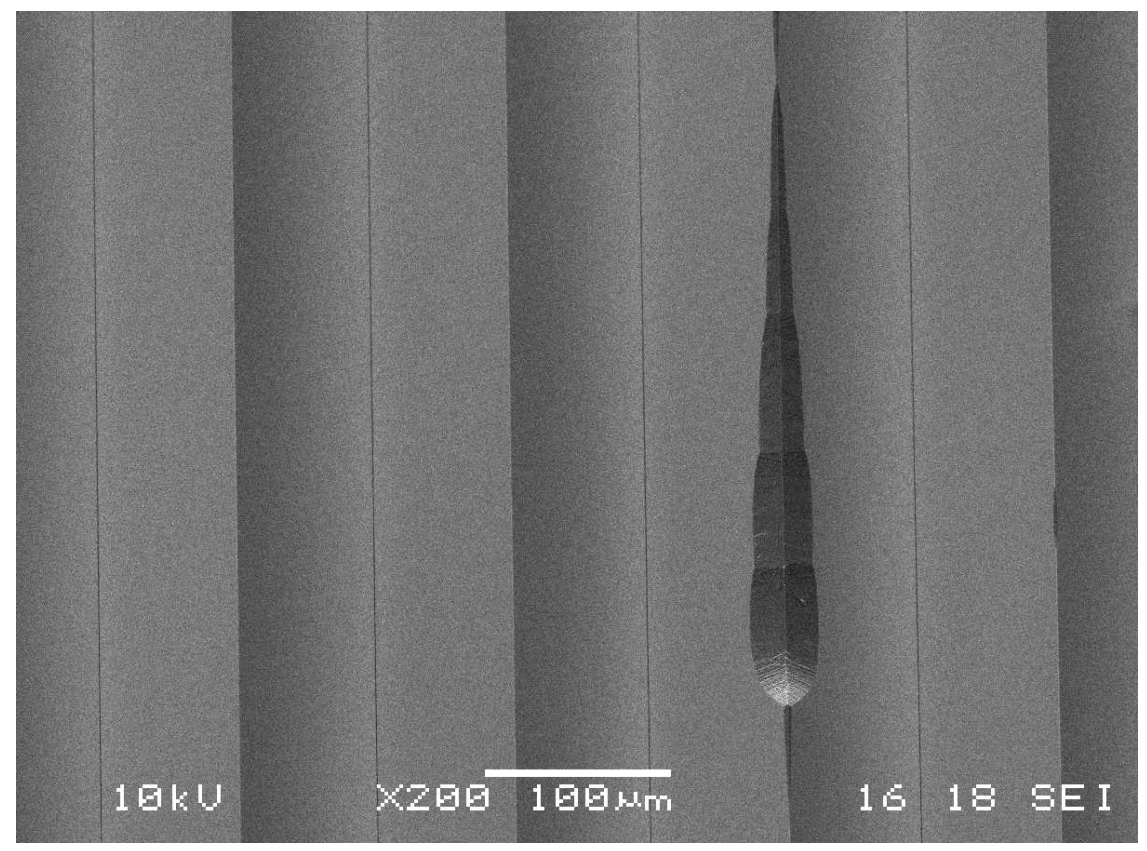

FIGURE 4.3 : SEM picture showing an etch defect that was formed during anisotropic $(\mathrm{KOH})$ etching of the silicon wafer to produce the V-Groove structures. These defects, termed hillocks, are not related to mask defects, surface conditions of the silicon or lithography problems $[7,8]$. They are intrinsic to the anisotropic etching process and are frequent at low $\mathrm{KOH}$ concentrations during anisotropic etching.

\section{Imprinting technique}

PDMS imprints were made using the Sylgard 184 elastomer kit (Dow Corning, Midland, MI, USA). The kit consists of two components: a base (component A) and a curing agent (component B) that were combined in a ratio of 10:1 (A:B, by weight). After thorough mixing of the two components, the mixture is placed into a vacuum system. Pressure inside the system is reduced to approximately $1 \mathrm{mbar}$ and this is maintained for about 2 minutes. The low pressure causes the trapped air bubbles in the mixture, that were created during mixing, to rise to the top. After 2 minutes, the pressure is increased to normal atmospheric pressure over a few seconds, causing most of the air bubbles to burst. To remove all of the air bubbles in the PDMS mixture, this procedure is repeated 3 times followed by a prolonged period of low pressure of around 15 minutes. This generally leaves a completely bubble-free PDMS mixture that is ready to pour.

To create a PDMS imprint that is evenly thick across its surface, a custom build device is used. This device was specifically designed to hold a 


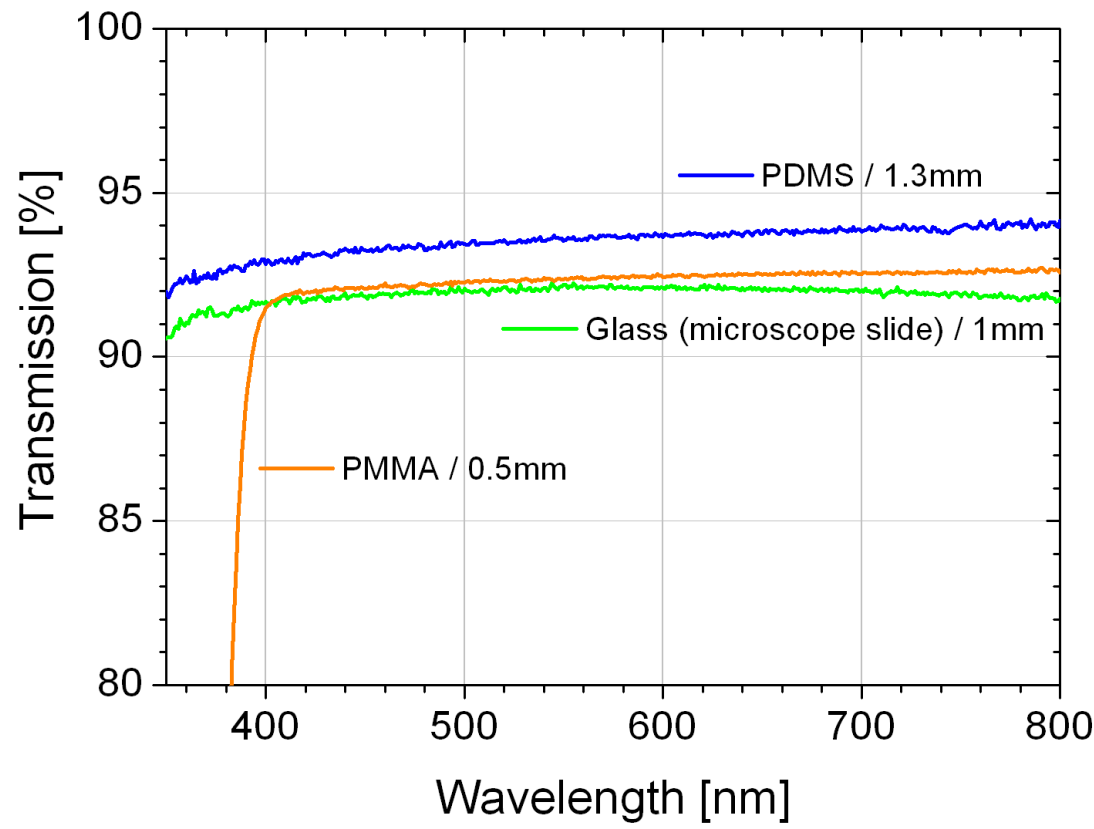

FIGURE 4.4 : Comparison of three commonly used imaging surface materials. The glass and PDMS have a transmission of over $90 \%$ between 350 and $800 \mathrm{~nm}$. The transmission of PMMA drops sharply for wavelengths lower than $400 \mathrm{~nm}$ and is therefore not suited for excitation using a UV laser or the $365 \mathrm{~nm}$ line of a mercury arc lamp. The values in the legend indicate the thickness of the material that was analyzed in the spectrophotometer (Shimadzu, Japan) to determine the transmission spectrum.

standard 4 inch wafer and comes with several spacers of various thicknesses that can be placed on top of the wafer, determining the thickness of the cured PDMS. These spacers then hold a cover plate that squeezes out all excess PDMS. The whole system is clamped together and placed into an oven at $90^{\circ} \mathrm{C}$. After 2 hours, it is removed from the oven and left to cool to room temperature. Then the clamping system and cover plate are removed, after which the PDMS is carefully removed from the wafer and placed into a separate container.

Figure 4.5 shows part of a PDMS imprint featuring the U-Groove microstructure that was cut from a standard chip. All four images were made using a scanning electron microscope ((SEM), FEI, Netherlands). The first image (A) shows an overview of several channels and the edge of the chip, in the background, which is at the same height as the tip of the grooves. Also clear are the near-perfect flatness of the sloped sidewalls and the channels themselves. The second through fourth images (B-D) show the PDMS imprint in increasingly greater detail. The smoothness of 

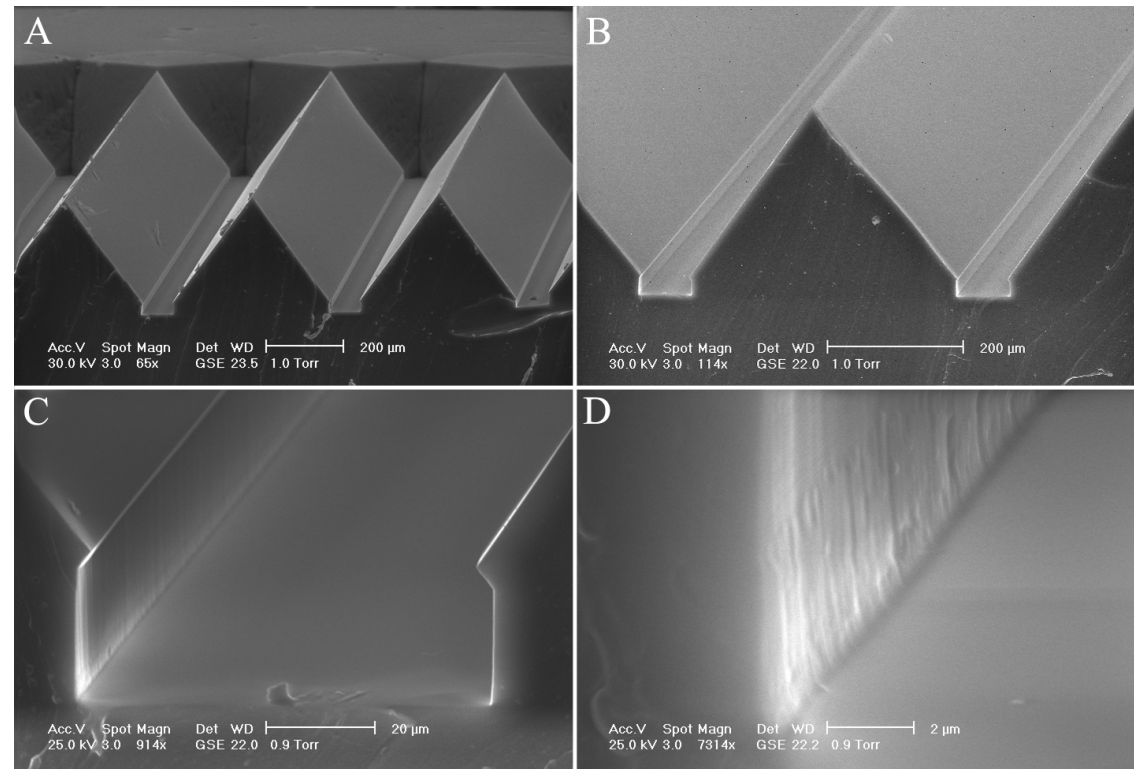

FIGURE 4.5 : Overview of 4 SEM pictures of PDMS imprint of foxhole microstructure. (A) A piece of approximately $4 \mathrm{~mm}$ in length was cut from a standard chip. The edges of several channels are clearly visible as well as the near-perfect flatness of the sloped sidewalls and the channels themselves. (B) A second piece with a thickness of approximately $3 \mathrm{~mm}$ was cut from the center of a PDMS chip and 2 channels are visible. (C) Close-up view of left channel from (B). The small underetch that was present in the wafer is replicated in the PDMS structure. Again, the smoothness of the channel's lower surface (imaging surface) is clearly visible. (D) Highly magnified view of the lower-left edge of the start of the channel.

the channel's lower surface (imaging surface) is clearly visible in the third image.

\subsubsection{PREPARATION OF CELL ANALYSIS CARTRIDGES USING PDMS CHIPS}

The cell analysis cartridges that are used in the CellTracks system have been developed by Veridex. They are designed to fit tightly in a magnetic yoke assembly (Magnest Cell Presentation Device) that consists of two wedge-shaped magnetic poles. The cell analysis cartridge consists of a cuvet $\left(30 \times 2.7 \times 4 \mathrm{~mm}^{3}(\mathrm{~L} \times \mathrm{W} \times \mathrm{H})\right.$, volume $\left.=324 \mu \mathrm{l}\right)$ and additional plastic material to position the cuvet correctly in the magnetic field and hold the plug that is used to seal the cartridge. Standard cell analysis cartridges consist of a cuvet that is sealed at the top with a piece of borosilicate glass (CellSearch, Veridex).

To use the microstructures to align and concentrate the cells, the cuvet is sealed with a piece of PDMS that has the same dimensions as the glass chip. These PDMS chips are cut manually from the PDMS imprint. They 


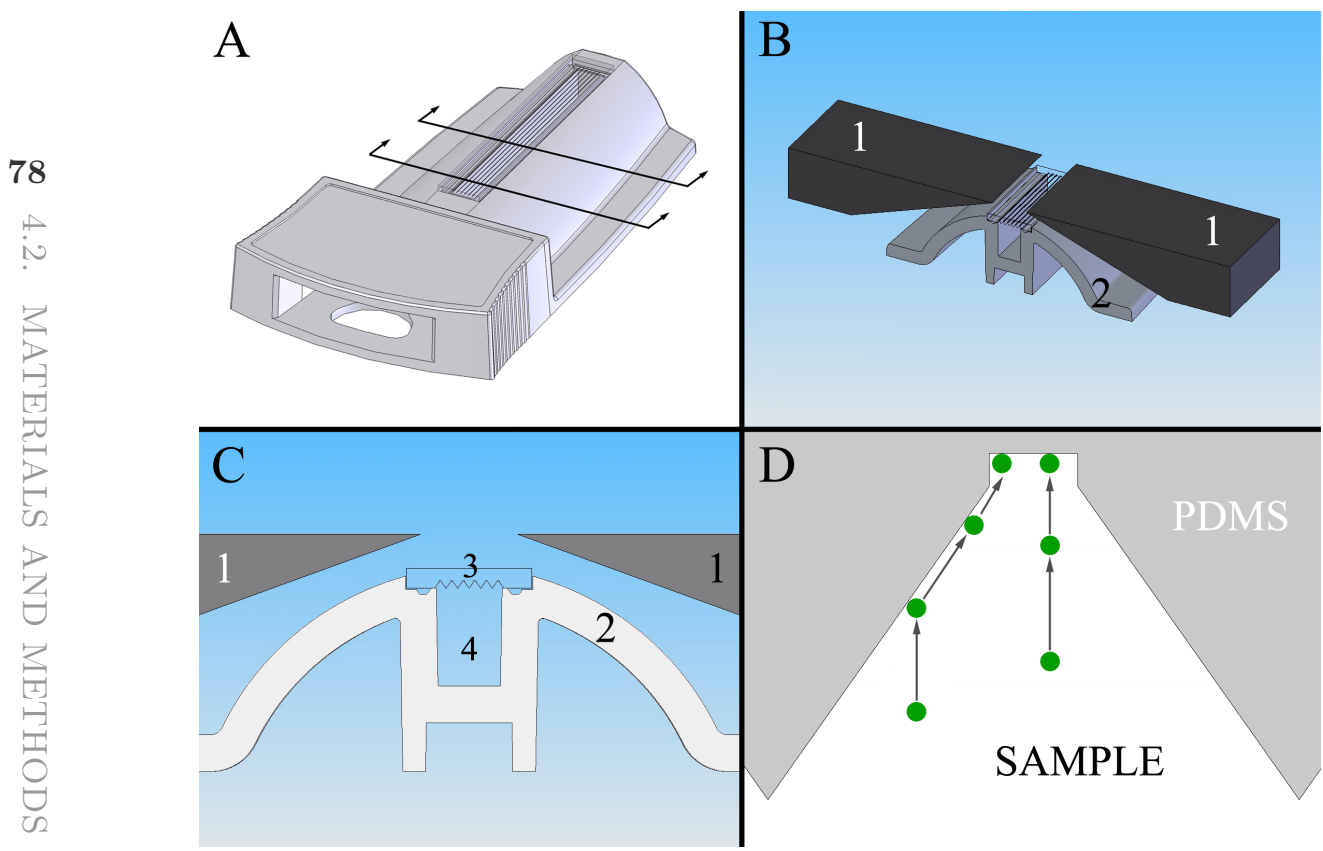

FIGURE 4.6 : Overview of cartridge, positioning in magnest and cell movement in microstructures. (A) 3D view of CellSearch cartridge with U-Groove microstructured top. The two lines with arrows on the end indicate the location of the cut-through view that is shown in B. (B) Cut-through view showing positioning of cartridge underneath the magnetic poles. Indicated are: 1: Magnetic poles, 2: Cartridge. (C) Front view of magnetic poles, cartridge and PDMS top. Indications 1 and 2 are equal to those in B, 3: PDMS top, 4: chamber formed by cartridge and PDMS top. (D) Detail view of cell movement inside a groove. Green circles represent the movement, indicated by arrows, of two cells going to the imaging surface.

are positioned on top of the cuvet and alignment and positioning is checked under a microscope. When the PDMS chip is correctly positioned on top of the cuvet, it is glued in place using UV-curable glue (Norland, Cranbury, NJ, USA). The glue is applied around the outside edges of the PDMS chip and cured using an X-Cite 120 XL illumination system (EXFO, Mississauga, Ontario, Canada). This completes the cell analysis cartridge and it can now be used for measurements.

Figure 4.6 shows an overview of positioning of a cartridge (A) under the magnetic poles $(\mathrm{B}, \mathrm{C})$ and the movement of cells inside the microstructures (D). The cartridge and PDMS top form the chamber that holds the sample as shown in (C), indicated by 2, 3 and 4 . Cell movement inside a groove is illustrated by two cells, indicated by green filled circles, moving to the analysis surface. The left cell is guided by the PDMS as indicated by the arrows. The right cell moves straight up to the imaging surface. For 
structures with a large spacing compared to the channel width, most cells will move to the imaging surface similar to the left cell as indicated in the figure.

\subsubsection{CANCER CELL LINE SAMPLES}

All experiments featuring cancer cell line cells contain cells from the cancer cell line SKBR-3 (ATCC, Middlesex, UK). The cells were cultured in RPMI 1640 Glutamax-I medium (Invitrogen, Carlsbad, CA, USA). Cells were harvested and subsequently fixed using paraformaldehyde (PFA) at a concentration of $1 \%$ for at least 30 minutes. Then, the cells were washed twice using Phosphate Buffered Saline (PBS) after centrifugation at 300 $\mathrm{g}$ for 5 minutes. The PFA fixes the SKBR-3 cells by cross-linking free amino groups within the cell. No significant effects of the cross-linking are expected on the behavior of the cells in relation to the PDMS structures.

The tumor cell line sample was magnetically labeled by incubating $1 \mathrm{ml}$ of fixed SKBR-3 cell suspension $\left(\sim 1.1 \times 10^{3} / \mathrm{ml}\right)$ with $200 \mu \mathrm{l}$ anti-EpCAMFerrofluid $(0.2 \mathrm{mg} / \mathrm{ml})$. These ferrofluids consist of coated paramagnetic particles $\left(\mathrm{Fe}_{2} \mathrm{O}_{3}\right)$ labeled with anti-EpCAM antigens and have a diameter of 150 to $200 \mathrm{~nm}$. To label the nucleus and cytokeratins of the SKBR-3 cells, $5 \mu \mathrm{l}$ Hoechst $33342(10 \mathrm{mg} / \mathrm{ml})$ and $50 \mu \mathrm{l}$ Cytokeratin-PE were added respectively. The sample was then incubated in a quadrupole magnet [17] for 5 minutes and then vortexed. This procedure was repeated 3 times, employing enhanced surface loading of the cells [18]. After incubation, the sample was washed to remove unbound EpCAM-Ferrofluid. To this end, the sample was diluted to $7 \mathrm{ml}$ using PBS and centrifuged at $300 \mathrm{~g}$ for 5 minutes, after which the supernatant was aspirated. This washing step was repeated twice. The resulting sample contained around 50 SKBR-3 cells/ $\mu$ l, had no visible unbound EpCAM-Ferrofluid present and could now be used to assess the image quality, capture efficiency and alignment efficiency in the different microstructures.

Note that patient samples that are processed in the AutoPrep system are not washed extensively to remove unbound Ferrofluids.

\subsection{Results}

\subsubsection{PDMS IMPRINTING OF MICROSTRUCTURES}

The chimney microstructure, as shown in Figure 4.1C, was successfully fabricated in the clean room. However, during the first PDMS imprinting it became apparent that the chimney parts of the wafer were tapered negatively. This means that the width of the channel that should be used to collect unbound ferro fluid, was larger at the top than at the bottom. Therefore it was almost impossible to remove the PDMS from the wafer because it stuck in between the chimney structures. The PDMS layer could only be 
peeled away from the wafer by using considerable force which caused the chimney channels on top of the V-Groove to break off from the wafer. This rendered the wafer unsuitable for further PDMS imprinting. As a result, no experiments were performed with the chimney microstructures. The three other microstructures were successfully fabricated and yielded no problems during PDMS imprinting.

\subsubsection{IMAGE QUALITY OF CELLS IMAGED IN THE MICROSTRUCTURES}

An important property of cell aligning microstructures is that they should present the cells at an analysis surface in a condition which allows unobstructed and distortion free imaging, both when emitted fluorescence is detected or when the cells are imaged using bright-field illumination. When using the original Nickel lines, parts of the cells remain invisible because they are obstructed by the Nickel lines. However, those parts of the cells that are not obstructed, can be imaged without distortion, because the cells are located underneath a piece of glass with constant thickness.

All three tested microstructures are made of the optically transparent material PDMS, giving an unobstructed view of the cells. Some distortion of the fluorescence or bright-field signal coming from the cells in the microstructures is expected because the refractive index of PDMS is 1.41 and that of the sample containing the cells is typically around 1.35. This difference in refractive index causes some distortion, specifically at sharp transitions in the structures as in the top of the V-Groove structure shown in Figure 4.1A.

To compare the image quality between the three fabricated microstructures, we used a sample that contains cells of the tumor cell line SKBR-3. Cartridges with the different analysis surfaces were scanned using the CellTracks TDI system. The entire analysis surface of each cartridge was scanned for 3 colors (DAPI, PE and bright-field) at a speed of $1 \mathrm{~mm} / \mathrm{sec}$. A neutral density filter (Optical density $=1.0$ ) was used to prevent saturation of the fluorescence images. Raw images were corrected for the slightly inhomogeneous profile of the square laser spot and the background was subtracted.

Figure 4.7 shows typical images of SKBR-3 cells aligned in the three microstructures. The images show an overlay of the bright-field image (gray values), fluorescence image for DAPI (nucleus, blue) and fluorescence image for PE (Cytokeratin, green). Panel A in Figure 4.7 shows the alignment of several single SKBR-3 cells and a cluster of SKBR-3 cells in the V-Groove microstructure with a spacing of $300 \mu \mathrm{m}$. Only a small section of 1 of the $\mathrm{V}$-Grooves is shown in this image for clarity. The thin horizontal line that can be seen in the bright-field image of the $\mathrm{V}$-Groove corresponds to the tip of the V-Groove being in focus. The cluster of cells is aligned at a small angle with respect to the tip of the V-Groove, which is common for 

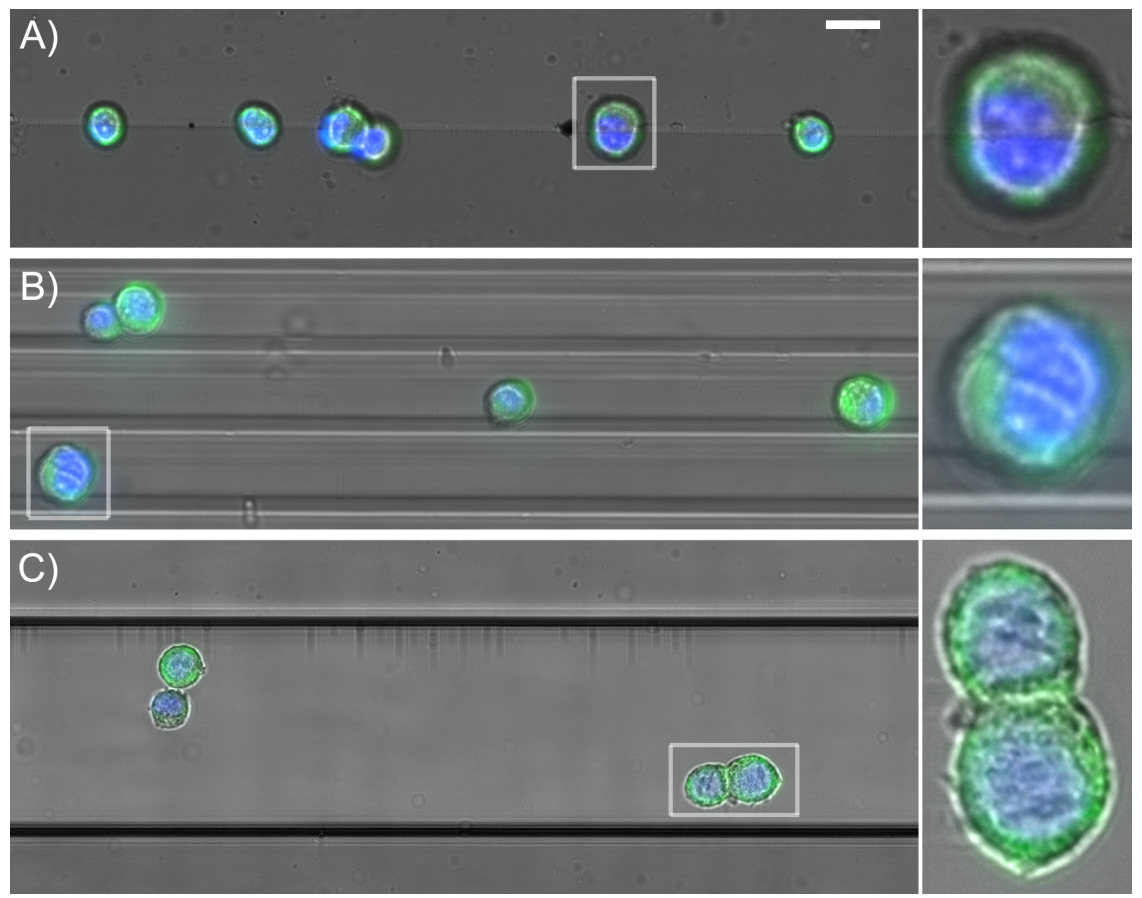

Figure 4.7 : Overview of cell alignment in three of the four microstructures that were developed using SKBR-3 cells. The images show an overlay of the bright-field image (gray values), fluorescence image for DAPI (nucleus, blue) and fluorescence image for PE (Cytokeratin, green). The left part of each section shows several cells aligned in the respective structures and the right side gives a detailed view of one of the cells. (A) Alignment of 4 single SKBR-3 cells and a cluster of 2 cells in the V-Groove microstructure. Only a small section of 1 of the V-Grooves is shown in this image for clarity. The cluster is aligned at a small angle, which is common for cell clusters. However, single cells are usually located in the center of the V-Groove and therefore correctly aligned. (B) Alignment of 3 single SKBR-3 cells and 1 cluster of 2 cells in the flat-top V-Groove microstructure. Again, the cluster of 2 cells is aligned under a small angle. The flat-top part of this microstructure is $14 \mu \mathrm{m}$ wide. (C) Alignment of 2 single SKBR-3 cells and 1 cluster of 2 cells in the U-Groove microstructure that features, in total, 6 channels that are $80 \mu \mathrm{m}$ wide. The small vertical stripes that can be seen in the bright-field image are aggregated ferrofluid particles that were still in the sample. Scale bar $=20 \mu \mathrm{m}$ (only for the left side of the image). 
cell clusters. However, single cells are usually located in the center of the V-Groove and therefore correctly aligned.

Panel B in Figure 4.7 shows the alignment of several single cells and a cell cluster in the flat-top V-Groove microstructure. The flat-top part of this microstructure is $14 \mu \mathrm{m}$ wide and the average diameter of SKBR-3 cells is around 15-20 $\mu \mathrm{m}$. This allows for an unobstructed view of most of the cell if it would align in the center of the $30 \mu \mathrm{m}$ wide channels. However, the majority of single SKBR-3 cells are aligned with one edge of the cell touching the sloped sidewall of the V-Groove. This causes a similar distortion in fluorescence and bright-field images as shown in Figure 4.8. Image quality is better than the $\mathrm{V}$-Groove structure but not as good as can be obtained with a flat glass imaging surface.

Panel C in Figure 4.7 shows 2 single SKBR-3 cells and 1 cluster of cells in the U-Groove microstructure that features, in total, six channels that are each $80 \mu \mathrm{m}$ wide.

Only a small part of one of the channels is shown, the edges are clearly visible by the dark horizontal lines in the bright-field image. This microstructure has an entirely flat and level imaging surface at the top of each channel. This allows for a better image quality of the cells, which is comparable to a flat glass imaging surface.

A more detailed view of one of the cells in each type of structure is given to the right of each of the panels in Figure 4.7. These show that the image quality of cells in the U-Groove microstructure is significantly better than for cells that are aligned in the other two structures.

Figure 4.8 shows the effect of the tip of the V-Groove microstructure on imaging of SKBR-3 cells that are located underneath. Each row in the figure is divided into 3 columns. The first column shows the DAPI fluorescence image, the second column shows the Cytokeratin-PE fluorescence image and the last column shows the bright-field image. The images that are shown in the first row (A) were made with the focus $5 \mu \mathrm{m}$ above the tip of the $\mathrm{V}$-Groove. Images in the next row (B) were made with the focus on the tip of the V-Groove and the last row of images corresponds to the focus being $5 \mu \mathrm{m}$ below the tip. The last row corresponds to the best focus position for the cell, showing the most detail in images in that row.

\subsubsection{CAPTURE EFFICIENCY OF CELLS ON THE VIEWING SURFACE OF THE MICROSTRUCTURES}

The purpose of the microstructures is to align magnetically labeled cells at the imaging surface of a cartridge. In theory the magnetic field, induced by the magnetic poles of the Magnest, will force all cells to move towards the imaging surface. However, in reality, cells may get stuck on their way to the top of the cartridge. One possible cause is local roughness of the PDMS surface. Also, debris present in the sample may adhere to the PDMS and then may hold cells back. Aggregates of two or more cells may get stuck on 

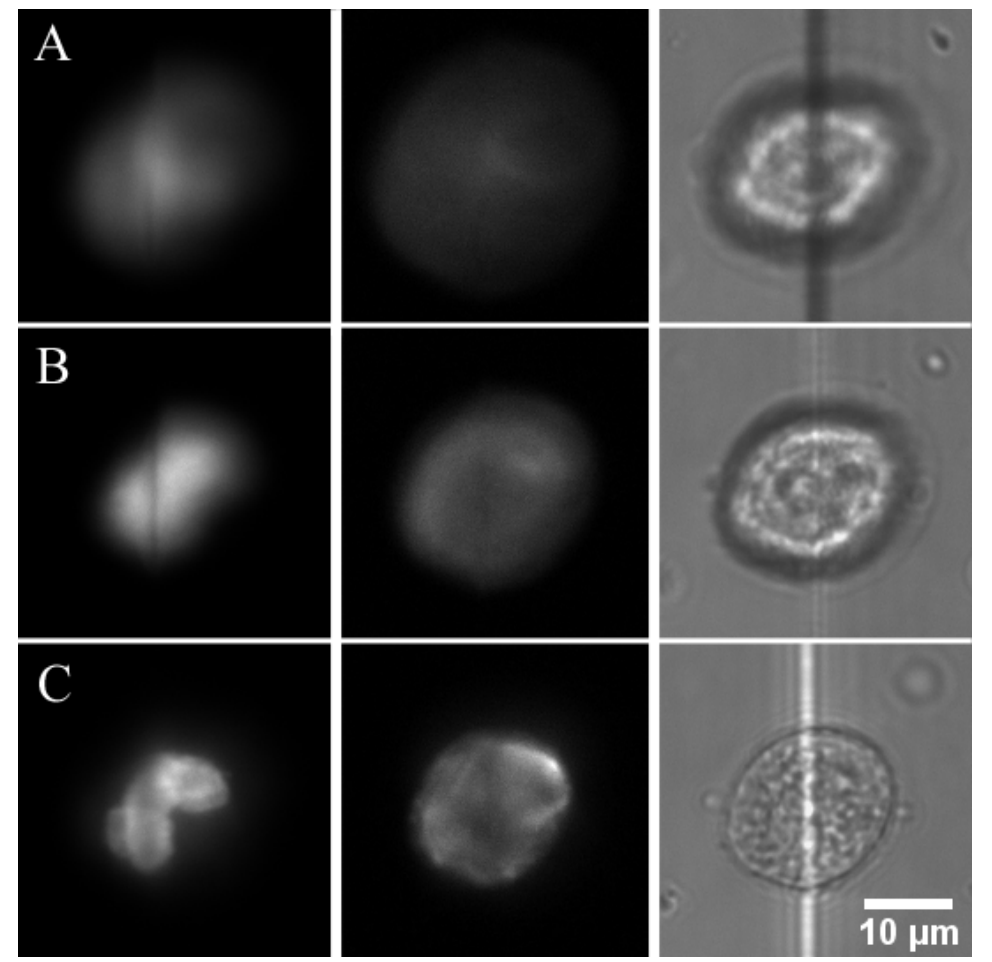

FIGURE 4.8 : Effect of the V-Groove structure on imaging of a SKBR-3 cell. First column: DAPI fluorescence images showing the cell nucleus, second column: Phycoerythrin fluorescence showing cytokeratins and third column: bright-field image showing cell morphology and structure. Rows: focus $5 \mu \mathrm{m}$ above (A), on (B) and $5 \mu \mathrm{m}$ below (C) the tip of the V-Groove.

the lower tip of a groove. At very high concentrations the imaging surface may get fully covered by cells and further cells simply cannot reach the imaging surface and are trapped.

The capture efficiency of the different microstructures was determined using samples containing approximately 1000 SKBR-3 cells. The determination was repeated five times for each different microstructure. All cartridges were scanned using the CellTracks TDI system. In each measurement the data was processed using custom built software written in ImageJ [19]. In this routine, first, all scanned images are corrected for uneven illumination and the background is subtracted. Then, a gate is set on the DAPI fluorescence channel using a fixed threshold and parameters of interest are determined. Next all events are analyzed and SKBR-3 cells are identified as $\mathrm{DAPI}^{+} \mathrm{PE}^{+}$events that have a round to oval morphology. The number of incorrectly positioned cells was determined separately by observation in a fluorescence microscope (Nikon Eclipse E400, Nikon, Melville, NY, USA). 


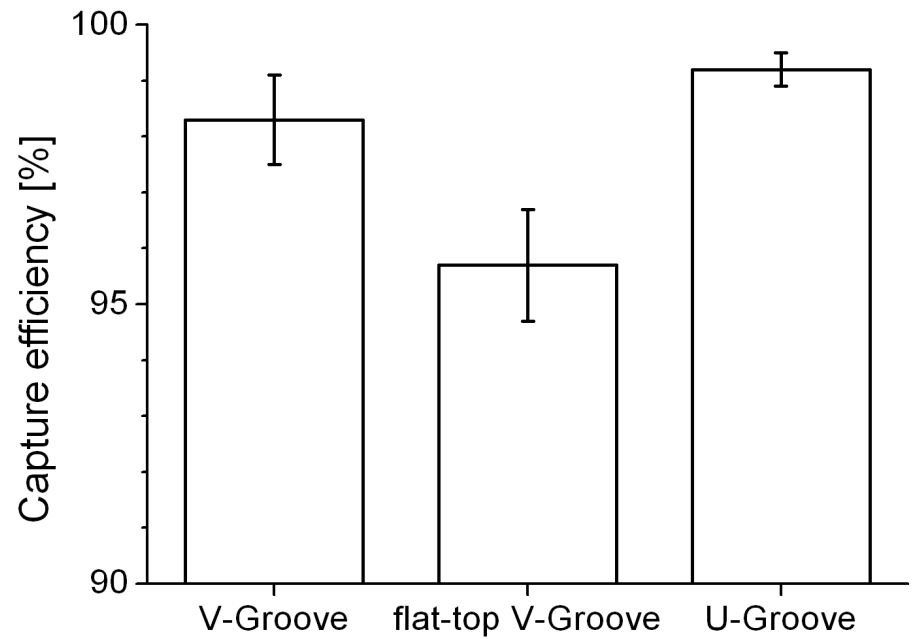

Figure 4.9 : Capture efficiency of the three tested types of microstructures. The capture efficiency for the three structures is $98.3,95.7$ and $99.2 \%$ respectively with standard deviations of $0.8,1.0$ and $0.3 \%$.

The capture efficiency is next calculated as the ratio between the number of correctly positioned cells and the total number of cells in the sample. The total number was determined by adding the amount of incorrectly positioned cells to the number of correctly positioned cells.

Figure 4.9 shows the capture efficiency of SKBR-3 cells using the 3 tested microstructures. The error bars represent the standard deviation of the five repetitions. As is clear from the figure, the flat-top V-Groove structure has the lowest capture efficiency. This is mainly due to the low spacing of this structure of only $30 \mu \mathrm{m}$. As a result, it has the most sloped side walls of the tested structures. Cells mostly get stuck near the bottom tip of a V-Groove, were the sloped sidewalls start.

The standard deviation of the capture efficiency for the V-Groove and the flat-top $\mathrm{V}$-Groove structure is larger than it is for the U-Groove structure. For the V-groove, this is mainly due to etch defects as shown in Figure 4.3. They locally broaden the position that a cell can end up in. As the number of defects varies over the surface of the etched wafer, it may not be equal for each cartridge. For the flat top structure, the larger deviation is mainly due to the difficulty of correctly assessing whether a cell is correctly placed or not. 
TABLE 4.2 : Overview of total imaging surface area in different cell aligning structures

\begin{tabular}{llllll}
\hline Structure & $\begin{array}{l}\text { Spacing } \\
{[\mu \mathrm{m}]}\end{array}$ & $\begin{array}{l}\text { Width } \\
{[\mu \mathrm{m}]}\end{array}$ & $\begin{array}{l}\text { Length } \\
{[\mathrm{mm}]}\end{array}$ & $\begin{array}{l}\text { Surface } \\
\text { area }\left[\mathrm{mm}^{2}\right]\end{array}$ & $\begin{array}{l}\text { Fraction of avail- } \\
\text { able surface }\end{array}$ \\
\hline V-Groove & 30 & 12 & 30 & 32.4 & 0.40 \\
& 150 & 12 & 30 & 6.5 & 0.08 \\
& 300 & 12 & 30 & 3.2 & 0.04 \\
\hline Flat-top V-Groove & 30 & 12 & 30 & 32.4 & 0.40 \\
& 30 & 14 & 30 & 37.8 & 0.47 \\
& 30 & 17 & 30 & 45.9 & 0.57 \\
\hline U-Groove & 150 & 50 & 30 & 27.0 & 0.33 \\
& 480 & 80 & 30 & 14.4 & 0.18 \\
\hline
\end{tabular}

\subsubsection{ALIGNMENT EFFICIENCY OF THE MICROSTRUCTURES}

The total surface area that is available for depositing the cells is $81 \mathrm{~mm}^{2}$ $(2.7 \times 30 \mathrm{~mm})$, the surface of the sample cartridge. It is important to assess the ability of the microstructures to concentrate the cells onto a small area of the total surface. The minimum area on which the cells can be concentrated is dependent on the parameters shown in Table 4.1. A larger spacing will result in a smaller imaging surface area, and therefore a higher concentration of cells on that imaging area. Wider channels will yield a larger imaging area. The smallest 'channel' width is reached in the V-Groove structure, which can align the cells along lines with a width that is only determined by the cell diameter. The smallest number of channels is present on the U-Groove microstructure, which has only six channels. The effective imaging area of each microstructure is given in Table 4.2. The average diameter of a cell is assumed to be $12 \mu \mathrm{m}$. Structures that have channels narrower than $12 \mu \mathrm{m}$, are assumed to be as wide as a cell diameter.

To locate each cell at the surface, we usually specify two parameters, one along the long side of the cartridge and one along the short side. To indicate the distribution of cells on the different surfaces, we use one parameter only; its position measured along the short side. We call this the position of the cell 'across the cartridge'.

Figure 4.10 shows the distribution of cells across the cartridge for the three tested microstructures and for a glass imaging surface. In all four cases the binsize is taken to be $10 \mu \mathrm{m}$.

In case of the standard glass imaging surface, more cells appear to be located at the edge of the cartridge than in the middle. This is due to the low amount of free Ferrofluid in the sample in combination with the type of Magnest that was used. In a sample with more free Ferrofluid, the excess particles form a homogeneous layer across the imaging surface which prevents the cells from moving towards the edges of the cartridge. The 

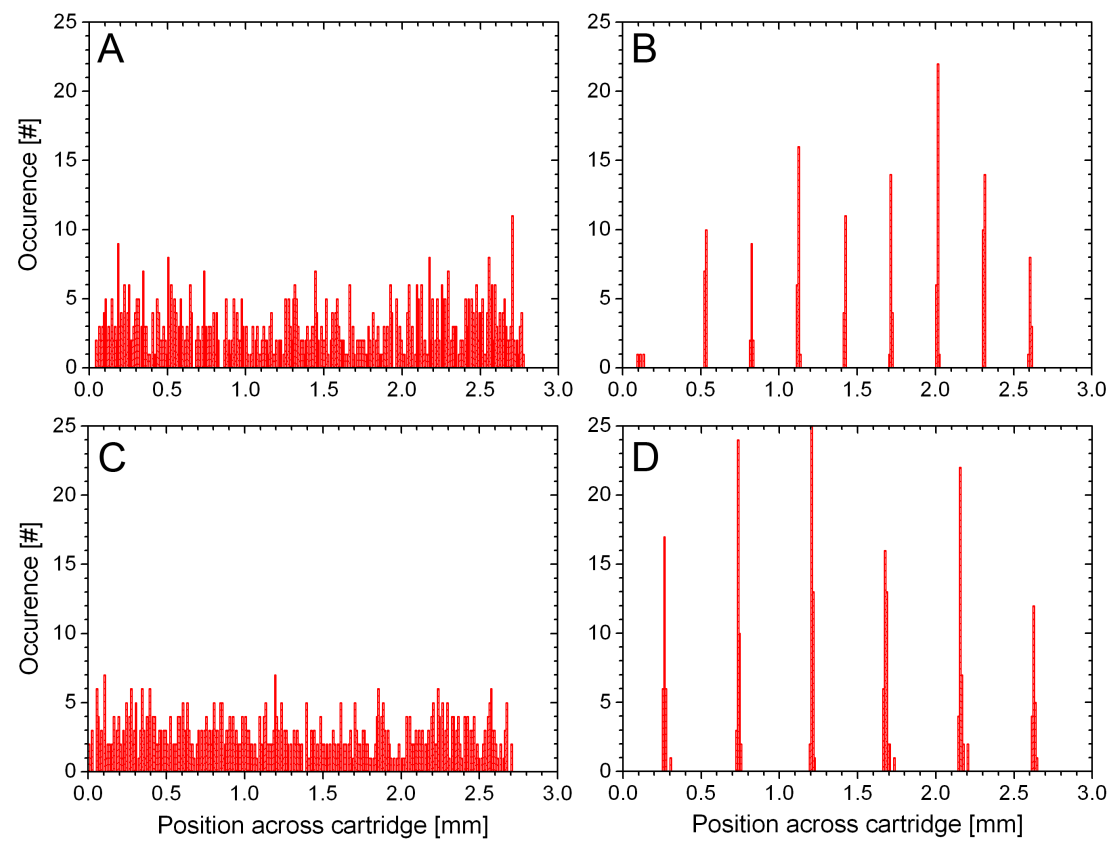

Figure 4.10 : Overview of alignment efficiency of the three tested microstructures and a glass surface. Histograms show the relative location of SKBR-3 cells within the cartridge. Bin size is $10 \mu \mathrm{m}$. (A) Glass analysis surface, (B) V-Groove structure, (C) flat-top V-Groove structure and (D) U-Groove structure.

microstructures in turn, prevent excess movement of cells to the outside of the cartridge due to physical restriction of the cells inside the channels.

As is evident from the figures, the high alignment efficiencies of the V-Groove- and U-Groove structure, in combination with their excellent capture efficiencies, make these structures most suitable for application in a laser scanning system, reducing the time needed for scanning substantially.

\subsubsection{FLUORESCENCE DETECTION EFFICIENCY OF BEADS IN THE MICROSTRUCTURES}

The fluorescence detection efficiency for cells in a microstructure will depend on the material of which the microstructure is made and the shape the particular structure has. The mean fluorescence intensity (MFI) and the variation of that intensity $(\mathrm{CV})$ were determined using beads. The results are shown in Figure 4.11. The $\mathrm{CV}$ for the beads in the cartridge with the glass analysis surface and that of the U-Groove microstructure are similar. However, the $\mathrm{CV}$ for the beads in the $\mathrm{V}$-Groove structures is significantly worse compared to the other two surfaces. This is most likely due to the 

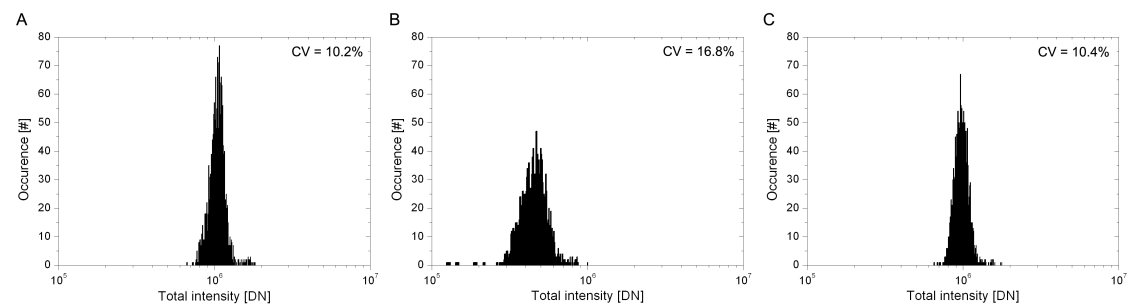

Figure 4.11 : Fluorescence detection efficiency of the tested microstructures. The fluorescence intensity of EnvyGreen beads, located at the analysis surface of a CellSearch cartridge is shown for different collecting microstructures. Fluorescence was excited with the $491 \mathrm{~nm}$ laser and emitted light was collected by the emission filter for PE. (A) glass surface. (B) surface with V-Grooves with a spacing of $150 \mu \mathrm{m}$, (C) surface with U-Grooves with a spacing of $80 \mu \mathrm{m}$. In all three cases 2500 beads were measured.

distortive effect that the V-shaped groove has on the imaging, as discussed in the section on image quality.

\subsection{Discussion}

In image cytometry usually a solution of cells is applied to a surface that is then scanned optically to obtain images of the cells at the surface. This imaging method is most effective when the density of target cells at the analysis surface is high. This limits the actual area that has to be scanned and therefore the time required to get statistically relevant data. The above assumes that it is adequate to only image part of a sample, by e.g. imaging a fixed number of cells to reach statistically acceptable numbers. One example is the observation, on a microscope slide, of a white blood cell sample containing large subpopulations like granulocytes and lymphocytes. This method is also comparable to the method used in most flow cytometry measurements.

However, in case of CTC analysis using the CellSearch system, the entire analysis surface of the CellTracks cartridge has to be imaged to avoid not detecting all relevant events. This is particularly important in the analysis of these rare cells as, after magnetic separation of the CTC's, only 1 to 10 CTC may be present amidst many blood cells and debris. Further magnetic separation does not yield an improvement in CTC enrichment and one has to turn to different ways to reduce the analysis time.

In this paper we presented and tested a novel method to concentrate magnetically labeled CTC from a low concentration solution onto a small area. To do so, we prepared grooved PDMS structures that are imprints of molds made by clean room technologies. Their surfaces are smooth, leading to the majority of cells being forced by the external magnetic field of the Magnest to the correct location in the lines or channels formed in the grooved structure. This is illustrated by the high capture efficiency of 
96-99\%, which is excellent. In general, the quality of the cell images and the fluorescence collection efficiency was satisfactory. Some disturbance of the images occurred for the V-Groove and flat-top V-Groove structures that deposit the cells in very narrow lines. The U-Groove structure has the best image quality, which is comparable to imaging a cell through a piece of Pyrex glass as is used in the current CellTracks cartridge. Differently shaped structures reduced the imaging surface by factors varying between 1.75 and 25 , leading to a potential reduction of $96 \%$ in scan time. In case of the U-Groove structure, the sample is concentrated into 6 channels that are $80 \mu \mathrm{m}$ in width, leading to a total reduction of imaging surface area by a factor of 5.6. The expected reduction in analysis time for a CTC sample scanned for 4 colors using the CellTracks TDI system is a factor of $\sim 3$, reducing the total analysis time from 36 to 13 minutes.

We conclude that our method for reducing the area to be scanned works well and indeed deposits cells on a fraction of the original surface area, with a small loss in target cell number. It is evident that the method works best if the concentration of non-target cells and debris is low. Its application is not limited to the analysis of CTC, in principle any sample with magnetically labeled cells with a low final concentration can be used. Also the excess concentration of magnetic label particles, in our case ferrofluid particles of $150 \mathrm{~nm}$, should be kept as small as possible. We also developed a technique to remove most of the excess ferrofluid particles from the sample, which is discussed in Chapter 7.

To develop the structures, we used PDMS micromolding, a technique that is often used in micro-electrical mechanical systems (MEMS) [20-23]. The main advantages of using PDMS are its excellent optical properties in the near UV and visible wavelength ranges $(350-800 \mathrm{~nm})$, its flexibility and the possibility to produce several imprints from the same wafer in a short period of time. The last advantage was especially important during the development phase of these structures. Several designs could therefore easily be tested. Improvements in the fabrication of the structures, to optimize the end product, may be obtained by using a different molding material, for instance PMMA [24]. Another option to be able to use a more rigid material for the microstructures would be to fabricate the 'positive structures' in the silicon wafer. Then make an imprint containing the 'negatives' in PDMS and use it as a mold to fabricate the positive structures in e.g. PMMA or polyurethane.

The method presented here is closely related to the orientation of cells by gravitation in $\mathrm{V}$ grooves [1]. This method used gravity to align and concentrate cells into V-Grooves were they were then scanned using a laser spot. In literature we did not find any different method that similarly reduces the area to be scanned. 


\subsection{REFERENCES}

[1] B. de Grooth, T. Geerken, and J. Greve, "The cytodisk: A cytometer based upon a new principle of cell alignment," Cytometry, vol. 6(3), pp. 226-233, 1985.

[2] A. Tibbe, B. de Grooth, J. Greve, P. Liberti, G. Dolan, and L. Terstappen, "Optical tracking and detection of immunomagnetically selected and aligned cells," Nature Biotechnology, vol. 17, pp. 1210-1213, 1999.

[3] A. Tibbe, B. de Grooth, J. Greve, G. Dolan, C. Rao, and L. Terstappen, "Magnetic field design for selecting and aligning immunomagnetic labeled cells," Cytometry, vol. 47(3), pp. 163-172, 2002.

[4] A. Tibbe, B. de Grooth, J. Greve, P. Liberti, G. Dolan, and L. Terstappen, "Cell analysis system based on immunomagnetic cell selection and alignment followed by immunofluorescent analysis using compact disk technologies," Cytometry, vol. 43(1), pp. 31-37, 2001.

[5] A. Tibbe, B. de Grooth, J. Greve, C. Rao, G. Dolan, and L. Terstappen, "Cell analysis system based on compact disk technology," Cytometry, vol. 47(3), pp. 173182, 2002.

[6] A. Tibbe, B. de Grooth, J. Greve, G. Dolan, and L. Terstappen, "Imaging technique implemented in celltracks system," Cytometry, vol. 47(4), pp. 248-255, 2002.

[7] K. Williams, K. Gupta, and M. Wasilik, "Etch rates for micromachining processing Part II," Journal of Microelectromechanical Systems, vol. 12, pp. 761-778, 2003.

[8] H. Seidel, L. Csepregi, A. Heuberger, and H. Baumgaertel, "Anisotropic etching of crystalline silicon in alkaline solutions. i. orientation dependence and behavior of passivation layers," Journal of the Electrochemical Society, vol. 137, pp. 3612-3626, 1990.

[9] K. Sato, M. Shikida, Y. Matsushima, T. Yamashiro, K. Asaumi, Y. Iriye, and M. Yamamoto, "Characterization of orientation-dependent etching properties of single-crystal silicon: Effects of $\mathrm{KOH}$ concentration," Sensors and Actuators and A: Physical, vol. 64, pp. 87-93, 1998.

[10] F. Laermer, Comprehensive Microsystems, pp. 217-233. Elsevier, 2008.

[11] H. Jansen, H. Gardeniers, M. de Boer, M. Elwenspoek, and J. Fluitman, "A survey on the reactive ion etching of silicon in microtechnology," Journal of Micromechanics and Microengineering, vol. 6, pp. 14-28, 1996.

[12] F. Marty, L. Rousseau, B. Saadany, B. Mercier, O. Français, Y. Mita, and T. Bourouina, "Advanced etching of silicon based on deep reactive ion etching for silicon high aspect ratio microstructures and three-dimensional micro- and nanostructures," Microelectronics Journal, vol. 36, pp. 673-677, 2005.

[13] M. Geerken, T. Van Zanten, R. Lammertink, Z. Borneman, W. Nijdam, C. Van Rijn, and M. Wesseling, "Chemical and thermal stability of alkylsilane based coatings for membrane emulsification," Advanced Engineering Materials, vol. 6, pp. 749-754, 2004.

[14] M. Bao, C. Burrer, J. Esteve, J. Bausells, and S. Marco, "Etching front control of $<100>$ strips for corner compensation," Sensors and Actuators and A: Physical, vol. 37-38, pp. 727-732, 1993.

[15] H. Han, R. Boudreau, T. Bowen, S. Tan, and M. Reed, "Etching defects on koh etched silicon - implementation of silicon bench technology for low cost packaging," in Proceedings - Electronic Components and Technology Conference, IEEE, 1994. 
[16] S. Tan, M. Reed, H. Han, and R. Boudreau, "Mechanisms of etch hillock formation," Journal of Microelectromechanical Systems, vol. 5, pp. 66-72, 1996.

[17] P. Liberti, Y. Wang, W. Tang, B. Feeley, and D. Gohel, "Apparatus and methods for magnetic separation featuring external magnetic means," USA Patent 5,466,574, 1995.

[18] P. Liberti, C. Rao, and L. Terstappen, "Optimization of ferrofluids and protocols for the enrichment of breast tumor cells in blood," Journal of Magnetism and Magnetic Materials, vol. 225(1-2), pp. 301-307, 2001.

[19] W. Rasband, "Imagej," http://rsbweb.nih.gov/ij/index.html, vol. -, pp. -, 1997 2010 .

[20] S. Sia and G. Whitesides, "Microfluidic devices fabricated in poly(dimethylsiloxane) for biological studies," Electrophoresis, vol. 24, pp. 3563-3576, 2003.

[21] J. McDonald and G. Whitesides, "Poly(dimethylsiloxane) as a material for fabricating microfluidic devices," Accounts of Chemical Research, vol. 35, pp. 491-499, 2002.

[22] D. Duffy, J. McDonald, O. Schueller, and G. Whitesides, "Rapid prototyping of microfluidic systems in poly(dimethylsiloxane)," Analytical Chemistry, vol. 70, pp. 4974-4984, 1998.

[23] J. McDonald, D. Duffy, J. Anderson, D. Chiu, H. Wu, O. Schueller, and G. Whitesides, "Fabrication of microfluidic systems in poly(dimethylsiloxane)," Electrophoresis, vol. 21, pp. 27-40, 2000.

[24] R. Mukhopadhyay, "When pdms isn't the best," Analytical Chemistry, vol. 79, pp. 3248-3253, 2007. 


\section{CHAPTER}

\section{IMPLEMENTATION OF CELL ALIGNMENT STRUCTURES IN Celltracks TDI}

In this chapter the implementation of the U-Groove microstructure from Chapter 4 into the CellTracks TDI system is presented.

Cell alignment microstructures were made in PDMS and glued onto cell analysis cartridges. CellSearch cartridges, with a plain Pyrex glass imaging surface, were used for comparison. Cultured cells from the tumor cell line SKBR-3 were used as a model for real CTC from cancer patients. The CellTracks TDI system, an image cytometer that continuously acquires images while scanning the sample, was used to scan the cells in both types of cartridges.

A bright-field autofocus method was developed that works up to a concentration of unbound ferrofluid of $2 \mu \mathrm{g} / \mathrm{ml}$. Interpolation of focus positions is done using a 2D spline fit for each channel separately, improving the accuracy of the feed-forward focusing algorithm. Imaging times for a CellSearch sample chamber were reduced from 38 to $14 \mathrm{~min}$, and the image analysis time for the resulting data from 65 to $27 \mathrm{~min}$. Also, the linearity of recovery is good, with a correlation coefficient of 0.996 and a slope of 0.937 in the clinically relevant range of $0-1000 \mathrm{CTC} / 7.5 \mathrm{ml}$.

The new microstructured analysis cartridge reduces the effective imaging area by concentrating the cells mechanically. The CellTracks TDI system was successfully adapted, resulting in a scan time for a typical sample of 12 minutes. 


\subsection{INTRODUCTION}

The number of Circulating Tumor Cells (CTC) in the blood of a cancer patient correlates to the prognosis [1-6]. In a clinical setting, it is essential to enumerate CTC after i.e. initiation of a new therapy, as they may illustrate the effectiveness of this new therapy. We use the CellSearch system to immunomagnetically enrich CTC from whole blood. The enriched sample is then analyzed in a CellSearch cartridge. In Chapter 4 we discussed microstructures that were developed to optimize imaging of the cells at the analysis surface of a cartridge. The most effective structure, the U-Groove microstructure, is now used to concentrate CTC from a low concentration sample in six channels along the imaging surface.

This chapter will discuss the implementation of that microstructure into the CellTracks TDI system. The scanning system is adapted with respect to the autofocus, feed forward focusing algorithm and reduced surface area of the microstructured cartridge. Also, the linearity of recovery between CTC imaged in a CellSearch- and microstructured cartridge is determined. Furthermore, the effects of unbound ferrofluids on image quality will be evaluated.

\subsection{Materials And Methods}

\subsubsection{SAMPLE PREPARATION}

Cells from the cancer cell line SKBR-3 (ATCC, Middlesex, UK) were used for all experiments. The cells were cultured in RPMI 1640 Glutamax-I medium (Invitrogen, Carlsbad, CA, USA). Cells were harvested and subsequently fixed using paraformaldehyde (PFA) at a concentration of $1 \%$ for at least 30 minutes. Then, the cells were washed twice using Phosphate Buffered Saline (PBS) after centrifugation at $300 \mathrm{~g}$ for 5 minutes.

Most tumor cell line samples are prepared by first labeling the SKBR-3 cells immunomagnetically using anti-EpCAM-Ferrofluid. The Epithelial Cell Adhesion Molecule (EpCAM) is used to select epithelial cells, which are normally not found in the blood. The enhanced surface loading technique [7] was used to maximize the labeling of available EpCAM sites on the cells. To accomplish this, the sample is introduced in a quadrupole magnet [8]. This sweeps the ferrofluid particles to the outside of the tube past the cells. Once all the ferrofluid particles are at the outer wall of the tube, usually after 3-5 minutes, the sample is vortexed and this process is repeated three times. After immunomagnetic labeling, the cells are fluorescently labeled using DAPI to stain the nucleus and Cytokeratin-PE (CK-PE) to stain the Cytokeratin structure inside the cells.

After the labeling steps, the sample is washed, if necessary for the experiment, to remove unbound EpCAM-Ferrofluid and excess fluorescent dyes. To this end, the sample was diluted to $7 \mathrm{ml}$ using PBS and centrifuged 
at $300 \mathrm{~g}$ for 5 minutes, after which the supernatant was aspirated. This washing step was repeated twice.

\subsubsection{CELL PRESENTATION CARTRIDGES}

Microstructured cartridges that can be used in conjunction with the Magnest Cell Presentation Device were fabricated by glueing PDMS imprints onto cartridges without a top surface. A small amount of Norland optical adhesive UV curable glue nr. 68 (Norland, Cranbury, NJ, USA) was applied along the outer edges of the PDMS chip and cured using an EXFO X-Cite 120 fluorescence illumination system (Exfo, Ontario, Canada). The PDMS imprints feature six channels that are $80 \mu \mathrm{m}$ wide and $30 \mathrm{~mm}$ long, as discussed in Chapter 4 . The outer dimensions of a PDMS chip are $31.5 \times 5$ $\mathrm{mm}$, with the six channels laying inside the chip. This results in the concentrated and aligned cells being in a plane about $300 \mu \mathrm{m}$ above their normal height inside the Magnest assembly. However, a requirement for successful scanning of any surface inside the CellTracks TDI system is that it is located within the $400 \mu \mathrm{m}$ travel range of the objective positioning system. The system is aligned in such a way that cells in a cartridge with a glass top are usually in focus in the middle of that range.

Without any changes, this would mean that the channels would be out of range of the objective positioning system. To put the cells at the same relative height inside the Magnest assembly, the aluminum holder underneath the magnetic poles inside a Magnest was lowered by $300 \mu \mathrm{m}$.

Cartridges featuring a glass top were obtained from a CellSearch CTC Kit (Veridex, Raritan, NJ, USA).

\subsubsection{CELLTRACKS TDI SYSTEM}

The CellTracks TDI system $[9,10]$ is an improved version of the original CellTracks setup as developed by Tibbe et. al. [11, 12]. A detailed description of the setup is given in Chapter 2.

\section{Hardware}

In short, the CellTracks TDI system is a laser scanning image cytometer. It uses a TDI camera to acquire images of the cells while they are scanned at a constant velocity. Fluorescent labels are excited using UV, green and red lasers, and a high power LED underneath the sample is used to create bright-field images. All three laser lines are combined into an overlapping bundle and focused into an approximately square profile, of $180 \times 180 \mu^{2}$, using a beam homogenizing system. A $40 \times / 0.6 \mathrm{NA}$ objective is used with a final magnification of 32.25 . 


\section{Scanning}

To analyze a sample, it is placed inside a cell analysis cartridge, which is then inserted into the Cell Presentation Device. This assembly is then placed into a holding system which is mounted on a stack of two linear translation stages. The combination of these two stages allow for a sample travel of $50 \times 50 \mathrm{~mm}^{2}$.

To scan the sample, first the focus position at 9 locations on the imaging surface is determined. Then, a second order polynomial function is fitted to the 9 focus positions to determine the intermediate focus points that will be used during actual scanning of the cells. Since a second order two dimensional polynomial function is used for fitting, the minimal number of focus points that needs to be determined is 9 , three in each direction of the imaging surface. A script, written in Labview, is used to automate this feed forward focus algorithm.

To cover the entire imaging area of a glass cartridge, 16 scans, each 180 $\mu \mathrm{m}$ wide, are made. This is repeated for each additional color that needs to be imaged. At a default imaging speed of $1 \mathrm{~mm} / \mathrm{sec}$, each single color scan of the entire surface takes approximately 9 minutes, including overhead time.

\section{Image analysis}

Raw images that are obtained by the CellTracks TDI system are processed and analyzed using the image analysis program ImageJ [13]. The background of all fluorescence images is first removed using the rolling ball method. Then each fluorescence image is corrected for inhomogeneous illumination by multiplying each pixel column with a predefined correction factor [9]. Next, images are stitched together in groups of four to reduce the number of events that are on the border of an image.

The next step in image analysis is to detect the events of interest. For a CTC sample, events of interest are defined to be CK-PE positive. A fixed threshold is used on the PE fluorescence image. Each event that is detected is extracted from the original image, for all colors, and stored in a separate multipage TIFF file that combines all detected events. Extraction of events is done using a fixed window of $30 \times 30 \mu \mathrm{m}^{2}$.

After all events have been extracted from all original images, quantitative fluorescence and morphological parameters are calculated based on an automatic Otsu threshold [14, 15]. Typical parameters include total fluorescence intensity, circularity, size and ratios between different colors.

Finally, the events are reviewed using a custom built Labview program. This program displays the quantitative information of the events in dotplots and histograms and also displays the fluorescence and bright-field images for each selected event. An operator then uses a fixed set of rules to determine which events are CTC and which are, for example, leucocytes or debris. 


\subsection{Results}

\subsubsection{SCANNING OF MICROSTRUCTURED CARTRIDGES}

To correctly scan the imaging surface of the microstructure cartridge in the CellTracks TDI system, changes have to be made to the software and scanning procedures. First, a new autofocus method was developed that can automatically determine the focus position inside one of the six channels based on bright-field imaging. This autofocus method is then used to determine the focus position on a grid of $6 \times 6$ points. These points are then interpolated to determine intermediate focus values.

\subsubsection{AUTOFOCUS ON DIFFERENT POSITIONS}

To be able to automatically determine the focus position inside any of the six channels, a bright-field autofocus method was developed. The autofocus method that is used for a standard cartridge involves the measurement of the reflection of the red laser profile off of the glass - sample interface. A 'combined' image is made in this autofocus method that integrates the reflection of the laser spot over the entire range of the microscope objective positioning system, which is $400 \mu \mathrm{m}$, while the TDI camera integrates the reflected signal in an elongated image. A fixed line rate is used to trigger the TDI camera since the stages are not moving and therefore not generating an encoder signal. The focus position is determined by finding the part of the combined image that has the highest contrast as compared from the center to the edge of the image. This corresponds to the square homogenized laser spot being in focus [9]. For this method to work, the entire laser spot needs to be reflected. The microstructures are only $80 \mu \mathrm{m}$ wide, while the laser spot including boundary is around $240 \mu \mathrm{m}$ wide. This makes it impossible for the entire laser spot to be reflected and thus the current autofocus method cannot be used for the microstructures.

Therefore, a new autofocus method was developed. In this case, the bright-field image of the channel is integrated over the entire range of the objective positioning system, while the sample is at rest. This results in a characteristic image as shown in Figure 5.1A.

The two vertical lines that transition from white to black against the gray background are the borders of the channel. The location inside the image where these lines are the thinnest corresponds to the focus position. To find that part of the image, the variation in gray levels within a small window is determined. This window is scanned over the entire image to determine the variation in gray levels along the image. Then, the derivative is taken from the variations across all the sections, as shown in Figure 5.1B. The sharp peak, in this example at $170 \mu \mathrm{m}$, is then detected using the peak detect function in Labview. A window of 5 pixels is used and the peak that has the greatest maximum value is selected. 

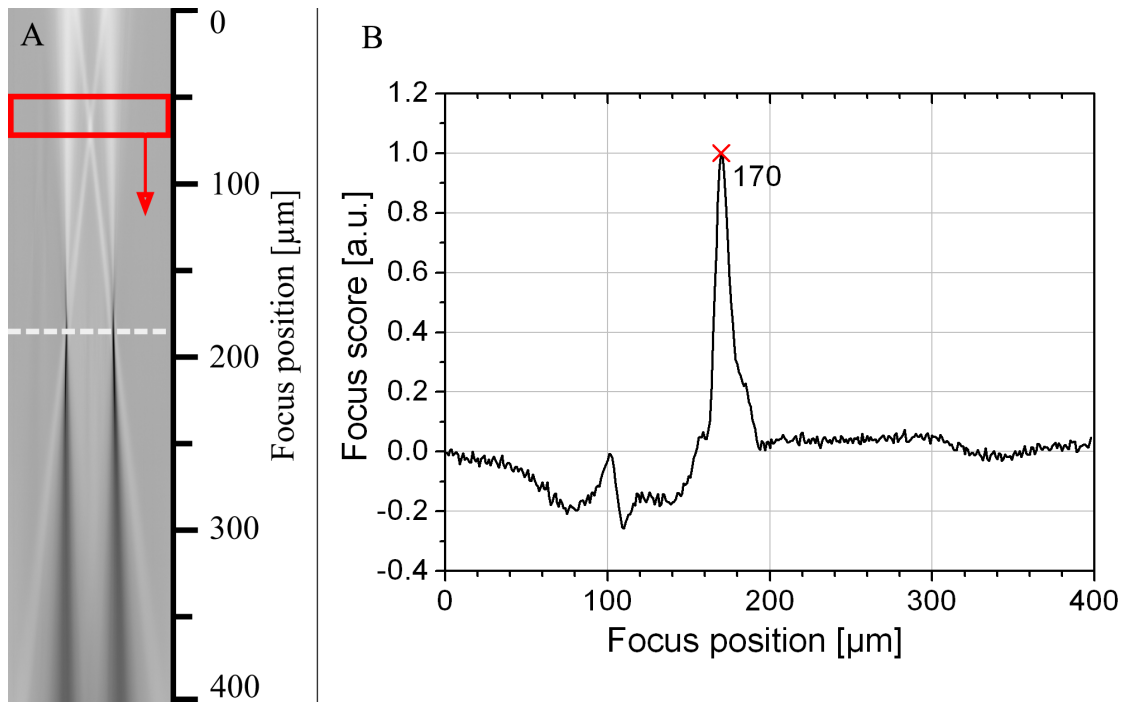

Figure 5.1 : Bright-field autofocus method implemented in the CellTracks TDI system. The objective is moved through its entire travel range of $400 \mu \mathrm{m}$ while the TDI camera integrates the bright-field signal. (A) The integrated image is then analyzed using a scanning window with a height of 50 pixels (depicted in red). The variation in gray levels inside the window is determined and then the window is shifted down and the process is repeated until the end of the image. (B) The derivative of the variation in gray levels as determined from (A). The sharp peak at $170 \mu \mathrm{m}$ corresponds to the transition from white to black in the image and is indicated by the white dashed line in (A)

The performance of this autofocus method was determined by comparing it to manually determined focus positions along a channel. The focus position of each of the six channels was manually determined at ten locations. Then the developed autofocus method was used to determine the focus position at the same locations. The average difference between the automatic and manual focus determination method is $11.1 \mu \mathrm{m}$, with a standard deviation of $1.5 \mu \mathrm{m}$.

Another aspect of the performance of the autofocus method is related to the concentration of unbound ferrofluid inside the sample. These antiEpCAM labeled $\mathrm{Fe}_{2} \mathrm{O}_{3}$ ferrofluid particles are around $150 \mathrm{~nm}$ in diameter. When their concentration at the imaging surface is too high, the ferrofluid forms aggregates that will block and scatter significant amounts of the bright-field light. This will reduce the effective contrast of the transitions in the U-Grooves in the autofocus image as shown in Figure 5.1A. To test the effect of unbound ferrofluid on the autofocus method, samples with unbound ferrofluid concentrations ranging from 0 to $200 \mu \mathrm{g} / \mathrm{ml}$ were tested. Results indicated that the autofocus method works reliably up to a concentration of unbound ferrofluid of around $2 \mu \mathrm{g} / \mathrm{ml}$. 


\subsubsection{INTERPOLATION OF FOCUS POSITIONS}

The second change to the CellTracks TDI system that is necessary concerns the interpolation method that is used to generate intermediate focus points for use during scanning of the entire imaging area. Currently, a two dimensional polynomial interpolation method is used. This method is well suited for use in combination with a glass imaging surface. The borosilicate glass on a CellSearch cartridge is relatively rigid. Apart from a small tilt in the positioning of the glass surface, the largest deviation from a perfectly flat surface is usually a slight bending of the glass in the middle [9].

Since the PDMS is more flexible than the standard glass top, it can be expected that there are more higher order variations in the focus positions across the entire imaging surface. Also, the PDMS microstructure has only 6 channels and each neighboring channel is $480 \mu \mathrm{m}$ away, making it unlikely that a $2 \mathrm{D}$ fit will get the focus right at these 6 locations.

To test whether a second order 2D fit will be suitable for use in scanning of the PDMS channels, the focus position was determined at 6 locations in each channel. The second order fit and measured focus positions are shown in Figure 5.2.

The filled red circles indicate the measured data and the blue grid shows the best possible 3D polynomial fit to these points. Notice that the accuracy of the fit varies over the surface. At some locations the measured focus positions are exactly in the center of the blue grid lines, indicating a good fit. Other locations have a significant deviation between fit and actual focus position like the one indicated by the black arrow. The residual of the fit that is shown in Figure 5.2 was determined for each of the 36 measured focus positions. The results are shown in Figure 5.3.

Each channel is shown separately to illustrate the variations in fitting accuracy between different channels. For example, the first (black line, squares) and sixth (pink line, left pointing triangles) channels: The residuals are similar for the position at $18 \mathrm{~mm}$ along the cartridge. While at $12 \mathrm{~mm}$, there is a difference of $6.6 \mu \mathrm{m}$ between these two channels. The depth of field of the microscope objective, $40 \times / 0.6 \mathrm{NA}$, is indicated by the light gray band. It is clear that the deviation from a $2^{\text {nd }}$ order fit for the majority of the measured focus points is outside of this band. This means that the current method of interpolation cannot be used to scan the microstructure channels.

Since the variation between different channels is large, it is better to fit each channel separately. This was achieved by using a natural cubic spline interpolating function. This is a spline of the third degree and has $\mathrm{C}^{2}$ continuity. Meaning that the second derivative of the spline is zero at the endpoints of the interpolation interval. This is done to achieve a 'natural' extrapolation of the fit outside of the interpolation endpoints. Since the focus cannot be determined at the very ends of the channels, this is necessary to prevent the fit from deviating to much in these locations. A 


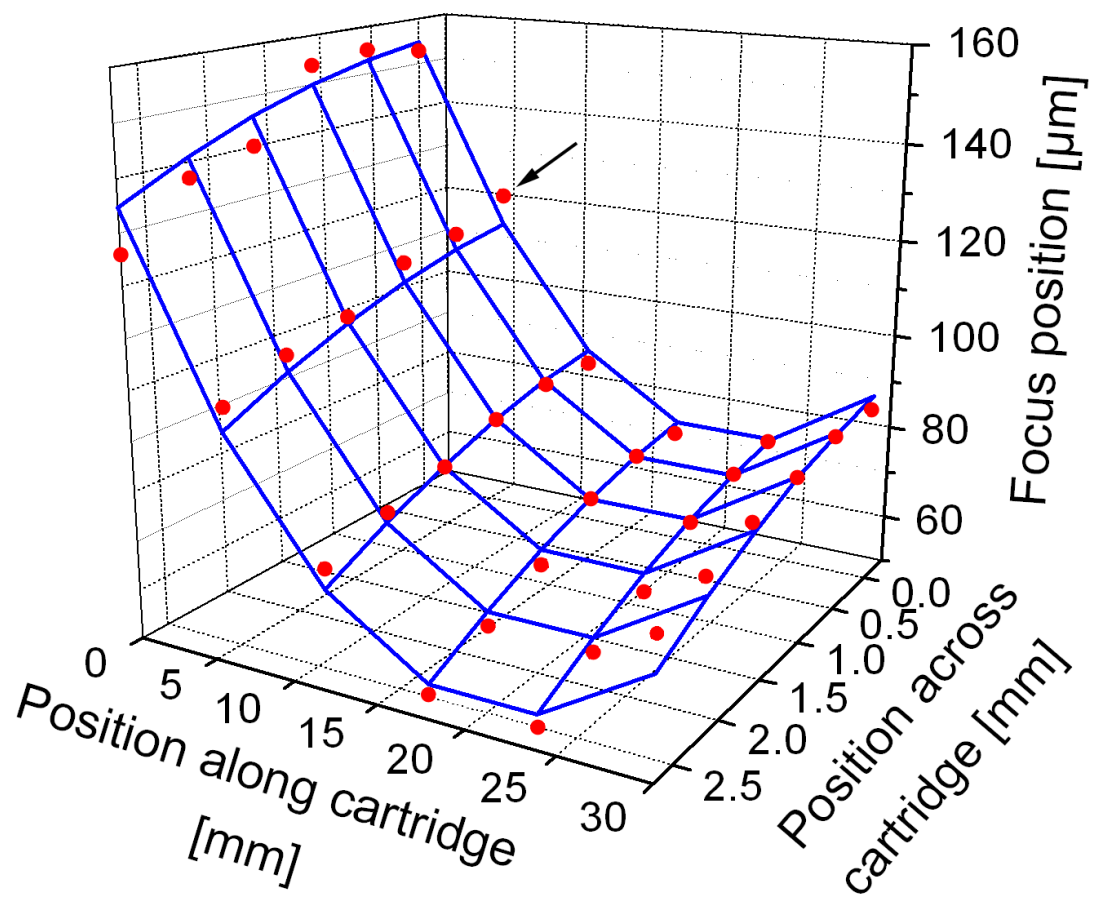

FIGURE 5.2 : 3D plot of a grid of 6 by 6 focus points that were determined using the bright-field autofocus method (Red circles). The x-axis (position across cartridge) and $\mathrm{y}$-axis (position along cartridge) have been given the same length in this plot for clarity. The blue colored grid indicates the results of a second order two dimensional fit to the measured focus points. The $\mathrm{R}^{2}$ of the fit is 0.98 . This suggests that the fit is relatively good. However, for some locations on the imaging surface, the deviation between measured and fitted focus position is too large. This would cause the cells in that area of the cartridge to be out of focus. The black arrow indicates such a location on the imaging surface were there is a significant fit error.

Labview script was written that fits a natural cubic spline to each channel separately. The results, using the same data points as in Figures 5.2 and 5.3, are shown in Figure 5.4.

The accuracy of the spline fit cannot be determined the same way as in the polynomial fit. The spline interpolation is designed to coincide with each focus position so the residuals will all be zero.

To determine the accuracy, the focus position was determined at intermediate points between the original locations. These intermediate points were compared to the spline fit and the results are shown in Figure 5.5. The light gray band again indicates the depth of field of the microscope objective at $580 \mathrm{~nm}$, which is $1.6 \mu \mathrm{m}$. The majority of the points are now within this band, indicating that the focus at intermediate points is accurately 


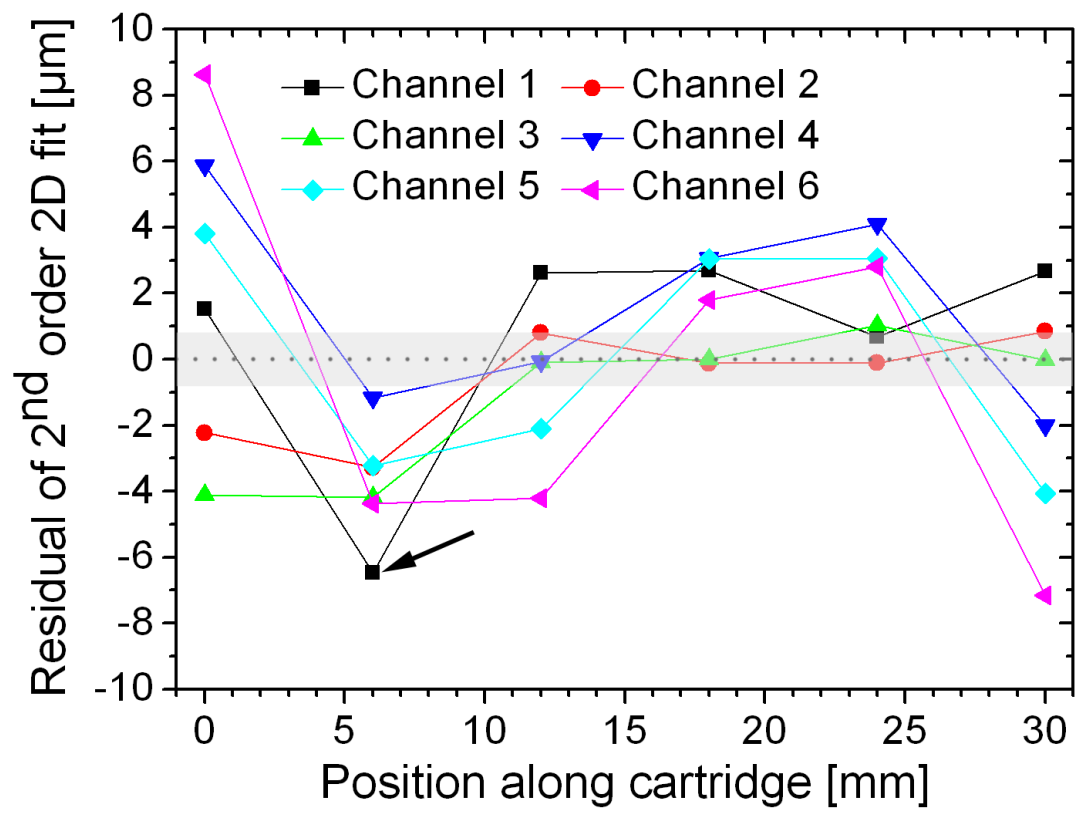

FiguRE 5.3 : Residual of $2^{\text {nd }}$ order $2 \mathrm{D}$ fit as shown in Figure 5.2. Each of the six channels is depicted by a different colored line with different icons. The black arrow indicates the same location in the first channel as also indicated in Figure 5.2. The standard deviation of all the residuals is $3.5 \mu \mathrm{m}$ and the range is -7.2 to $8.6 \mu \mathrm{m}$. The depth of field (DOF) of the microscope objective that is used in the CellTracks TDI is around $1.6 \mu \mathrm{m}$ for $580 \mathrm{~nm}$ (indicated by the light gray horizontal band). Therefore, the average error is over twice as large as the DOF, indicating that the $2^{\text {nd }}$ order $2 \mathrm{D}$ fit to the focus points cannot be used for scanning the microstructure channels.

determined using the natural spline fit. Therefore, a cartridge featuring the U-Groove microstructures can now be scanned in the CellTracks TDI system.

\subsubsection{CONTROL SOFTWARE}

After the focus position is determined using the autofocus method and interpolated, the sample is ready for scanning. To successfully scan a cartridge featuring the U-Groove microstructure, some changes to the CellTracks TDI system were necessary.

Normally, after a cartridge is first entered into the system, the borders are manually selected by the operator at 6 locations. These border locations are then used to calculate the center position of the chamber and the stages are set to home at that position. In case of the microstructures, it is more useful to set the home position of the stages to the first channel. 


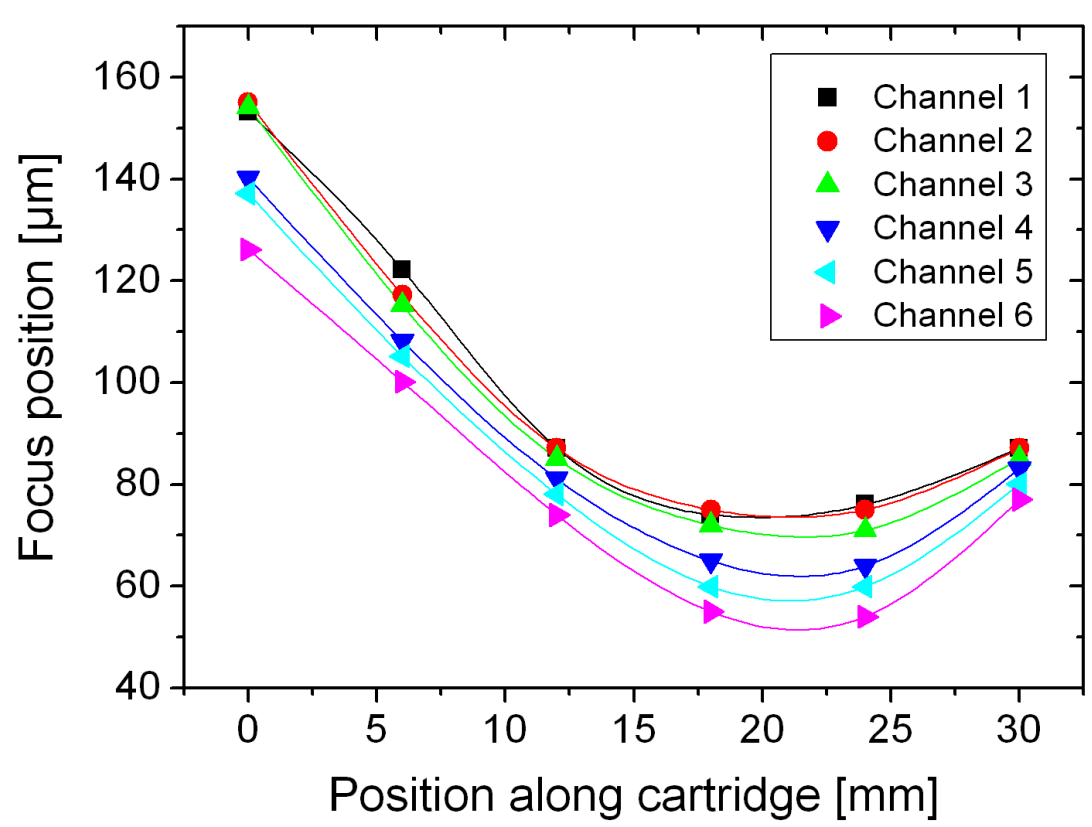

FIGURE 5.4 : Graph showing the results of a natural cubic spline fit to each groove separately. The fitted curves are smooth and the first and second order derivatives at the determined focus points are equal on both sides, preventing discontinuities. Also, if the focus cannot be determined at the exact edge of the channel, the spline fit is extrapolated using the same slope as present at the last data point (second order derivative is zero). This prevents large errors at the edges of the channels.

Therefore, the Labview program was adapted to let the user select the center of the first channel. Then, the home position of the stages is set to that location. Next, the focus position is determined along the six channels and a cubic spline is fitted to obtain intermediate focus positions for use during scanning.

In a scan of a cartridge with a glass surface, strips of $180 \mu \mathrm{m}$ wide and $30 \mathrm{~mm}$ long are scanned side by side to cover the entire imaging area. However, since the spacing of the U-Groove microstructures is $480 \mu \mathrm{m}$, this has to be changed. The offset between a scanned strip and the next was set to $473 \mu \mathrm{m}$. This is slightly lower than the design spacing of $480 \mu \mathrm{m}$ and is due to shrinking of the PDMS during curing (around 1.4\%, data not shown). Also, the number of strips to scan is reduced from 16 to 6 , as there are only 6 channels. After the first microstructure channel is scanned for the first color, the same channel is then scanned for the remaining fluorescent colors and for bright-field. This as opposed to a scan of a cartridge with a glass surface. Here, the entire surface is first scanned for one color and then the 


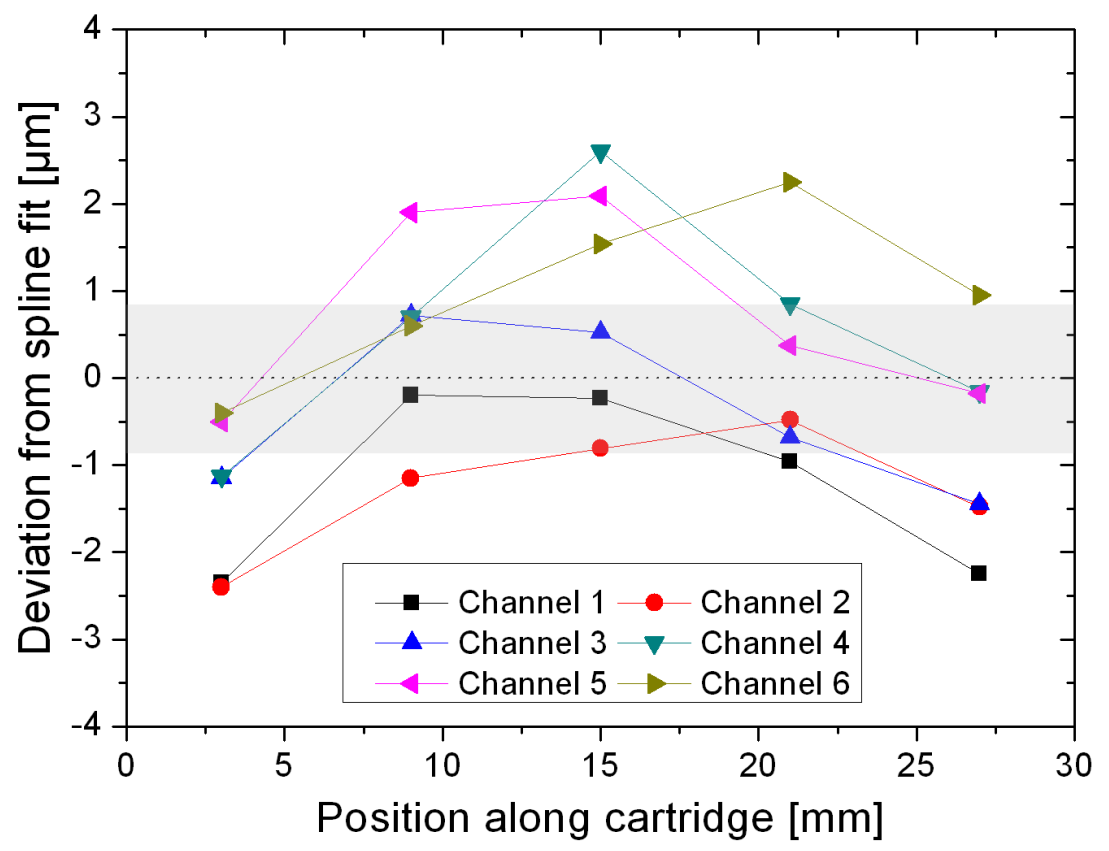

FiguRE 5.5 : Overview of the fit accuracy of the cubic spline fit for each of the six channels as shown in Figure 5.4. The focus position was determined at intermediate points between the ones used to fit the spline. The light gray band indicates the depth of field of the microscope objective, which is $1.6 \mu \mathrm{m}$ at $580 \mathrm{~nm}$.

entire surface is scanned for the remaining colors one by one. Reversing this order reduces possible scan errors due to cell movement inside the channels.

\subsubsection{IMPROVEMENTS IN SCAN AND ANALYSIS TIMES}

The use of the U-Groove microstructure brings improvements in both scanning and analysis times. The scan time is reduced because the number of strips that need to be scanned is reduced from 16 to 6 . This reduces the scan time from 38 to 14 minutes as shown in Table 5.1. However, due to the increase in required focus positions for the feedforward algorithm, the focusing time is increased from 2 to 4 minutes.

Improvements in analysis times are mainly due to the reduced amount of data that needs to be processed. The reduction in image data size as shown in the table is not the same as the reduction in the imaging area. This is due to the fact that the channels are $80 \mu \mathrm{m}$ wide whereas the illumination profile is $180 \mu \mathrm{m}$ wide. Images can be cropped after scanning to the channel width of $80 \mu \mathrm{m}$, however, the channels in the PDMS rarely run exactly straight from beginning to end, due to the flexibility of the PDMS. This 
TABLE 5.1 : Comparison of scanning and image analysis times in CellTracks TDI and CellTracks TDI with cell alignment microstructures. Image processing times were determined on a PC with an Intel Core2Duo 2.1Ghz CPU, 4 GB RAM and Windows XP 64-bit. Image processing was done using a collection of custom ImageJ macros.

\begin{tabular}{lll}
\hline Property & CellTracks TDI & $\begin{array}{l}\text { CellTracks TDI }+ \\
\text { alignment }\end{array}$ \\
\hline Imaging area & $81 \mathrm{~mm}^{2}$ & $14.4 \mathrm{~mm}^{2}$ \\
\hline Number of feed-forward focus points & 9 & 36 \\
Total focusing time & $2 \mathrm{~min}$ & $4 \mathrm{~min}$ \\
\hline Number of scans per color & 16 & 6 \\
Scan time, 4 colors & $38 \mathrm{~min}$ & $14 \mathrm{~min}$ \\
Total scan size (image data) & $16 \mathrm{~GB}$ & $6 \mathrm{~GB}$ \\
\hline Illumination and background correction & $44 \mathrm{~min}$ & $17 \mathrm{~min}$ \\
Event detection and gathering & $20 \mathrm{~min}$ & $9 \mathrm{~min}$ \\
Quantitative analysis and image montage & $1 \mathrm{~min}$ & $1 \mathrm{~min}$ \\
Total image processing time & $65 \mathrm{~min}$ & $27 \mathrm{~min}$ \\
\hline Total scan and processing time & $\mathbf{1 0 5} \mathbf{~ m i n}$ & $\mathbf{4 5} \mathbf{~ m i n}$ \\
\hline
\end{tabular}

means that the effective width of the images has to be larger than $80 \mu \mathrm{m}$.

The image processing consist of 3 sections. The first section corrects the raw image data for inhomogeneous illumination and subtracts the background. The time it takes to complete this section depends almost exclusively on the amount of raw image data present. For a scan of a cartridge with a glass imaging surface this is $\sim 44$ minutes and it is $\sim 17$ minutes for a cartridge with a microstructured imaging surface. The second section detects and combines events based on a fixed threshold in the PE fluorescence image. The analysis time for this part depends solely on the number of events. For this example, the number of events was assumed to be 234. This is the average number of $\mathrm{PE}$ positive events that were detected in scans of 91 samples from cancer patients [16]. However, this section also combines events that are on the border of two images. The time it takes to load additional images is dependent on the number of borders that a typical image has. In the case of a glass analysis surface, this is 4, because only 2 sides are considered at a time. For the U-Groove this is only 1 , either at the bottom or at the top of the image. Typical analysis times for this second section are 20 minutes and 9 minutes for the CellSearch and microstructured cartridges respectively.

The last section of the image analysis gathers quantitative parameters for each event, like total intensity, mean intensity, circularity and area. It also combines all fluorescent-, bright-field- and an overlay images side by side. This combined image is used during review to display the event under investigation to the reviewer. In general this last section of image analysis 

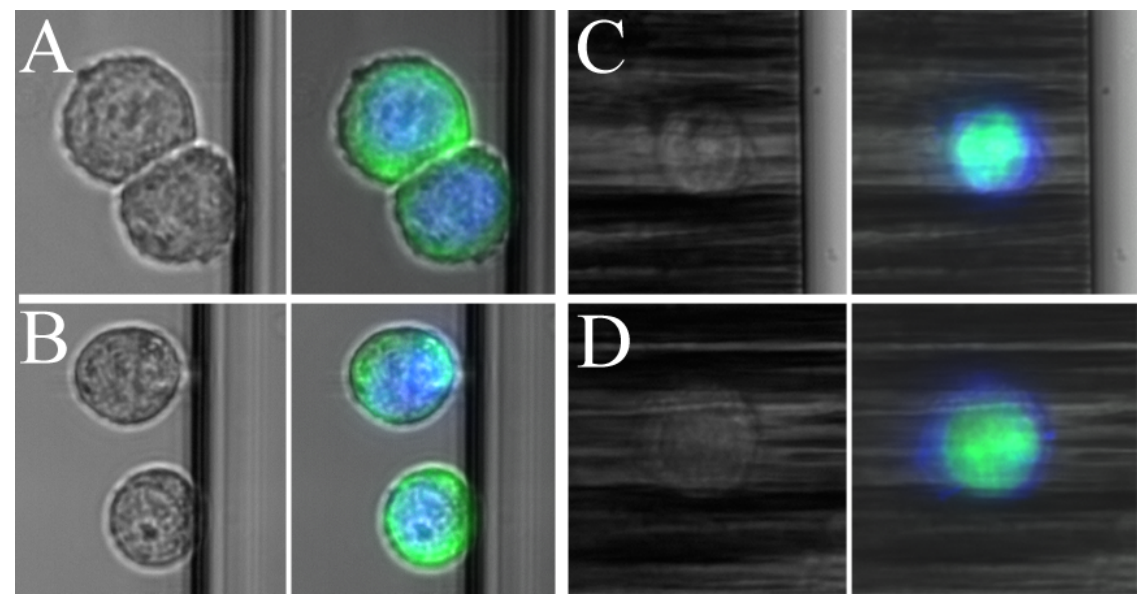

FigURE 5.6 : Overview of effect that unbound magnetic particles have on imaging of CTC in the U-Groove microstructures. (A and B) SKBR-3 cells in a channel of the U-Groove without unbound magnetic particles. (C and D) With unbound magnetic particles at a level that is common after preparation of a sample in the AutoPrep.

is the fastest and takes only 1-2 minutes.

In total, the combined scanning and image analysis time for a cartridge with a glass top and 234 events is 1 hour and 45 minutes. For a cartridge featuring the U-Groove microstructure this is 45 minutes. The image analysis time was determined on a PC running Windows XP (32 bit), with 4 GB RAM, a $2.1 \mathrm{GHz}$ Core2Duo CPU and ImageJ 1.43.

\subsubsection{IMAGE QUALITY COMPARISON WITH AND WITHOUT UNBOUND FERROFLUID}

Up to this point, the effect that unbound ferrofluid particles have on quantitative and qualitative imaging have not been discussed. To show their influence, we have imaged SKBR-3 cells in the U-Groove microstructure without unbound ferrofluid particles. Two examples of cells can be seen in Figure 5.6, panels A and B.

There is quite some detail visible in the bright-field image (left side) as well as in the overlay (right side) of the fluorescent channels and the brightfield image. Next, a sample containing around $40 \mu \mathrm{g} / \mathrm{ml}$ of unbound EpCAMFerrofluid was imaged. This amount of unbound ferrofluid corresponds to the amount that a typical sample has that is processed by the AutoPrep (Veridex, Raritan, NJ, USA). The differences are quite clear. Almost all detail is lost in the bright-field image and the fluorescence is scattered by the ferrofluid particles. 


\subsubsection{LINEARITY OF RECOVERY USING SKBR-3 CELLS}

The linearity of the recovery of SKBR-3 cells in the U-Groove microstructure was compared to a CellSearch cartridge with a glass imaging surface. For this experiment, six samples where used that contained varying numbers of SKBR-3 cells, ranging from $\sim 10$ to 2000 . All samples were washed three times to remove most of the free ferrofluid in the samples after magnetic labeling of the SKBR-3 cells.

All twelve samples, six from each cartridge type, were imaged using the CellTracks TDI system. Three channels were imaged: DAPI, to identify the nucleus, CK-PE, to identify the cytoskeleton and bright-field, to examine the morphology of the entire cell. All twelve scans were processed using the automatic image analysis routine written in ImageJ. The results were examined using a custom build Labview VI. Individual cells were counted based on the following criteria: Intact nucleus surrounded by the cytoskeleton on all sides and a round to oval morphology. Despite careful preparation of the samples, in $20 \%$ of all cases, two cells were clustered together and these show up as one event in the image analysis. Because these clustered cells most likely were already clustered before they entered the cartridge, they were counted as being one cell. The results of this experiment are shown in Figure 5.7. The linearity is good, with an $\mathrm{R}^{2}$ of 0.996 and a slope of 0.937 , in the clinically relevant range of $0-1000 \mathrm{CTC} / 7.5 \mathrm{ml}$ of blood.

\subsection{Discussion}

Cell analysis in image cytometry usually consists of scanning all or part of a sample that is located at an imaging surface. For most samples, scanning part of the sample surface is enough to get a statistically significant determination of the cell sub-populations. However, for cell populations that contain rare cells, like the sample processed by the CellSearch method, this is not the case. All cells in these samples need to be imaged to prevent not detecting the rare CTC. In Chapter 4 we discussed the development and testing of cell concentrating microstructures that reduce the scanning time for CTC samples significantly. Several types were tested, but the U-Groove microstructure was most suited to be implemented in the CellTracks TDI systems, which was discussed in Chapter 2.

This microstructure features six channels, $80 \mu \mathrm{m}$ in width and $30 \mathrm{~mm}$ in length, with a reduction in imaging surface area of 5.6 and in analysis time of approximately 3 times. They are fabricated using PDMS molding of etched wafer surfaces. As a result, the surfaces of these microstructures are smooth and this allows for a high capture efficiency of $\sim 99 \%$. Also, image quality is comparable to cells imaged in a standard CellSearch cartridge.

For the successful implementation of the microstructured cartridges in the CellTracks TDI system, some changes were necessary. A new autofocus algorithm was created that can be used to obtain the focus position inside 


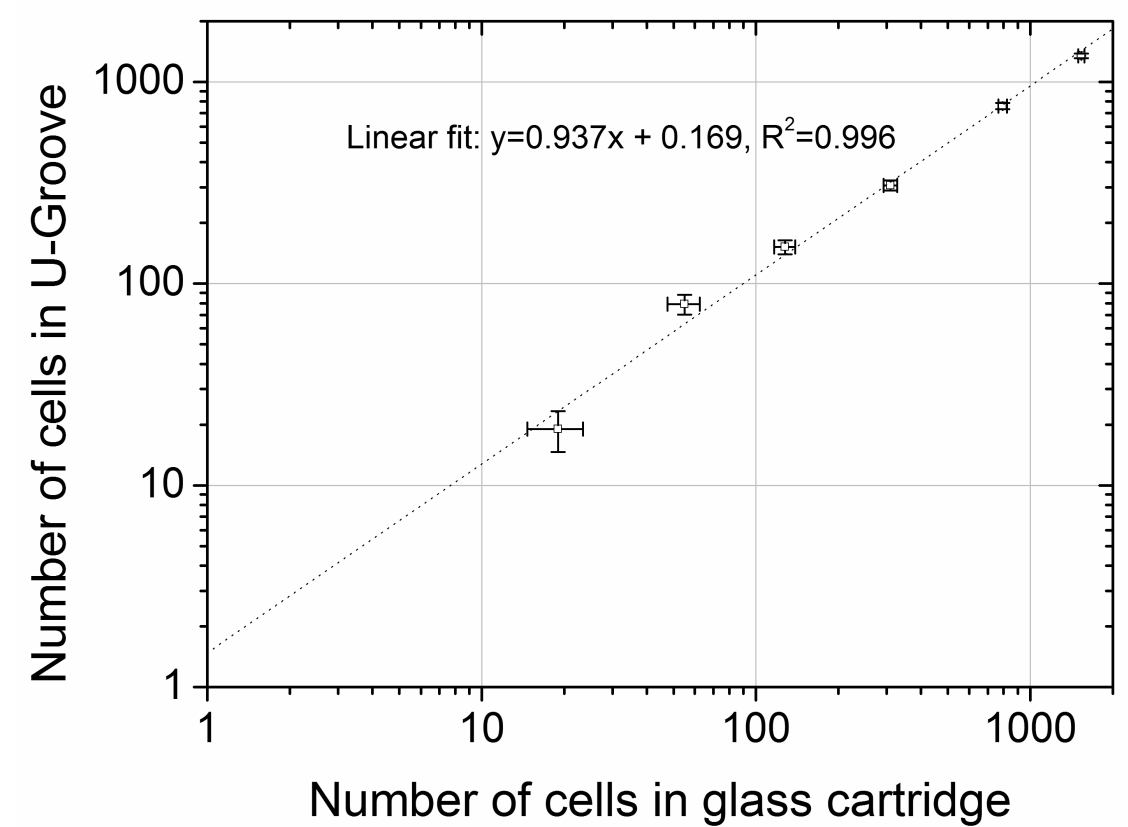

FIGURE 5.7 : Recovery of SKBR-3 cells in cartridges with a glass imaging surface and a U-Groove surface. The linearity is good for the clinically relevant range of $0-1000$ cells.

a channel of the U-Groove microstructure. It uses bright-field images that are integrated over the entire range of the microscope objective positioning system. This allows for the determination of the focus within the channels without using laser light that would otherwise bleach the cells in the sample. A new fit algorithm was used to interpolate the focus positions between the predetermined locations. The spline fit algorithm works remarkably better than the 3D polynomial fit that was used until that time in the CellTracks TDI system. This fit algorithm was subsequently incorporated in the system and used for all types of samples. An advantage related to the limited number of channels at the imaging surface is that the intermediate focus positions only need to be determined along those channels, since these are the only locations that will be imaged.

The Labview control software was also adapted to the use of the microstructured cartridges. It still uses manual control to select the location of the first channel, however this could be automated.

The fit accuracy as determined in Figure 5.5 is at some locations not within the depth of field of the microscope objective. This might be due to the focus determination method or due to the fact that not enough feed forward focus positions were chosen. We estimate the accuracy of the bright-field autofocus method to be around $0.5 \mu \mathrm{m}$. This could be improved 
by extending the 'combined TDI image' that is recored during autofocus determination. Also, the number of locations along a channel at which the focus is determined could be increased. However, this will increase the autofocusing time significantly.

The microstructures also cause a reduction in imaging and analysis times. The time it takes to image the entire analysis surface is reduced by a factor of 2.7 , while the image analysis time was reduced by a factor of 2.4. Image analysis times could be easily reduced further by the use of the latest multi-core computers. We estimate that a 4 -fold reduction would be feasible.

Some secondary effects due to the use of the microstructures where also observed. They prevent the sideways movement of cells across the top of the cartridge in samples with low amounts of unbound ferrofluids. This is mainly due to the small component of the magnetic field that exists parallel to the imaging surface. In standard patient samples that are processed by the AutoPrep, the level of unbound ferrofluids still present in the sample prevents all sideways movement. Therefore, this phenomenon is usually not observed. A second effect is that, while concentrating the cells, the channels also concentrate the unbound ferrofluids in the sample at the analysis surface. At low levels this is not an issue. The autofocus method works up to concentrations of $2 \mu \mathrm{g} / \mathrm{ml}$. However, the average concentration of unbound ferrofluid in a sample is $40 \mu \mathrm{g} / \mathrm{ml}$. Thereby it is necessary to remove most of these ferrofluids from a standard AutoPrep sample before it can be imaged using the microstructured cartridges. Also, due to the permeability of PDMS for various gases, formation of air bubbles occurs in the sample if they are kept for more than a day. Although this period may be prolonged by keeping those sample chambers in an environment with high humidity to prevent dehydration of the sample chamber.

The microstructured cartridges were successfully integrated into the CellTracks TDI setup. However, some optimizations are still necessary to benefit of their true potential. 


\subsection{REFERENCES}

[1] M. Cristofanilli, T. Budd, M. Ellis, A. Stopeck, J. Matera, M. Miller, J. Reuben, G. Doyle, W. Allard, L. Terstappen, and D. Hayes, "Circulating tumor cells, disease progression, and survival in metastatic breast cancer," $N$ Engl J Med, vol. 351(8), pp. 781-791, 2004.

[2] J. Gaforio, M. Serrano, P. Sanchez-Rovira, A. Sirvent, M. Delgado-Rodriguez, M. Campos, N. de la Torre, I. Algarra, R. Dueñas, and A. Lozano, "Detection of breast cancer cells in the peripheral blood is positively correlated with estrogenreceptor status and predicts for poor prognosis," Int J Cancer, vol. 107(6), pp. 984990, 2003.

[3] J. De Bono, H. Scher, R. Montgomery, C. Parker, M. Miller, H. Tissing, G. Doyle, L. Terstappen, K. Pienta, and D. Raghavan, "Circulating tumor cells predict survival benefit from treatment in metastatic castration resistant prostate cancer," Clin Can Res, vol. 14(19), pp. 6302-6309, 2008.

[4] S. Cohen, C. Punt, N. Iannotti, B. Saidman, K. Sabbath, N. Gabrail, J. Picus, M. Morse, E. Mitchell, C. Desch, M. Miller, G. Doyle, H. Tissing, L. Terstappen, and N. Meropol, "The relationship of circulating tumor cells to tumor response, progression-free survival, and overall survival in patients with metastatic colorectal cancer," Journal of Clinical Oncology, vol. 26(19), pp. 3213-3221, 2008.

[5] M. Liu, P. Shields, R. Warren, P. Cohen, M. Wilkinson, Y. Ottaviano, S. Rao, J. Eng-Wong, F. Seillier-Moiseiwitsch, A. Noone, and C. Isaacs, "Circulating tumor cells: A useful predictor of treatment efficacy in metastatic breast cancer," Journal of Clinical Oncology, vol. 27(31), pp. 5153-5159, 2009.

[6] J. Tol, M. Koopman, M. Miller, A. Tibbe, A. Cats, G. Creemers, A. Vos, I. Nagtegaal, L. Terstappen, and C. Punt, "Circulating tumour cells early predict progression-free and overall survival in advanced colorectal cancer patients treated with chemotherapy and targeted agents," Annals of Oncology, vol. 21(5), pp. 1006-1012, 2010.

[7] P. Liberti, C. Rao, and L. Terstappen, "Optimization of ferrofluids and protocols for the enrichment of breast tumor cells in blood," Journal of Magnetism and Magnetic Materials, vol. 225(1-2), pp. 301-307, 2001.

[8] P. Liberti, Y. Wang, W. Tang, B. Feeley, and D. Gohel, "Apparatus and methods for magnetic separation featuring external magnetic means," USA Patent 5,466,574, 1995.

[9] F. Schreuder, Laser image cytometer for analysis of circulating tumor cells. PhD thesis, PhD Thesis University of Twente, ISBN 9789036526104, 2008.

[10] T. Scholtens, F. Schreuder, S. Ligthart, J. Swennenhuis, A. Tibbe, J. Greve, and L. Terstappen, "Celltracks TDI: An image cytometer for cell characterization," Cytometry Part A, vol. 79(3), pp. 203-213, 2011.

[11] A. Tibbe, Cell Tracks: The next step in cell analysis. PhD thesis, PhD Thesis University of Twente, ISBN 9789036515416, 2001.

[12] A. Tibbe, B. de Grooth, J. Greve, P. Liberti, G. Dolan, and L. Terstappen, "Optical tracking and detection of immunomagnetically selected and aligned cells," Nature Biotechnology, vol. 17, pp. 1210-1213, 1999.

[13] W. Rasband, "Imagej," http://rsbweb.nih.gov/ij/index.html, vol. -, pp. -, 1997 2010 .

[14] N. Otsu, "Threshold selection method from gray-level histograms," IEEE Trans Syst Man Cybern, vol. SMC-9(1), pp. 62-66, 1979. 
[15] P. Liao, T. Chen, and P. Chung, "A fast algorithm for multilevel thresholding," Journal of Information Science and Engineering, vol. 17(5), pp. 713-727, 2001.

[16] T. Scholtens, F. Schreuder, S. Ligthart, J. Swennenhuis, A. Tibbe, J. Greve, and L. Terstappen, "Automated identification of circulating tumor cells by image cytometry," Cytometry Part A, vol. 81(2), pp. 138-148, 2012. 


\section{QUANTITATIVE AND QUALITATIVE EFFECT OF FREE FERROFLUID ON CELL ANALYSIS}

The CellSearch system is a validated system for the detection of Circulating Tumor Cells (CTC). Ferrofluid labeled with antibodies directed against the Epithelial Cell Adhesion molecule (EpCAM) is used to enrich CTC. Ferrofluid is added in excess to drive the kinetics of binding target cells. As a result, free ferrofluid, without binding to cells, is present in the final sample after enrichment. Here we investigate the influence of free ferrofluid on bright-field- and fluorescence imaging.

Different concentrations of free ferrofluid were added to magnetically and fluorescently labeled beads, T-lymphocytes and cells from the breast cancer cell line SKBR-3 to determine the effects on fluorescence- and bright-field imaging as measured on the CellTracks TDI system.

At a free ferrofluid concentration of $40 \mu \mathrm{g} / \mathrm{ml}$ in the final sample, used in the CellSearch system to enrich CTC, the fluorescence intensity of the beads and cells is reduced by $11-67 \%$ and the coefficient of variation increased 1.5-6 fold. The effects are most pronounced in the blue region (400-460 nm). Reduction of free ferrofluid from $40 \mu \mathrm{g} / \mathrm{ml}$ to below $2 \mu \mathrm{g} / \mathrm{ml}$ is needed to prevent reduction of fluorescent signals and enable the identification of the cytoplasmic membrane by image analysis of the bright-field images.

A $95 \%$ reduction of free ferrofluid after CTC enrichment on the CellTracks AutoPrep is needed to permit the identification of the cell surface by bright-field and enable unencumbered detection of fluorescence signals from the cells. 


\subsection{INTRODUCTION}

In the CellSearch ${ }^{\circledR}$ system, ferrofluids coated with anti-EpCAM antibodies are used to immunomagnetically enrich Circulating Tumor Cells (CTC) from $7.5 \mathrm{ml}$ of blood. Most of the ferrofluid is not bound to the cell surface and will remain in solution. In the CellTracks AutoPrep ${ }^{\circledR}$, various magnetic separation steps are used to enrich and concentrate the CTC [1]. During these steps, the volume is reduced from $7.5 \mathrm{ml}$ of blood to $370 \mu \mathrm{l}$ of a cell suspension containing the majority of CTC expressing EpCAM, 850-5200 leukocytes, virtually no erythrocytes or platelets and $\sim 50 \%$ of the amount of ferrofluid used for enrichment. The reason that not all ferrofluid is retained in the sample is that in the last preparation step the system allows the cells to settle and aspirates and discards the top layer that contains a portion of the ferrofluid. After transfer of the sample into the CellSearch cartridge and placement into the Magnest, the magnetically labeled cells and free ferrofluid move to the imaging surface $[2,3]$. The free ferrofluids can now be observed as a layer of ferrofluid lines that are created according to the local magnetic field. This layer of ferrofluid causes distortions in imaging of the cells, both in bright-field and in fluorescence imaging. The loss of fluorescence in combination with the non-homogeneous distortion to imaging can introduce errors in the classification of cells. Here we investigate the quantitative and qualitative effects of free ferrofluid on the ability to detect the cell membrane by means of bright-field images and the quantitative analysis of cell antigens by fluorescence.

\subsection{Materials And Methods}

\subsubsection{FLUORESCENT MAGNETIC BEADS}

Three types of fluorescent magnetic beads were used to evaluate the influence of free ferrofluid on the fluorescent signals: glacial blue, envy green and flash red (Compel magnetic microspheres, Bangs Laboratories Inc., Fishers, IN, USA). These beads have an average diameter of $8 \mu \mathrm{m}$, which is similar to the average diameter of a normal blood cell. The spectra of the beads are shown in Table 6.1 and are comparable to the three fluorescent dyes used in the CellSearch system to detect CTC. The spectrum of glacial blue beads is close to 4',6-diamidino-2-phenylindole (DAPI) used to stain the cell nucleus, envy green beads is close to R-Phycoerythrin (PE) conjugated antibodies recognizing cytokeratins specific for epithelial cells and flash red beads is close to Allophycocyanin (APC), conjugated to antibodies recognizing CD45 specific for leukocytes.

The coefficient of variation $(\mathrm{CV})$ of the fluorescence intensity is however relatively high $(>6 \%)$ and prevents an accurate quantification of the effect of low EpCAM-Ferrofluid concentrations on the fluorescence signals derived from the beads. To circumvent this problem, the green and red beads 
TABLE 6.1 : Overview of excitation and emission maxima of the three bead types and their corresponding fluorescent dyes used in the CellSearch system.

\begin{tabular}{lll}
\hline Fluorochrome & Excitation maximum & Emission maximum \\
\hline Glacial Blue & 360 & 450 \\
DAPI & 358 & 461 \\
\hline Envy Green & 525 & 565 \\
R-Phycoerythrin & 565 & 576 \\
\hline Flash Red & 660 & 690 \\
Allophycocyanin & 650 & 660 \\
\hline
\end{tabular}

were sorted with a FACSCalibur (BD Biosciences, San Jose, CA, USA) Flow Cytometer (FCM) and the blue beads with a FACSAria II FCM (BD Biosciences, San Jose, CA, USA). The latter instrument is equipped with a UV laser that can be used to excite the blue beads. The beads were sorted based on a narrow region on the histogram of the fluorescence intensity of each bead type. The results were verified by reanalysis of a portion of the sorted beads on the flowcytometer. The CV of the sorted beads was between 3 and 4\%. The collected bead samples were centrifuged at $300 \mathrm{~g}$ for 10 minutes and washed using Phosphate Buffered Saline (PBS). The final concentration of the blue, green and red bead solutions was approximately 100 beads $/ \mu$ l. To determine the effect of free ferrofluid on fluorescent beads, we created 10 samples with a volume of $370 \mu \mathrm{l}$ each, with increasing concentrations of free ferrofluid for the 3 bead types. For each sample $35 \mu \mathrm{l}$ of the bead solution was added to $0,0.74,1.85,3.7,7.4,18.5,37,74,185$ and $335 \mu \mathrm{l}$ of $200 \mu \mathrm{g} / \mathrm{ml}$ EpCAM-Ferrofluid (Veridex, Raritan, NJ, USA) and filled to $370 \mu \mathrm{l}$ with PBS. The final concentrations of free ferrofluid were $0,0.4,1,2,4,10,20,40,100$ and $181 \mu \mathrm{g} / \mathrm{ml}$ respectively. After preparation, the samples were transferred to a CellSearch cartridge and inserted into the Magnest. Samples were prepared in such a way that the magnetic incubation time in the Magnest was 15 minutes for all samples to prevent effects of different magnetic incubation times.

\subsubsection{LEUKOCYTES}

To determine the effect of free ferrofluid on nuclear fluorescence of leukocytes, a cell population is preferred with a relatively low $\mathrm{CV}$ of the fluorescence signals and a relatively homogeneous nucleus. In addition, cells need to be magnetically labeled to be able to move to the analysis surface of the CellSearch cartridge. To obtain such cells, blood was drawn from a healthy donor into a $10 \mathrm{ml}$ CellSave Preservative tube (Veridex, Raritan, NJ, USA) and left overnight. The next day, $300 \mu \mathrm{l}$ of blood was incubated with $15 \mu \mathrm{l}$ CD3-Ferrofluid at a concentration of $0.655 \mathrm{mg} / \mathrm{ml}$ (Veridex, Raritan, NJ, 
USA) and $5 \mu$ l Hoechst 33342 at a concentration of $1 \mathrm{mg} / \mathrm{ml}$ (Invitrogen, Carlsbad, CA, USA). The sample was incubated in the dark for 25 minutes at room temperature. Next, the red blood cells were lysed using FACSLysing solution (BD Biosciences, San Jose, USA) by adding 2,9 $\mathrm{ml}$ of MilliQ water to $320 \mu \mathrm{l}$ of $10 \times$ FACSLysing solution and adding this to the stained whole blood. After incubation for 10 minutes at room temperature in the dark the sample was washed to remove free dye and ferrofluid. To this end, the sample was centrifuged at $300 \mathrm{~g}$ for 5 minutes and the supernatant was aspirated. The pellet was then resuspended in $1 \mathrm{ml}$ PBS-AC. To obtain a sample enriched for T-lymphocytes without free ferrofluid, the samples were sorted on a FACSAria II FCM. Sorting criteria included the typical forward and side scatter properties of lymphocytes and the Hoechst fluorescence. Approximately $1 \times 10^{5}$ of these cells were obtained and a small fraction was reanalyzed to confirm that the cells were sorted correctly. To determine the effect of free ferrofluid on T-lymphocytes, 10 samples were made consisting of $60 \mu \mathrm{l}$ of the sorted lymphocytes $(100 \mathrm{cells} / \mu \mathrm{l})$ and $0,0.74,1.85,3.7,7.4$, 18.5, 37, 74, 185 and $310 \mu \mathrm{l}$ of $200 \mu \mathrm{g} / \mathrm{ml}$ EpCAM-Ferrofluid. The samples were diluted with PBS-AC to a final volume of $370 \mu \mathrm{l}$ resulting in final concentrations of free ferrofluid of $0,0.4,1,2,4,10,20,40,100$ and 168 $\mu \mathrm{g} / \mathrm{ml}$ respectively. After preparation, the samples were transferred to a CellSearch cartridge and inserted into the Magnest. To avoid differences due to different exposure times to the magnetic field, each sample was placed for 30 minutes in the Magnest before image acquisition in the CellTracks TDI.

\subsubsection{CANCER CELLS}

Cells from the breast cancer cell line SKBR-3 (ATCC, Middlesex, UK) were used as a model for CTC in cancer patients. The cells were cultured in RPMI 1640 Glutamax-I medium (Invitrogen, Carlsbad, CA, USA). Cells were harvested and subsequently fixed using paraformaldehyde (PFA) at a concentration of $1 \%$ for at least 30 minutes. The cells were washed twice using PBS after centrifugation at $300 \mathrm{~g}$ for 5 minutes. This resulted in a final sample of $1.5 \mathrm{ml}$ containing $4 \times 10^{6}$ cells $/ \mathrm{ml}$. The cells were incubated with EpCAM-Ferrofluid at a concentration of $80 \mu \mathrm{g} / \mathrm{ml}$ for 30 minutes. To obtain a sample of magnetically labeled SKBR-3 cells without free ferrofluid the samples were sorted on a FACSAria II FCM. Sorting criteria included the forward and side scatter properties and Hoechst fluorescence. To determine the effect of free ferrofluid on SKBR-3 cells, 10 samples were made consisting of $1 \mu \mathrm{l}$ of the sorted cells $(4000$ cells $/ \mu \mathrm{l})$ and $0,0.74,1.85$, $3.7,7.4,18.5,37,74,185$ and $364 \mu \mathrm{l}$ of $200 \mu \mathrm{g} / \mathrm{ml}$ EpCAM-Ferrofluid. The samples were diluted with PBS-AC to a final volume of $370 \mu \mathrm{l}$ resulting in final concentrations of free ferrofluid of $0,0.4,1,2,4,10,20,40,100$ and $197 \mu \mathrm{g} / \mathrm{ml}$ respectively. After preparation, the samples were transferred to a CellSearch cartridge and inserted into the Magnest. To avoid differences due 
to different exposure times to the magnetic field, each sample was placed for 30 minutes in the Magnest before image acquisition in the CellTracks TDI.

\subsubsection{CELLTRACKS TDI}

The CellTracks TDI system is a laser scanning image cytometer described in detail elsewhere $[4,5]$. Briefly, it uses a TDI camera to acquire images of cells while they are scanned at a constant velocity. Fluorescent labels are excited using 375, 491 and $639 \mathrm{~nm}$ lasers, and a high power LED underneath the sample is used to create bright-field images. All three laser lines are combined into an overlapping bundle and focused into an approximately square profile, $180 \times 180 \mu^{2}$ in size, using a beam homogenizing system. A $40 \times / 0.6 \mathrm{NA}$ objective is used to scan the cells that are collected at the imaging surface. To cover the entire imaging area of a cartridge, 16 scans, each $180 \mu \mathrm{m}$ wide, are made. This is repeated for each additional color that needs to be imaged. At a standard imaging speed of $1 \mathrm{~mm} / \mathrm{sec}$, each scan of the entire surface takes approximately 9 minutes, including overhead time. Raw images that are obtained by the CellTracks TDI system are processed and analyzed using the image analysis program ImageJ [6]. The background of all fluorescence images is removed and then they are corrected for inhomogeneous illumination by multiplying each pixel column with a predefined correction factor. Next, images are stitched together in groups of four to reduce the number of events that are on the border of an image.

\subsubsection{EVENT DETECTION IN CELLTRACKS TDI IMAGES}

To identify the glacial blue beads, leukocytes and SKBR-3 cells, thresholding on the DAPI channel was used, for identification of the envy green beads the $\mathrm{PE}$ channel and for the flash red beads the APC channel. A fixed threshold is used on the fluorescence image. Each event that is detected is extracted from the original image, for all colors, and stored in a separate multipage TIFF file that combines all detected events. Extraction of events is done using a fixed window of $30 \times 30 \mu \mathrm{m}^{2}$, which is centered on the intensity-weighted center of the detected events. After all events have been extracted from the original images, quantitative fluorescence and morphological parameters are extracted using an automated Otsu threshold [7, 8]. Typical fluorescent parameters include mean and total intensity and the ratios between the different colors. Finally, the events are reviewed using a custom built Labview program. This program displays the quantitative information of the events in dot plots and histograms and displays the fluorescence and bright-field images from each selected event. For the analysis of the images from beads, T-lymphocytes and SKBR-3 cells the Labview analysis program was adapted to enable the selection of single cells and beads. The area, circularity and location of the detected events are used to discriminate 
single cells from cell clusters and debris. Single cells were defined as events with a circularity between $0.84-0.92$ and an area between $60-120 \mu^{2}$. Additional criteria to correctly identify single cells are needed because a relatively low fixed threshold is used to select the events of interest from the acquired images. Therefore, some events that are selected will still contain clusters of 2 or more cells. During determination of quantitative parameters (e.g. total intensity), an Otsu threshold is used. When a cluster of 2 cells is present in the selected window of $30 \times 30 \mu \mathrm{m}^{2}$, neither center of mass of these cells will be located in the middle of the window. Therefore, events for which the center of mass is not located within $1 \mu \mathrm{m}$ of the center of the selection window were determined not to be single cells.

\subsection{Results}

\subsubsection{EFFECTS OF FREE FERROFLUID ON FLUORESCENCE MEASUREMENTS}

Figure 6.1 shows the transmission spectrum of $\mathrm{Fe}_{3} \mathrm{O}_{4}$, as measured with a UV-2401PC spectrophotometer (Shimadzu, Den Bosch, Netherlands) in combination with the emission spectra of the DAPI, PE and APC fluorescent dyes. The figure shows that a decrease in fluorescence yield can be expected for the dyes DAPI, PE and APC used in the CellSearch system. The effect on DAPI and therefore imaging of nuclear fluorescence is expected to be most profound.

To quantify the effect of free ferrofluid on fluorescent imaging, different concentrations of ferrofluid were added to blue, green and red beads. In total 10 concentrations were tested ranging from 0 to $181 \mu \mathrm{g} / \mathrm{ml}$. The typical concentration of free ferrofluid after processing $7.5 \mathrm{ml}$ of blood with the CellTracks AutoPrep of $40 \mu \mathrm{g} / \mathrm{ml}$ was included as well as two higher and seven lower concentrations. Samples were scanned on the CellTracks TDI system for bright-field and blue, green or red fluorescence for blue, green and red beads respectively.

The effect of free ferrofluid on the fluorescence intensity of blue beads is shown in Figure 6.2A, for green beads in Figure $6.2 \mathrm{~B}$ and for red beads in Figure 6.2C. Each panel (A-C) shows the effect of increasing concentrations of free ferrofluid (left to right) on the total fluorescence intensity (square markers, left y-axis) and the coefficient of variation of the fluorescence intensity (triangle markers, right y-axis). In Figure 6.2D the influence of free ferrofluid on the fluorescence intensity of all three beads is illustrated by normalization of the fluorescence based on the maximum intensity of each bead type. Up to a concentration of $4 \mu \mathrm{g} / \mathrm{ml}$ of free ferrofluid, no significant effect of the free ferrofluid is noticed. With higher concentrations, the average total intensity steadily drops and the CV increases. At a concentration of $40 \mu \mathrm{g} / \mathrm{ml}$ the average total intensity has dropped to 62,88 and $89 \%$ of the maximum intensity for the blue, green 


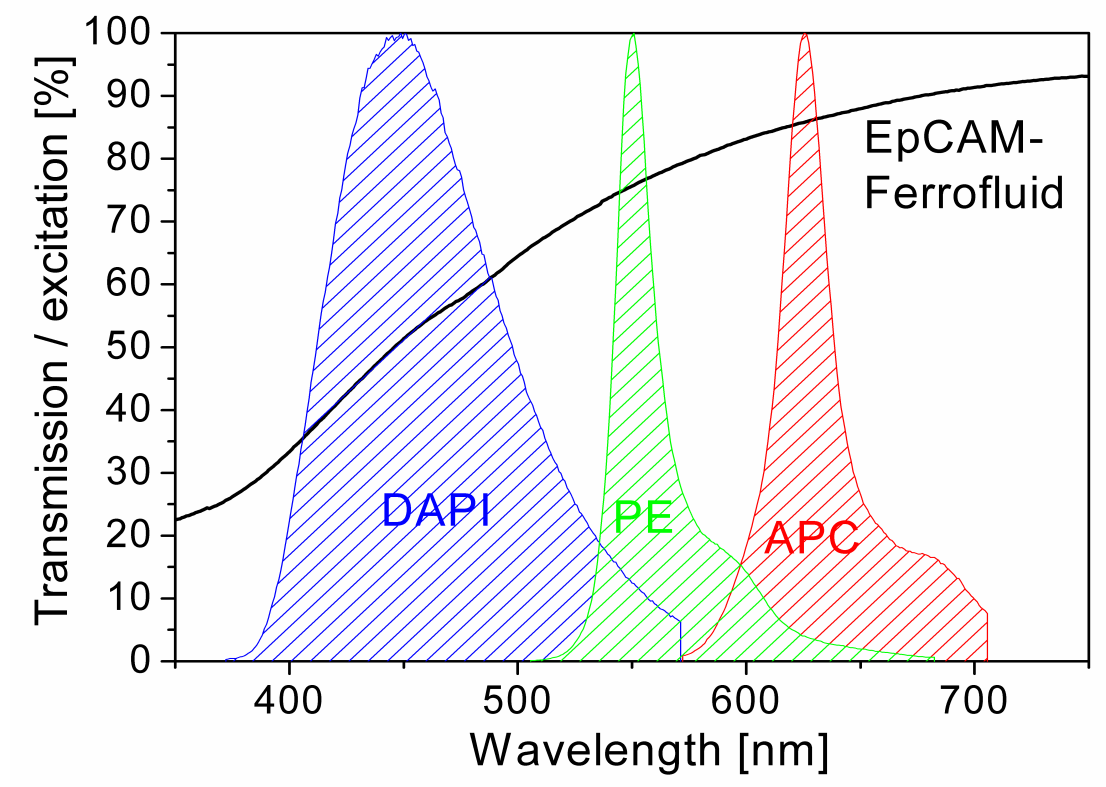

Figure 6.1 : Transmission spectra for EpCAM-Ferrofluid and excitation spectra for the three main fluorescent dyes that are used in the CellSearch system. The transmission spectrum was determined using a CellSearch cartridge, containing a sample of $40 \mu \mathrm{g} / \mathrm{ml}$, inserted into a Magnest. Excitation spectra were taken from the literature.

and red beads respectively. The effect of free ferrofluid on quantitative fluorescence determination is most pronounced with the blue beads. This is likely due to the absorption spectrum of $\mathrm{Fe}_{3} \mathrm{O}_{4}$, the main component of ferrofluid, which is highest in the blue region. At concentrations above $40 \mu \mathrm{g} / \mathrm{ml}$, the distribution of the aggregated free ferrofluid at the imaging surface is visually not homogeneous. Some parts of the imaging surface, most notably at the beginning of the analysis chamber at which the sample is introduced, show clusters of aggregated ferrofluid particles with areas in-between that have almost no ferrofluid aggregates. This is most likely due to in-homogeneity in the magnetic field of the CellTracks Magnest at that location or due to residual movement in the sample after introduction into the sample chamber.

\subsubsection{EFFECTS OF FREE FERROFLUID ON IMAGING OF LEUKOCYTES}

T-lymphocytes labeled with CD3 ferrofluids and void of free ferrofluid were obtained by sorting lymphocytes based on light scatter properties and Hoechst fluorescence. Before placement in the sample chamber, free ferrofluid was added at different concentrations, ranging from 0 to 168 $\mu \mathrm{g} / \mathrm{ml}$. The samples were placed in the CellTracks Magnest and scanned 
A
$B$

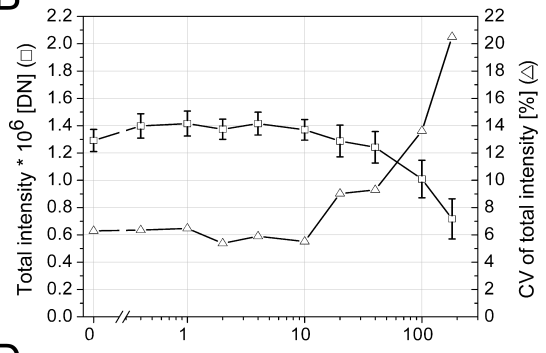

$\mathrm{D}$

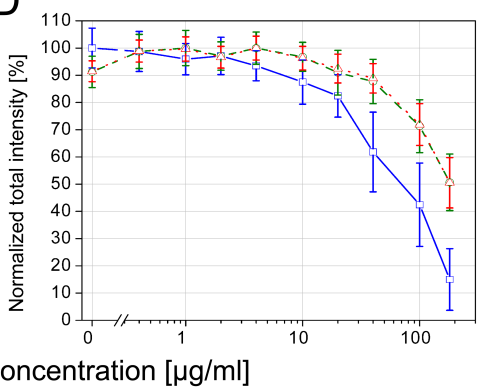

FiguRE 6.2 : Effect of free ferrofluid on quantitative fluorescence of three different bead types. (A-C) Squares: mean of total intensity of $\sim 1000$ beads with error bars showing standard deviation, left y-axis. Triangles: CV of total intensity, right y-axis. (A) Glacial Blue beads with excitation and emission spectra similar to DAPI, (B) Envy Green beads with spectra similar to PE and (C) Flash Red beads with spectra similar to APC. (D) Comparison of normalized total intensity based on the maximum value for the three different bead types. Squares/blue: Glacial Blue beads. Circles/green: Envy Green beads. Triangles/red: Flash Red beads.

on the CellTracks TDI system for bright-field and Hoechst fluorescence. In Figure 6.3 the Hoechst fluorescence intensity and its coefficient of variation are shown for the T-lymphocytes. The total intensity for a single cell is determined by summing all pixel values within a radius of $6 \mu \mathrm{m}$ from the center of the cell. The number of T-lymphocytes measured in the 10 experiments ranged from 222 - 1240 (mean 738, SD 377).

Similar to the measurements of fluorescent beads, the fluorescence intensity of the T-lymphocytes decreased and the coefficient of variation of the fluorescence increases when ferrofluid concentrations exceed $4 \mu \mathrm{g} / \mathrm{ml}$. The total intensity of a sample containing $40 \mu \mathrm{g} / \mathrm{ml}$ of free ferrofluid is $33 \%$ of a sample containing no free ferrofluid. When all free ferrofluid would be removed from a sample, the effective fluorescence intensity would therefore triple and the $\mathrm{CV}$ of the fluorescence would decrease from $40 \%$ to $7 \%$. In Figure 6.4 the effects of free ferrofluid on the fluorescence and bright-field images of T-lymphocytes are shown.

The bright-field images in the figure show the horizontally striped, barcode-like, pattern of the aggregated ferrofluids. They are aligned along 


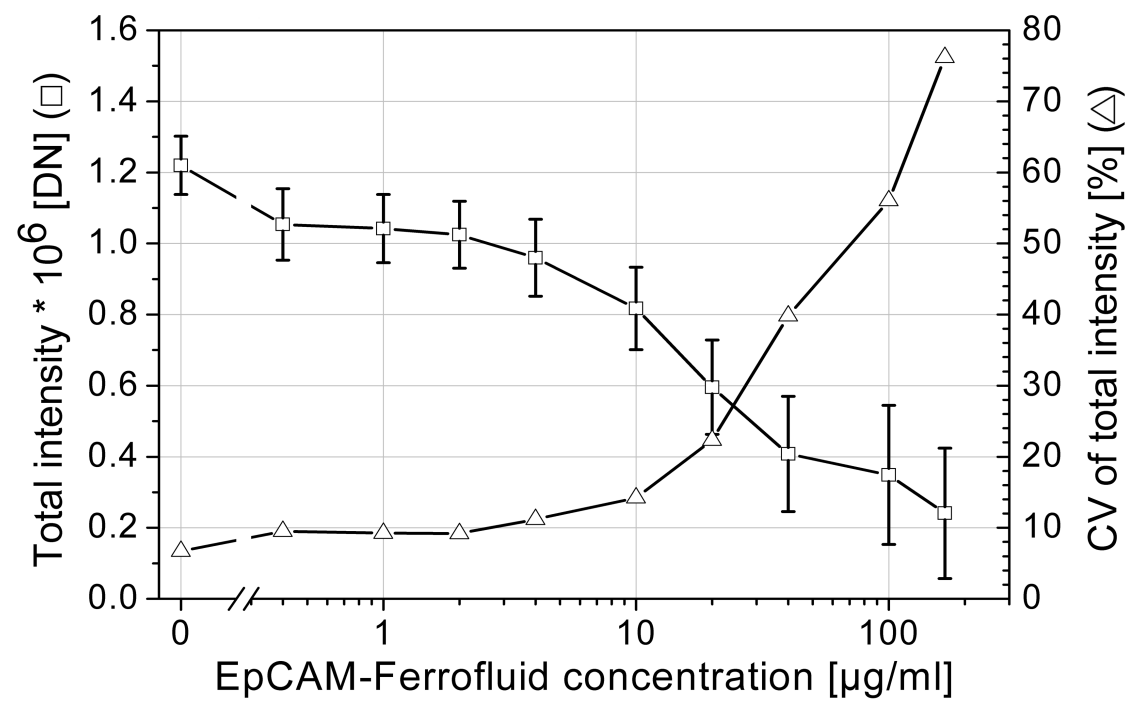

FiguRE 6.3 : Effect of free EpCAM-Ferrofluid on the quantitative nuclear fluorescence detection from T-lymphocytes. The line with square markers (left y-axis) indicates average total Hoechst fluorescence intensity and the corresponding standard deviation shown as an error-bar. The line with triangular markers shows the CV of the fluorescence intensity on the right y-axis.

the magnetic gradient resulting from the design of the magnets in the CellTracks Magnest [3]. The individual ferrofluid particles are around 150 $\mathrm{nm}$ in diameter and the typical width of a ferrofluid line is $1.6 \pm 0.2 \mu \mathrm{m}$ FWHM (full width at half maximum). Typical lengths of the ferrofluid lines are $16-50 \mu \mathrm{m}$. The detrimental effect on the quality of both the Hoechst fluorescence and bright-field images with increasing free ferrofluid concentrations is clearly illustrated in Figure 6.4. This is also the case for the $40 \mu \mathrm{g} / \mathrm{ml}$ of ferrofluid present with the current CellSearch system, Figure 6.4D.

\subsubsection{EFFECTS OF FREE FERROFLUID ON IMAGING OF CELLS FROM THE BREAST CANCER CELL LINE SKBR-3}

SKBR-3 cells were labeled with EpCAM-Ferrofluid and the nucleic acid dye Hoechst 33342 and sorted on lightscatter and Hoechst fluorescence to obtain ferrofluid labeled SKBR-3 cells devoid of free ferrofluids. Before placement in the sample chamber, free ferrofluid was added at different concentrations, ranging from 0 to $197 \mu \mathrm{g} / \mathrm{ml}$. The samples were placed in the CellTracks Magnest and scanned on the CellTracks TDI system for bright-field and Hoechst fluorescence. In Figure 6.5 the Hoechst fluorescence intensity and associated coefficient of variation are shown for the SKBR-3 cells. For 

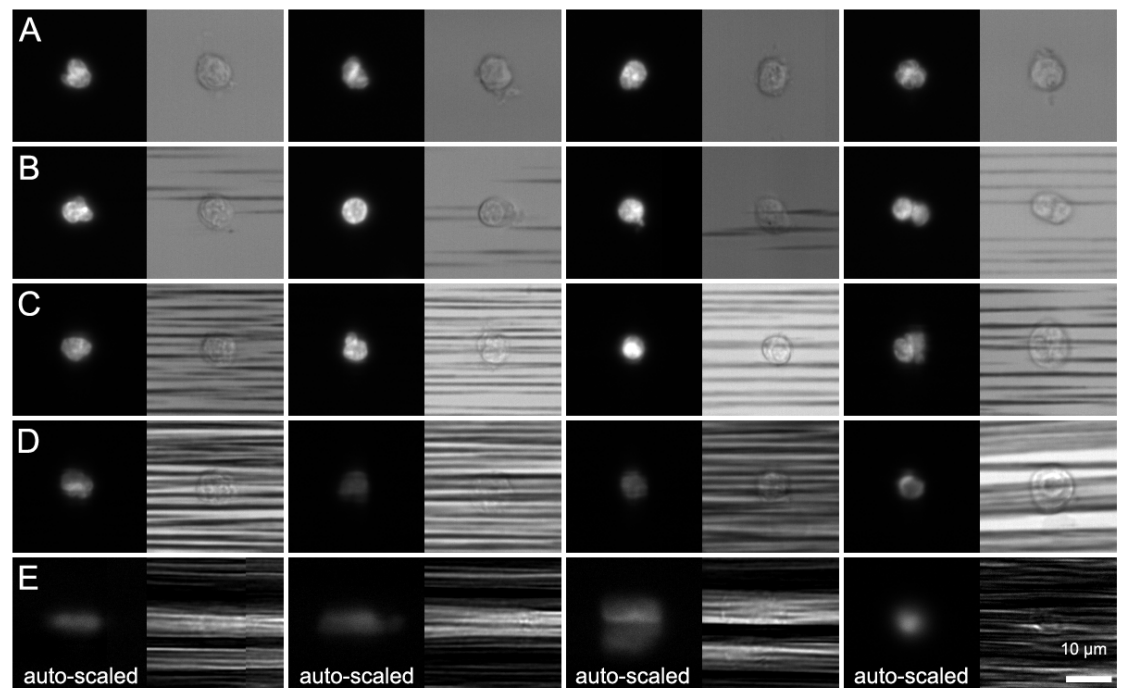

auto-scaled

auto-scaled

FIGURE 6.4 : Effect of free ferrofluid on Hoechst fluorescence and bright-field images of leukocytes. Panels A-E show free ferrofluid concentrations of: (A) $0 \mu \mathrm{g} / \mathrm{ml}$, (B) 2 $\mu \mathrm{g} / \mathrm{ml}$, (C) $10 \mu \mathrm{g} / \mathrm{ml}$, (D) $40 \mu \mathrm{g} / \mathrm{ml}$ and (E) $168 \mu \mathrm{g} / \mathrm{ml}$. In each Panel, 4 cells are shown with the Hoechst fluorescence image on the left and the bright-field image on the right. All bright-field images are scaled in intensity to the same lower and upper levels (range: 700-3000 DN) All fluorescence images, except in the last panel (E), are also scaled to the same lower and upper intensity (range: 0-2300 DN). Fluorescence images in panel E are auto-scaled as no fluorescence would be visible with the $0-2300 \mathrm{DN}$ setting.

analysis of the SKBR-3 cells, the total intensity was determined as the sum of all pixel intensities within a diameter of $20 \mu \mathrm{m}$ from the center of the cell.

The average total fluorescence intensity and its coefficient of variation remained constant up to $\sim 4 \mu \mathrm{g} / \mathrm{ml}$. For the concentration of $40 \mu \mathrm{g} / \mathrm{ml}$ the total intensity is $50 \%$ of the maximum value and the CV has risen to $41 \%$, compared to a sample without free ferrofluid. The CV of the total intensity of SKBR-3 nuclei without free EpCAM-Ferrofluid is $7.1 \%$ and rises to $7.5 \%$ for a concentration of $2 \mu \mathrm{g} / \mathrm{ml}$.

Figure 6.6 illustrates the effects of free ferrofluid on the fluorescence and bright-field images of SKBR-3 cells. These effects are similar to the effects that were observed for the T-lymphocytes (Figure 6.4). Free ferrofluid particles appear as stripes that interfere with bright-field image characterization with increasing ferrofluid concentrations. Also, parts of the fluorescence from the nuclei is blocked, reducing the accuracy of fluorescence image characterization. 


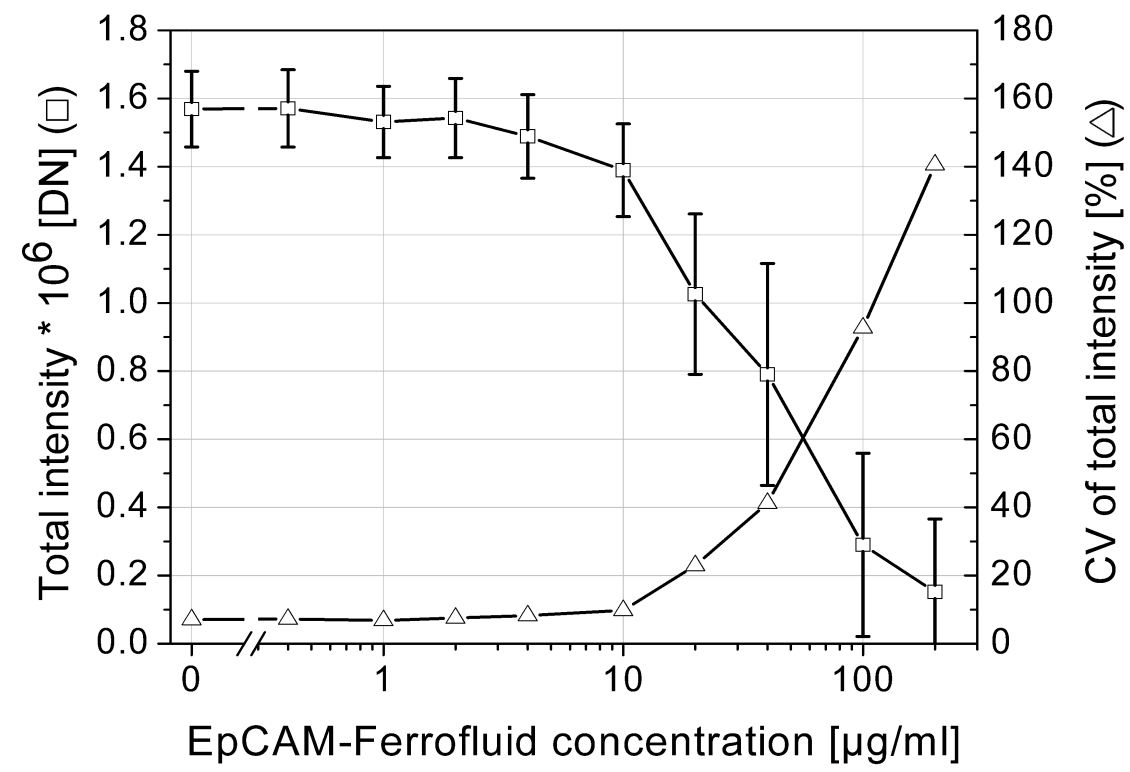

FigurE 6.5 : Effect of free EpCAM-Ferrofluid on the quantitative analysis of Hoechst 33342 staining of SKBR-3 cells. The average total fluorescence intensity (square markers) and associated coefficient of variation (CV) (triangular markers) are shown.

\subsubsection{COMPARISON OF EFFECTS ACROSS ALL OBJECT TYPES}

In the previous results sections we showed the quantitative effects of free ferrofluid on three bead types, T-lymphocytes and SKBR-3 cells. Table 6.2 combines the most important results to show the difference in effects of the free ferrofluid on these various object types. It is apparent that the effect of free ferrofluid is most profound on the T-lymphocytes. A 6-fold increase in $\mathrm{CV}$ of fluorescence intensity and a $67 \%$ decrease in fluorescence yield are observed at a concentration of $40 \mu \mathrm{g} / \mathrm{ml}$. The effects of free ferrofluid on the green and red beads are moderate. The last two columns in Table 6.2 show the results that will be obtained when the concentration of free ferrofluid in a sample is reduced to $2 \mu \mathrm{g} / \mathrm{ml}$. At such a concentration, the decrease in fluorescence yield and the increase in CV for the beads and SKBR-3 cells is small, while a moderate effect is still expected for the T-lymphocytes.

The table also shows that the effects on the blue beads, T-lymhpocytes and SKBR-3 cells are significantly different, although these objects emit fluorescence in the same wavelength range. For instance, the increase in $\mathrm{CV}$ and the reduction of the fluorescence intensity for the SKBR-3 cells are double that of the blue beads.

Figure 6.7 shows four bright-field images for blue beads (A), T-lymphocytes (B) and SKBR-3 cells (C). It shows that there is a clear difference in posi- 

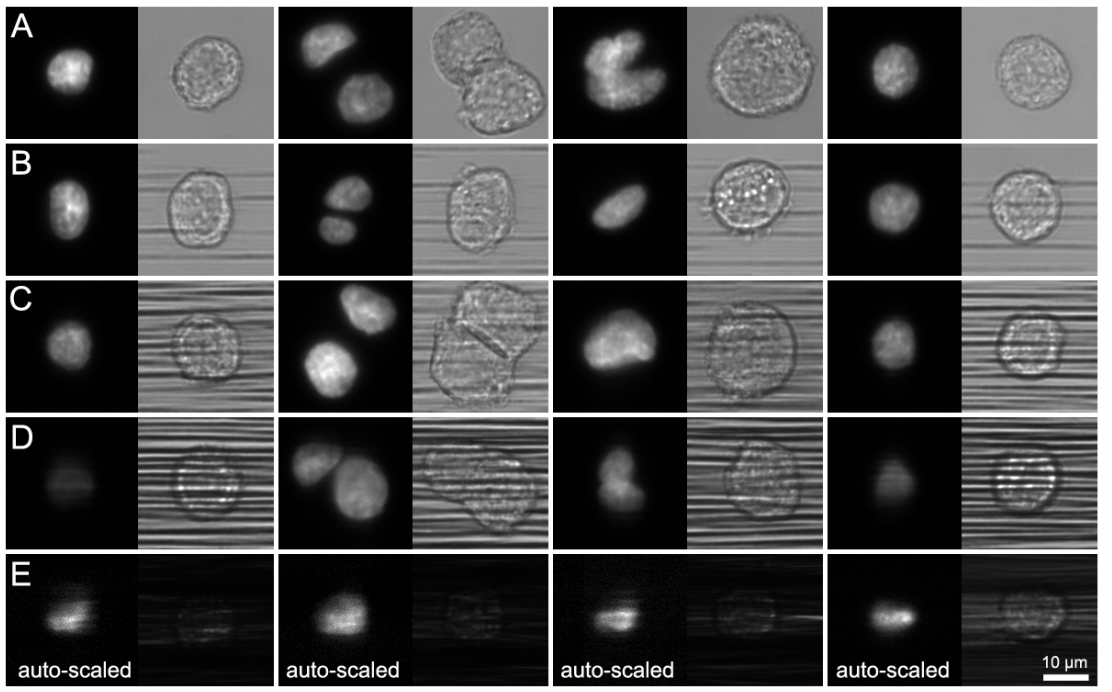

FIGURE 6.6 : Effects of free ferrofluid on Hoechst fluorescence and bright-field images of SKBR-3 cells. Panels A-E show free ferrofluid concentrations of: (A) $0 \mu \mathrm{g} / \mathrm{ml}$, (B) $2 \mu \mathrm{g} / \mathrm{ml}$, (C) $10 \mu \mathrm{g} / \mathrm{ml}$, (D) $40 \mu \mathrm{g} / \mathrm{ml}$ and (E) $200 \mu \mathrm{g} / \mathrm{ml}$. In each Panel, 4 cells are shown with the Hoechst fluorescence image on the left and the bright-field image on the right. All bright-field images are scaled in intensity to the same lower and upper levels (range: 200-2500 DN). All fluorescence images, except in the last panel (E), are also scaled to the same lower and upper intensity (range: 0-1400 DN). Fluorescence images in panel E are auto-scaled as no fluorescence would be visible with the 0-1400 DN setting.

TABLE 6.2 : Overview of quantitative effects of free ferrofluid on blue, green and red beads, T-Lymphocytes and SKBR-3 cells. The reduction in fluorescence is defined as the percentage decrease in fluorescence intensity as compared to a sample without free ferrofluid. The increase in $\mathrm{CV}$ is defined as the $\mathrm{CV}$ of the fluorescence intensity of the considered concentration divided by the $\mathrm{CV}$ of the fluorescence intensity of a sample without free ferrofluid.

\begin{tabular}{lllll}
\hline Object type & $\begin{array}{l}\text { Reduction in } \\
\text { fluorescence at } \\
40 \mu \mathrm{g} / \mathrm{ml}\end{array}$ & $\begin{array}{l}\text { Increase in CV } \\
\text { at } 40 \mu \mathrm{g} / \mathrm{ml}\end{array}$ & $\begin{array}{l}\text { Reduction in } \\
\text { fluorescence at } \\
2 \mu \mathrm{g} / \mathrm{ml}\end{array}$ & $\begin{array}{l}\text { Increase in CV } \\
\text { at } 2 \mu \mathrm{g} / \mathrm{ml}\end{array}$ \\
\hline Blue beads & $38 \%$ & $3.2 \times$ & $3 \%$ & $1.0 \times$ \\
Green beads & $12 \%$ & $1.5 \times$ & $3 \%$ & $0.9 \times$ \\
Red beads & $11 \%$ & $1.5 \times$ & $3 \%$ & $1.0 \times$ \\
\hline Lymphocytes & $67 \%$ & $6.0 \times$ & $16 \%$ & $1.4 \times$ \\
\hline SKBR-3 cells & $50 \%$ & $5.8 \times$ & $2 \%$ & $1.1 \times$ \\
\hline
\end{tabular}



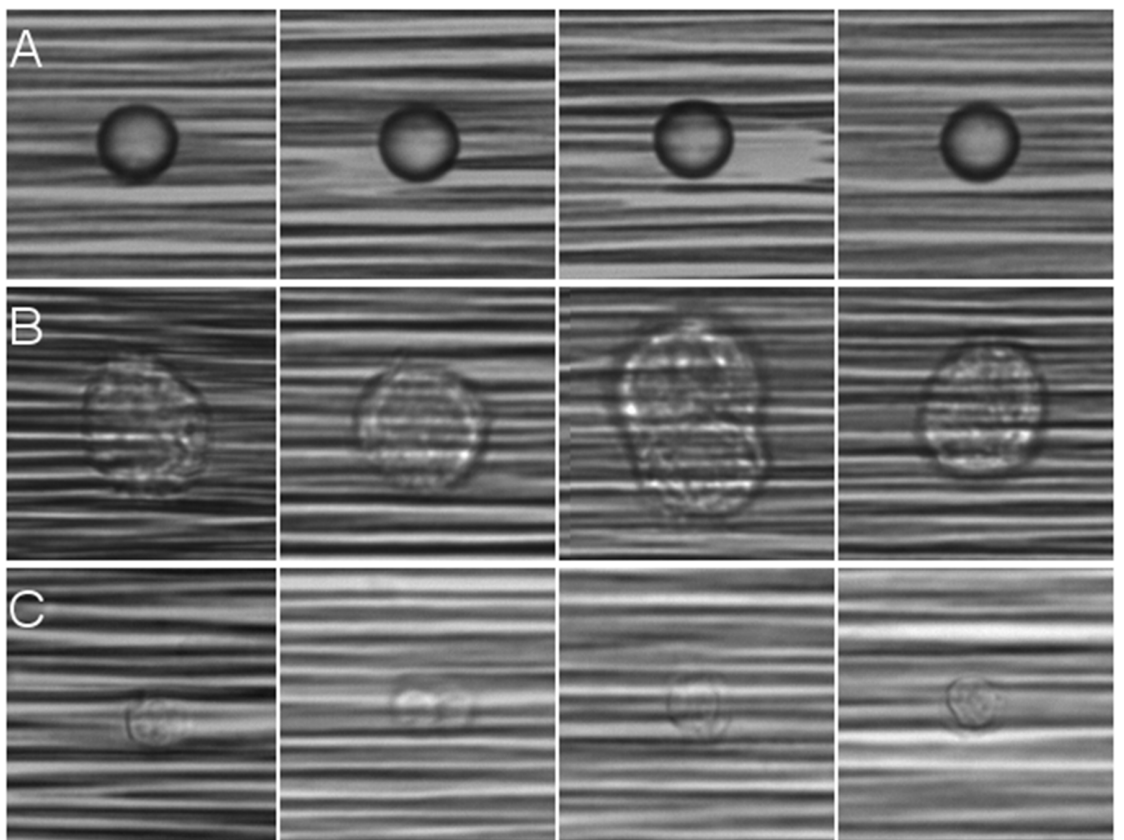

FIgURE 6.7 : Effect of free ferrofluid on position of beads and cells relative to the aggregated ferrofluid. The beads (A) appear to be located more above the ferrofluid, while the SKBR-3 cells (B) and T-Lymphocytes (C) are located below the ferrofluid.

tioning of the beads and cells at the imaging surface with respect to the aggregated ferrofluid. The beads appear to be located more above the ferrofluid, while the cells are covered by the ferrofluid.

\subsubsection{DETERMINATION OF THE CELL SURFACE MEMBRANE WITH A BRIGHT-FIELD IMAGE}

Figure 6.4 and 6.6 illustrated the difficulty of identifying the cell contours in the bright-field images with increasing free ferrofluid concentrations. The effect on the bright-field image signals with increasing concentrations of free ferrofluid is illustrated in Figure 6.8. The average background value and the corresponding standard deviation for each sample was determined by measurement of an area of $180 \times 180 \mu \mathrm{m}^{2}$ close to the center of the imaging surface, in which no cell was present. The first effect of increasing ferrofluid concentrations is observed by an increase in the coefficient of variation of the bright-field intensity followed by a decrease in the average bright-field intensity at concentrations higher than $4 \mu \mathrm{g} / \mathrm{ml}$. The average intensity for $40 \mu \mathrm{g} / \mathrm{ml}$, the standard sample concentration for samples that are prepared by the AutoPrep system, is around half of that of a sample without free 


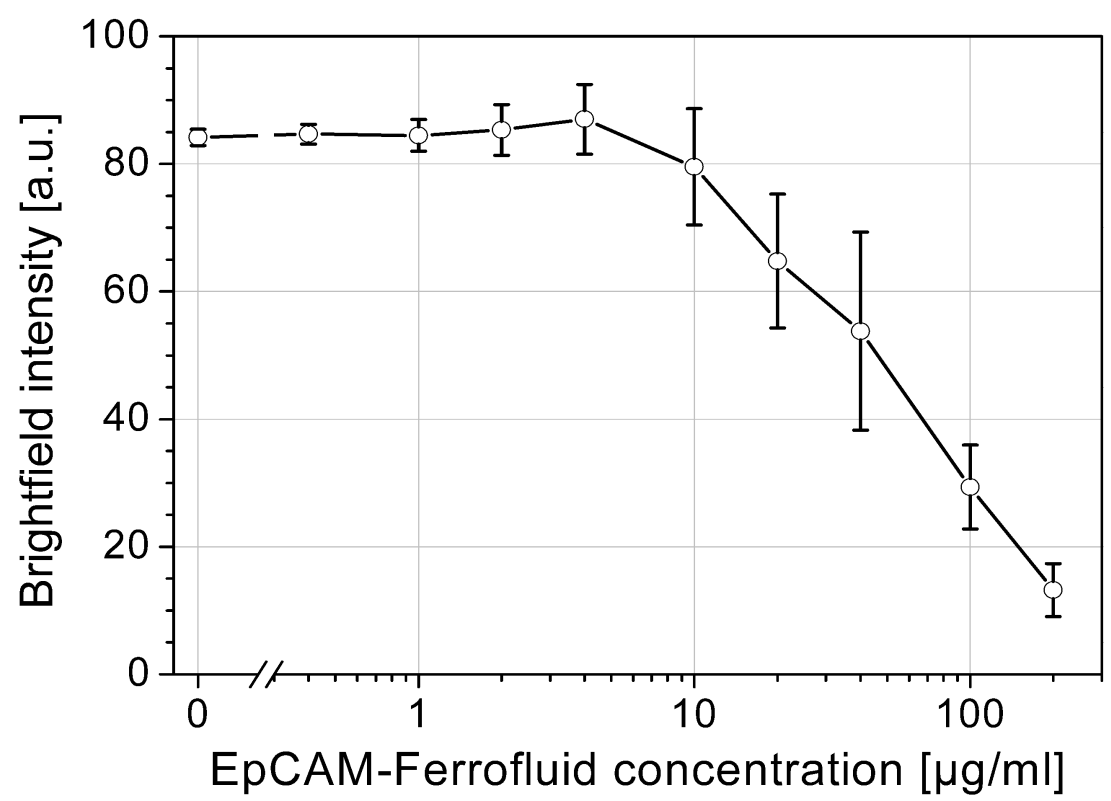

Figure 6.8 : Effect of free EpCAM-Ferrofluid on background bright-field intensity.

ferrofluid. The standard deviation is 12 -fold higher for a sample containing $40 \mu \mathrm{g} / \mathrm{ml}$ compared to a sample without free ferrofluid.

Identification of the contour of the cell or cell surface using bright-field images is achieved by first applying a Gaussian blur filter with a radius of 2 followed by determination of the edges using a Sobel edge detector. A median filter with a radius of 15 is then applied and the image is thresholded using the Li method [9]. Finally the holes in the thresholded image are filled and the resulting outline is shown in white on the original image as is illustrated in Figure 6.9 for SKBR-3 cells with $0 \mu \mathrm{g} / \mathrm{ml}(\mathrm{A}), 2 \mu \mathrm{g} / \mathrm{ml}$ (B) and $40 \mu \mathrm{g} / \mathrm{ml}(\mathrm{C})$ of free ferrofluid. The SKBR-3 cell in Panel A is correctly thresholded as indicated by the white line around the cell, while the other two suffer from contrast created in the image by the ferrofluid. In Panels D-F the same cells are shown only now a FFT filtering algorithm was applied to reduce the effect of the horizontal stripes of ferrofluid. This filter improved the detection of the cell surface for the cell shown in Panel E, but was not sufficient to identify the entire cell surface of the SKBR-3 cell with the higher free ferrofluid concentration shown in Panel F.

The method that was used to determine the outline of the cell in Figure 6.9E was further examined. SKBR-3 cells and T-lymphocytes from samples containing $2 \mu \mathrm{g} / \mathrm{ml}$ of free ferrofluid were used to determine its effectiveness. Figure 6.10 shows the result of the bright-field threshold algorithm for SKBR-3 cells (A) and T-lymphocytes (B) as a white outline. The outline 

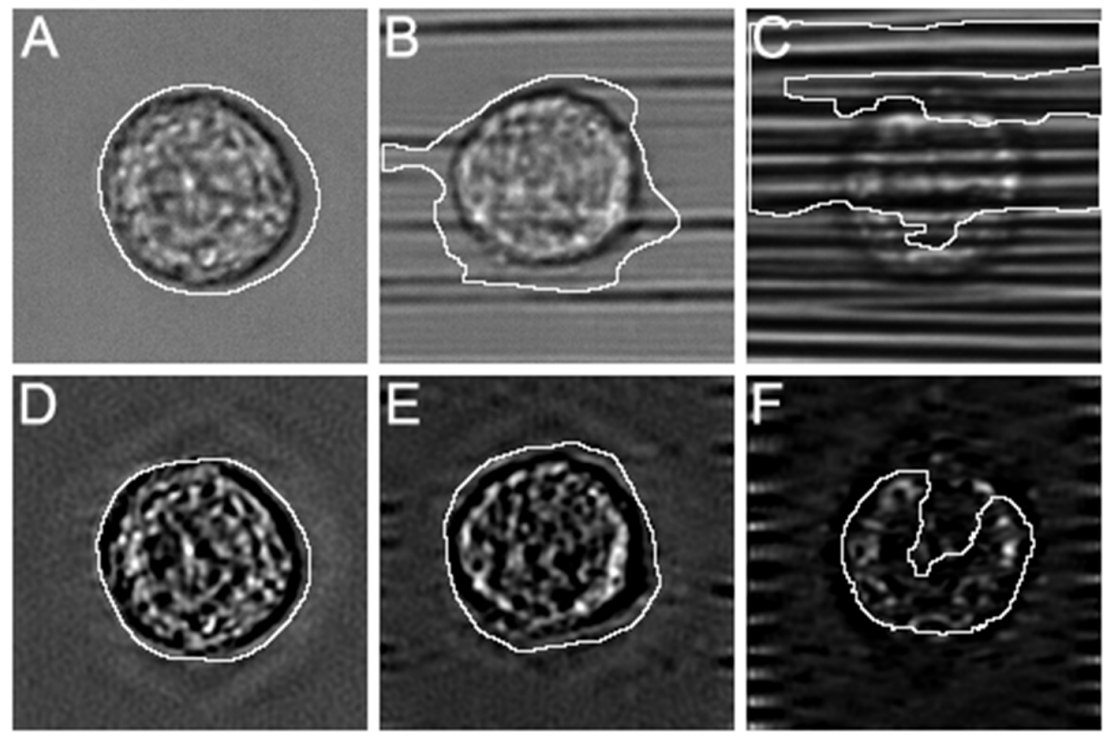

FiguRE 6.9 : Effect of free ferrofluid on the identification of the cell surface of SKBR-3 cells using bright-field. Concentrations of free ferrofluid are $0 \mu \mathrm{g} / \mathrm{ml}$ (A and D), 2 $\mu \mathrm{g} / \mathrm{ml}$ (B and $\mathbf{E}$ ) and $40 \mu \mathrm{g} / \mathrm{ml}(\mathbf{C}$ and $\mathbf{F})$. The white line indicates the outline of the cell that was detected using the original image (top row) and an FFT filtered image (bottom row).

as determined by an Otsu threshold algorithm on the nuclear fluorescence images is shown as a blue outline. The contours of the SKBR-3 cells as well as the T-lymphocytes are correctly determined using the bright-field threshold method.

\subsection{Discussion}

The presence of CTC is associated with poor outcome in metastatic cancer patients [10-13]. In most cases the frequency of CTC is extremely low (1-10/7.5 ml of blood) making accurate detection of utmost importance. CTC can also be used to assess the expression of treatment targets and for this application accurate and sensitive techniques are required. In the CellSearch system, CTC are immunomagnetically enriched from $7.5 \mathrm{ml}$ of blood. The enriched samples of $370 \mu \mathrm{l}$ contain 873-5245 leukocytes [14], CTC expressing EpCAM and $40 \mu \mathrm{g} / \mathrm{ml}$ of free ferrofluid. This free ferrofluid absorbs and scatters part of the fluorescence that is emitted by the cells and disturbs bright-field imaging.

In this study we investigate the influence of free ferrofluid on the ability to accurately detect and characterize CTC. Increasing amounts of free 

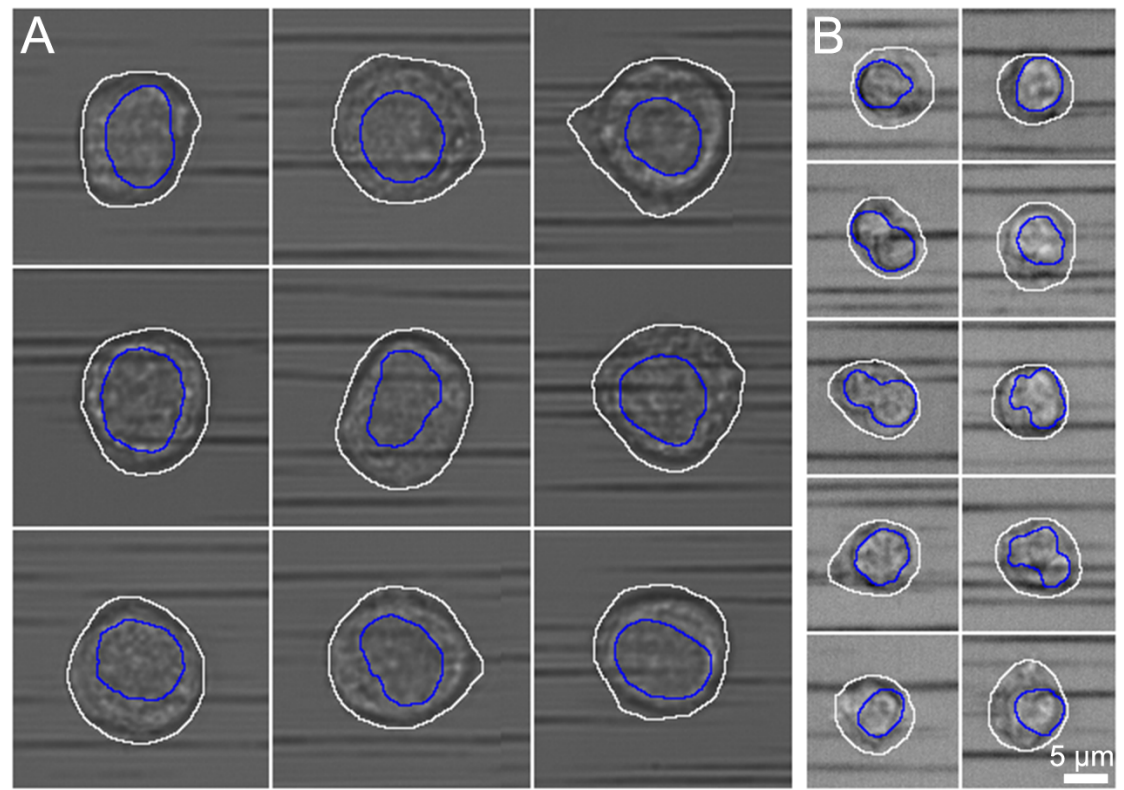

Figure 6.10 : Bright-field images of SKBR-3 cells (A) and T-lymphocytes (B) from a sample containing $2 \mu \mathrm{g} / \mathrm{ml}$ of free ferrofluid. The white outline around each cell indicates the border of the event that was detected using bright-field thresholding. The blue outline inside the cell indicates the border of the nucleus as determined by an Otsu threshold of the respective Hoechst fluorescence images. The scale in both panels is identical and the images in panel B were cropped to accommodate for the smaller size of the lymphocytes.

ferrofluid were added to magnetically and fluorescently labeled beads, Tlymphocytes and tumor cells from the breast cancer cell line SKBR-3 to assess the influence on the fluorescence measurements. A reduction of the fluorescence signals started to become apparent at concentrations higher than $2 \mu \mathrm{g} / \mathrm{ml}$. At a concentration of $40 \mu \mathrm{g} / \mathrm{ml}, 11-67 \%$ of the fluorescence signals were lost and the CV of the fluorescence signals from cells increased from 7 to $40 \%$. A significant difference in effect of free ferrofluid on blue beads and blue fluorescing T-lymphocytes and SKBR-3 cells was observed. This is most likely due to the difference in travel times for these objects from the sample solution to the top of the CellSearch cartridge under the influence of the magnetic field. The magnetic moment of immunomagnetically labeled cells is significantly lower than that of magnetic beads. This then results in different configurations at the analysis surface; the beads are positioned more above the ferrofluid, while the leukocytes take longer to get to the surface and are therefore more below the ferrofluid.

Correct identification of the cell surface is important for the extraction of morphological features as well as quantification and distribution of antigens on the cell surface, cytoplasm and nucleus of a cell. The threshold used 
to identify events that are potentially CTC in the CellSearch system is set on the expression of Cytokeratin-PE. The distribution of the cytokeratin staining can however vary greatly in CTC especially those undergoing apoptosis $[15,16]$. The use of intracellular or cell surface antigens recognized by fluorescently labeled antibodies to identify the cytoplasmic membrane could be hampered by the inhomogeneous distribution of the antigens or the low expression levels of these antigens. Specific labeling of the cytoplasmic membrane followed by its recognition could be an option, but will reduce the number of fluorescent probes available to detect antigens of interest. The use of nuclear staining to correctly identify the cell surface is not sufficient as the size of the cytoplasm of cells relative to its nucleus can vary greatly. Availability of a bright-field image of the cell in which the cytoplasmic membrane can clearly be identified can solve this problem. Free ferrofluid however prevents the use of bright-field for the identification of the cytoplasmic membrane, as illustrated in Figure 6.9. For detection of CTC this results in the inability to detect those CTC with a relatively low antigen density and reduces the ability to detect the presence of treatment targets with the same sensitivity across the detected CTC. In addition the cell surface cannot be accurately determined which decreases the ability to determine the surface of which treatment targets need to be examined. Reduction of free ferrofluid below $2 \mu \mathrm{g} / \mathrm{ml}$ is needed to enable the use of the bright-field images of the cells to identify their cytoplasmic membrane. 


\subsection{ReFERENCES}

[1] M. Kagan, D. Howard, T. Bendele, J. Mayes, J. Silvia, M. Repollet, J. Doyle, J. Allard, N. Tu, T. Bui, T. Russell, C. Rao, M. Hermann, H. Rutner, and L. Terstappen, "A sample preparation and analysis system for identification of circulating tumor cells," J Clinical Ligand Assay, vol. 25(1), pp. 104-110, 2002.

[2] A. Tibbe, B. de Grooth, J. Greve, P. Liberti, G. Dolan, and L. Terstappen, "Optical tracking and detection of immunomagnetically selected and aligned cells," Nature Biotechnology, vol. 17, pp. 1210-1213, 1999.

[3] A. Tibbe, B. de Grooth, J. Greve, G. Dolan, C. Rao, and L. Terstappen, "Magnetic field design for selecting and aligning immunomagnetic labeled cells," Cytometry, vol. $47(3)$, pp. 163-172, 2002.

[4] T. Scholtens, F. Schreuder, S. Ligthart, J. Swennenhuis, A. Tibbe, J. Greve, and L. Terstappen, "Celltracks TDI: An image cytometer for cell characterization," Cytometry Part A, vol. 79(3), pp. 203-213, 2011.

[5] F. Schreuder, Laser image cytometer for analysis of circulating tumor cells. PhD thesis, PhD Thesis University of Twente, ISBN 9789036526104, 2008.

[6] W. Rasband, "Imagej," http://rsbweb.nih.gov/ij/index.html, vol. -, pp. -, 1997 2010.

[7] N. Otsu, "Threshold selection method from gray-level histograms," IEEE Trans Syst Man Cybern, vol. SMC-9(1), pp. 62-66, 1979.

[8] P. Liao, T. Chen, and P. Chung, "A fast algorithm for multilevel thresholding," Journal of Information Science and Engineering, vol. 17(5), pp. 713-727, 2001.

[9] C. Li and P. Tam, "An iterative algorithm for minimum cross entropy thresholding," Pattern Recognition Letters, vol. 18(8), pp. 771-776, 1998.

[10] L. Terstappen, C. Rao, S. Gross, and A. Weiss, "Peripheral blood tumor cell load reflects the clinical activity of the disease in patients with carcinoma of the breast," Int J Onc, vol. 17(3), pp. 573-578, 2000.

[11] M. Cristofanilli, T. Budd, M. Ellis, A. Stopeck, J. Matera, M. Miller, J. Reuben, G. Doyle, W. Allard, L. Terstappen, and D. Hayes, "Circulating tumor cells, disease progression, and survival in metastatic breast cancer," N Engl J Med, vol. 351(8), pp. 781-791, 2004.

[12] S. Cohen, C. Punt, N. Iannotti, B. Saidman, K. Sabbath, N. Gabrail, J. Picus, M. Morse, E. Mitchell, C. Desch, M. Miller, G. Doyle, H. Tissing, L. Terstappen, and N. Meropol, "The relationship of circulating tumor cells to tumor response, progression-free survival, and overall survival in patients with metastatic colorectal cancer," Journal of Clinical Oncology, vol. 26(19), pp. 3213-3221, 2008.

[13] J. De Bono, H. Scher, R. Montgomery, C. Parker, M. Miller, H. Tissing, G. Doyle, L. Terstappen, K. Pienta, and D. Raghavan, "Circulating tumor cells predict survival benefit from treatment in metastatic castration resistant prostate cancer," Clin Can Res, vol. 14(19), pp. 6302-6309, 2008.

[14] T. Scholtens, F. Schreuder, S. Ligthart, J. Swennenhuis, A. Tibbe, J. Greve, and L. Terstappen, "Automated identification of circulating tumor cells by image cytometry," Cytometry Part A, vol. 81(2), pp. 138-148, 2012.

[15] C. Larson, J. Moreno, K. Pienta, S. Gross, M. Repollet, S. OHara, T. Russell, and L. Terstappen, "Apoptosis of circulating tumor cells in prostate cancer patients," Cytometry part A, vol. 62(1), pp. 46-53, 2004. 
[16] F. Coumans, C. Doggen, G. Attard, J. de Bono, and L. Terstappen, "All circulating $\mathrm{EpCAM}+\mathrm{CD} 45-\mathrm{CK}+$ but not EpCAM $+\mathrm{CD} 45+\mathrm{CK}+$ objects predict overall survival in castration-resistant prostate cancer," Annals of Oncology, vol. 21(9), pp. 18511857, 2010. 



\section{CHAPTER}

\section{REMOVAL OF FREE FERROFLUID AFTER IMMUNOMAGNETIC ENRICHMENT OF CTC}

In the CellSearch system, EpCAM-Ferrofluid is used to immunomagnetically enrich Circulating Tumor Cells (CTC) from $7.5 \mathrm{ml}$ of blood. Free ferrofluid remains in the sample after processing and interferes with CTC detection. Removal of free ferrofluid will increase the fluorescence yield, lower the CV and enable the use of the bright-field images from a detected event, thereby increasing the specificity of CTC detection and improving the ability to detect the presence of treatment targets.

A setup was constructed that rotates a sample tube from the AutoPrep instrument around its longitudinal axis and aspirates fluid from the rotating tube, thereby removing free ferrofluid while keeping CTC loss to a minimum. This process was simulated in Matlab, optimized using buffer or blood spiked with SKBR-3 and or PC3-9 cells and tested in blood samples from cancer patients.

Parameters used for removal of free ferrofluid were determined from simulations and spiking experiments. Using 6 ferrofluid withdrawal steps with a rotation period of $20 \mathrm{~min}, 96.1 \pm 1.3 \%$ (median $\pm \mathrm{SD}$ ) of free ferrofluid was removed with a median recovery of $97.8 \pm 3.2 \%$ of spiked tumor cells in buffer. Blood samples from healthy donors spiked with SKBR-3 and or PC3-9 cells showed a recovery between $68.2 \%$ and $84.3 \%$ (median $76.6 \%$, SD 29.7\%) and a median ferrofluid removal efficiency of $94.7 \pm 1.8 \%$. Blood samples from 11 prostate cancer patients showed a 
median CTC recovery of $55.8 \pm 33.3 \%$, with a median ferrofluid removal efficiency of $94.2 \pm 2.2 \%$.

$>95 \%$ of free ferrofluid can be removed by centrifugation while maintaining $\sim 77 \%$ of tumor cells spiked into $7.5 \mathrm{ml}$ of blood. Although similar removal of free ferrofluid could be obtained with patient samples, the recovery of CTC was less. To determine whether or not improvement using the centrifugal approach is feasible the actual density of CTC will need to be established and compared to that of leukocytes carried over from the immunomagnetic enrichment.

\subsection{INTRODUCTION}

Presence of Circulating Tumor Cells (CTC) in metastatic carcinoma patients is associated with poor overall and progression free survival [1-3]. These CTC are usually present in low concentrations and a threshold of 5 CTC per $7.5 \mathrm{ml}$ of blood is used to differentiate between patients with favorable and unfavorable prognosis $[1,4]$. The $7.5 \mathrm{ml}$ of blood consists for $\sim 45 \%$ of cells, the majority of the cells are red blood cells $\left(\sim 3 \times 10^{10}\right)$, followed by platelets $\left(\sim 2 \times 10^{9}\right)$ and white blood cells $\left(\sim 5 \times 10^{7}\right)$. A highly sensitive and specific method is clearly needed to detect the threshold number of 5 CTC in $7.5 \mathrm{ml}$ of blood.

To increase the relative concentration of CTC in the blood collected from a patient, we use the CellTracks Autoprep system. In this system CTC enrichment is achieved by immunomagnetic labeling of CTC using EpCAM labeled ferrofluid. After enrichment a sample of $360 \mu \mathrm{l}$ is obtained containing 873-5245 (mean 1989) white blood cells and $14.4 \mu \mathrm{g}$ of free ferrofluid. This excess of free ferrofluid inhibits the fluorescence signals. For example the fluorescence yield of SKBR-3 cells that are labeled with Cytokeratin-PE is reduced by $50 \%$ and the $\mathrm{CV}$ of the measured total fluorescence intensity increased by 5.8 fold. Reduction of the free ferrofluid concentration from $40 \mu \mathrm{g} / \mathrm{ml}$ to $2 \mu \mathrm{g} / \mathrm{ml}$ practically eliminated the detrimental influence on fluorescence- and bright-field imaging (Chapter 6). This chapter describes a centrifugal method to remove most of the excess ferrofluid from CTC samples, while keeping CTC loss to a minimum.

\subsection{Materials And Methods}

\subsubsection{SKBR-3 CELLS IN BUFFER}

Samples for the initial testing of the automated ferrofluid removal setup (AFRS) consisted of cells from the breast cancer cell line SKBR-3 spiked in dilution buffer (Veridex, Raritan, NJ, USA). The cells were magnetically and fluorescently labeled with reagents from the CellSearch CTC kit (Veridex). The volume of each single sample was equal to the CellTracks AutoPrep final volume of $360 \mu \mathrm{l}$. Samples were prepared in duplicate volumes of 
$720 \mu$ to minimize variations in the number of spiked cells. To each sample, $60 \mu \mathrm{l}$ EpCAM-FerroFluid (EpCAM-FF) $(12 \mu \mathrm{g}), 20 \mu \mathrm{l}$ DAPI, 20 $\mu \mathrm{l}$ staining reagent (CK-PE and CD45-APC) and $20 \mu \mathrm{l}$ permeabilization reagent were added to $600 \mu \mathrm{l} \mathrm{SKBR-3}$ cells (at $\sim 10$ cells $/ \mu \mathrm{l}$ ). The samples were incubated in the dark at room temperature for 20 minutes. After preparation of the duplicate sample, one half was used as a control sample, which was not processed for ferrofluid removal, and the other half was processed for ferrofluid removal.

\subsubsection{SKBR-3 AND PC3-9 CELLS SPIKED IN WHOLE BLOOD SAMPLES FROM HEALTHY DONORS}

Venous blood was drawn in $10 \mathrm{ml}$ CellSave preservative tubes from 11 healthy volunteers. An even number of tubes, up to a maximum of 8, were drawn from each volunteer and all tubes were numbered sequentially. Blood samples were processed within 72 hours from blood draw using the CellTracks AutoPrep system (Veridex). Cells from the prostate cancer cell line PC3-9 with relatively low EpCAM antigen density ( 50.000 molecules per cell) and SKBR-3 cells with relatively high EpCAM antigen density ( $\sim 500.000$ molecules per cell) and expressing her2/neu were used for spiking experiments [5]. EpCAM expression density of PC3-9 cells is within the range detected on CTC from cancer patients [5]. Aliquots of $7.5 \mathrm{ml}$ of blood from each CellSave tube were spiked with $110 \mu \mathrm{l}$ of both SKBR-3 and PC3-9 cells for a total of 1000 spiked cells for each cell type. These samples were then centrifuged at $800 \mathrm{~g}$ for 10 minutes and placed in the CellTracks AutoPrep system. The standard CTC protocol was followed except for the final transfer of the sample to the analysis cartridge and the addition of anti-her2-FITC to distinguish between PC3-9 and SKBR-3 cells.

\subsubsection{BLOOD SAMPLES FROM CARCINOMA PATIENTS}

Duplicate blood samples were collected from 11 carcinoma patients that were enrolled in phase I/II Abiraterone trial studies [6] conducted at the Royal Marsden Hospital in London, UK. Venous blood was drawn in 10 $\mathrm{ml}$ CellSave preservative tubes and shipped at room temperature the same day to the Veridex laboratory in the Netherlands (Veridex LLC, Enschede, The Netherlands). The blood samples were processed within 72 hours from blood draw using the CellTracks AutoPrep system and the adapted CTC protocol as described above. The conical tubes containing the processed samples were removed from the AutoPrep, capped and transferred to the site at which they would be processed for removal of free ferrofluid.

\subsubsection{MEASUREMENT OF FREE FERROFLUID CONCENTRATION}

The concentration of free ferrofluid in a sample was determined by absorbance measurements on a UV-2401 PC spectrophotometer (Shimadzu, 
Den Bosch, Netherlands). Ten samples were prepared with decreasing concentrations of EpCAM-Ferrofluid (EpCAM-FF) to calibrate the absorbance measurements. The cuvette used has a path length of $10 \mathrm{~mm}$ and a volume of $150 \mu \mathrm{l}$. The sample with the highest concentration was prepared by adding $75 \mu \mathrm{l}$ of EpCAM-FF at $200 \mu \mathrm{g} / \mathrm{ml}$ to $75 \mu \mathrm{l}$ of de-ionized water (DI Water). After measuring the absorbance spectrum, the next sample was prepared by first removing $75 \mu \mathrm{l}$ from the first sample after mixing, followed by addition of $75 \mu \mathrm{l}$ of DI water. The absorbance spectrum of this 2 -fold diluted sample was determined and 2 -fold dilution steps were repeated until 10 samples (9 dilutions) were measured. Furthermore, a baseline using only DI water was run before the first sample to correct the measurements of the samples containing EpCAM-Ferrofluid. This process was repeated twice for a total of 3 absorbance measurements for each concentration. After analysis of the spectra the optimal value for the absorbance measurements was determined to be $420 \mathrm{~nm}$. This wavelength allows for detection of a broad range of concentrations from 0.1 to $161 \mu \mathrm{g} / \mathrm{ml}$. The obtained calibration graph is shown in Figure 7.1. The calibration data were used to determine the concentration of EpCAM-Ferrofluid in samples used in optimization and testing of the AFRS.

\subsubsection{CELLTRACKS AUTOPREP}

The CellTracks AutoPrep [7, 8] is a system for automated immunomagnetic enrichment and fluorescent labeling of tumor cells in blood samples from cancer patients. Eight blood samples can be processed simultaneously. First, $7.5 \mathrm{ml}$ of blood from a CellSearch Preservative Tube (Veridex) is transferred into a $15 \mathrm{ml}$ conical tube, centrifuged for 10 minutes at 800 $\mathrm{g}$ and then placed in the CellTracks AutoPrep. The system consists of nine stations, each performing a specific task. In short, EpCAM labeled antibodies bound to ferrofluids are used to immunomagnetically label cells of epithelial origin. The magnetically enriched cells are fluorescently labeled with the nucleic acid dye DAPI, Phycoerythrin-labeled antibodies directed against Cytokeratins 8, 18 and 19 (CK-PE), and Allophycocyanin-labeled antibodies against CD45 (CD45-APC).

In this study the CellTracks AutoPrep was used to process blood samples from cancer patients and blood samples from healthy donors that were spiked with SKBR-3 and PC3-9 cells. The default CTC labeling protocol from the CellSearch kit was used except for the last step of the process, which transfers the sample to an analysis cartridge.

The standard CTC protocol that is used in the AutoPrep to process CTC samples deposits the final sample in a CellTracks Cartridge (Cell Presentation device), which is placed inside a CellTracks Magnest ${ }^{\mathrm{TM}}$. This helps to bring the magnetically labeled cells to the analysis surface in preparation for imaging. To prevent the unnecessary step of removing the sample from the cartridge in a later stage, the finished sample, around 360 


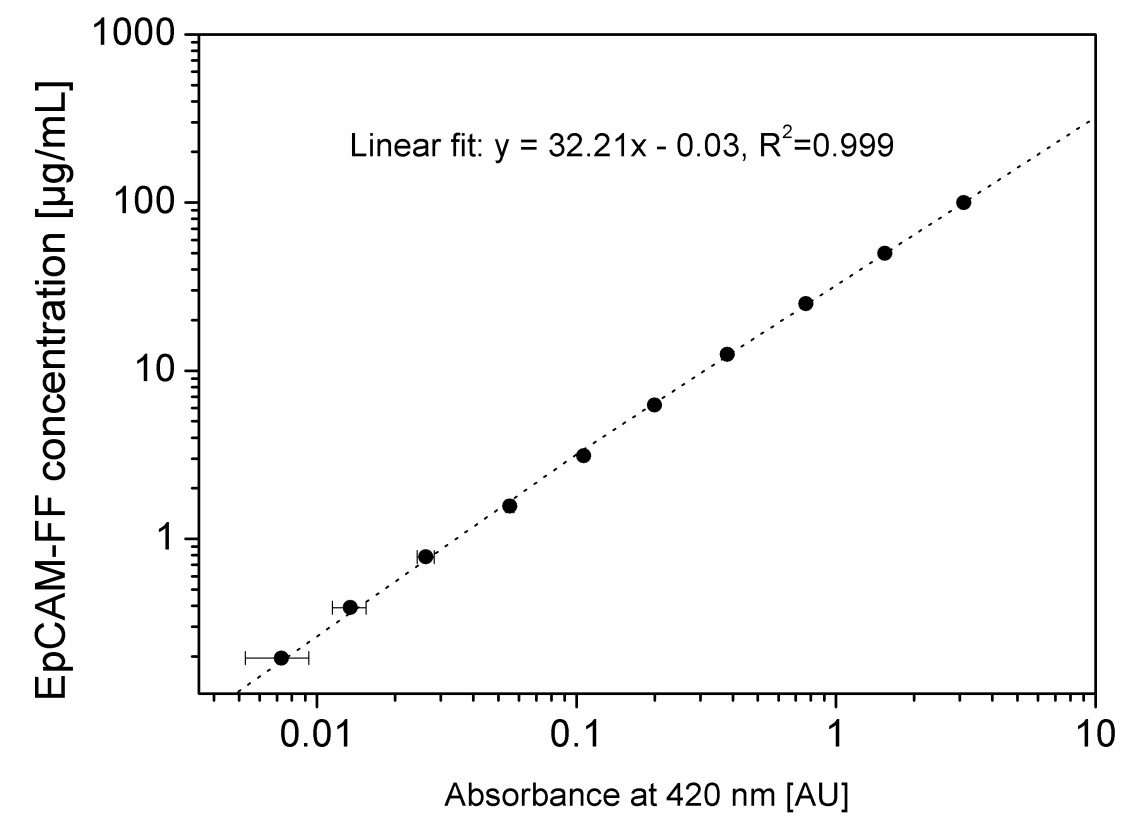

FIgURE 7.1 : Calibration graph of EpCAM-Ferrofluid concentration versus absorbance at $420 \mathrm{~nm}$. The maximum absorbance value that can be measured on the spectrophotometer is 5, giving a maximum detectable concentration of EpCAM-Ferrofluid of 161 $\mu \mathrm{g} / \mathrm{ml}$. Data points show the standard deviation from the 3 absorbance measurements at each concentration. Dotted line indicates the linear fit.

$\mu \mathrm{l}$, was left in the conical tube. The tubes containing the processed samples were removed from the CellTracks AutoPrep system, capped and transferred to the site at which they would be further processed for ferrofluid removal.

\subsubsection{AUTOMATED FERROFLUID REMOVAL SETUP (AFRS)}

The AFRS was constructed to remove free ferrofluid from samples containing CTC and with the possibility of integration into the sample preparation procedure of the CellTracks AutoPrep system. The setup was designed to remove $\geq 95 \%$ of the free ferrofluid in a sample while removing less than $10 \%$ of relevant cells (CTC) in the process. It rotates a conical tube, containing a fluidic sample, usually $360 \mu$, of cells and ferrofluid, around its longitudinal axis, forcing the cells to the outside of the tube. When the cells reach the wall of the tube they are forced up due to the conical shape of the tube. The ferrofluids, being $~ 70$ times smaller in diameter, are not significantly affected by the centrifugal force and stay approximately randomly distributed. This leads to a difference in distribution of the cells and ferrofluids over time, as the tube is being rotated, which is exploited by 
removing a fraction of the fluidic sample from the bottom of the rotating tube after a set period of time. Since most of the cells are now located near the top of the sample, the removed fraction contains mostly ferrofluids. After removal of the fluidic sample, the same amount of System Buffer (Veridex, Raritan, NJ, USA) is added to the rotating tube. This process is repeated up to five times, resulting in the repeated removal of a fixed percentage of the ferrofluids, without significant removal of cells of interest. Figure 7.2 shows a photograph of the AFRS to illustrate the components that were used in the setup.

The AFRS combines several components to enable the controlled and precise removal of sample fluid from a rotating tube, shown in Figure 7.3. A custom holder was designed and build, (part $\mathrm{H}$ in Figure 7.2), to accommodate a conical tube from the AutoPrep system, which is held in place by gently pushing it down into the holder. The bottom of the holder was designed to center the tube, preventing off-axis rotation. Also, the top ring of the tube-holder is slightly narrower than the outer diameter of the tube to firmly hold it in place during rotation. Another holder was fabricated to accommodate the tube-holder and motor underneath, two 15 ml tubes (Greiner Bio-one, Alphen a/d Rijn, Netherlands) that contain buffer and waste fluids, and a Magnest with cartridge to collect samples of interest. Also, during testing of the setup, fractions of the sample that were removed were collected here to check for free ferrofluid removal- and CTC recovery efficiency. The motor rotating the conical tube is a 5-phase stepper motor (Vexta PK543AUA, Oriental-motor Corp., Braintree, MA, USA) controlled by a TTL signal generated by a DAQ card in an external computer, controlled by Labview. The TTL signal is directed to a Nanostep controller (Vexta DFR1507A, Oriental-motor Corp). The Labview program is used to generate a controlled signal to ramp the motor rpm from 0 to its final rpm in 15 seconds, preventing turbulence. Since higher rotational speeds will result in higher recovery of cells in this method, we used the maximum practical rotational speed of the 5-phase stepper motor that still resulted in stable rotation of the conical tube. This speed was determined to be $900 \mathrm{rpm}$. The probe arm, indicated by part $\mathrm{E}$ in Figure 7.2, is used to move two pipettes, indicated by part $\mathrm{F}$, in the horizontal and vertical direction. This assembly is similar to the ninth station in the CellTracks AutoPrep system. The horizontal movement, direction left to right in the picture, of the probe arm was calibrated to position either probe above the center of the rotating tube, buffer or waste bottle or the sample cartridge. The vertical movement of the pipettes connected to the probe arm was calibrated to position them at the bottom of the rotating conical tube, without touching the bottom, and near the bottom of the buffer and waste bottles. Also, the vertical position and movement of the rightmost pipette, while inside the sample cartridge, was calibrated to move up as the sample is deposited inside the cartridge to prevent the formation of air bubbles. Calibration of the various probe positions was repeated on a bi-weekly basis. 


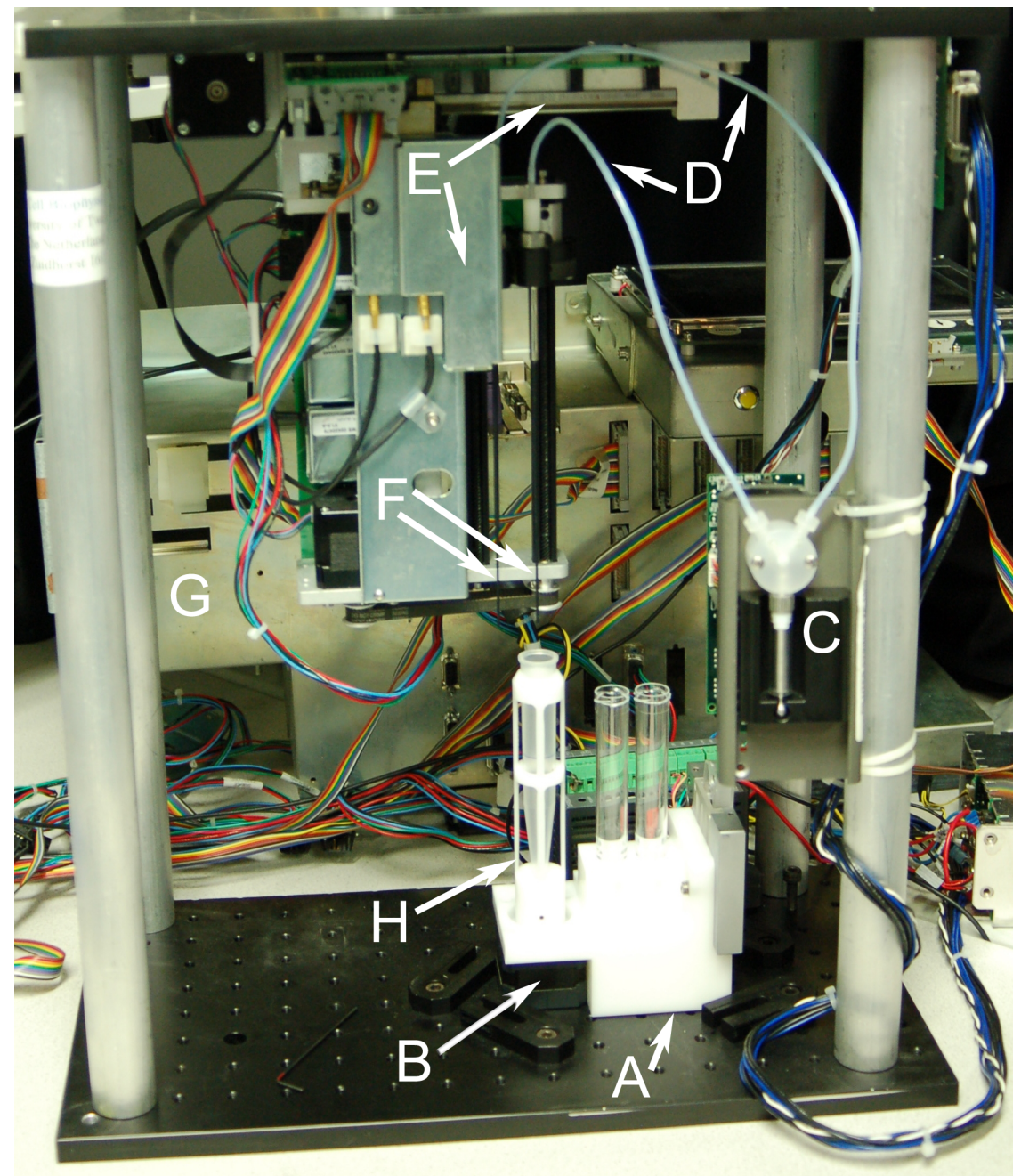

FigURE 7.2 : Photograph showing an overview of the automated ferrofluid removal setup. (A) Custom manufactured holder, shown in white, to accommodate motor and rotating conical tube on the left, two $15 \mathrm{ml}$ containers for buffer and waste in the middle, and a Magnest Cell Presentation Device to the right. (B) Five-phase stepper motor (underneath white holder) used to rotate the conical tube-holder. (C) Syringe pump, working volume $1 \mathrm{ml}$, with switchable entry and exit ports connected to (D) two polyethylene fluidic lines (Inner diameter (ID) $1.69 \mathrm{~mm}$ / Outer diameter (OD) 2.54 $\mathrm{mm}$ ). (E) Pipetting arm with guide rail and (F) two pipettes (ID $0.27 \mathrm{~mm} / \mathrm{OD} 0.57$ $\mathrm{mm}$ ). (G) Control computer, for pipetting station and syringe pump, running on Linux and custom software (Veridex). (H) Custom manufactured holder to accommodate a conical tube from the CellTracks AutoPrep. 

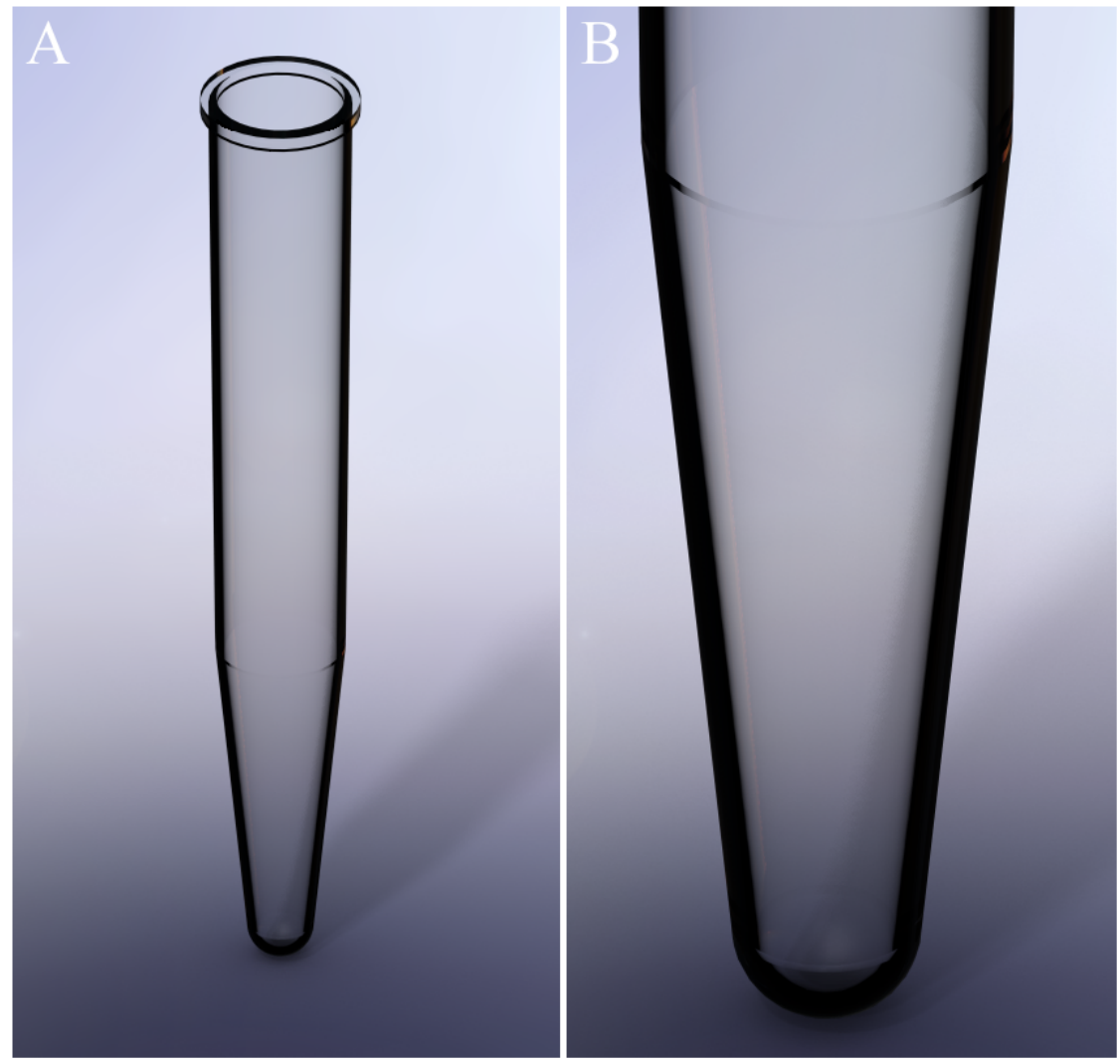

Figure 7.3 : (A) Overview drawing of conical tube. It consists of two parts: an almost cylindrical upper part and a conical shaped lower part, which has a slope of $6^{\circ}$. (B) Close-up view of the lower part of the conical tube. The length of the entire tube is 120 $\mathrm{mm}$ and the inner diameter varies from $15 \mathrm{~mm}$ at the top to $5.5 \mathrm{~mm}$ at the bottom of the tube. The thickness of the tube wall is $1 \mathrm{~mm}$. A $360 \mu \mathrm{l}$ sample, as used in most experiments, fills the tube to a height of $11.6 \mathrm{~mm}$ above the bottom. This amounts to $25 \%$ of the height of the conical section of the tube. 
A syringe pump, shown in part $\mathrm{C}$ in Figure 7.2, was used to aspirate and deposit fluidic samples from both pipettes using a switchable entry and exit port connected by polyethylene tubing. The syringe pump was used to prime the entire fluidic system with System Buffer before use to remove air bubbles. Also, it was used to aspirate a fraction of the sample in the rotating tube and replace it with the same volume of System Buffer. We used the slowest aspiration speed setting possible $(16.7 \mu \mathrm{l} / \mathrm{sec})$ on the syringe pump while removing fluid from the rotating tube to prevent disturbance of the cells in the tube. The maximum sample volume that could be removed from the rotating tube, without significantly affecting the steady rotation of the sample, was determined to be $200 \mu \mathrm{l}$. At the end of the ferrofluid removal process, the syringe pump and rightmost pipette were used to remove the processed sample from the conical tube and to deposit it in a CellSearch cartridge for subsequent analysis. A control computer, which is part of the AutoPrep instrument, running Linux and indicated by part G, was used to control the syringe pump and pipetting arm. Several custom protocols were written to perform priming of the fluidic system, purging of the fluidic system, removal of a defined volume from the conical tube, and transfer of a sample to the CellSearch cartridge.

\subsubsection{CELLTRACKS ANALYZER II AND CELLSPOTTER}

The CellTracks Analyzer II and CellSpotter are four color semi-automated fluorescence microscopes (Veridex LLC, Raritan, NJ, USA) [7]. The instruments are equipped with a $100 \mathrm{~W}$ mercury arc lamp as a light source and a motorized filter selector containing 4 filter cubes for the fluorescent dyes DAPI, PE, FITC and APC. A $10 \times / 0.45$ NA microscope objective in combination with a 12-bit monochrome CCD camera is used to acquire fluorescence images with a resolution of $0.645 \mu \mathrm{m} /$ pixel covering the entire surface of the sample chamber. The acquired images are analyzed for events that are positive for both Cytokeratin-PE (CK-PE) and DAPI and a gallery of all those events is shown to the operator. An expert reviewer then applies several criteria to determine whether or not an event classifies as being a CTC. These criteria require the event to have a diameter of at least 4 $\mu \mathrm{m}$, round to oval morphology, a clearly defined nucleus, positive staining for CK-PE and negative staining for CD45-APC. CD45-APC is used as a negative marker to avoid classification of leukocytes nonspecifically staining with CK-PE as CTC.

The CellTracks Analyzer II was used to process cartridges containing samples from cancer patients that were and were not processed for removal of free ferrofluid. The number of CTC in both types was determined by an expert reviewer using the criteria outlined above. The Cellspotter was used to analyze samples containing SKBR-3 cells spiked in buffer and SKBR-3 and PC3-9 cells spiked in whole blood. 
TABLE 7.1 : Overview of median diameter and standard deviation of cell populations used in the simulation.

\begin{tabular}{llll}
\cline { 2 - 4 } & Leukocyte & $7.8 \mu \mathrm{m}$ & $1.8 \mu \mathrm{m}$ \\
0 & SKBR-3 & $16.9 \mu \mathrm{m}$ & $2.7 \mu \mathrm{m}$ \\
& PC3-9 & $19.5 \mu \mathrm{m}$ & $3.6 \mu \mathrm{m}$ \\
& CTC-B & $13.3 \mu \mathrm{m}$ & $3.5 \mu \mathrm{m}$ \\
\hline
\end{tabular}

\subsection{REsults}

\subsubsection{SIMULATION OF PARTICLE MOVEMENT IN CONICAL TUBE}

A simulation of the movement of free EpCAM-FF in solution, leukocytes, SKBR-3 cells, PC3-9 cells, and CTC from patients with breast cancer (CTC$\mathrm{B})$ and prostate cancer (CTC-P) was written and conducted in Matlab (Mathworks, Natick, MA, USA). All cell types, except leukocytes, were simulated both with and without EpCAM-Ferrofluid attached to the cell. This simulation was developed to determine the influence of cell size and whether or not a cell is labeled with EpCAM-FF on its location over time in the rotating conical tube. Finally, the results from the simulation were compared to experiments to validate the results. The main parameters that were used in the simulation are cell diameter and cell density. Cell diameters for leukocytes, SKBR-3 and PC3-9 cells were determined by coulter pipette and cell diameters for CTC-B and CTC-P were determined from image analysis of CTC detected by the CellSearch system [9]. The results are shown in Table 7.1.

A diameter of $170 \mathrm{~nm}$ was used for ferrofluids. Cell density was 1080 $\mathrm{kg} / \mathrm{m}^{3}$ for all cell types that were simulated without ferrofluids attached to the cell. The average number of EpCAM binding sites on the cell surface is approximately $5 \times 10^{5}$ for SKBR-3 cells and $5 \times 10^{4}$ for PC3-9 cells, CTC-B and CTC-P [5]. To determine the number of ferrofluids to simulate, the maximum number of ferrofluids was calculated that can fit around a cell of a certain diameter assuming a maximum hexagonal packing coefficient for spheres on an approximately flat surface of 0.907 . The maximum number of ferrofluids were: SKBR-3 cells: $3.66 \times 10^{4}$, PC3-9 cells: $4.86 \times 10^{4}$, CTC-B: $2.28 \times 10^{4}$ and CTC-P: $1.57 \times 10^{4}$. Therefore, the number of EpCAM binding sites is significantly larger than the number of ferrofluids that can fit around a CTC-B, CTC-P or SKBR-3 cell. For PC3-9 cells, the number of EpCAM binding sites is approximately equal to the maximum number of ferrofluids that fit around the cell. For the simulation we assumed that all cells are labeled with the maximum number of ferrofluids that fit around it.

The binding of EpCAM-FF increases the effective density of the simu- 
lated cell types with respect to the density of $1080 \mathrm{~kg} / \mathrm{m}^{3}$ for cells without ferrofluid labeling. Effective cell density after ferrofluid labeling was calculated as shown in Equation (7.1).

$$
\rho_{\text {cell }+F F}=\frac{\left(N_{F F} \cdot m_{F F}\right)+\left(\rho_{\text {cell }} \cdot V_{\text {cell }}\right)}{\left(N_{F F} \cdot V_{F F}\right)+V_{\text {cell }}}
$$

In the equation above, $\mathrm{N}_{\mathrm{FF}}$ is the number of ferrofluids on a cell, with $m_{F F}$ and $V_{F F}$ being the mass and volume of a single ferrofluid particle. $\mathrm{V}_{\text {cell }}$ and $\rho_{\text {cell }}$ are the volume and density of the unlabeled cell. For SKBR-3 cells the calculated density is $1232 \mathrm{~kg} / \mathrm{m}^{3}$, for PC3-9 cells $1279 \mathrm{~kg} / \mathrm{m}^{3}$, for CTC-B $1176 \mathrm{~kg} / \mathrm{m}^{3}$ and for CTC-P $1146 \mathrm{~kg} / \mathrm{m}^{3}$. These values were used to simulate SKBR-3 cells, PC3-9 cells, CTC-B and CTC-P that are fully labeled with EpCAM-FF.

For each cell type, 1000 cells were simulated for a period of 1200 seconds. To simplify the simulation, we assumed that all cell types and ferrofluids are perfect spheres. To avoid the combined use of the terms cell, leukocyte, CTC-B or CTC-P, SKBR-3 or PC3-9 cell, we describe each as a "particle".

Three main forces are present for a particle in a liquid: the gravitational force, buoyancy force and the drag force induced by the vertical movement of the particle through the fluidic medium which is said to have a density of $1000 \mathrm{~kg} / \mathrm{m}^{3}$. Upon centrifugation of the conical tube, a fourth force becomes active: a centrifugal force that moves the particles to the outside of the rotating tube. The drag force limits the maximum sinking- and sideward speed of the particle in the fluidic medium. We used the Stokes law to determine the drag on cells and ferrofluids, which is valid for Reynolds numbers less than 1. Calculations show that the Reynolds number for the largest SKBR-3 cell that is simulated is 0.02 , satisfying the above criterion. The simulation starts by randomly distributing particles, either cells or ferrofluids, throughout the volume that is occupied by a sample of $360 \mu \mathrm{l}$. The first part of the simulation uses the cylindrical coordinate system. All forces, except the drag force, are only active in either the vertical or radial direction. Therefore, the drag force was split into its vertical and radial components. The resulting five forces and associated dimensions are given in Equation (7.2) - (7.6) and shown in Figure 7.4.

$$
\begin{gathered}
F_{\text {gravity }}=m \cdot g \\
F_{\text {bouyancy }}=\rho_{\text {buffer }} \cdot V \cdot g \\
F_{\text {centrifugal }}=m \cdot \omega^{2} \cdot R \\
F_{\text {drag }, Z}=6 \cdot \pi \cdot \eta \cdot r \cdot v_{Z}
\end{gathered}
$$


A

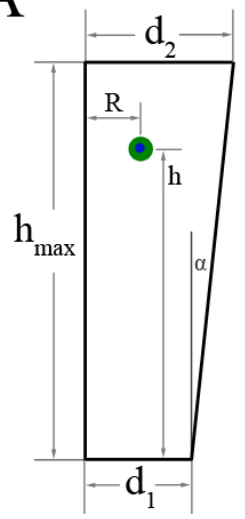

$\mathrm{B}$

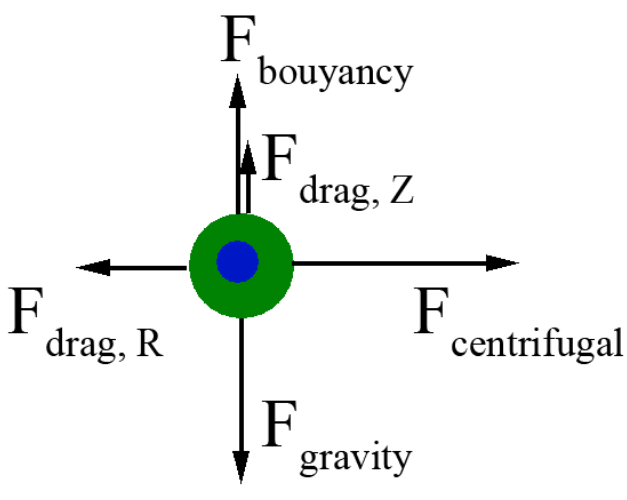

Figure 7.4 : (A) Cross-sectional view of the part of the tube that is occupied by a $360 \mu \mathrm{l}$ sample. The center of the conical tube is coincident with the left vertical line and the sloped outer wall is at the right. Parameters represent: $\mathrm{h}_{\max }=$ height of $360 \mu \mathrm{l}$ sample $(10.44 \mathrm{~mm}), \mathrm{d}_{1}=$ radius of tube at bottom $(2.75 \mathrm{~mm}), \mathrm{d}_{2}=$ radius of tube at upper surface of a $360 \mu \mathrm{l}$ sample $(3.85 \mathrm{~mm}), \alpha=$ angle of tube wall to vertical $\left(6^{\circ}\right), \mathrm{R}=$ distance of particle from center of tube and $h=$ height of particle above bottom of tube. (B) Overview showing the forces that act on a particle while the tube it is in is being centrifuged. The particle is assumed to be displaced from the exact center of the tube and the center of the tube is located to the left of the particle.

TABLE 7.2 : Overview and description of symbols used in equations with their corresponding units of measurement.

\begin{tabular}{lll}
\hline Symbol & Description & Unit \\
\hline $\mathrm{m}$ & Mass of particle & $\mathrm{kg}$ \\
$\mathrm{g}$ & Acceleration of gravity & $\mathrm{m} / \mathrm{s}^{2}$ \\
$\rho$ & Density of medium or particle & $\mathrm{kg} / \mathrm{m}^{3}$ \\
$\mathrm{~V}$ & Volume of particle & $\mathrm{m}^{3}$ \\
$\omega$ & Angular velocity of conical tube & $\mathrm{s}^{-1}$ \\
$\mathrm{R}$ & Distance of particle to the center of the tube & $\mathrm{m}$ \\
$\eta$ & Viscosity of medium & $\mathrm{Pa} \cdot \mathrm{s}$ \\
$\mathrm{v}_{z}$ & Velocity of particle in the vertical direction & $\mathrm{m} / \mathrm{s}$ \\
$\mathrm{V}_{\mathrm{R}}$ & Velocity of particle in the radial direction & $\mathrm{m} / \mathrm{s}$ \\
\hline
\end{tabular}

$$
F_{d r a g, R}=6 \cdot \pi \cdot \eta \cdot r \cdot v_{R}
$$

All five forces and components of forces act on both the cells and the ferrofluids in the sample while not in contact with the outer wall of the tube. The terms that are present in the equations above are shown in Table 7.2 . 
The model that is described by the five equations was iterated in Matlab using small time-steps, $\Delta \mathrm{t}$, of $5 \mu \mathrm{s}$ (for cells) and $10 \mathrm{~ns}$ (for ferrofluids). The initial radial position of the particle is used to calculate the initial centrifugal force on the object. This results in an initial acceleration using $\mathrm{F}=\mathrm{m} \cdot \mathrm{a}$. The final speed of the particle in the radial direction is calculated according to Equation (7.7).

$$
v_{R, \text { final }}=v_{R, \text { initial }}+\left(\frac{F_{\text {centrifugal }}-F_{\text {drag }, R}}{m}\right) \cdot \Delta t
$$

The same calculation is used to determine the initial acceleration due to gravity and the resulting initial speed of the particle in the vertical direction after one time step. However, this can be simplified by assuming that the particle always moves at its terminal velocity. This terminal velocity is reached when the gravitational force minus the buoyancy force equals the drag force in the vertical direction. Combining equations (7.2), (7.3) and (7.5) and simplifying results in Equation (7.8).

$$
v_{Z, \text { particle }}=\frac{2 \cdot r_{\text {particle }}^{2} \cdot g \cdot\left(\rho_{\text {particle }}-\rho_{\text {buffer }}\right)}{9 \cdot \eta}
$$

For a typical SKBR-3 cell in a medium with a density of $1 \times 10^{3} \mathrm{~kg} / \mathrm{m}^{3}$ and a viscosity of $0.001 \mathrm{~Pa} \cdot \mathrm{s}$, this results in a terminal velocity of $35 \mu \mathrm{m} / \mathrm{s}$ in the vertical direction. The time it takes the particle to accelerate to the terminal vertical velocity was determined to be less than $100 \mu \mathrm{s}$. We therefore simplified the simulation with the assumption that all cells move downward in the rotating tube at their terminal speed, while not in contact with the wall of the tube.

Equation (7.7) shows the method that is used to calculate the radial velocity of the simulated particle. The calculated final speed is used at the start of the next time-step to update the position of the particle according to Equation (7.9).

$$
R_{\text {final }}=R_{\text {initial }}+\left(v_{R, \text { initial }} \cdot \Delta t\right)
$$

Then, the forces on the simulated particle are determined using the new position and speed and this process is repeated until the particle arrives at the outer wall of the tube, or when the simulation ends due to reaching the predetermined running time of 1200 seconds. The particle is assumed to have arrived at the outer wall when the radial position reaches the defined location of the outer wall at a certain height in the tube. From this point the simulation changes as the particle is now assumed to stay at the outer wall and move along it due to the component of the centrifugal force in the direction parallel to the outer wall. An overview of the forces which are active on the particle while at the outer wall of the tube is shown in Figure 7.5 . 


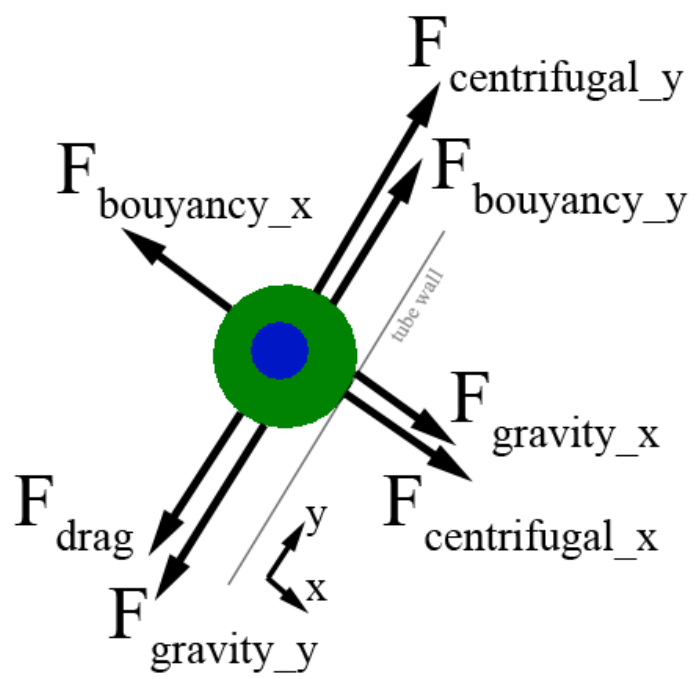

FIGURE 7.5 : Schematic drawing of components of forces active on a particle that moves along the tube wall, which is depicted by the gray line. The angle of the tube wall to the vertical $\left(6^{\circ}\right)$ is exaggerated for illustrative purposes. Forces along the x-axis are directed into or out of the tube wall. Forces along the y-axis are directed along the tube wall. Additions of either $\mathrm{x}$ or $\mathrm{y}$ indicate the axis of the force component. The coordinate axis is shown and the $y$-axis starts at the bottom of the tube and the maximum value is determined by $\mathrm{h} / \cos (\alpha)$.

The force components depicted in Figure 7.5 are calculated according to Equation (7.10) - (7.16).

$$
\begin{gathered}
F_{\text {centrifugal }, y}=\sin (\alpha) \cdot m \cdot \omega^{2} \cdot\left[d_{1}+\left(\left(\frac{y \cdot \cos (\alpha)}{h_{\max }}\right) \cdot\left(d_{2}-d_{1}\right)\right)\right] \\
F_{\text {bouyancy } y}=\cos (\alpha) \cdot \rho_{\text {buffer }} \cdot V \cdot g \\
F_{\text {centrifugal }, x}=\cos (\alpha) \cdot m \cdot \omega^{2} \cdot\left[d_{1}+\left(\left(\frac{y \cdot \cos (\alpha)}{h_{\max }}\right) \cdot\left(d_{2}-d_{1}\right)\right)\right] \\
F_{\text {gravity }, x}=\sin (\alpha) \cdot m \cdot g \\
F_{\text {gravity }, y}=\cos (\alpha) \cdot m \cdot g
\end{gathered}
$$




$$
\begin{gathered}
F_{\text {drag }}=6 \cdot \pi \cdot \eta \cdot r \cdot v_{y} \\
F_{\text {bouyancy } x}=\sin (\alpha) \cdot \rho_{\text {buffer }} \cdot V \cdot g
\end{gathered}
$$

In Equation (7.10), y denotes the distance of the particle along the tube wall measured from the bottom of the tube and in Equation (7.15), the symbol $\mathrm{v}_{\mathrm{y}}$ represents the speed of the particle along the tube wall. The particle is assumed to move along the tube wall without additional drag from the tube wall. Therefore, the drag force on the particle is only influenced by the viscosity of the medium, the radius of the particle and its speed along the tube wall. When the particle arrives at the tube wall according to the first part of the simulation, its velocity is set to 0 and the net force in the y-direction, along the tube wall, is calculated. Then, in the next time step, the velocity is calculated according to Equation (7.17) (Names of force components have been shortened for spacing.).

$$
v_{y, f i n a l}=v_{y, \text { initial }}+\left(\frac{F_{\text {centr }, y}+F_{\text {bouy }, y}-F_{\text {grav }, y}-F_{\text {drag }}}{m}\right) \cdot \Delta t
$$

The position of the cell along the tube wall is updated and then the force components are re-calculated. This process is repeated until the calculation ends when the predetermined calculation time of 1200 seconds is reached.

To be able to compare the results of the simulation to the experiments, the location of all the simulated particles is determined at the end of the simulation. Since the fluid is aspirated slowly from the bottom of the tube in the AFRS, it is assumed that the cells will slowly move down with the descending fluid-air interface. To determine what fraction of the sample is removed in a particular draw, we determined the fraction of the cells that are in the lower $200 \mu \mathrm{l}$ of the sample. The results of the simulation were compared to measurements performed with SKBR-3 cells spiked in buffer as described in the materials and methods section. Experiments were done three times for each time-point: after 5, 11.5 and 15 minutes of rotation at $900 \mathrm{rpm}$. Each sample was placed inside the AFRS and $200 \mu \mathrm{l}$ from the 360 $\mu \mathrm{l}$ sample volume was removed after the defined period of rotation. The removed sample was filled to $360 \mu \mathrm{l}$ with System Buffer and inserted into a CellSearch cartridge. Rotation of the conical tube was stopped and the remaining sample was agitated and washed with $200 \mu \mathrm{l}$ of System Buffer to collect all remaining cells. This sample was also inserted into a CellSearch cartridge. Both cartridges from each of the 9 experiments were imaged by CellSpotter and the number of SKBR-3 cells was determined in each cartridge by manual review. The percentage of cells removed was defined by the number of cells detected in the aspirated volume divided by the total number of cells detected in both the aspirated volume and the remaining sample. The results are shown in Figure 7.6. 


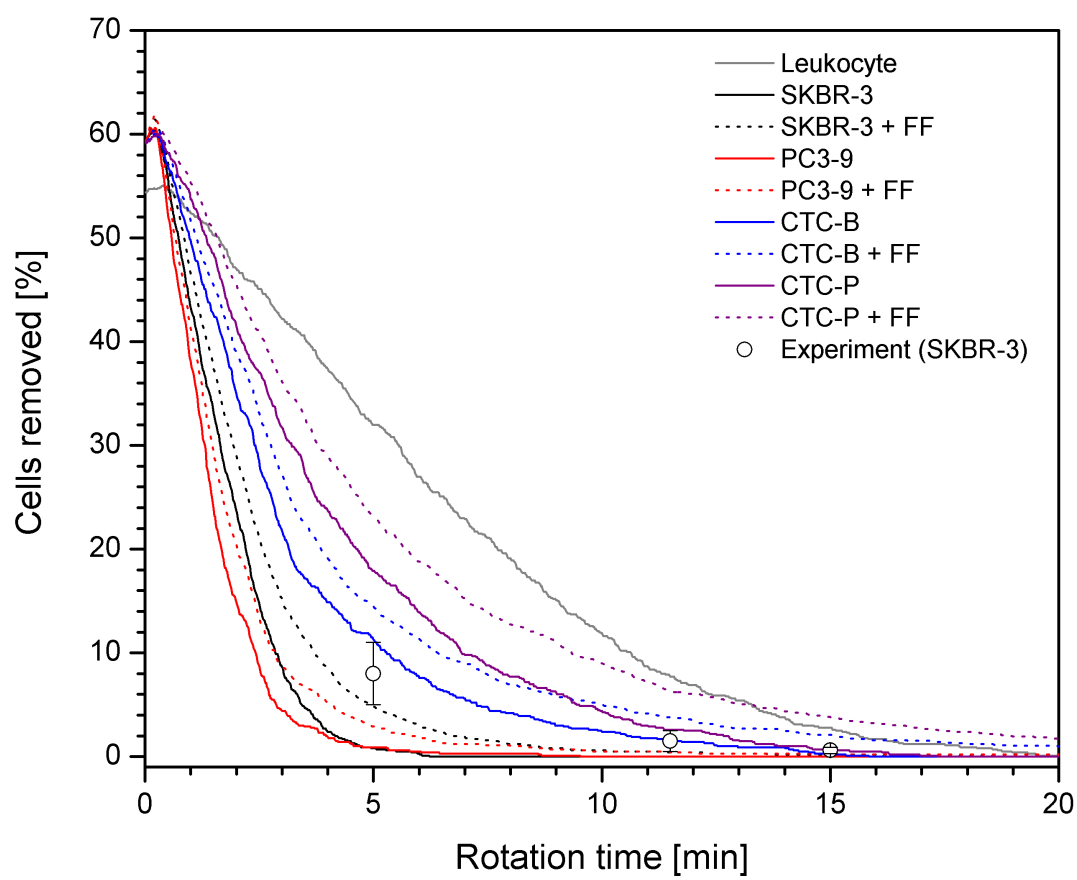

FigURE 7.6 : Simulation results of percentage of cells removed from the sample after withdrawing $200 \mu \mathrm{l}$ from the $360 \mu \mathrm{m}$ sample volume. All solid lines indicate unlabeled cells and all dotted lines indicate maximum labeling with EpCAM-FF (indicated as "+ FF"). Gray line indicates leukocytes, black lines indicate SKBR-3 cells and red lines indicate PC3-9 cells. Blue lines indicate CTC-B and purple lines indicate CTC-P. For each cell type, 1000 cells were simulated. The experiment data-points show the results of the removal of $200 \mu \mathrm{l}$ from the $360 \mu \mathrm{l}$ sample using SKBR-3 cells in buffer. For each experiment data point: $\mathrm{n}=3$ and error bars indicate 1 standard deviation.

Not shown in Figure 7.6 are the results of the simulation of free ferrofluids. This simulation was more time consuming due to the much smaller time-step used. Therefore, a limited number of 25 free ferrofluids were simulated for 1200 seconds. Review of the results indicate that the median distance traveled by a single ferrofluid particle in that time is $63 \mu \mathrm{m}$ in the vertical direction and $136 \mu \mathrm{m}$ in the radial direction with a standard deviation of $128 \mu \mathrm{m}$. The low values of these distances as compared to the dimensions of the conical tube support the assumption that free ferrofluids stay randomly distributed in the sample volume. The results shown in Figure 7.6 indicate that, due to their size, leukocytes are expected to be removed in a greater percentage as compared to the larger SKBR-3 cells, up to a rotation time of $20 \mathrm{~min}$. Also, the increase in density of SKBR-3 cells due to labeling with EpCAM-FF causes a significant increase in their 
removal from the rotating sample up to 10 minutes of rotation. For CTC-B and CTC-P, the expected loss of cells per withdrawal step decreases to $1.0 \%$ and $1.8 \%$ after 20 minutes of rotation. The expected recovery after this time is greater than $90 \%$ for both CTC-B and CTC-P, when 6 withdrawal steps are used.

\subsubsection{DETERMINATION OF FREE FERROFLUID REMOVAL EFFICIENCY}

The free ferrofluid that is present in the sample remains approximately randomly distributed as was confirmed by the simulations in the previous section. The fraction of ferrofluid that is removed is therefore expected to scale with the fraction of the sample that is removed. To validate the ferrofluid removal efficiency, we prepared 7 samples containing SKBR-3 cells in buffer and free ferrofluid at a concentration of $40 \mu \mathrm{g} / \mathrm{ml}$. For each sample, $200 \mu \mathrm{l}$ of the $360 \mu \mathrm{l}$ sample volume was withdrawn after 20 minutes of rotation. The withdrawn amount was replaced by System Buffer and this completed the first withdrawal step. This process was repeated until six withdrawal steps were processed. The $200 \mu \mathrm{l}$ aliquots that were withdrawn were analyzed on the spectrophotometer to determine their absorption. From the obtained absorption values we determined the corresponding concentration of free ferrofluid using the calibration graph. The fraction of EpCAM-FF removed in each successive withdrawal step was determined by dividing the measured concentration of EpCAM-FF by the initial concentration of $40 \mu \mathrm{g} / \mathrm{ml}$. The results are shown in Figure 7.7.

The number of withdrawal steps that is required to remove $>95 \%$ of free EpCAM-FF is 4 based on random distribution of Ferrofluid in the rotating tube at all times. However, as is apparent from Figure 7.7, the actual EpCAM-FF removal efficiency is somewhat lower, especially after the first 2 withdrawal steps. Averaging over all 42 measurements, we calculated the efficiency to be $76.6 \%$. By factoring in this efficiency, the number of withdrawal steps necessary for removal of $>95 \%$ of free EpCAM-FF was determined to be 6 . This value was used in the experiments that were performed with spiked tumor cells in buffer and whole blood of healthy donors and in blood samples from cancer patients.

\subsubsection{SKBR-3 CELLS IN BUFFER}

The cell recovery and EpCAM-Ferrofluid removal efficiency of the AFRS were determined using samples containing SKBR-3 cells in buffer. Samples were prepared as described in the materials and methods section. Samples that were not to be processed for removal of free ferrofluid were transferred to a CellSearch cartridge and placed inside a CellSearch Magnest and imaged using the CellSpotter system. Samples that were to be processed for removal of free ferrofluid were transferred to a conical tube. The tube was lightly agitated to achieve random distribution of cells and placed in 


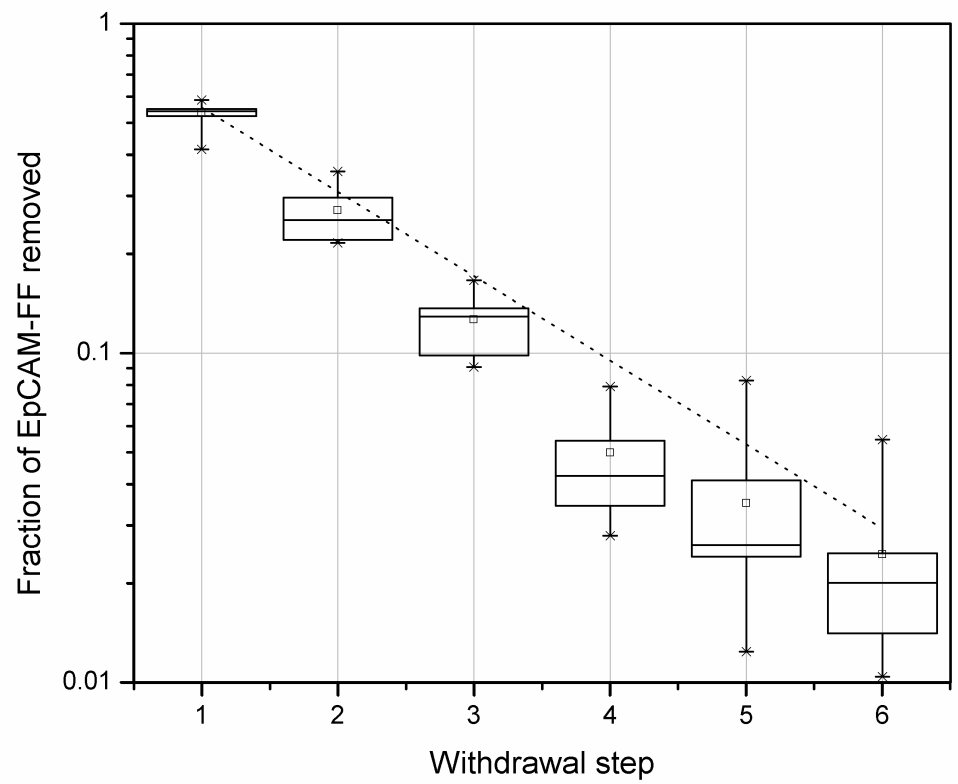

FiguRE 7.7 : Boxplots showing the fraction of EpCAM-FF removed from a sample containing SKBR-3 cells in buffer for each successive withdrawal step. Each boxplot shows the distribution of EpCAM-FF that was removed for that specific draw number. In each boxplot, the box indicates first to third quartile, solid line indicates median and small squares indicates mean, whiskers show minimum and maximum values. Dotted line indicates theoretical maximum EpCAM-FF removal for random distribution.

the already primed AFRS. The system was run using 6 withdrawal steps, with a period of rotation of 20 minutes before each withdrawal step. The final processed sample was transferred to a CellSearch cartridge and placed inside a CellSearch Magnest and imaged using the CellSpotter system. After processing of the recorded images using a custom ImageJ script, the number of cells was determined by manual review. Some clusters, of generally 2 cells, were detected and were counted as single cells. The results are shown in Figure 7.8 .

Correlation was excellent at 0.999 and the median recovery was determined to be $97.8 \%$, with a standard deviation of $3.2 \%(\mathrm{n}=8)$. Ferrofluid removal efficiency was calculated by dividing the concentration of free ferrofluid in samples processed for removal of free ferrofluid by the concentration of free ferrofluid in samples not processed for removal of free ferrofluid. The median removal efficiency was determined to be $96.1 \%$ with 


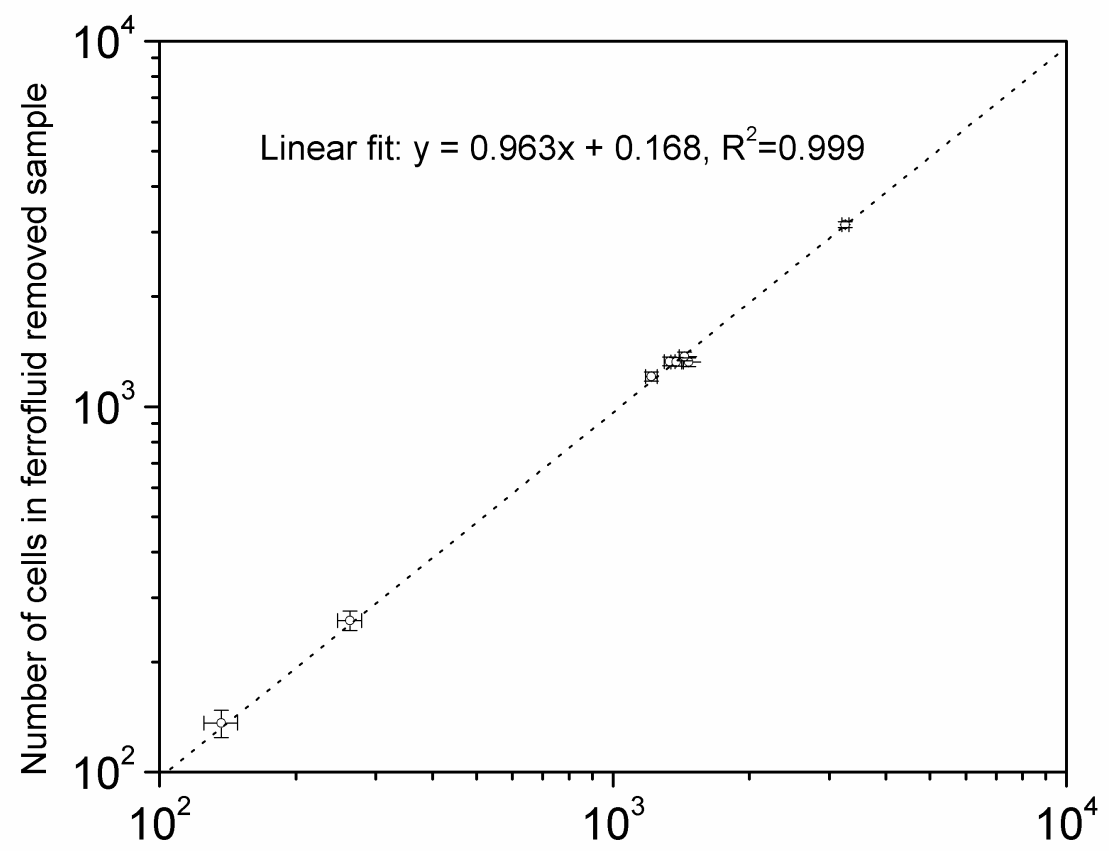

Number of cells in sample not processed for ferrofluid removal

FiguRE 7.8: Correlation of number of cells detected in samples not processed for ferrofluid removal and samples processed for ferrofluid removal. Error bars indicate square root of detected number of cells. Dotted line indicates linear fit, $\mathrm{n}=8$.

a standard deviation of $1.3 \%$.

\subsubsection{SKBR-3 AND PC3-9 CELLS SPIKED IN WHOLE BLOOD}

After the spiked whole blood samples were processed by the CellTracks AutoPrep, the conical tubes were collected and transferred to the AFRS site. This was done within 4 hours after completion of the AutoPrep protocol. AutoPrep processed samples from odd numbered tubes were used as control samples and were not processed for removal of free ferrofluid. These control samples were transferred to a CellSearch cartridge and analyzed on the CellSpotter. AutoPrep processed samples from even numbered tubes were processed for removal of free ferrofluid. The conical tubes containing these samples were agitated and shaken lightly to achieve a random distribution of cells and ferrofluids. After placement in the AFRS system, rotation of the conical tube was started and 6 ferrofluid withdrawal steps were performed after 20 minutes each. The final processed sample was transferred to a CellSearch cartridge and also analyzed using the CellSpotter system. Each 
cartridge was imaged for DAPI, Cytokeratin-PE, Her2/neu-FITC, CD45APC and bright-field. For each color, 180 images were captured and stitched together using a custom ImageJ script. Events of interest were segmented based on a fixed PE threshold of 60 and all segmented events were collected into a multipage tiff file. Quantitative parameters, for example size, total intensity and circumferences were calculated. Labview was then used to display dot-plots of selected parameters and associated images of selected cells with the her2-FITC fluorescence channel being used to discriminate between SKBR-3 and PC3-9 cells. Both single SKBR-3 and PC3-9 cells and clusters of SKBR-3 and PC3-9 cells were counted by an experienced operator. Clusters, usually consisting of 2 cells, were counted as single cells and added to the total number of detected single cells. After review of the first 12 duplicate samples, only SKBR-3 cells and no PC3-9 cells were detected. Therefore, we assumed that these cells were not present in the samples after processing by the AutoPrep instrument. The results of these samples are presented separately in Figure 7.9. The correlation is 0.857 and median recovery of SKBR-3 cells spiked in whole blood was $84.3 \%$ with a standard deviation of $22.2 \%(\mathrm{n}=12)$. Median ferrofluid removal efficiency was determined to be $95.2 \%$, with a standard deviation of $1.9 \%$.

Further processed samples from the AutoPrep that were received after the initial samples, which only contained SKBR-3 cells, did contain both cell types as expected. The number of detected SKBR-3 and PC3-9 cells in samples that were and were not processed for removal of free ferrofluid are shown in Figure 7.10. The linear fits in Figure 7.10 indicate that the correlation for SKBR-3 and PC3-9 cells in these samples was significantly lower than those observed in Figures 7.8 and 7.9. The median recovery of SKBR-3 cells $(\mathrm{n}=7)$ in samples that also contained PC3-9 cells was $71.5 \%$, with a standard deviation of $48.4 \%$, and is significantly lower than the median recovery of SKBR-3 cells in samples that only contained these types of cells. Also, the median recovery of PC3-9 cells $(n=7)$ was determined to be $68.2 \%$, with a standard deviation of $8.8 \%$, and is similar to the recovery of SKBR-3 cells. Median ferrofluid removal efficiency of samples containing both SKBR-3 and PC3-9 cells was 94.7\%, with a standard deviation of $1.7 \%$. Median ferrofluid removal efficiency of all spiked whole blood samples was $94.7 \%$, with a standard deviation of $1.8 \%$. Recovery values given above are only for the process of removal of free ferrofluid. Additional loss of initial spiked cell numbers $(\sim 15 \%)$ and increased variance already occurred due to processing of the spiked whole blood samples in the AutoPrep.

\subsubsection{SAMPLES FROM CARCINOMA PATIENTS}

Duplicate samples from 11 prostate cancer patients were processed by the AutoPrep. One half of the duplicate samples was not processed for ferrofluid removal and theses samples were transferred to CellSearch cartridges and analyzed on CellTracks Analyzer II. The other half of the duplicate samples 


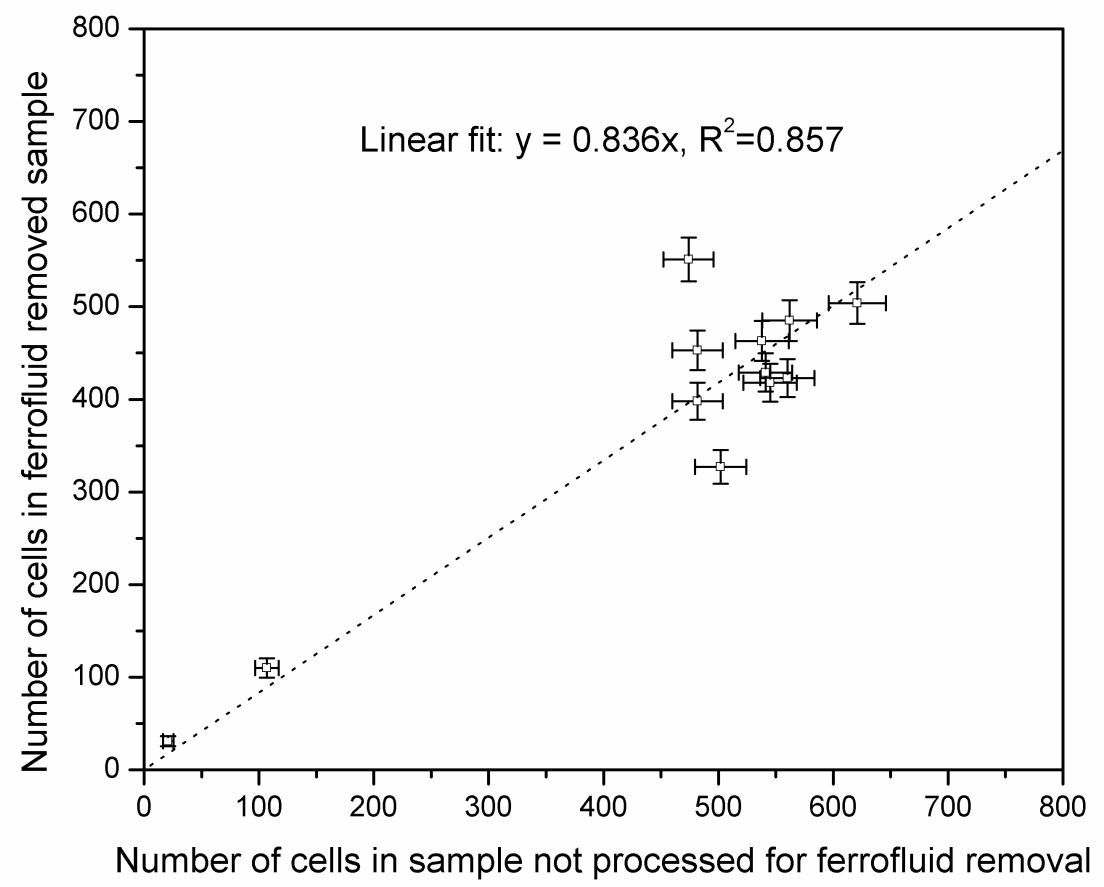

FIGURE 7.9 : Correlation of number of SKBR-3 cells detected in samples that were not processed for removal of free ferrofluid against number of SKBR-3 cells detected in samples processed by the AFRS to remove free ferrofluid. The samples that were used for this figure contained only spiked SKBR-3 cells in whole blood. Error bars indicate square root of detected number of cells, $n=12$. Dotted line indicates linear fit through zero.

was processed for removal of free ferrofluid the same way as the whole blood samples spiked with SKBR-3 and PC3-9 cells. Ferrofluid removed samples were also transferred to CellSearch cartridges and analyzed on the CellTracks Analyzer II. Number of CTC was determined by an expert reviewer based on the default CellSearch CTC criteria. The results of the detected number of CTC are shown in Figure 7.11.

The correlation of detected numbers of cells was 0.978 with a median recovery of CTC of $55.8 \%$, with a standard deviation of $33.3 \%$, which is significantly lower than the recovery of both SKBR-3 cells in buffer and PC3-9 cells in whole blood. Median ferrofluid removal efficiency was $92.4 \%$, with a standard deviation of $2.2 \%$. Recovery values given above are only for the process of removal of free ferrofluid. Additional loss of CTC ( 15\%) and an increase in the variance of number of CTC in tubes from the same patient occurred due to processing of these samples in the AutoPrep. 


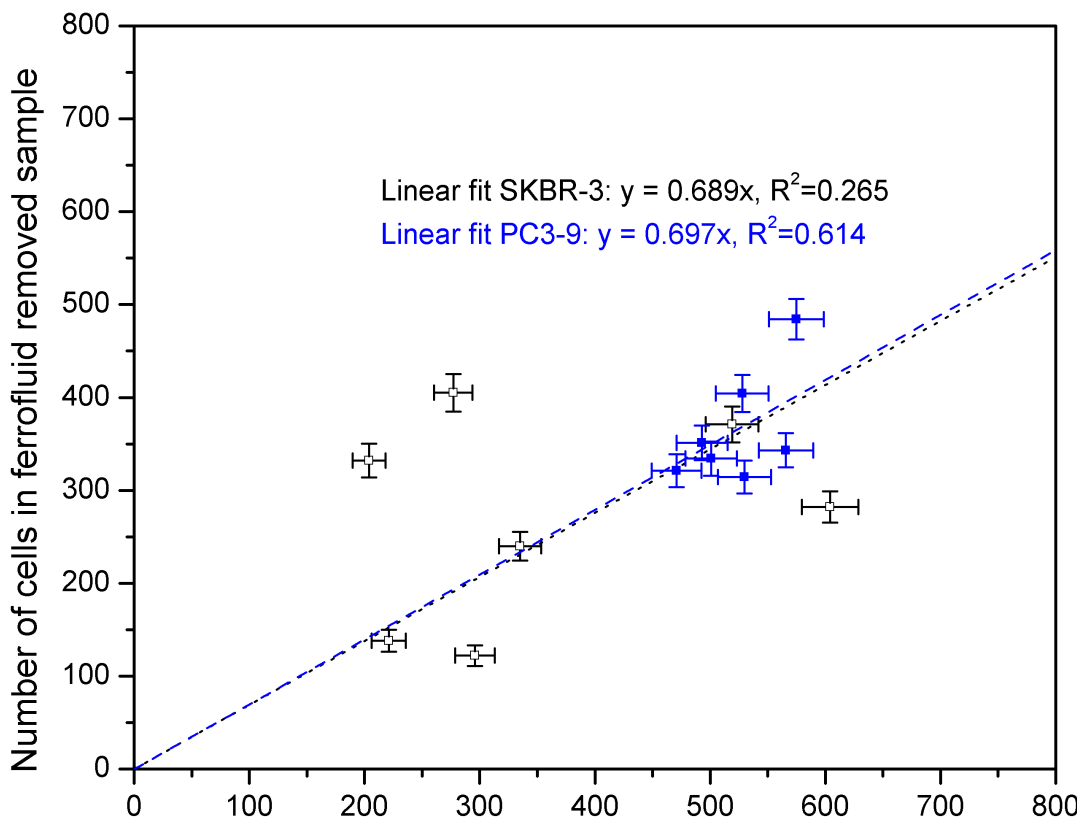

Number of cells in sample not processed for ferrofluid removal

FIgURE 7.10 : Correlation of SKBR-3 cells (black symbols) and PC3-9 cells (blue symbols) detected in samples that were and were not processed for removal of free ferrofluid. The samples that were used contained both spiked SKBR-3 and PC3-9 cells. Error bars indicate square root of detected number of cells, $\mathrm{n}=14$. Dotted black line indicates linear fit through zero for SKBR-3 cells. Dashed blue line indicates linear fit through zero for PC3-9 cells.

\subsection{Discussion}

Removal of free ferrofluid from samples enriched for CTC from $7.5 \mathrm{ml}$ of blood with EpCAM labeled ferrofluids on a CellTracks AutoPrep increases the fluorescence yield and lowers the $\mathrm{CV}$ of fluorescence detection. Additionally, it allows for the use of bright-field images to accurately determine cell outlines during image analysis, thereby increasing the specificity and detection limit of treatment targets on CTC. To remove free ferrofluid from a sample, we developed a method that uses centrifugation and careful aspiration of part of the sample, with the possibility of integration in the CellTracks AutoPrep. The automated ferrofluid removal system (AFRS) presented here was designed to remove $>95 \%$ of free ferrofluid, while keeping CTC loss to below 10\%. A simulation in Matlab was developed to determine the minimum rotation time required to achieve the above criteria. The number of withdrawal steps necessary to remove $>95 \%$ of free ferrofluid 


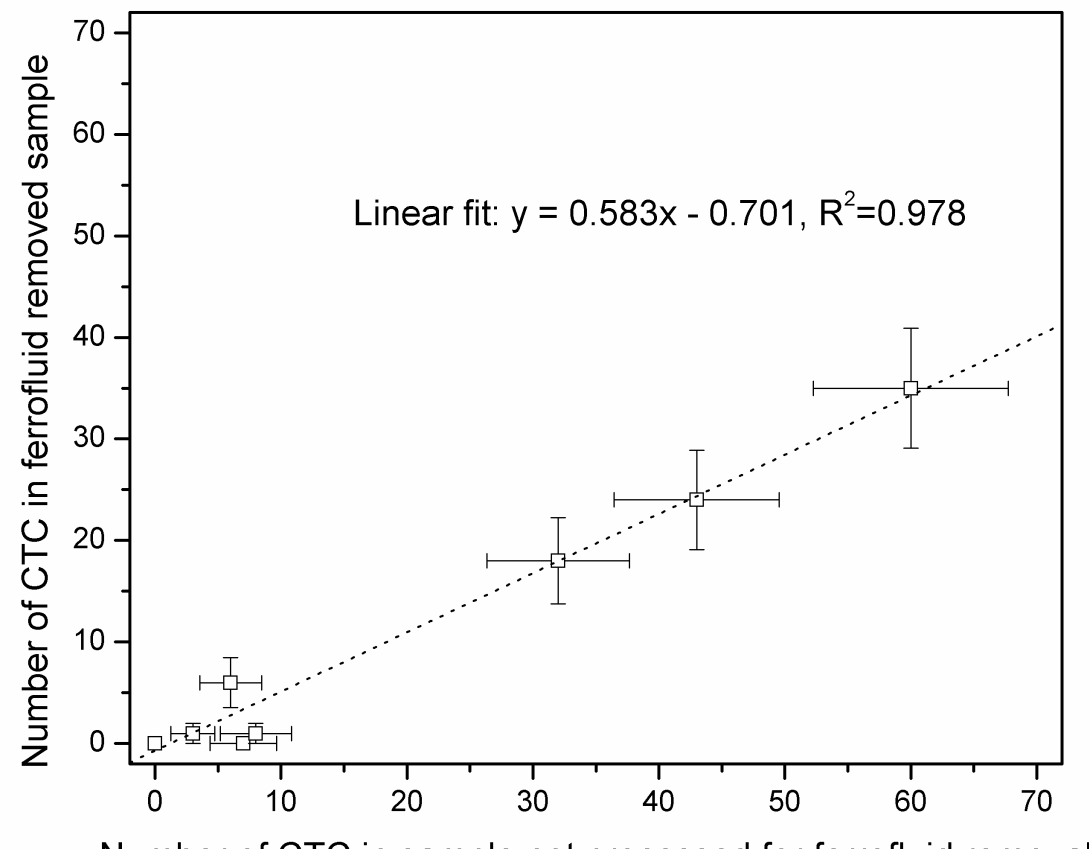

Number of CTC in sample not processed for ferrofluid removal

FIGURE 7.11 : Correlation of number of CTC detected in samples that were and were not processed for ferrofluid removal. Error bars indicate square root of detected number of cells, $\mathrm{n}=11$. Dotted black line indicates linear fit.

was determined from the concentration of free ferrofluid in successive withdrawals as measured by a spectrophotometer. Results from the simulation were compared to results of recovery of SKBR-3 cells spiked in buffer that were processed by the AFRS. Finally, samples from patients with colorectal cancer were used to test the AFRS. Results from the AFRS simulations in Matlab indicate that a larger percentage of SKBR-3 cells is removed in reality than is expected based on the results of the simulation. This discrepancy is most pronounced after a period of rotation of 5 minutes. In the experiments with SKBR-3 cells spiked in buffer, $8 \%$ of the cells were removed while a removal of $2.7 \%$ was expected based on the results of the simulation. This discrepancy might be due to a difference in the diameter of the SKBR-3 cells that was used in the simulation versus the effective diameter of SKBR-3 cells in the rotating tube. The effective diameter of a cell in the rotating tube might be lower due to hydrodynamic forces, resulting in higher cell loss. Also, while sliding along the outer wall, cells might experience some friction, were zero friction was assumed, which would reduce their velocity along the outer wall and would also result in higher 
cell loss.

In determining the efficiency of the AFRS we used SKBR-3 cells to mimic CTC that are found in breast cancer patients (CTC-B). The simulated recovery of SKBR-3 cells was higher than the simulated recovery of CTC-B, which is mainly due to the smaller size of the CTC-B as compared to the SKBR-3 cells (median diameter $13.3 \mu \mathrm{m}$ against $16.9 \mu \mathrm{m}$ ). Also, the density of EpCAM antigens on SKBR-3 cells is approximately 10-fold higher than the density of EpCAM antigens found on CTC-B. Although it is unknown to what extent the EpCAM binding sites on SKBR-3 and CTC-B are bound to ferrofluids, it is likely that more ferrofluids are bound to a SKBR-3 cell as compared to a CTC-B, resulting in a higher effective density of the SKBR-3 cell. This higher density of SKBR-3 cells results in a lower recovery, somewhat negating the difference in recovery that is caused by the difference in size between SKBR-3 cells and CTC-B. The accuracy of using spiked cells in buffer or whole blood to mimic CTC found in carcinoma patients may be improved with the use of cell lines with more similar diameters. For example, the SW-480 and COLO-320 cell lines have size distributions, which are similar to CTC found in colorectal and prostate cancer patients [9].

The design specifications for the AFRS consisted of a recovery of CTC greater than $90 \%$ and a removal efficiency of free ferrofluid greater than $95 \%$. Results of buffer samples spiked with SKBR-3 cells exceeded these specifications at a median cell recovery of $97.8 \%$ and a median free ferrofluid removal efficiency of $96.1 \%$. Removal efficiency of free ferrofluid in whole blood spiked with SKBR-3 and PC3-9 cells is close to the design specification at $94.7 \%$. However, the recovery of cells was well below $90 \%$ for these samples at a median recovery of $76.6 \%$. This is lower than the recovery that is observed in samples containing SKBR-3 cells in buffer. After processing of the spiked whole blood samples in the AutoPrep, lymphocytes and granulocytes are present in the final sample, next to the spiked cells. The presence of these cells, which were not simulated, may have disturbed the movement of the spiked cells, lowering their average velocity and thereby increasing the loss of both the spiked SKBR-3 and PC3-9 cells. Also, the correlation of the linear fit, shown in Figure 7.10, was significantly lower for SKBR-3 cells than for PC3-9 cells. This can be explained by the observation of a degraded SKBR-3 cell structure in which a majority of the cells had an apoptotic phenotype and a larger than normal percentage of cells was clustered. These degraded cells were observed in 3 of the latter samples, which were used in Figure 7.10 and the observed degraded cell structure is most likely due to the quality of the SKBR-3 cells that was used to spike the whole blood samples. The large variation of the detected number of cells in these samples and therefore the low correlation of the linear fit is due to uncertainty in manual classification of these degraded SKBR-3 cells by the expert reviewer.

The median number of cells that was detected in samples that were not processed for removal of free ferrofluid was 520, which is significantly lower 
than the expected number of $\sim 1000$ SKBR-3 cells in each sample. With an assay recovery of $85 \%$ [4], some cell loss is expected during processing in the AutoPrep. However the number of detected cells is lower than what was anticipated. This is most likely due to inaccuracies in the determination of the cell concentration in the SKBR-3 stock solution. Also, 2 pairs of samples contained significantly fewer cells than the median number of detected cells. This is most likely due to a pipetting error during spiking of the SKBR-3 cells in whole blood.

Results of ferrofluid removal experiments carried out with samples from prostate cancer patients show a removal efficiency of free ferrofluid of $94.2 \%$, which is close to the specification of $>95 \%$. The achieved reduction of free ferrofluid in these samples resulted in an improved fluorescence yield and also resulted in an image of the CTC in which more detail in the fluorescence channel was discernible. Also, the bright-field image of a cell could now be used to accurately determine the cell outline, which is useful for the analysis of e.g. her2 fluorescence on CTC from breast cancer patients, which is known to be highly heterogeneous. However, the recovery of CTC in these patient samples was well below the specification at $55.8 \%$. This may be attributed to a difference in real CTC density as compared to the values used in the simulation, which were used to optimize the AFRS settings. A higher CTC density will result in increased removal. Also, the number of samples used in these experiments was unfortunately limited. In addition to the loss of CTC that occurred during removal of free ferrofluid, $\sim 15 \%$ of CTC is lost due to processing of the samples in the CellTracks AutoPrep. In conclusion, the performed experiments indicate that the AFRS is effective in removing enough free ferrofluid to optimize imaging of CTC but processed samples show an unacceptable overall loss of CTC.

Performance of the AFRS can be improved by using optimized settings such as a longer rotation period to further lower the percentage of cells that is removed in a withdrawal step. The AFRS can also be improved by e.g. customizing the conical tube to optimize cell movement or the addition of a filter to the aspiration probe, preventing aspiration of CTC. Also, the actual density of CTC and the actual number of ferrofluids bound to a cell will need to be established to increase the accuracy of the simulation and to determine whether further improvement is feasible.

An improved method can also be developed which reduces the loss of CTC and ideally can be combined with the AutoPrep instrument. A possibility might be the use of a filter to separate CTC from ferrofluids based on their size [9]. This method will most likely remove all free ferrofluid due to the ability to repeatedly wash the filter. However, CTC may be lost due to their small size or the inability to wash them from the filter if such is necessary for further analysis or processing of the detected CTC. Another possibility might be a method which combines the use of centrifugal force and magnetic force to separate cells from ferrofluids. Instead of aspirating a fraction of the sample, as is done in the AFRS, a magnetic rod may be 
introduced instead which induces an additional force on both cells and ferrofluids. If the centrifugal force is greater than the induced magnetic force, the cells will stay at the outer wall of the rotating tube. Ferrofluids, on which the centrifugal force is approximately a factor $2 \times 10^{5}$ smaller, will then move toward the magnetic rod and be removed from the sample. An advantage of such a method is that a single withdrawal step will most likely be enough to remove more than $95 \%$ of free ferrofluid and thereby enable the optimized imaging of CTC.

\subsection{References}

[1] M. Cristofanilli, T. Budd, M. Ellis, A. Stopeck, J. Matera, M. Miller, J. Reuben, G. Doyle, W. Allard, L. Terstappen, and D. Hayes, "Circulating tumor cells, disease progression, and survival in metastatic breast cancer," $N$ Engl J Med, vol. 351(8), pp. 781-791, 2004.

[2] S. Cohen, C. Punt, N. Iannotti, B. Saidman, K. Sabbath, N. Gabrail, J. Picus, M. Morse, E. Mitchell, C. Desch, M. Miller, G. Doyle, H. Tissing, L. Terstappen, and N. Meropol, "The relationship of circulating tumor cells to tumor response, progression-free survival, and overall survival in patients with metastatic colorectal cancer," Journal of Clinical Oncology, vol. 26(19), pp. 3213-3221, 2008.

[3] J. De Bono, H. Scher, R. Montgomery, C. Parker, M. Miller, H. Tissing, G. Doyle, L. Terstappen, K. Pienta, and D. Raghavan, "Circulating tumor cells predict survival benefit from treatment in metastatic castration resistant prostate cancer," Clin Can Res, vol. 14(19), pp. 6302-6309, 2008.

[4] W. Allard, J. Matera, M. Miller, M. Repollet, M. Connelly, C. Rao, A. Tibbe, J. Uhr, and L. Terstappen, "Tumor cells circulate in the peripheral blood of all major carcinomas but not in healthy subjects or patients with nonmalignant diseases," Clinical Cancer Research, vol. 10(20), pp. 6897-6904, 2004.

[5] C. Rao, D. Chianese, G. Doyle, M. Miller, T. Russell, R. Sanders, and L. Terstappen, "Expression of epithelial cell adhesion molecule in carcinoma cells present in blood and primary and metastatic tumors," Int J Oncology, vol. 27(1), pp. 49-57, 2005.

[6] G. Attard, A. Reid, T. Yap, F. Raynaud, M. Dowsett, S. Settatree, M. Barrett, C. Parker, V. Martins, E. Folkerd, J. Clark, C. Cooper, S. Kaye, D. Dearnaley, G. Lee, and J. De Bono, "Phase I clinical trial of a selective inhibitor of cyp17, abiraterone acetate, confirms that castration-resistant prostate cancer commonly remains hormone driven," Journal of Clinical Oncology, vol. 26(28), no. 28, pp. 4563-4571, 2008.

[7] F. Coumans and L. Terstappen, Detection and characterization of Circulating Tumor Cells by the Cell Search approach, ch. -, pp. -. Humana Press, USA, 2012.

[8] M. Kagan, D. Howard, T. Bendele, J. Mayes, J. Silvia, M. Repollet, J. Doyle, J. Allard, N. Tu, T. Bui, T. Russell, C. Rao, M. Hermann, H. Rutner, and L. Terstappen, "A sample preparation and analysis system for identification of circulating tumor cells," J Clinical Ligand Assay, vol. 25(1), pp. 104-110, 2002.

[9] F. Coumans, G. van Dalum, M. Beck, and L. Terstappen, "Filter requirements for circulating tumor cell enrichment and detection," Submitted for publication, -. 


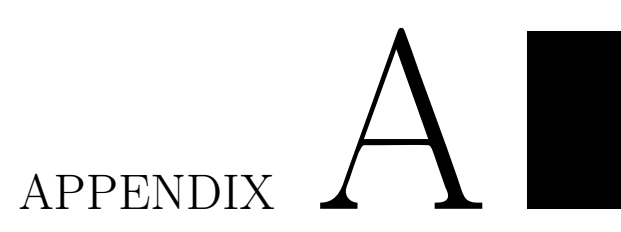

\section{OVERVIEW OF CLEAN-ROOM PROCESSING STEPS}

This appendix gives an overview of the processing steps that are involved in creating the V-Groove (Table A.1), Chimney V-Groove (Table A.2) and U-Groove (Table A.3) microstructures. The processing steps are shown schematically and are described in more detail in the following tables. 
TABLE A.1 : Flat-top V-Groove microstructures

\begin{tabular}{|c|c|c|c|}
\hline Step & Visualization & Side & Description \\
\hline 1 & & Front & $\begin{array}{l}\text { Silicon } \operatorname{DSP}^{a} \text { wafer, diameter }=100 \\
\mathrm{~mm},\left\langle 100>^{b}, 1-10 \mathrm{ohm} \cdot \mathrm{cm}, \mathrm{d}^{c}=520\right. \\
\mu \mathrm{m}\end{array}$ \\
\hline 2 & & Front & Wet Oxide layer, $500 \pm 50 \mathrm{~nm}$ \\
\hline 3 & & Front & $\begin{array}{l}\mathrm{BHF}^{d} \text { etch oxide layer with V-Groove } \\
\text { etch mask }\end{array}$ \\
\hline 4 & & Front & $\begin{array}{l}\mathrm{KOH}^{e} \text { etching of } \mathrm{V} \text {-Grooves @ } 1 \\
\mu \mathrm{m} / \mathrm{min}\end{array}$ \\
\hline 5 & & Front & Remove remaining $\mathrm{SiO}_{2}$ with $\mathrm{BHF}$ etch \\
\hline 6 & & Back & $\begin{array}{l}\text { Anodic bonding of borosilicate glass } \\
\text { wafer to backside }\end{array}$ \\
\hline
\end{tabular}

$\bar{a}$ Double side polished ${ }^{b}$ Crystallographic orientation $\quad{ }^{c}$ Thickness of wafer ${ }^{d}$ Buffered hydrofluoric acid ${ }^{e}$ Potassium hydroxide 
TABle A.2 : Chimney V-Groove microstructures

\begin{tabular}{|c|c|c|c|}
\hline Step & Visualization & Side & Description \\
\hline 1 & $\sum$ & Front & $\begin{array}{l}\text { Silicon } \mathrm{SSP}^{a} \text { wafer, diameter }=100 \mathrm{~mm}, \\
<100>^{b}, 1-10 \mathrm{ohm} \cdot \mathrm{cm}, \mathrm{d}^{c}=520 \mu \mathrm{m}\end{array}$ \\
\hline 2 & $\sum \sqrt{1} \sqrt{2}$ & Front & $\begin{array}{l}\text { DRIE }^{d} \text { etch with DRIE slots etch mask, } \\
\text { depth }=20 \mu \mathrm{m}, \text { width }=2-3 \mu \mathrm{m}\end{array}$ \\
\hline 3 & चே & Front & $\begin{array}{l}\text { Low pressure } \mathrm{CVD}^{e} \text { of } \mathrm{Si}_{3} \mathrm{~N}_{4} \text {, thickness } \\
=1.65 \mu \mathrm{m}\end{array}$ \\
\hline 4 & $\sum \sqrt{1}$ & Front & $\begin{array}{l}\mathrm{RIE}^{f} \text { etch of } \mathrm{Si}_{3} \mathrm{~N}_{4} \text { film until clearance } \\
\text { of silicon }\end{array}$ \\
\hline
\end{tabular}

$5 \sum$ Front DRIE etch of silicon, depth $=15 \mu \mathrm{m}$

$6 \sum$ Front $\mathrm{KOH}$ anisotropic V-Groove etch

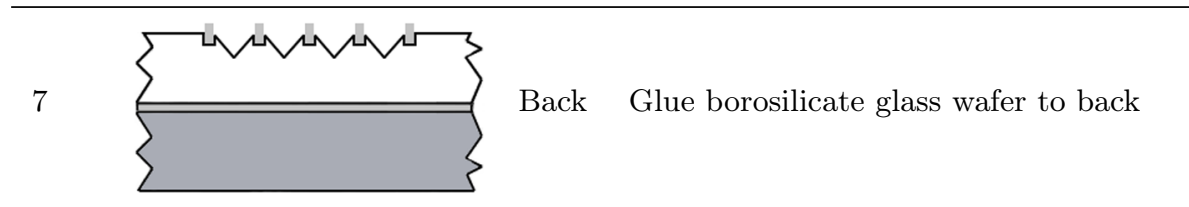

$\bar{a}$ Single side polished ${ }^{b}$ Crystallographic orientation ${ }^{c}$ Thickness of wafer ${ }^{d}$ Deep reactive ion etching ${ }^{e}$ Chemical vapor deposition ${ }^{f}$ Reactive ion etching 
TABle A.3 : U-Groove microstructures

\begin{tabular}{|c|c|c|}
\hline Step & Visualization & Side \\
\hline
\end{tabular}

158

$1 \sum\left\{\begin{array}{l}\text { Front } \begin{array}{l}\text { Silicon } \mathrm{DSP}^{a} \text { wafer, diameter }=100 \\ \mathrm{~mm},<100>^{b}, 1-10 \mathrm{ohm} \cdot \mathrm{cm}, \mathrm{d}^{c}=520 \\ \mu \mathrm{m}\end{array}\end{array}\right.$

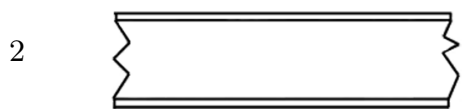

Front Wet oxidation, $1500 \mathrm{~nm} \mathrm{SiO}_{2}$, mask for $\mathrm{DRIE}^{d} / \mathrm{KOH}^{e}$ etching

3
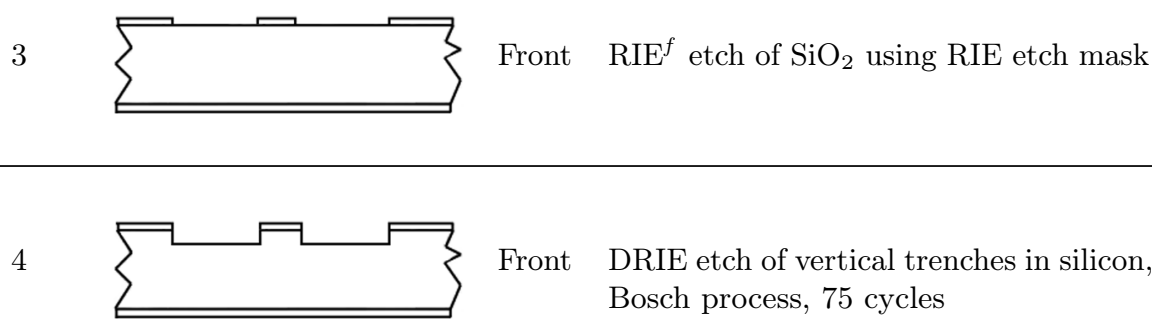

Front DRIE etch of vertical trenches in silicon, Bosch process, 75 cycles

5

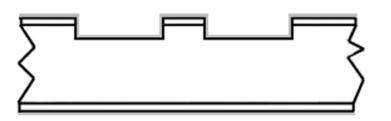

Front Thermal $\mathrm{SiO}_{2}, 1200 \mathrm{~nm}$

6

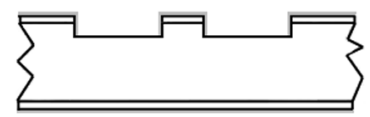

Front RIE etch of $\mathrm{SiO}_{2}$ on bottom of trenches

7

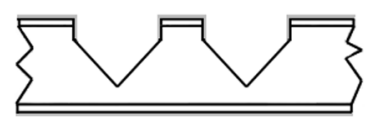

Front

$\mathrm{KOH}$ etching of $\mathrm{V}$-Groove using $25 \%$ $\mathrm{KOH}$ at $75^{\circ} \mathrm{C}$ for $70 \mathrm{~min}$
8

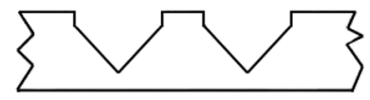

Front Strip $\mathrm{SiO}_{2}$ and apply Teflon coating (perfluoroalkylsilane deposition)

9

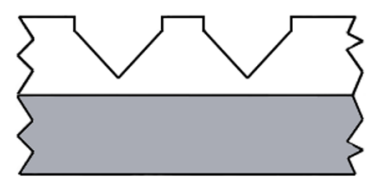

Back Glue borosilicate glass wafer to back

$\bar{a}$ Double side polished ${ }^{b}$ Crystallographic orientation ${ }^{c}$ Thickness of wafer ${ }^{d}$ Deep reactive ion etching $e$ Potassium hydroxide ${ }^{f}$ Reactive ion etching 


\section{SUMMARY}

\section{Conclusions}

The enumeration and characterization of circulating tumor cells (CTC), to improve treatment of cancer patients, is an emerging tool for the disease management of patients with metastatic carcinomas. The detection of CTC is however hampered by the very low frequency of these cells in the blood of cancer patients. The CellSearch system is the first clinically validated system for CTC detection and this thesis describes the results of research that was carried out to further improve this system.

Chapter 1 gives an introduction to cancer in general and CTC in particular. CTC are correlated to progression free- and overall survival in several types of metastatic cancers, and can be used to stratify patients in low- or high risk groups. CTC also outperform radiological imaging methods, which are still widely used in the clinic today, in terms of e.g. correlation with overall survival. Several new CTC detection technologies are described and an introduction to the CellSearch system is given, which is currently the only FDA cleared system for enumeration of CTC in metastatic breast, metastatic colon and metastatic prostate cancer patients. Finally, the challenges that are addressed in this thesis are described and an outline of the thesis is given.

Chapter 2 describes the CellTracks TDI system, which is an automated image cytometer that features continuous signal acquisition by a TDI camera to minimize overhead time and a homogeneous square illumination area. It measures quantitative fluorescence and morphological features at high sensitivity, high resolution, and is able to relocate events of interest for further investigation. The optical resolution of the system in the scan direction and the direction perpendicular to the scan direction are similar and close to the theoretical maximum resolution. Also, the CellTracks TDI was used to show the heterogeneity of CTC and objects that resemble CTC in patient samples. Figure 1 shows the heterogeneity in tumor cells that have been imaged with the CellTracks TDI system.

In chapter 3, the CellTracks TDI was used to enumerate CTC in blood samples from 68 cancer patients and 9 healthy donors. An automated classification method, using Random Forests, was developed, which eliminates the need for manual review of events. The later is the main cause of 

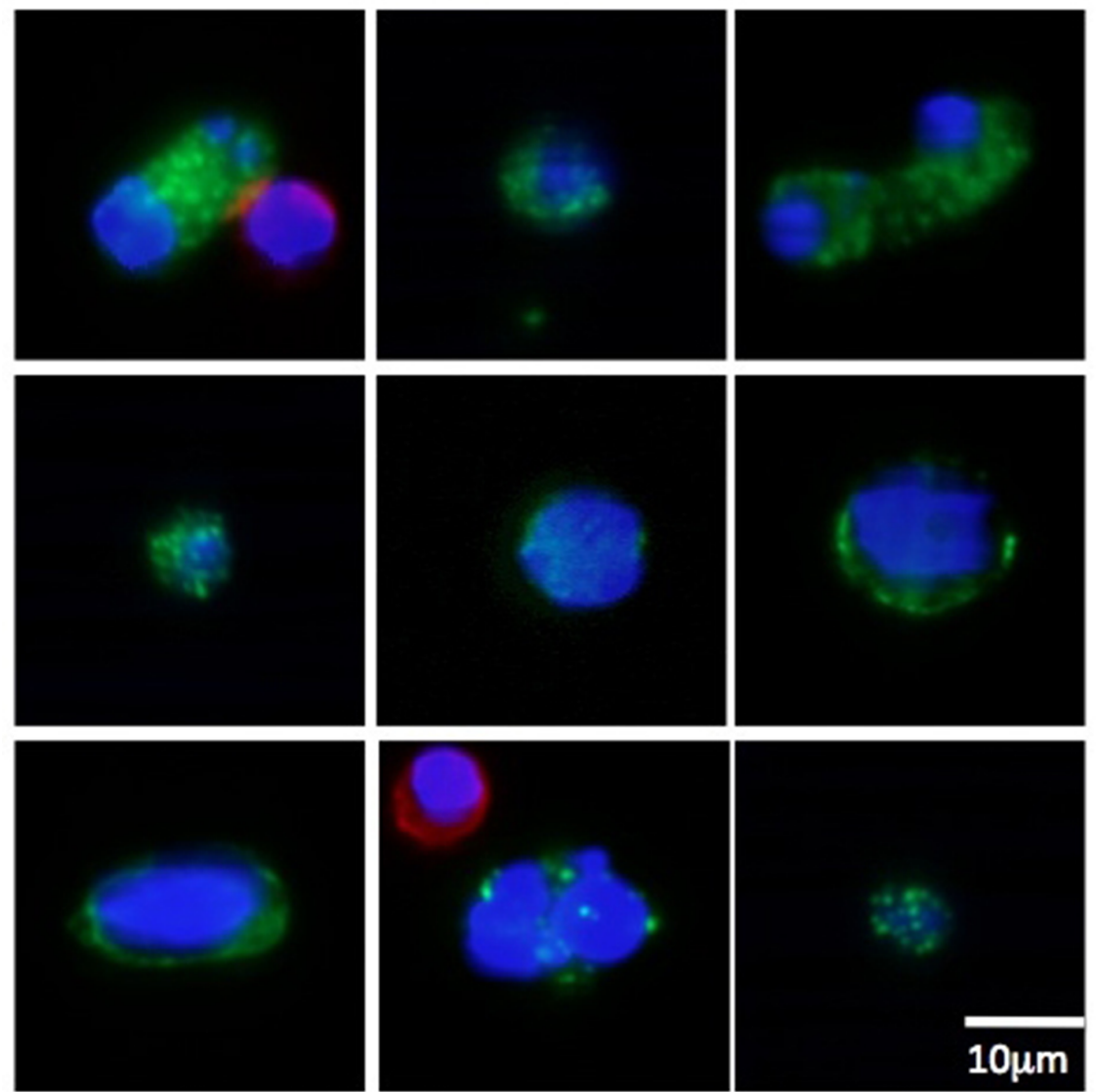

Figure 1 : Tumor cells in blood. Blue: cell nucleus, Red: CD45 (white blood cell), Green: cytokeratin (tumor cell).

inter-laboratory variation in assigning candidate events as CTC. The automated method classifies events as intact CTC, apoptotic CTC, CTC debris, leukocytes or debris, with a high agreement in classification between the automated classifier and 5 expert reviewers. Direct comparison of CTC as imaged in the CellTracks Analyzer II and CellTracks TDI showed improved resolution, improved classification by additional bright-field images and improved CD45-APC detection efficiency by CellTracks TDI. Furthermore, CTC counts by the CellTracks Analyzer II and CellTracks TDI were linear with a high correlation. In conclusion, the automated classification of CTC as intact CTC, apoptotic CTC and CTC debris eliminates the operator error and enables more detailed CTC characterization in cancer patients. Also, CellTracks TDI allows for quantitative assessment of specific treatment targets on CTC. 
Chapter 4 describes the design, development and testing of 4 cell alignment microstructures that mechanically concentrate cells on the analysis surface. Several cleanroom technologies and PDMS micro-molding were used. The microstructures were evaluated for image quality, capture efficiency, alignment efficiency, fluorescence detection efficiency and suitability for implementation in the CellTracks TDI system. SEM pictures of PDMS molds show excellent replication of structures with smooth surfaces and low surface roughness, which enables optimal cell movement. The best structure had a capture efficiency of $>99 \%$, excellent alignment efficiency with 6 channels each being $80 \mu \mathrm{m}$ wide and unobstructed imaging of CTC.

The best microstructure, identified in chapter 4 , for implementation into the CellTracks TDI system is presented in chapter 5. A bright-field autofocus method was developed, which works up to a concentration of $2 \mu \mathrm{g} / \mathrm{ml}$. It uses a spline fit for each of the 6 microstructure channels to enable feed-forward focusing. The linearity of recovery was excellent in buffer samples spiked with SKBR-3 cells between standard CellSearch cartridges and micro-structured cartridges. Also, the microstructures prevent slow sideways movement of cells in samples with low concentrations of free ferrofluid as compared to a standard CellSearch cartridge. With the successful implementation of the microstructures in CellTracks TDI, the imaging times for a CTC sample, using 3 fluorescence- and 1 bright-field channel, were reduced from 38 to 13 minutes, and image analysis times were reduced from 65 to $27 \mathrm{~min}$.

EpCAM-Ferrofluid is added in excess during processing of samples in the AutoPrep to drive the kinetics of binding target cells. Although some free ferrofluid, which did not bind to cells, is removed in the AutoPrep procedure, a significant concentration of $40 \mu \mathrm{g} / \mathrm{ml}$ is present in the final processed sample. In chapter $\mathbf{6}$, we determined the quantitative and qualitative influence of free ferrofluid on bright-field- and fluorescence imaging of beads, T-lymphocytes and CTC. We found that the total fluorescence intensity of beads and cells is reduced by 11-67\%, with the highest reductions in the blue region, in a sample containing $40 \mu \mathrm{g} / \mathrm{ml}$ of free ferrofluid compared to a sample that doesn't contain free ferrofluid. A reduction of the free ferrofluid concentration to below $2 \mu \mathrm{g} / \mathrm{ml}$ is needed to enable optimal detection of fluorescence signals from cells and to permit the identification of the cell surface by using bright-field images.

Chapter 7 discusses the automated method that was developed to remove free ferrofluid from samples after immunomagnetic enrichment of CTC. An automated ferrofluid removal system (AFRS) was designed to remove $>95 \%$ of the free ferrofluid in an AutoPrep sample, with a recovery of $>90 \%$ of CTC. The AFRS rotates an AutoPrep sample tube around its longitudinal axis and aspirates a fraction of the sample in the tube during rotation, thereby removing mostly free ferrofluid. A Matlab simulation was constructed to determine the required minimum rotation time and the results of the simulation were compared to experiments with samples that 
contained SKBR-3 cells spiked in buffer. The concentration of free ferrofluid was determined from absorbance measurements at $420 \mathrm{~nm}$. The AFRS was tested with whole blood samples spiked with SKBR-3- and PC3-9 cells and with samples from prostate cancer patients. Both the removal efficiency of free ferrofluid and the recovery of CTC were above the design specification for spiked tumor cells in buffer. The removal efficiency of free ferrofluid was close to the design specification for samples containing spiked tumor cells in whole blood as well as samples from prostate cancer patients. However, the recovery of CTC for those samples was significantly lower than the design specification at $77 \%$ and $56 \%$ for the spiked whole blood samples and the prostate cancer samples respectively.

\section{OUTLOOK}

The automated image cytometer, CellTracks TDI, described in this thesis improves the imaging and characterization of CTC in blood samples that are immunomagnetically enriched and fluorescently labeled by the CellTracks AutoPrep system.

Further improvements to the detection efficiency of CTC can be made by identification of all nucleated cells. Although the efficiency of CD45-APC fluorescence detection has been improved, the origin of not all nucleated cells can be accounted for. Investigation of other lineage markers may help to identify the unidentified cells. If these cells are not tumor cells, the marker used to identify them can be labeled with APC and combined with CD45-APC to further reduce false-positive CTC scoring. Likewise, markers can be discovered that identify CTC that are not identified by the current cocktail of cytokeratin antibodies. These markers can then be labeled with $\mathrm{PE}$ and combined with CK-PE to improve the sensitivity of CTC detection. Such an approach may also aid in the detection and characterization of cells that have undergone an epithelial-mesenchymal transition (EMT), resulting in stem cell like properties. These cells may be more effective in tissue invasion and formation of metastases as compared to epithelial cells that have not undergone this transition.

To use CTC as a "liquid biopsy" for personalized medicine, the number of treatment targets that can be identified on CTC will need to be increased. Presence and status of the ER, PR, HER1, HER2, IgF1R and VEGFR receptors, and markers indicating resistance to chemotherapy, would dramatically increase the ability to guide treatment of the individual metastatic breast cancer patient. To identify multiple treatment targets simultaneously, the number of fluorescence channels in the CellTracks TDI system has to be increased, in combination with the use of additional fluorophores, using appropriate cross-talk correction. Another possibility is to re-analyze sample cartridges with the CellTracks TDI that have been previously analyzed by the CellTracks Analyzer II. The sample cartridges can be re-stained with 
for example FISH probes, provided the position of the CTC has not altered significantly, to determine amplification, translocation or deletion of certain genes.

The sensitivity of the detection of antigens by the CellTracks TDI system can be improved by removal of free ferrofluid from AutoPrep processed samples. Although the free ferrofluid could be effectively removed by the AFRS, it was accompanied by a loss of CTC, which is not acceptable. Further improvement is needed and can be obtained by for example increasing the rotation time for each withdrawal step or by using a slightly different method, in which a magnetic rod is used, instead of aspiration or by means of filtration, making use of the smaller size of ferrofluids as compared to CTC.

In conclusion, the microscopic characterization of CTC has been greatly improved by the results presented in this thesis. However, to serve as a liquid biopsy for the majority of patients, CTC will need to be detected in these patients and significant efforts to increase the percentage of patients in which CTC are detected are needed. Increasing the volume of blood analyzed by development of in-vivo cytometry technology or CTC apheresis, in which the blood void of tumor cells is returned to the patient, may address this issue. The combination of exhaustive characterization of detected CTC and the use of larger blood volumes will eventually lead to improved personal treatment, bringing cancer one step closer to being a chronic disease. 



\section{SAMENVATTING}

\section{Conclusies}

Het tellen en karakteriseren van circulerende tumor cellen (CTC), om de behandeling van patiënten met kanker te verbeteren, is in opkomst voor de ziektebeheersing van patiënten met gemetastaseerde kankers. De detectie van CTC wordt belemmerd door de zeer lage frequentie waarmee deze cellen in het bloed van patiënten voorkomen. Het CellSearch systeem is het eerste systeem dat gevalideerd is voor het opsporen van CTC in de kliniek en dit proefschrift beschrijft de resultaten van onderzoek dat gedaan is om dit systeem verder te verbeteren.

Hoofdstuk 1 geeft een introductie van kanker in het algemeen en van CTC in het bijzonder. CTC zijn gecorreleerd aan incident vrije- en algemene overlevingskansen in meerdere soorten gemetastaseerde kanker en CTC kunnen gebruikt worden om patiënten in te delen in lage en hoge risico groepen. Het aantal CTC in bloed hebben een betere prognostische waarde dan radiologische methoden, die op dit moment alom worden gebruikt in de kliniek, in termen van bijvoorbeeld correlatie met algemene overlevingskansen. Ook worden in dit hoofdstuk een aantal nieuwe CTC detectie methoden beschreven en wordt er een introductie gegeven van het CellSearch systeem, dat op dit moment het enige FDA goedgekeurde systeem is voor de enumeratie van CTC in gemetastaseerd borst, darm en prostaat kanker. Als laatste worden de uitdagingen beschreven die in dit proefschrift worden geadresseerd, gevolgd door een overzicht van dit proefschrift.

Hoofdstuk 2 beschrijft het CellTracks TDI systeem, een geautomatiseerde afbeelding cytometer met een homogeen vierkant belichtingsprofiel en een continue signaalacquisitie door een TDI camera om de overhead tijd te minimaliseren. Het CellTracks TDI systeem meet kwantitatieve fluorescentie en morfologische kenmerken en kan interessante objecten terugvinden voor verdere analyse. De optische resolutie van het systeem in de scan richting en de richting daar loodrecht op zijn vergelijkbaar en liggen dicht bij de theoretische maximum resolutie. Tevens is, met behulp van het CellTracks TDI systeem, de heterogeniteit van CTC en objecten die lijken op CTC in patiënten samples aangetoond. Figuur 1 laat de heterogeniteit in tumor cellen zien die zijn afgebeeld met het CellTracks TDI systeem. 

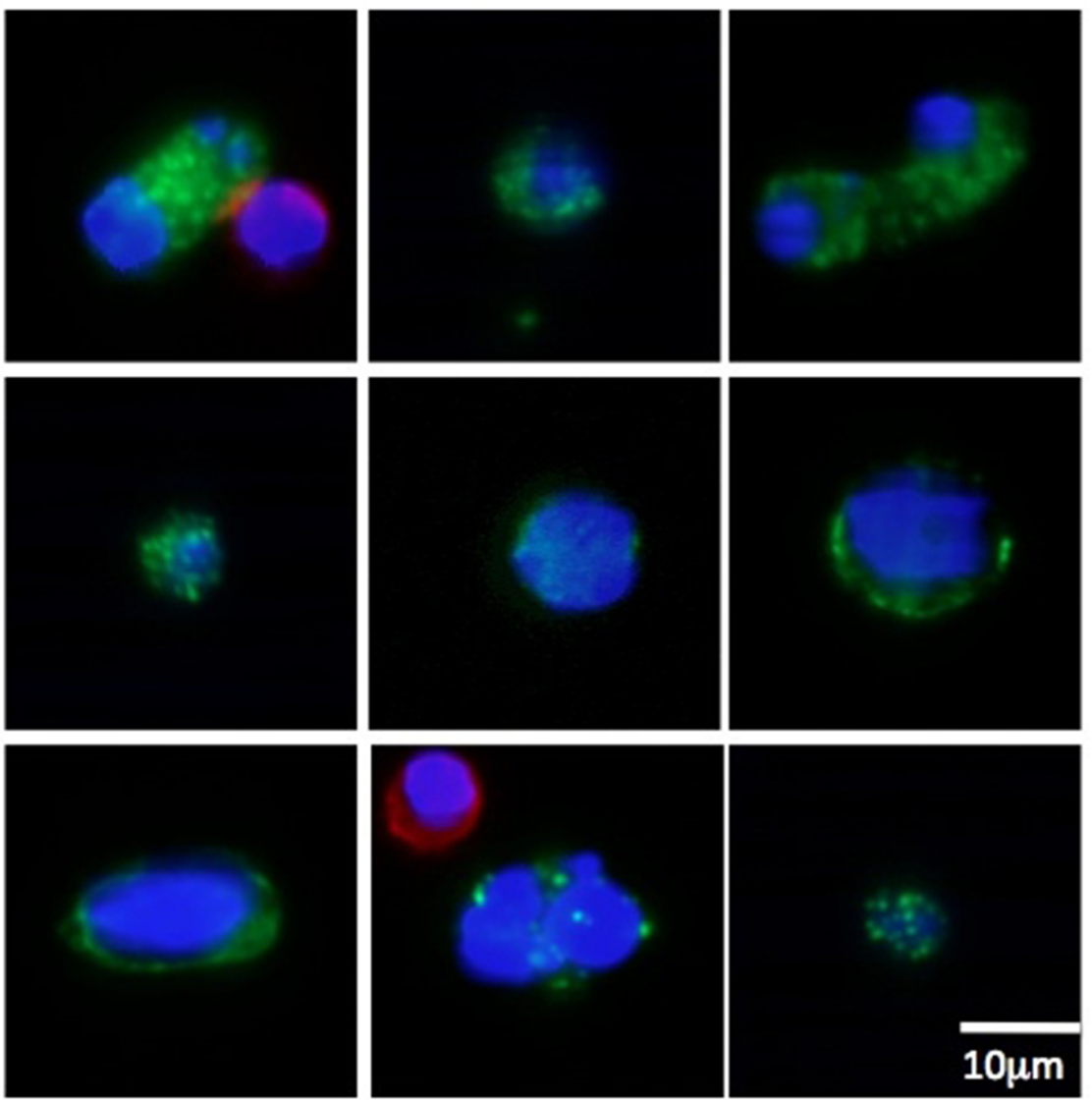

FiguUR 1 : Tumor cellen in bloed. Blauw: cel kern, Rood: CD45 (witte bloed cel), Groen: cytokeratine (tumor cel).

In hoofdstuk 3 is het CellTracks TDI systeem gebruikt om CTC te tellen in bloed samples van 68 patiënten met kanker en 9 gezonde donoren. Een geautomatiseerde classificatie methode, gebaseerd op "Random Forests", werd ontwikkeld die handmatige controle van objecten overbodig maakt. Handmatige classificatie is de hoofdoorzaak van variatie tussen laboratoria bij het aanmerken van objecten als CTC. De geautomatiseerde methode classificeert objecten als intacte CTC, apoptotische CTC, CTC fragmenten, leukocyten of andere (cel) fragmenten, met een hoge overeenstemming met 5 deskundige beoordelaars. Een directe vergelijking van CTC gemeten met de CellTracks Analyzer II en CellTracks TDI laat een verbeterde resolutie, verbeterde classificatie (d.m.v. helder-veld beelden) en een verbeterde CD45-APC detectie efficiëntie zien bij gebruik van de CellTracks TDI. Tevens was het aantal gedetecteerde CTC lineair met een hoge correlatie 
tussen de CellTracks Analyzer II en de CellTracks TDI. Geautomatiseerde classificatie van CTC als intacte CTC, apoptotische CTC en CTC resten voorkomt menselijke fouten bij het classificeren van CTC en het maakt een gedetailleerdere karakterisering van CTC in patiënten met kanker mogelijk. Ook is het met het CellTracks TDI systeem mogelijk om behandeldoelwitten op CTC op een kwantitatieve manier te beoordelen.

Hoofdstuk 4 beschrijft het ontwerp, de ontwikkeling en het testen van 4 microstructuren die cellen mechanisch op een analyse oppervlak concentreren. Verschillende cleanroom technologieën en PDMS afdrukken werden hierbij gebruikt. De microstructuren werden beoordeeld op beeldkwaliteit, afvangrendement, uitlijningsefficiëntie, efficiëntie van fluorescentie detectie en geschiktheid voor gebruik in het CellTracks TDI systeem. SEM foto's van PDMS microstructuren vertonen uitstekende replicatie van structuren met een glad oppervlak en een lage ruwheid van het oppervlak hetgeen een optimale beweging van de cellen langs het oppervlak mogelijk maakt. De beste structuur had een afvangrendement van $>99 \%$, een uitstekende uitlijningsefficiëntie met 6 kanalen die elk $80 \mu \mathrm{m}$ breed zijn en de mogelijkheid tot onbelemmerde beeldvorming van CTC.

De implementatie van de beste microstructuur in het CellTracks TDI systeem, zoals bepaald in hoofdstuk 4, wordt beschreven in hoofdstuk 5. Een geautomatiseerde methode voor het focusseren werd hiervoor ontwikkeld, die werkt tot een concentratie van vrije ferrofluid van $2 \mu \mathrm{g} / \mathrm{ml}$. Deze methode gebruikt een spline fit voor het afzonderlijk feed-forward focusseren op elk van de 6 kanalen. Het percentage cellen dat terug kon worden gevonden in buffer samples met SKBR-3 cellen was lineair tussen standaard CellSearch analyse kamers en analyse kamers met microstructuren. Daarnaast voorkomen de analyse kamers met microstructuren de zijwaartse beweging van cellen in samples met een lage vrije ferrofluid concentratie in vergelijking met standaard CellSearch analyse kamers. Door de succesvolle implementatie van de microstructuren in het CellTracks TDI systeem is de afbeeldingtijd van een CTC sample in 3 fluorescentie kanalen en 1 helderveld kanaal verlaagd van 38 naar 13 minuten en de beeldverwerkingstijd teruggebracht van 65 naar 27 minuten.

EpCAM-Ferrofluid wordt in een overmaat toegevoegd tijdens het verwerken van samples in het AutoPrep systeem om zoveel mogelijk ferrofluid deeltjes te laten binden aan CTC. Hoewel een gedeelte van de ferrofluid dat niet gebonden is aan cellen wordt verwijderd in de AutoPrep, blijft er een significante concentratie vrije ferrofluid over van $40 \mu \mathrm{g} / \mathrm{ml}$ in de uiteindelijke samples zoals verwerkt door de AutoPrep. In hoofdstuk 6 wordt de kwantitatieve- en kwalitatieve invloed van vrije ferrofluid op de helder-veld en fluorescentie afbeeldingen van bolletjes, T-lymphocyten en CTC bepaald. We hebben bepaald dat in een sample met $40 \mu \mathrm{g} / \mathrm{ml}$ aan vrije ferrofluid vergeleken met een sample zonder vrije ferrofluid de totale fluorescentie intensiteit van bolletjes en cellen wordt verlaagd met 11 tot $67 \%$, met de hoogste verliezen in het blauwe gedeelte van het spectrum. Een 
verlaging van de concentratie van de vrije ferrofluid tot beneden $2 \mu \mathrm{g} / \mathrm{ml}$ is noodzakelijk om optimale detectie van fluorescentie signalen mogelijk te maken en om de identificatie van de celwand mogelijk te maken met behulp van helder-veld beelden.

Hoofdstuk 7 bespreekt de geautomatiseerde methode die werd ontwikkeld om vrije ferrofluid te verwijderen uit samples nadat deze immunomagnetisch zijn verrijkt voor CTC detectie. Een geautomatiseerd ferrofluid verwijdering systeem (GFVS) werd ontwikkeld om meer dan $95 \%$ van de vrije ferrofluid te verwijderen en meer dan $90 \%$ van de CTC te behouden in een sample dat verrijkt is in de AutoPrep. Het GFVS roteert een sample buis uit de AutoPrep om zijn lengte-as en aspireert een gedeelte van de vloeistof in de buis gedurende rotatie van de buis, waarmee hoofdzakelijk vrije ferrofluid wordt verwijderd.

Een simulatie in Matlab werd ontwikkeld om de minimale rotatie tijd te bepalen en de resultaten van de simulatie werden vergeleken met resultaten van experimenten met samples die SKBR-3 cellen in buffer bevatten. De concentratie vrije ferrofluid in samples werd bepaald door middel van absorptie metingen bij $420 \mathrm{~nm}$. Het GFVS is getest met bloed samples verrijkt met SKBR-3 en PC3-9 cellen en met bloed samples van patiënten met prostaat kanker. Zowel de efficiëntie van vrije ferrofluid verwijdering als de terugwinning van CTC zijn boven de ontwerp specificatie voor buffer samples die zijn verrijkt met tumor cellen. Terwijl voor bloed samples die verrijkt zijn met tumor cellen en voor bloed samples van patiënten met prostaat kanker de verwijdering efficiëntie van vrije ferrofluid dicht bij de ontwerp specificatie lag. De terugwinning van CTC voor deze samples was echter significant lager dan de ontwerp specificatie met respectievelijk $77 \%$ voor bloed samples verrijkt met tumor cellen en $56 \%$ voor samples van patiënten met prostaat kanker.

\section{VOORUITZICHTEN}

De geautomatiseerde afbeelding cytometer, CellTracks TDI, die in dit proefschrift wordt beschreven verbeterd het afbeelden en het karakteriseren van CTC in bloed samples die immunomagnetisch verrijkt en fluorescent gelabeld zijn door het CellTracks AutoPrep systeem.

De efficiëntie van CTC detectie kan verbeterd worden door de identificatie van alle cellen die een kern bevatten. Ondanks de verbeterde detectie efficiëntie van CD45-APC, kunnen nog niet alle cellen die een kern bevatten exact worden gekarakteriseerd. Onderzoek met behulp van andere afstammingsmarkers kan meehelpen in de herkenning van cellen die tot op heden nog niet geïdentificeerd kunnen worden. Als de onderzochte cellen geen tumor cellen zijn kan de betreffende marker gelabeld worden met APC en toegevoegd worden aan CD45-APC om de hoeveelheid vals positieve CTC te verminderen. Ook kunnen markers ontdekt worden die CTC herkennen 
die op dit moment niet geïdentificeerd worden door de huidige cocktail van cytokeratine antilichamen. Deze markers kunnen dan gelabeld worden met $\mathrm{PE}$ en toegevoegd worden aan CK-PE om de gevoeligheid van CTC detectie te verhogen. Een dergelijke benadering kan ook helpen om cellen die een epitheel-mesenchymale transitie (EMT) hebben ondergaan, wat resulteert in eigenschappen gelijk aan stamcellen, te detecteren en te karakteriseren. Dit type cellen is mogelijk effectiever in het binnendringen van weefsel en het vormen van uitzaaiingen in vergelijking met epitheel cellen die deze transitie niet hebben ondergaan.

Om CTC te kunnen gebruiken voor een "vloeibare biopsie" voor een gepersonaliseerde behandeling moet het aantal behandel doelwitten dat geïdentificeerd kan worden op een CTC verhoogd worden. Inzage in de aanwezigheid en de staat van de ER, PR, HER1, HER2, IgF1R en VEGFR receptoren en markers die resistentie tegen chemotherapie aangeven is belangrijk om de persoonlijke behandeling van patiënten met borstkanker te kunnen sturen. Om meerdere behandelingsdoelen tegelijk te kunnen identificeren, moet het aantal fluorescentie kanalen in het CellTracks TDI systeem verhoogd worden in combinatie met het gebruik van aanvullende fluorescente markers aangevuld met correcties voor spectrale overlap tussen fluorescentie kanalen. Een andere verbetermogelijkheid is om sample kamers opnieuw te analyseren met het CellTracks TDI systeem die eerder geanalyseerd zijn met de CellTracks Analyzer II. De sample kamers kunnen dan bijvoorbeeld opnieuw gelabeld worden met behulp van fluorescente in situ hybridisatie (FISH), vooropgesteld dat de positie van de CTC niet significant veranderd is, om versterking, verplaatsing of verwijdering van bepaalde genen te bepalen.

De gevoeligheid waarmee antigenen gedetecteerd worden in het CellTracks TDI systeem kan verbeterd worden door de verwijdering van vrije ferrofluid uit samples die verwerkt zijn door de AutoPrep. Hoewel de vrije ferrofluid effectief verwijderd kon worden door het GFVS, werd dit vergezeld van een verlies van CTC, wat niet acceptabel is. Verdere verbetering is noodzakelijk en kan bijvoorbeeld verkregen worden door de rotatie tijd tussen elke verwijderstap te verhogen of door een enigszins andere methode te gebruiken waarbij een magnetische staaf gebruikt wordt, in plaats van aspiratie, of door middel van filtratie, waarbij gebruik wordt gemaakt van de kleinere grootte van ferrofluid in vergelijking tot CTC.

Concluderend kunnen we stellen dat de microscopische karakterisering van CTC sterk verbeterd is door wat beschreven staat in dit proefschrift. Om echter te kunnen dienen als een vloeibare biopsie voor de meerderheid van patiënten, moeten CTC gedetecteerd kunnen worden in deze patiënten en daarvoor zijn aanzienlijke inspanningen nodig om het percentage patiënten waarbij CTC worden gevonden te verhogen. Het vergroten van het bloedvolume dat geanalyseerd wordt door ontwikkeling van in-vivo cytometrie technologieën of door CTC aferese, waarbij bloed dat ontdaan is van tumor cellen wordt teruggegeven aan de patiënt, kunnen deze kwestie 
adresseren. De combinatie van volledige karakterisering van gedetecteerde CTC en het gebruik van grotere bloedvolumes zal uiteindelijk lijden tot een verbeterde persoonlijke behandeling voor de patiënt, waardoor kanker een stap dichter bij een chronische ziekte komt. 


\section{LIST OF PUBLICATIONS}

\section{PATENTS}

Magnetic separation apparatus and methods. Inventors: Tycho M Scholtens, Leon WMM Terstappen, Arjan GJ Tibbe. United States Patent No: 7,666,308 B2, February 23, 2010.

\section{JOURNAL ARTICLES}

Scholtens, T.M., Schreuder, F., Ligthart, S.T., Swennenhuis, J.F., Tibbe, A.G.J., Greve, J. \& Terstappen, L.W.M.M. "CellTracks TDI: an Image Cytometer for Cell Characterization". Cytometry Part A 79(3): 203-213, 2011.

Scholtens, T.M., Schreuder, F., Ligthart, S.T., Swennenhuis, J.F., Greve J. \& Terstappen, L.W.M.M. Automated Identification of Circulating Tumor Cells by Image Cytometry. Cytometry Part A 81(2): 138-148, 2012.

\section{Conference COntributions (ORAL)}

Li, X., Ymeti, A., Droog, E.J., Schreuder, F., Scholtens, T.M., Greve, J., Tibbe, A.G.J. \& Terstappen, L.W.M.M. "EasyCount, an Affordable and Simple Cell Enumeration System". ALW / FOM / VvBBMT Annual Dutch Meeting on Molecular and Cellular Biophysics 2004, September 27 September 28, Lunteren, the Netherlands.

Scholtens, T.M., Schreuder, F., Greve, J., Tibbe, A.G.J. \& Terstappen, L.W.M.M. "Cell aligning microstructures for image cytometry". Focus on Microscopy 2007, April 10 - April 13, Valencia, Spain.

\section{Conference COntributions (Poster)}

Scholtens, T.M., Schreuder, F., Greve, J., Tibbe, A.G.J. \& Terstappen, L.W.M.M. "A novel design for aligning (rate) cells using PDMS micromolding". ALW / FOM / VvBBMT Annual Dutch Meeting on Molecular and Cellular Biophysics 2004, September 27 - September 28, Lunteren, the Netherlands. 
Ymeti, A., Scholtens, T.M., Schreuder, F., Greve, J., Tibbe, A.G.J. \& Terstappen, L.W.M.M. "An affordable and accurate enumeration system for HIV-staging". ALW / FOM / VvBBMT Annual Dutch Meeting on Molecular and Cellular Biophysics 2004, September 27 - September 28, Lunteren, the Netherlands.

Scholtens, T.M., Schreuder, F., Greve, J., Tibbe, A.G.J. \& Terstappen, L.W.M.M. "Aligning and analyzing (rare) cells using PDMS micro-molding". ALW / FOM / VvBBMT Annual Dutch Meeting on Molecular and Cellular Biophysics 2005, October 9 - October 10, Lunteren, the Netherlands.

Schreuder, F., Scholtens, T.M., Greve, J., Tibbe, A.G.J. \& Terstappen, L.W.M.M. "Improved imaging pinpoints circulating tumour cells". ALW / FOM / VvBBMT Annual Dutch Meeting on Molecular and Cellular Biophysics 2005, October 9 - October 10, Lunteren, the Netherlands.

Scholtens, T.M., Schreuder, F., \& Greve, J. "Cell orienting microstructures for image cytometry". XXIII Congress of the International Society for Analytical Cytometry 2006, May 20 - May 24, Quebec City, Canada.

Schreuder, F., Scholtens, T.M., Ligthart, S.T., \& Greve, J. "A Fast imaging cytometer based on time delay integration". XXIII Congress of the International Society for Analytical Cytometry 2006, May 20 - May 24, Quebec City, Canada.

Scholtens, T.M., Schreuder, F., Greve, J., Tibbe, A.G.J., \& Terstappen, L.W.M.M. "Cell orienting microstructures for image cytometry". ALW / FOM / VvBBMT Annual Dutch Meeting on Molecular and Cellular Biophysics 2006, October 9 - October 10, Lunteren, the Netherlands.

Scholtens, T.M., Schreuder, F., Greve, J., Tibbe, A.G.J. \& Terstappen, L.W.M.M. "Improved imaging of immunomagnetically enriched rare cells". XXIV Congress of the International Society for Advancement of Cytometry 2008, May 17 - May 21, Budapest, Hungary.

Schreuder, F., Scholtens, T.M., Ligthart, S.T., Tibbe, A.G.J., Swennenhuis, J.F., Greve, J. \& Terstappen, L.W.M.M. "Characterization of circulating tumor cells by image cytometry". XXIV Congress of the International Society for Advancement of Cytometry 2008, May 17 - May 21, Budapest, Hungary.

Scholtens, T.M., Schreuder, F., Ligthart, S.T., Swennenhuis, J.F., Tibbe A.G.J., Greve, J. \& Terstappen, L.W.M.M. "CellTracks TDI: An image cytometer for cell characterization". Lorentz meeting on circulating tumor cell isolation and diagnostics 2011, February 7 - February 11, Leiden, the Netherlands.

\section{ARTICLE IN PROCEEDINGS}

Ymeti, A., Li, X., Scholtens, T.M., Schreuder, F., Greve, J., Tibbe, A.G.J. \& Terstappen, L.W.M.M. "Development of an affordable cell enumaration system for HIV staging". Proceedings of the Dutch Annual Conference 
on BioMedical Engineering 2004, pp 180-181, Grafisch Centrum Twente, Enschede, the Netherlands. 



\section{DANKWOORD}

Zo daar zit ik dan, op mijn balkon met stralend weer en een licht briesje. Goed moment om de laatste paar jaar eens de revue te laten passeren. Na iets meer dan 4 jaar is de missie die mijn promotie is geworden dan eindelijk volbracht! Na mijn afstuderen werd ik door Jan en Leon gevraagd om door te gaan in het onderzoek door het doen van een promotie. Dit aanbod heb ik aanvaard omdat het ontwikkelen van technieken aan het voorfront van de wetenschap mij aansprak, en nog steeds doet, zeker met de prachtige toepassing die wij ermee hadden, hebben en in de toekomst nog veel meer zullen hebben. Ik ben erg trots dat ik hier mijn steentje aan heb kunnen bijdragen! Dit alles heb ik natuurlijk niet alleen gedaan, maar met de hulp van velen. Daarmee komen we dan direct aan bij de twee personen die ik het eerst wil bedanken.

Jan, van harte bedankt voor de ondersteuning en inspiratie in de eerste helft van mijn promotie. Jij wist altijd de fysische kant van de zaak te benadrukken en dit heb ik altijd gewaardeerd als natuurkundige. Zeker ook omdat het mij alert hield, soms zag jij dingen op een manier die ik in eerste instantie niet zag.

Leon, van harte bedankt voor de excellente begeleiding in de laatste jaren, al ging het soms niet even gemakkelijk. Ook bedankt voor de unieke gave om mij altijd weer te inspireren bij het bespreken van nieuwe resultaten en misschien nog wel meer bij het bespreken van problemen waar ik niet direct uit kwam.

Vervolgens wil ik graag Erik bedanken voor de excellente samenwerking gedurende vele jaren en talloze uren samen in het microscopie lab, knutselend aan de opstelling. Ook nog bedankt voor de gezellige conferenties, ook al moest ik je dan om 3 uur 's nachts weer in de kamer laten. Lilly en Aurel: thank you for you support and the good discussions and experiences we had in the Cytometry group.

Gedurende de jaren heb ik een groot aantal (bloed)samples verwerkt en laten verwerken. Hiervoor heb ik vaak de hulp ingeroepen van de mensen bij Immunicon/Veridex Enschede. Arjan, Alexander, Bjorn en Rianne: bedankt voor het verwerken van samples (ook als het eigenlijk niet kon), de koekjes bij de koffie en het leren welke cel volgens de CellSearch definitie nu wel en niet een CTC is. Al blijft dat laatste natuurlijk operator-dependent! Verder wil ik graag Yvonne, Kirsten en Wilma bedanken voor het kweken 
van cellijnen en voor het afnemen van talloze buizen bloed, al ging dat bij het ene slachtoffer soms makkelijker dan bij het andere. For the patient samples that I used in the last chapter I would like to express my gratitude to Johann and Gert from Royal Marsden. Verder wil ik graag Dr. M. de Groot van het MST ziekenhuis te Enschede bedanken voor de patiënt samples die gebruikt zijn in hoofdstuk 3 .

Syl en Ingrid: bedankt voor de bijstand in allerlei zaken. Frans: bedankt voor je altijd nuchtere kijk en het repareren van mijn fiets. Sjoerd, Roy, Erwin, Liesbeth, Bart, Johan, Aufried, Kees, Robert, Frank, Guus, Joost, Niels, Kiki, Markus and Elia, and all the room- and groupmates i had over the years in the Hogekamp, Zuidhorst and Carre buildings: Thank you for discussions on science and, more important, non-science stuff whether it be in the office, coffee corner or over some beers! Verder wil ik ook graag Tjeerd en Barbara bedanken voor advies in tijden waarin de zon even achter de wolken verdwenen leek te zijn, dank!

Naast uitlijnen van de opstelling, samples meten en schrijven van lappen tekst moest er natuurlijk ook aan ontspanning gedaan worden. Hiervoor wil ik allereerst graag de lui van de pokerclub bedanken. De combinatie van poker, bier, slap ouwehoeren en ook diepzinnige discussies bevalt mij nog steeds erg goed. Daan, Willem, Hanneke, Joris, Sandra, Alfred, Vanessa, Steven, Danielle, Azzeddine, Joep en Anoek: hartelijk dank voor de vakanties, de vele feestjes en de altijd legendarische Koninginnedagen.

Christian, bedankt voor alle kilometers die we samen gemaakt hebben op de racefiets, je onmisbare hulp in het lab en daarbuiten, je organisatietalent en dat je mijn paranimf wilt zijn! Wouter en Erica, bedankt voor de steun en het vertrouwen en Wouter bedankt dat je mijn andere paranimf wilt zijn!

Pap en mam, heel hartelijk dank voor jullie onvoorwaardelijke steun in makkelijke en zeker ook moeilijke tijden. Dit waardeer ik nog steeds erg veel en zonder jullie steun was het zeker niet gelukt, bedankt!

Enschede, 12 augustus 2012 


\section{ABOUT THE AUTHOR}

Tycho Marinus Scholtens was born on the $26^{\text {th }}$ of September 1978 in Oldenzaal, the Netherlands. He graduated from high school at the Twents Carmel Lyceum in Oldenzaal in 1996. In that same year, he started studying Applied Physics at the University of Twente in Enschede. In 2001, he did his internship at Cytopeia Inc., Seattle, U.S.A. He graduated in 2004 on the subject "Technical aspects of aligning and analyzing rare cells in blood" in the BioPhysical Engineering group of Prof. Dr. Jan Greve. He started his Ph.D. research in the same group, focusing on the improved and automated imaging of circulating tumor cells, and finished his research in the Medical Cell BioPhysics group of Prof. Dr. Leon Terstappen. The results of this research are described in this thesis. 